\title{
The modular synthesis of chiral NHC precursors and their silver and gold complexes
}

Zita Szabo, ${ }^{\text {a }}$ Matyas Timari, ${ }^{\text {a }}$ Rudolf Kassai, ${ }^{\text {ał }}$ Bianka Szokol, ${ }^{a} \rrbracket$ Attila Benyei, ${ }^{b}$ Tamas Gati, ${ }^{a}$ Attila Paczal ${ }^{a *}$ and Andras Kotschy ${ }^{a *}$

aServier Research Institute of Medicinal Chemistry, Zahony u 7., H-1031 Budapest, Hungary, and bepartment of Physical Chemistry, University of Debrecen, Egyetem ter $1, \mathrm{H}$ 4032 Debrecen, Hungary

\section{Contents}

Experimental details of X-ray diffraction measurement .$\$ 2$

$1 \mathrm{H}$ and $13 \mathrm{C}$ NMR spectra of the prepared compounds .S4

\footnotetext{
‡ Present addresses: Rudolf Kassai: Elek utca 22, H-1113 Budapest, Hungary. Bianka Szokol: Department of Organic Chemistry and Technology, Budapest University of Technology and Economics, Szent Gellert ter 4, H1111 Budapest, Hungary
} 
Experimental details of $\mathrm{X}$-ray diffraction measurement

\begin{tabular}{|c|c|c|c|c|}
\hline & $(22 \mathrm{i})$ & (23a) & $(23 c)$ & (23d) \\
\hline \multicolumn{5}{|l|}{ Crystal data } \\
\hline Chemical formula & $\mathrm{C}_{25} \mathrm{H}_{34} \mathrm{AuClN}_{2}$ & $\mathrm{C}_{31} \mathrm{H}_{48} \mathrm{AuClN}_{2} \cdot \mathrm{CH}_{2} \mathrm{Cl}_{2}$ & $\mathrm{C}_{33} \mathrm{H}_{42} \mathrm{AuClN}_{2}$ & $\mathrm{C}_{33} \mathrm{H}_{42} \mathrm{AuClN}_{2}$ \\
\hline$M_{\mathrm{r}}$ & 594.96 & 766.05 & 699.10 & 699.10 \\
\hline Crystal system, space group & $\begin{array}{l}\text { Orthorhombic, } \\
P 2_{1} 2_{1} 2_{1}\end{array}$ & Orthorhombic, $P 2_{1} 2_{1} 2_{1}$ & $\begin{array}{l}\text { Orthorhombic, } \\
P 2{ }_{2} 2_{1} 2\end{array}$ & Monoclinic, $P 2_{1}$ \\
\hline Temperature (K) & 296 & 295 & 297 & 297 \\
\hline$a, b, c(\AA)$ & $\begin{array}{l}7.9933(8), \\
15.6142(13), \\
19.7024(19)\end{array}$ & $\begin{array}{l}12.5577(4), \\
15.8009(4), \\
17.3349(6)\end{array}$ & $\begin{array}{l}15.5664(5), \\
18.6886(7) \\
10.8277(5)\end{array}$ & $\begin{array}{l}10.5394(6), 9.3715 \\
(5), 16.1275(8)\end{array}$ \\
\hline$\alpha, \beta, \gamma\left(^{\circ}\right)$ & $90,90,90$ & $90,90,90$ & $90,90,90$ & $90,101.192(2), 90$ \\
\hline$V\left(\AA^{3}\right)$ & $2459.0(4)$ & $3439.64(18)$ & $3149.9(2)$ & $1562.62(14)$ \\
\hline$Z$ & 4 & 4 & 4 & 2 \\
\hline Radiation type & Mo $K \alpha$ & Mo $K \alpha$ & Mo $K \alpha$ & Mo $K \alpha$ \\
\hline$\mu\left(\mathrm{mm}^{-1}\right)$ & 6.10 & 4.53 & 4.78 & 4.82 \\
\hline Crystal size $(\mathrm{mm})$ & $\begin{array}{l}0.28 \times 0.12 \times \\
0.05\end{array}$ & $0.51 \times 0.25 \times 0.12$ & $\begin{array}{l}0.49 \times 0.20 \times \\
0.10\end{array}$ & $0.23 \times 0.07 \times 0.07$ \\
\hline \multicolumn{5}{|l|}{ Data collection } \\
\hline Diffractometer & \multicolumn{4}{|c|}{ Bruker D8 VENTURE } \\
\hline Absorption correction & \multicolumn{4}{|c|}{$\begin{array}{l}\text { Multi-scan SADABS2016/2 - Bruker AXS area detector scaling and absorption } \\
\text { correction }\end{array}$} \\
\hline$T_{\min }, T_{\max }$ & $0.51,0.75$ & $0.36,0.62$ & $0.42,0.66$ & $0.60,0.74$ \\
\hline $\begin{array}{l}\text { No. of measured, independent } \\
\text { and observed }[I>2 \sigma(I)] \\
\text { reflections }\end{array}$ & $\begin{array}{l}21577,4825 \\
4451\end{array}$ & $18522,6947,5883$ & $\begin{array}{l}24479,9522, \\
6098\end{array}$ & $23396,6376,4927$ \\
\hline$R_{\text {int }}$ & 0.039 & 0.032 & 0.059 & 0.083 \\
\hline$(\sin \theta / \lambda)_{\max }\left(\AA^{-1}\right)$ & 0.617 & 0.625 & 0.714 & 0.626 \\
\hline \multicolumn{5}{|l|}{ Refinement } \\
\hline$R\left[F^{2}>2 \sigma\left(F^{2}\right)\right], w R\left(F^{2}\right), S$ & $\begin{array}{l}0.035,0.115 \\
1.31\end{array}$ & $0.038,0.110,1.13$ & $\begin{array}{l}0.049,0.147 \\
1.00\end{array}$ & $0.041,0.077,0.98$ \\
\hline No. of reflections & 4825 & 6947 & 9522 & 6376 \\
\hline No. of parameters & 270 & 352 & 343 & 342 \\
\hline No. of restraints & 0 & 0 & 0 & 1 \\
\hline H-atom treatment & \multicolumn{4}{|c|}{ H-atom parameters constrained } \\
\hline$\left.\Delta\rangle_{\max }, \Delta\right\rangle_{\min }\left(\mathrm{e} \AA^{-3}\right)$ & $2.49,-1.47$ & $1.71,-1.13$ & $2.18,-1.27$ & $0.64,-0.83$ \\
\hline Absolute structure & $\begin{array}{c}1797 \\
\text { quotients [(I+) }\end{array}$ & $\begin{array}{l}\text { Flack x determ } \\
2248 \\
-(\mathrm{I}-)] /[(\mathrm{I}+)+(\mathrm{I}-)] \text { (Parsons } \\
(2013) 249\end{array}$ & $\begin{array}{l}\text { mined using } \\
\qquad 1985 \\
\text {, Flack and Wag } \\
\text { 9-259). }\end{array}$ & $\begin{array}{c}1816 \\
\text { er, Acta Cryst B69 }\end{array}$ \\
\hline Absolute structure parameter & $0.020(6)$ & $0.020(6)$ & $0.003(9)$ & $0.006(9)$ \\
\hline
\end{tabular}




\begin{tabular}{|c|c|}
\hline & (23e) \\
\hline \multicolumn{2}{|l|}{ Crystal data } \\
\hline Chemical formula & $\mathrm{C}_{27} \mathrm{H}_{38} \mathrm{AuClN}_{2}$ \\
\hline$M_{\mathrm{r}}$ & 623.01 \\
\hline Crystal system, space group & Orthorhombic, $P 2_{1} 2_{1} 2_{1}$ \\
\hline Temperature (K) & 293 \\
\hline$a, b, c(\AA)$ & $9.032(4), 10.097(2), 28.845(8)$ \\
\hline$\alpha, \beta, \gamma\left(^{\circ}\right)$ & $90,90,90$ \\
\hline$V\left(\AA^{3}\right)$ & $2630.7(14)$ \\
\hline$Z$ & 4 \\
\hline Radiation type & Mo $K \alpha$ \\
\hline$\mu\left(\mathrm{mm}^{-1}\right)$ & 5.71 \\
\hline Crystal size (mm) & $0.3 \times 0.25 \times 0.1$ \\
\hline \multicolumn{2}{|l|}{ Data collection } \\
\hline Diffractometer & Enraf Nonius MACH3 \\
\hline Absorption correction & $\begin{array}{l}\psi \text { scan North A.C.T., Phillips D.C. \& Mathews F.S. (1968) Acta. Cryst. } \\
\text { A24, } 351 \text { Number of } \psi \text { scan sets used was } 4 \text { Theta correction was } \\
\text { applied. Averaged transmission function was used. Fourier smoothing - } \\
\text { Window value } 5\end{array}$ \\
\hline$T_{\min }, T_{\max }$ & $0.188,0.308$ \\
\hline $\begin{array}{l}\text { No. of measured, independent and } \\
\text { observed }[I>2 \sigma(I)] \text { reflections }\end{array}$ & $5498,5498,3171$ \\
\hline$R_{\text {int }}$ & 0.07 \\
\hline$(\sin \theta / \lambda)_{\max }\left(\AA^{-1}\right)$ & 0.603 \\
\hline \multicolumn{2}{|l|}{ Refinement } \\
\hline$R\left[F^{2}>2 \sigma\left(F^{2}\right)\right], w R\left(F^{2}\right), S$ & $0.078,0.185,0.97$ \\
\hline No. of reflections & 5498 \\
\hline No. of parameters & 284 \\
\hline No. of restraints & 0 \\
\hline $\mathrm{H}$-atom treatment & H-atom parameters constrained \\
\hline$\left.\Delta\rangle_{\max }, \Delta\right\rangle_{\min }\left(\mathrm{e} \AA^{-3}\right)$ & $1.17,-1.62$ \\
\hline Absolute structure & $\begin{array}{l}\text { Flack x determined using } 797 \text { quotients [(I+)-(I-)]/[(I+)+(I-)] (Parsons } \\
\text { and Flack (2004), Acta Cryst. A60, s61). }\end{array}$ \\
\hline Absolute structure parameter & $-0.07(3)$ \\
\hline
\end{tabular}




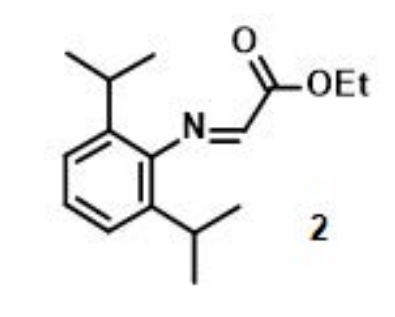

\section{$1 \mathrm{H}$ and $13 \mathrm{C}$ NMR spectra of the prepared compounds}

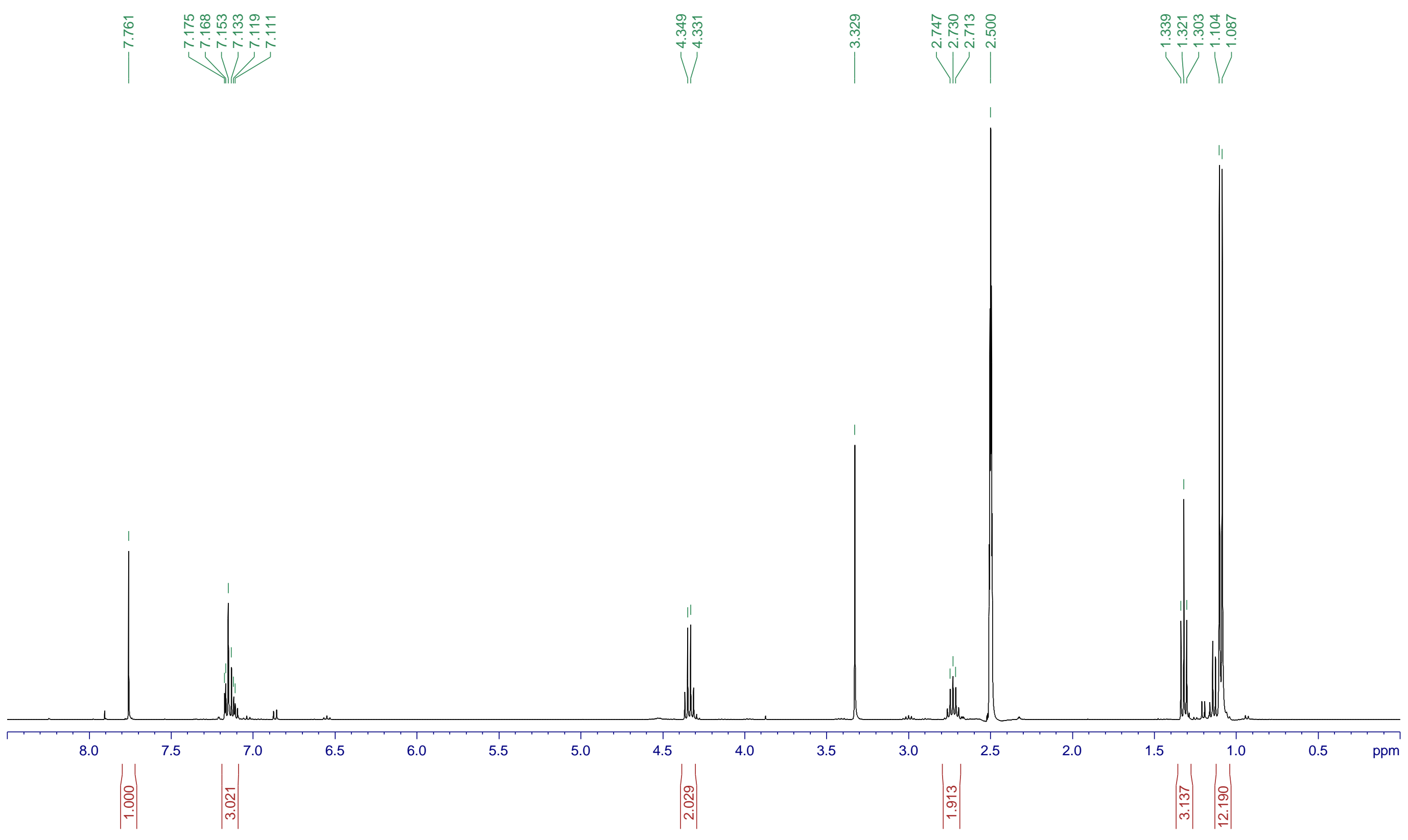


$\underbrace{N}=$

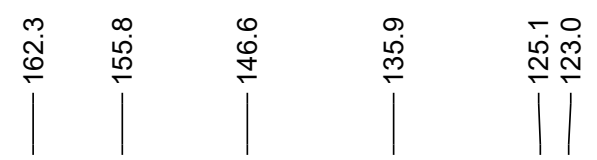

$\stackrel{m}{\stackrel{m}{N}} \stackrel{\stackrel{0}{+}}{\mid}$

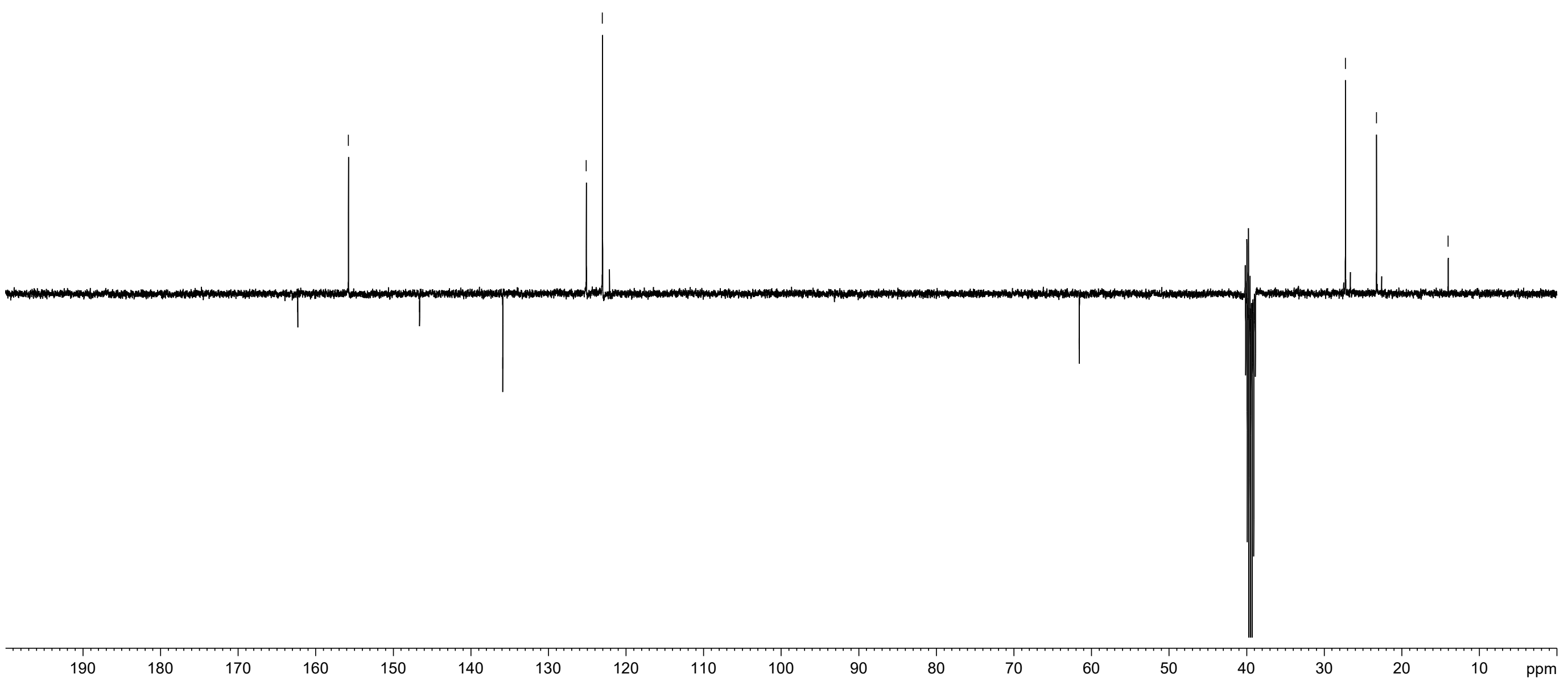



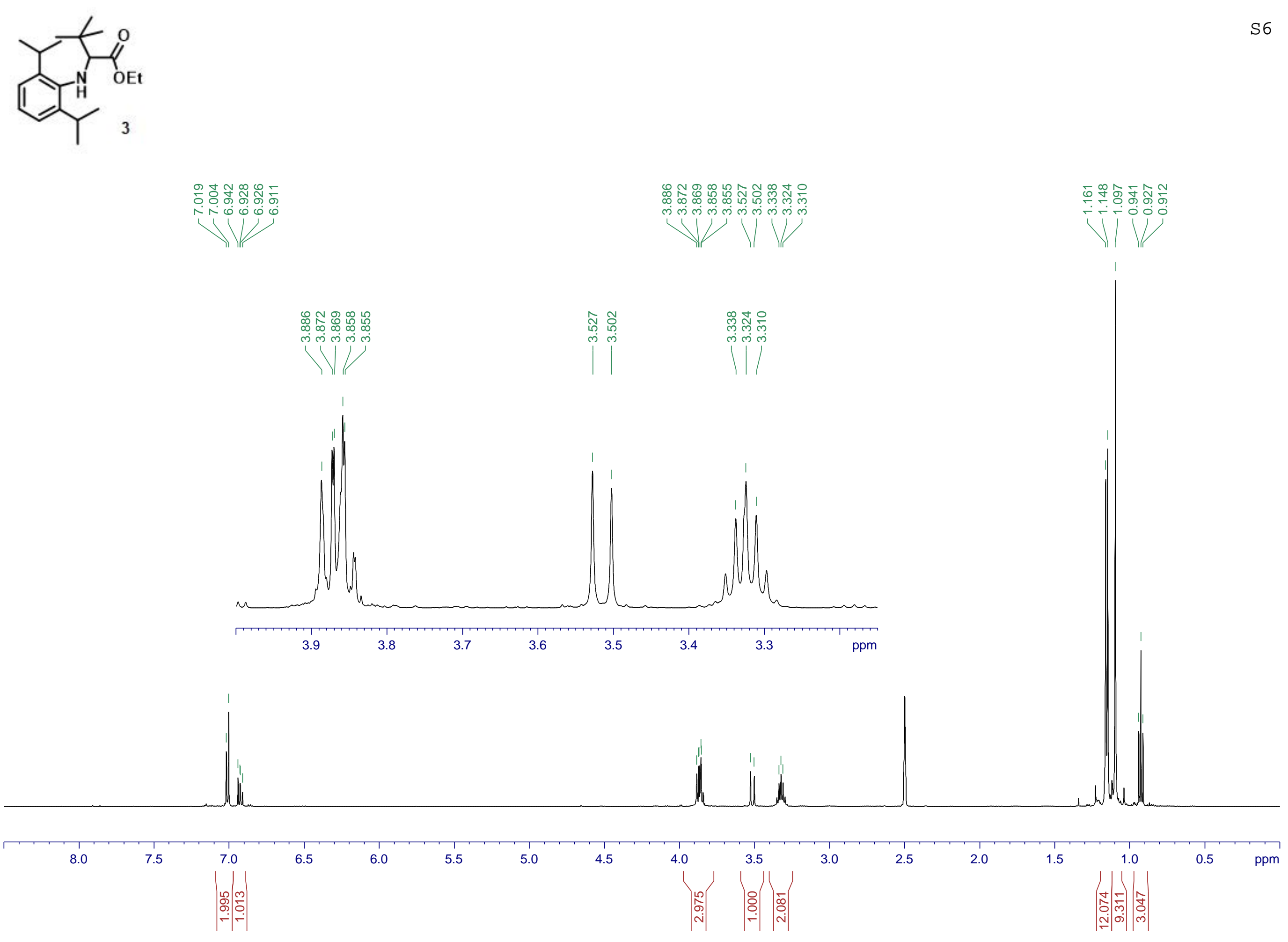


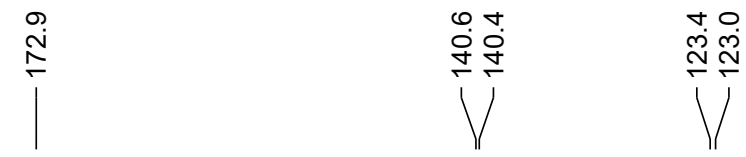

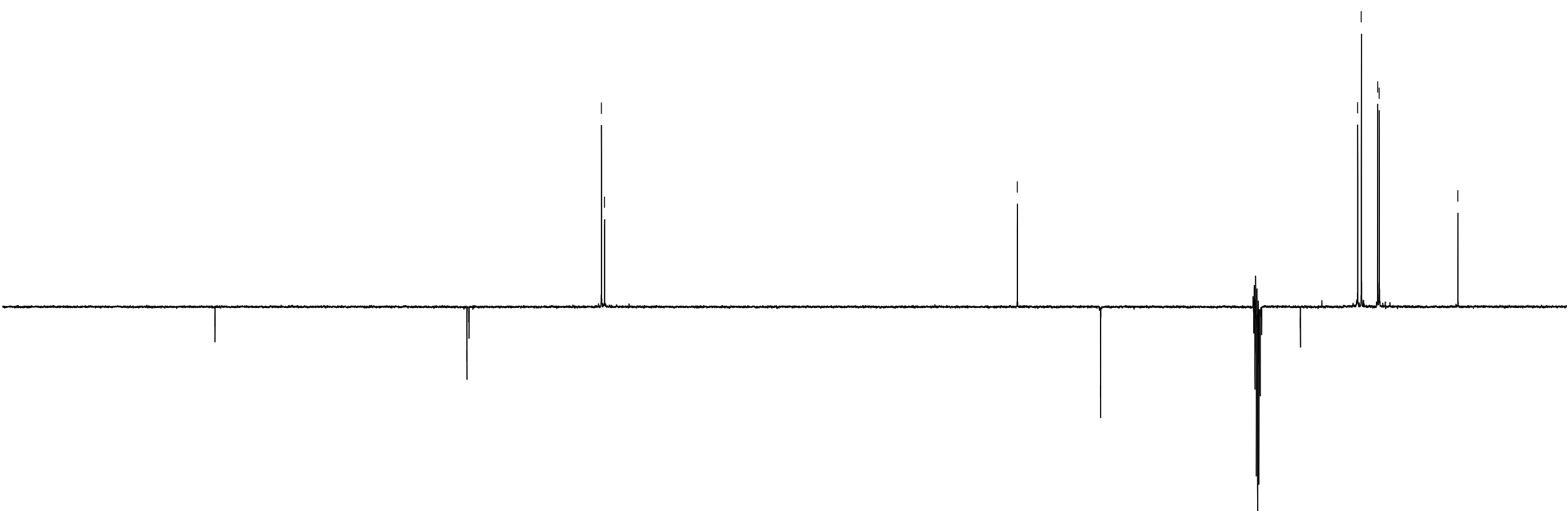




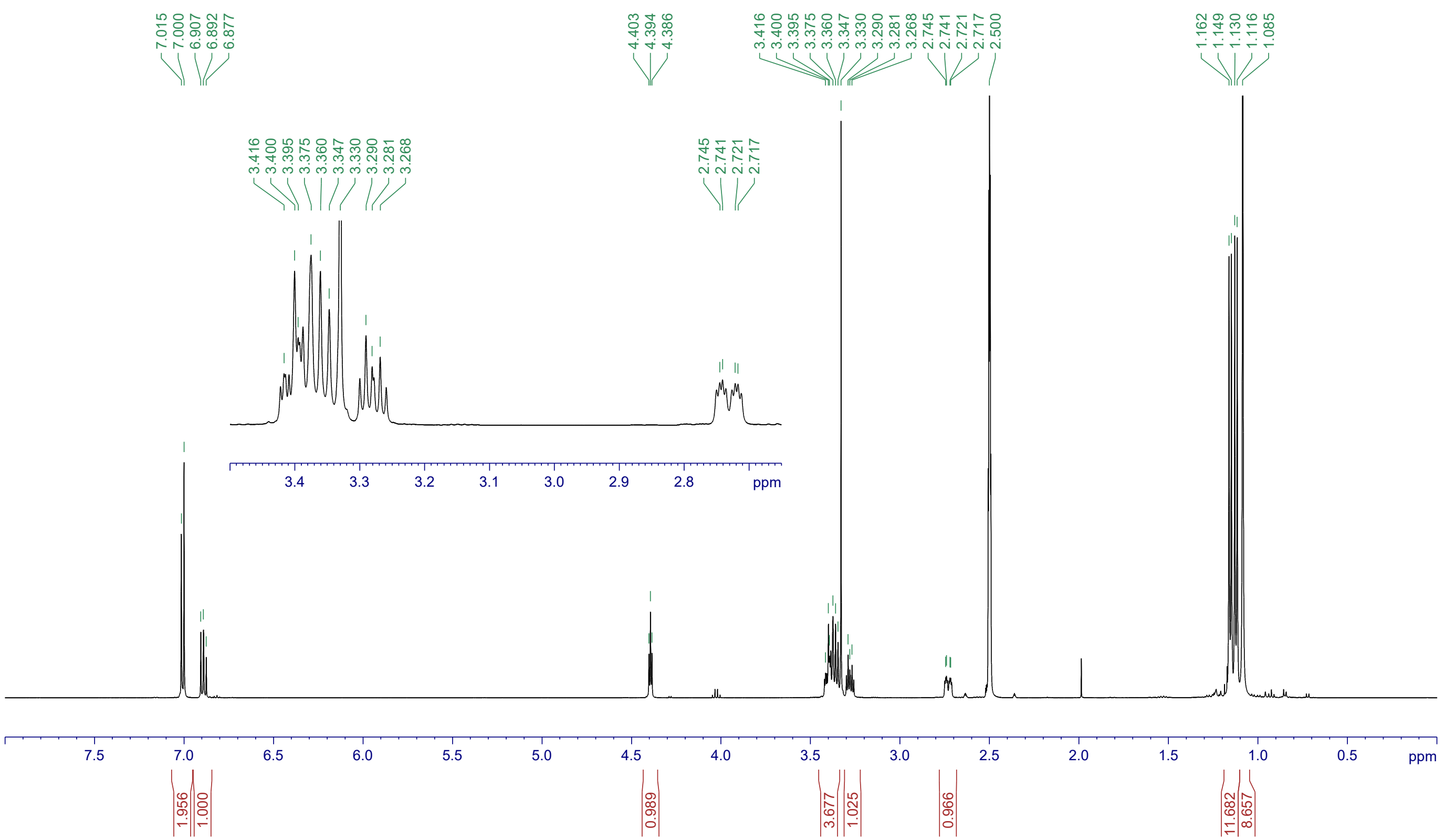



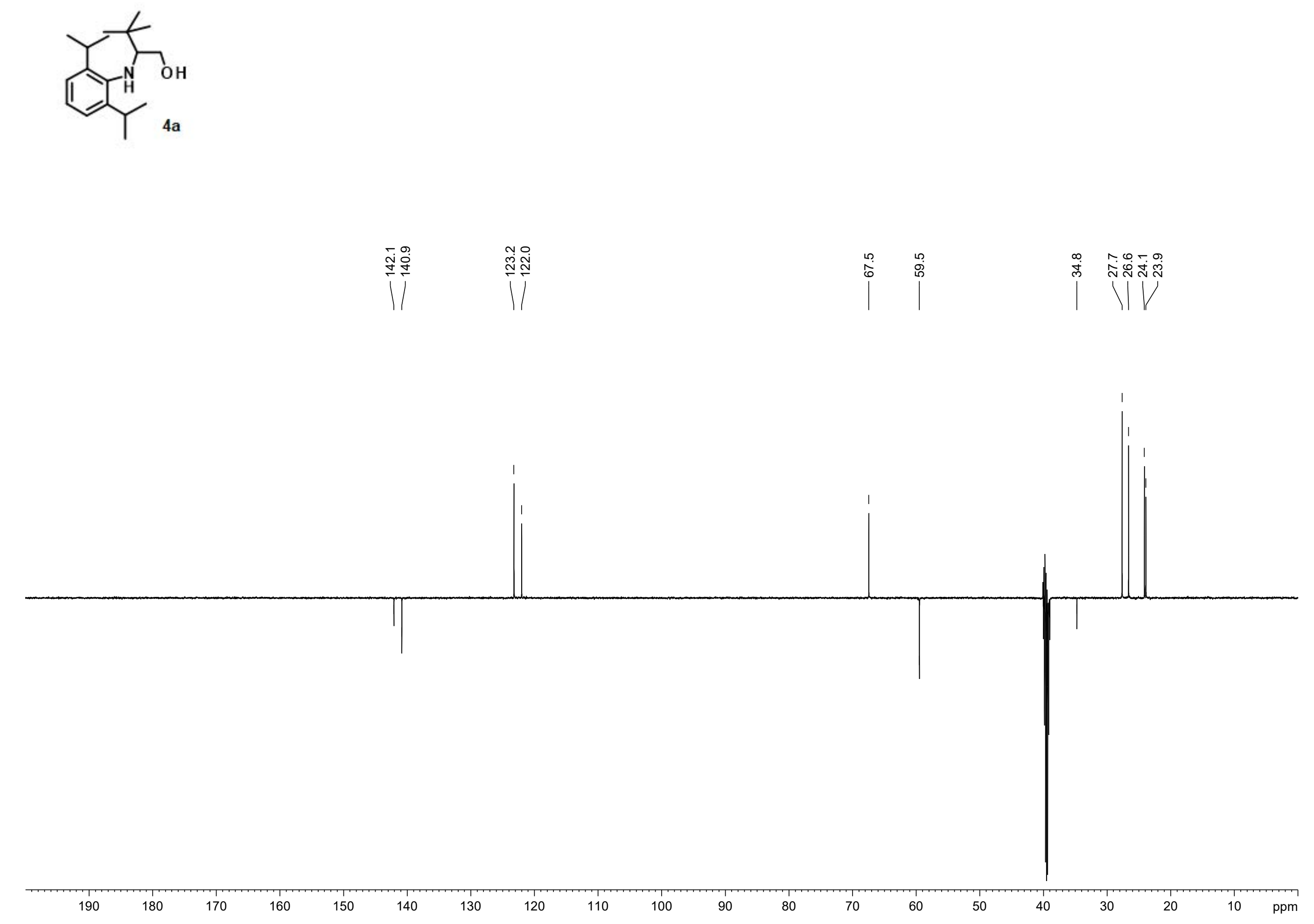


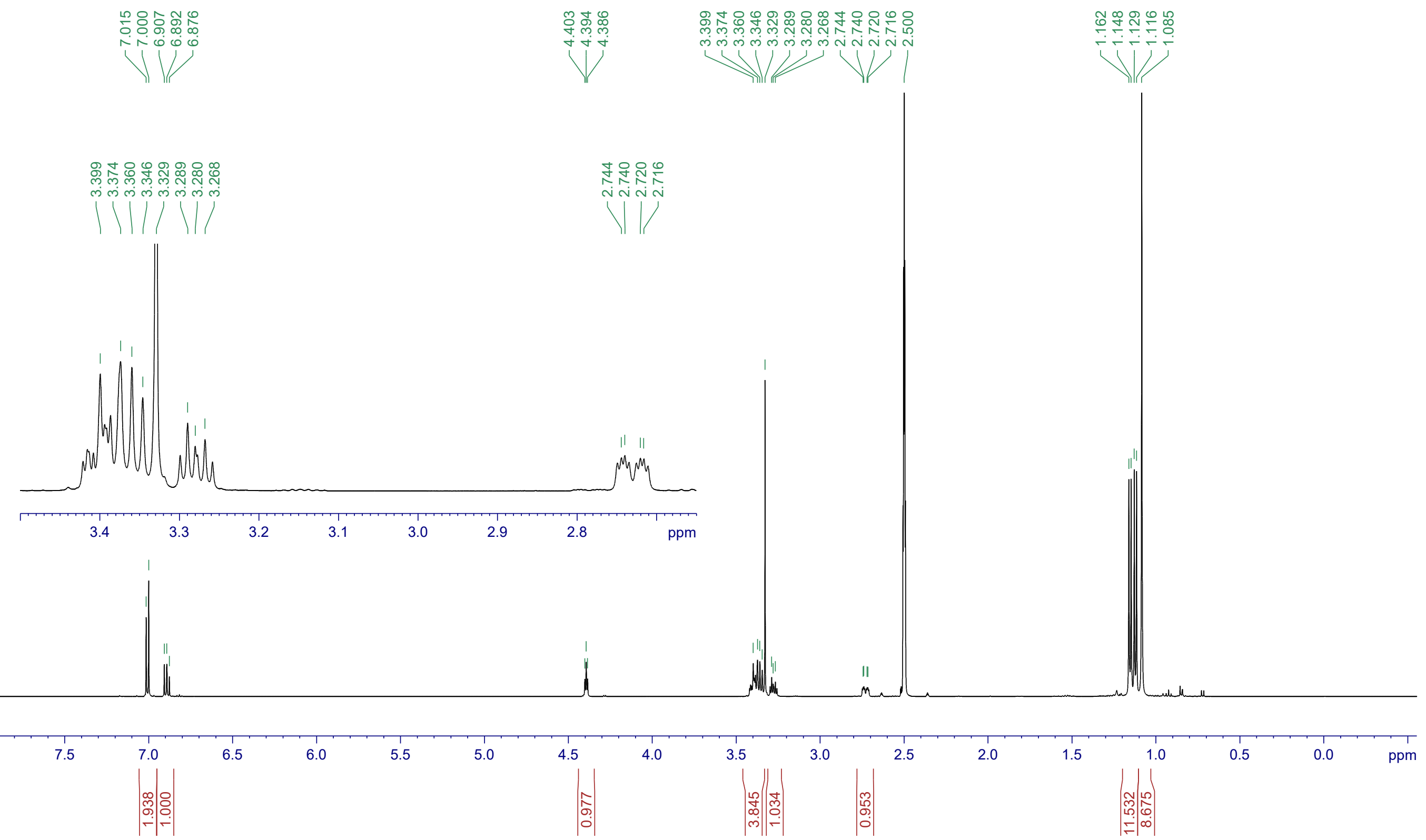




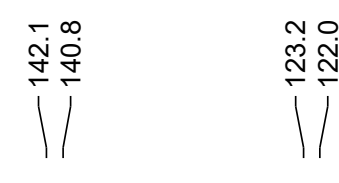

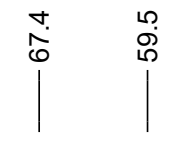

峁

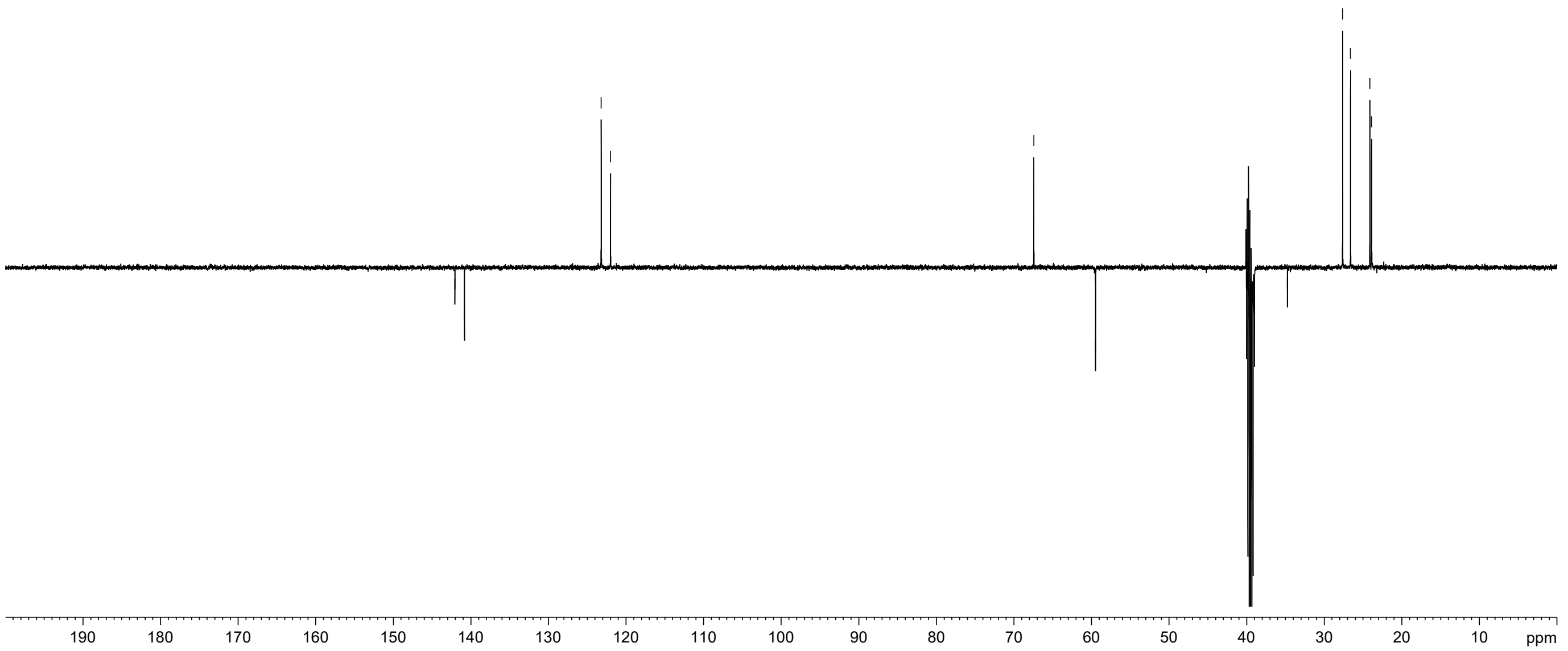



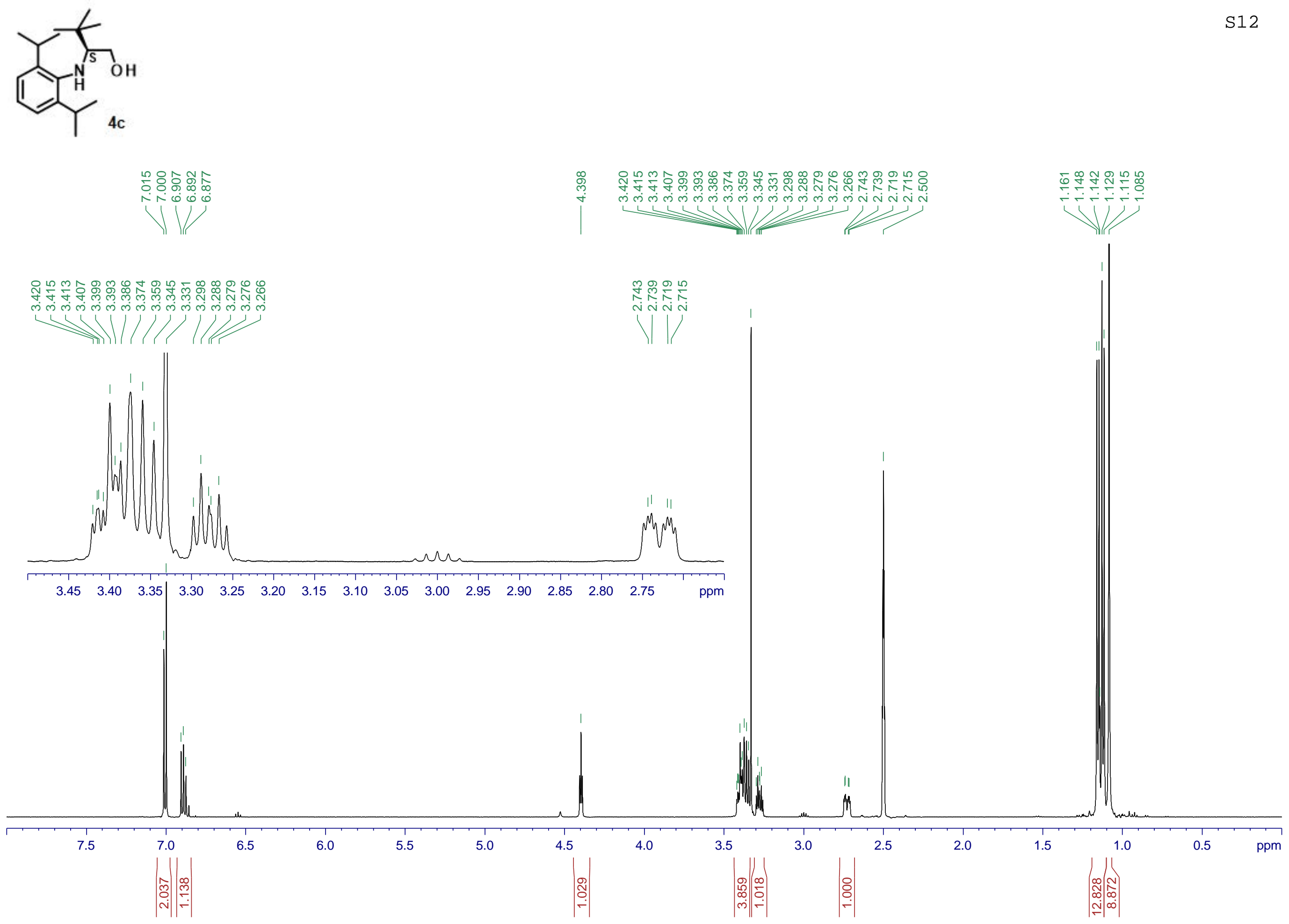



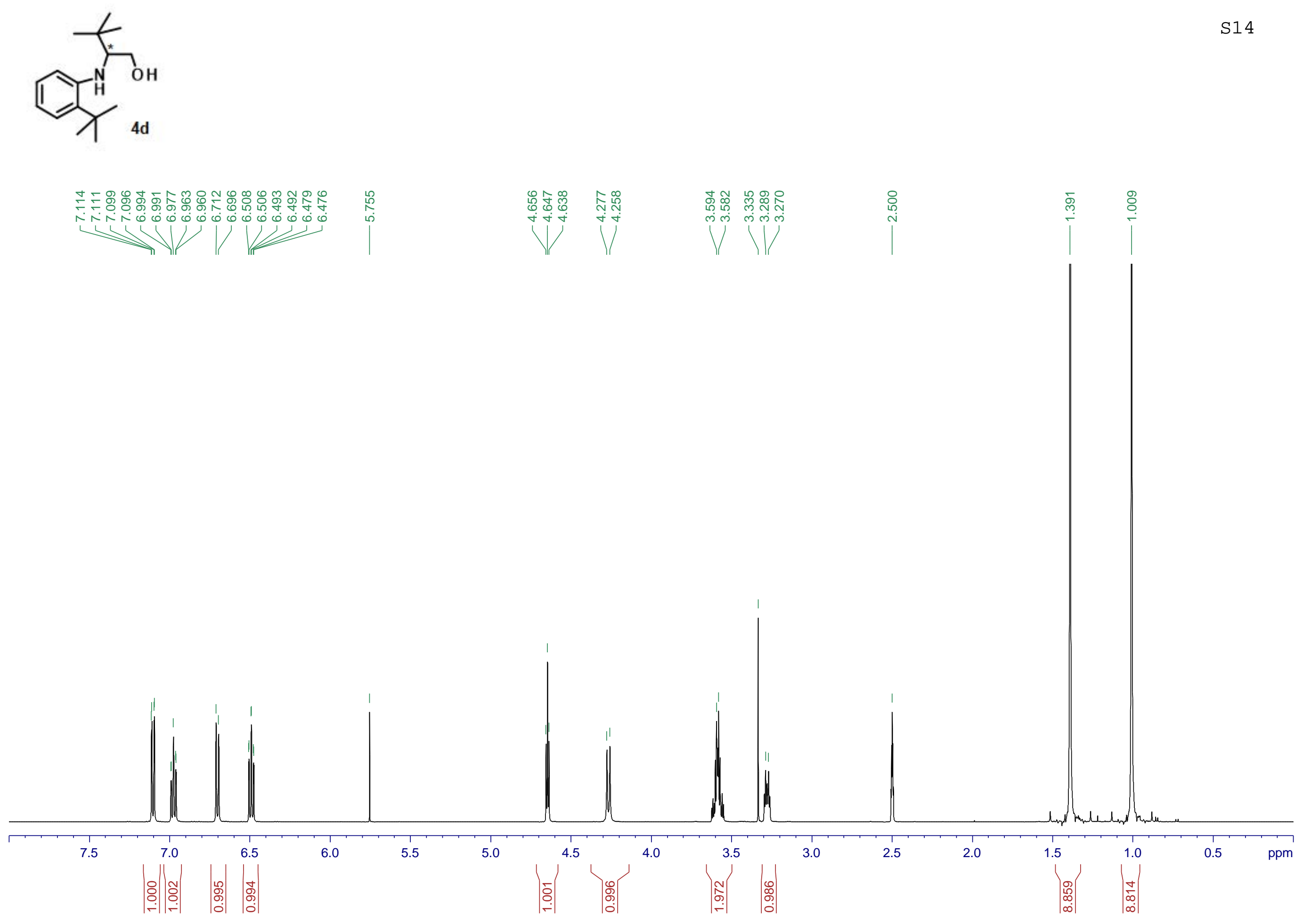


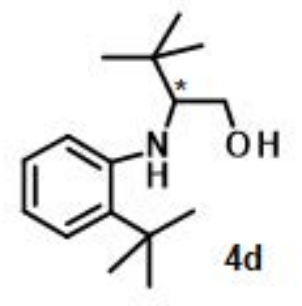

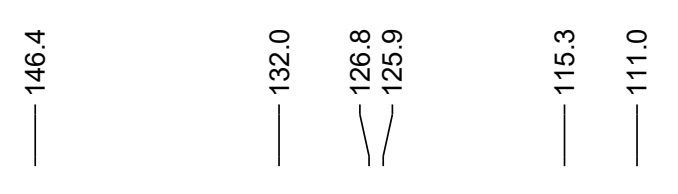

||

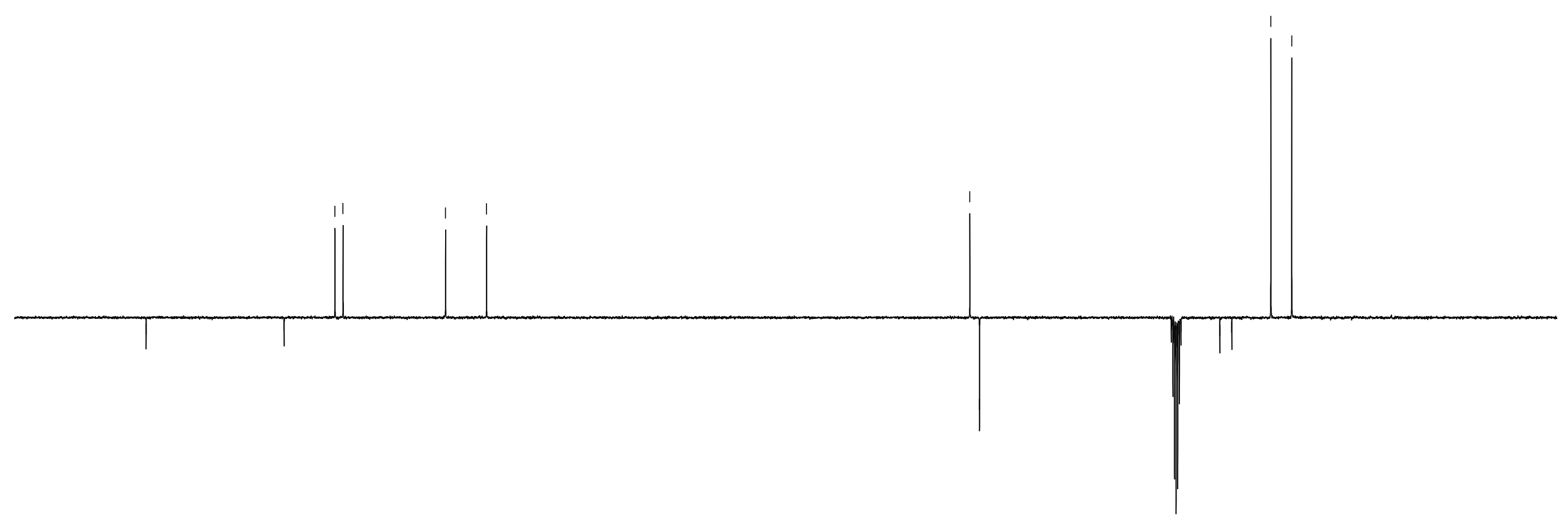




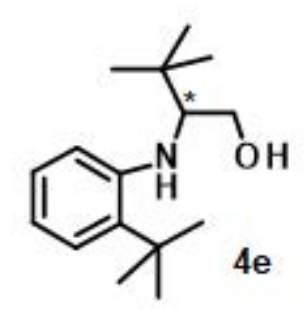

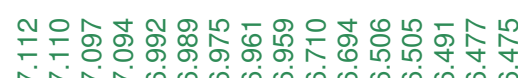

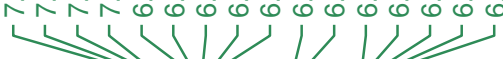

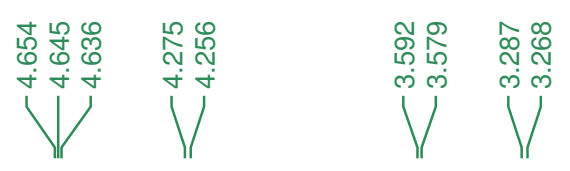

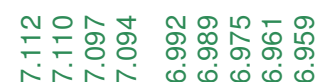

î
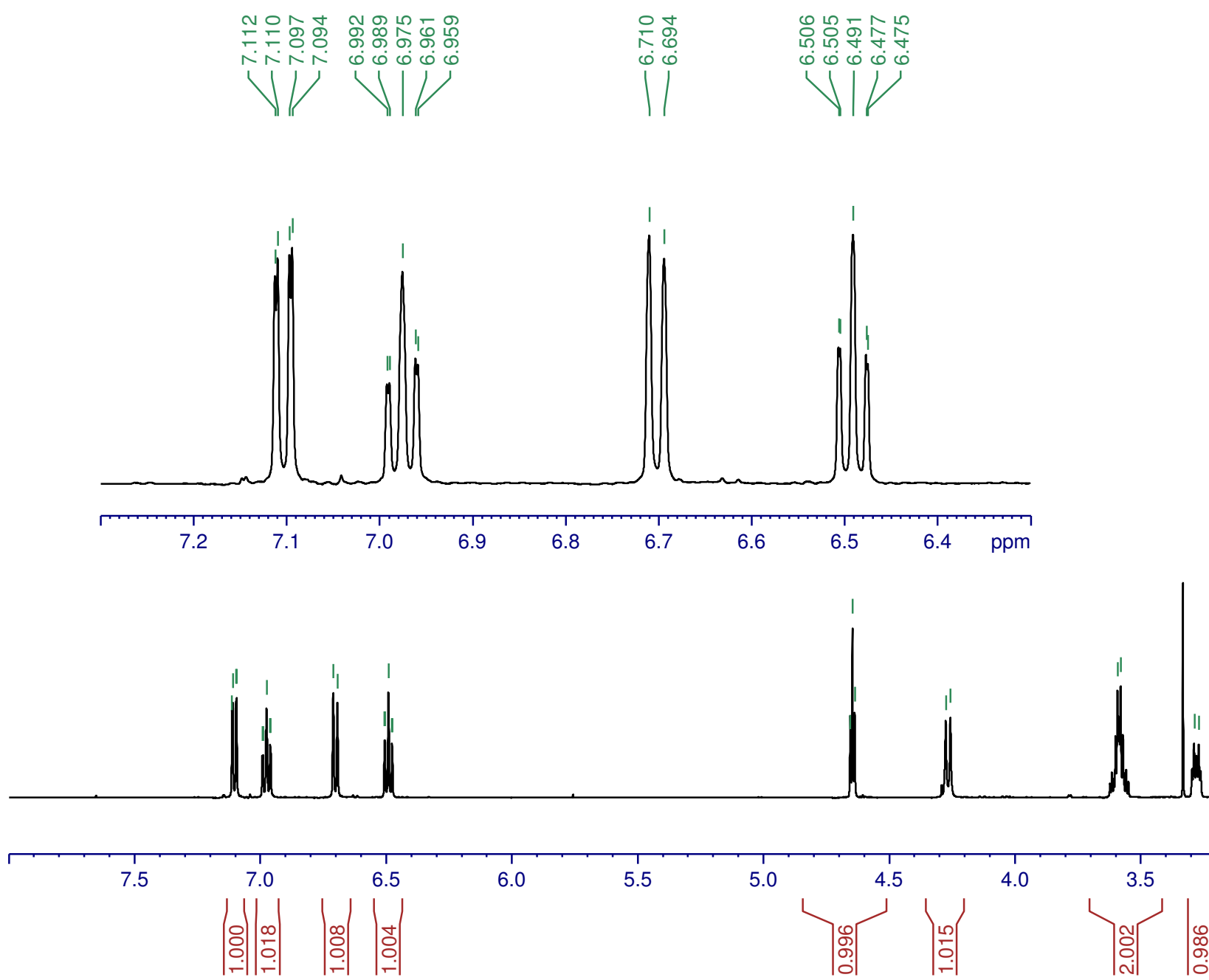

4.0

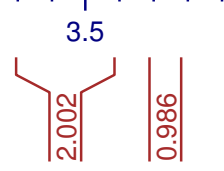

3.0

2.5

2.0

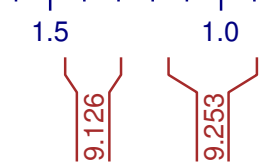




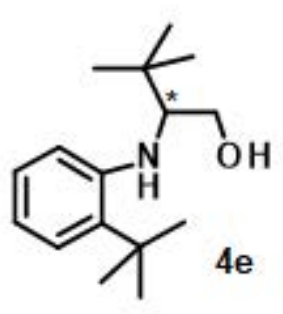

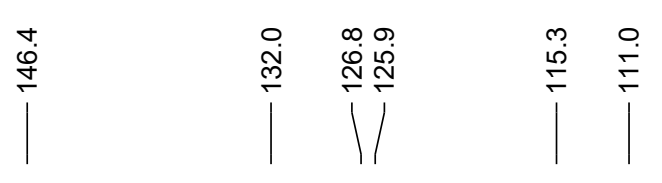

웅요

11

용ํํ

|l|

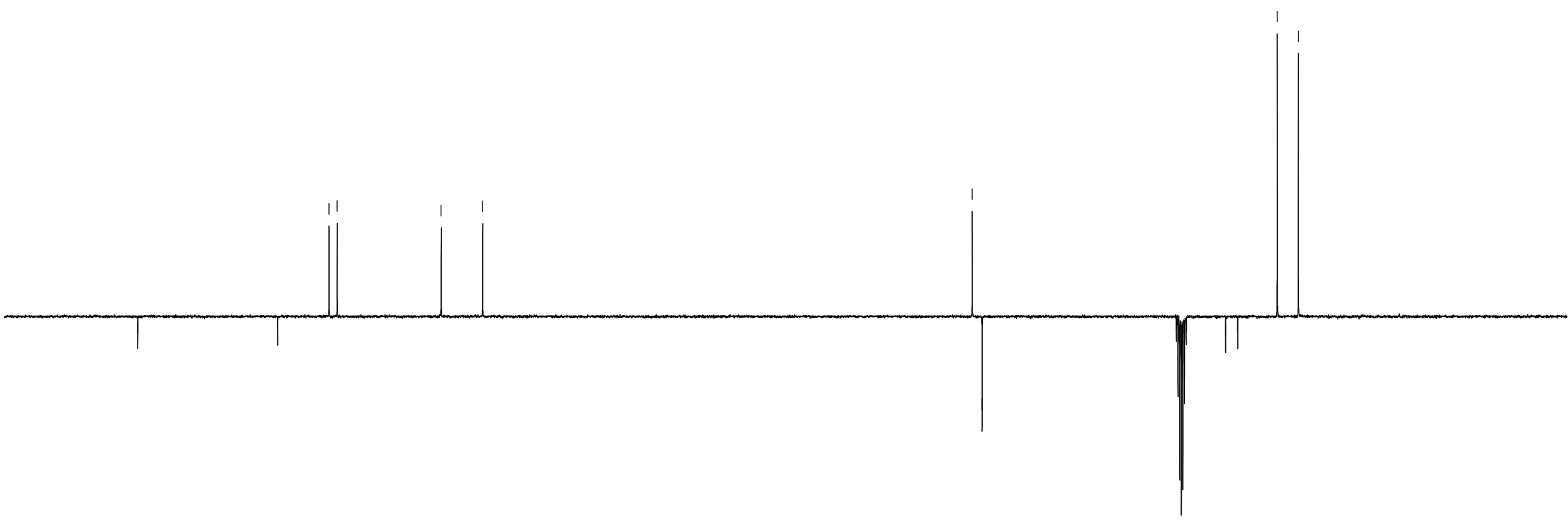



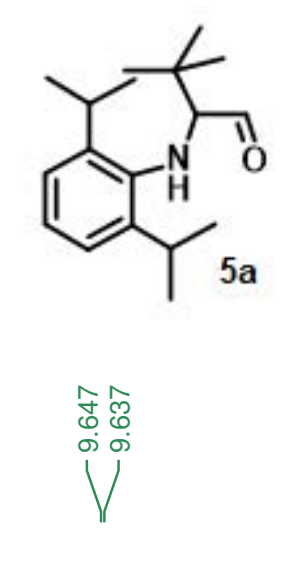

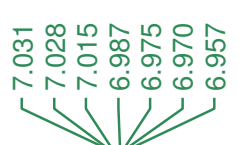
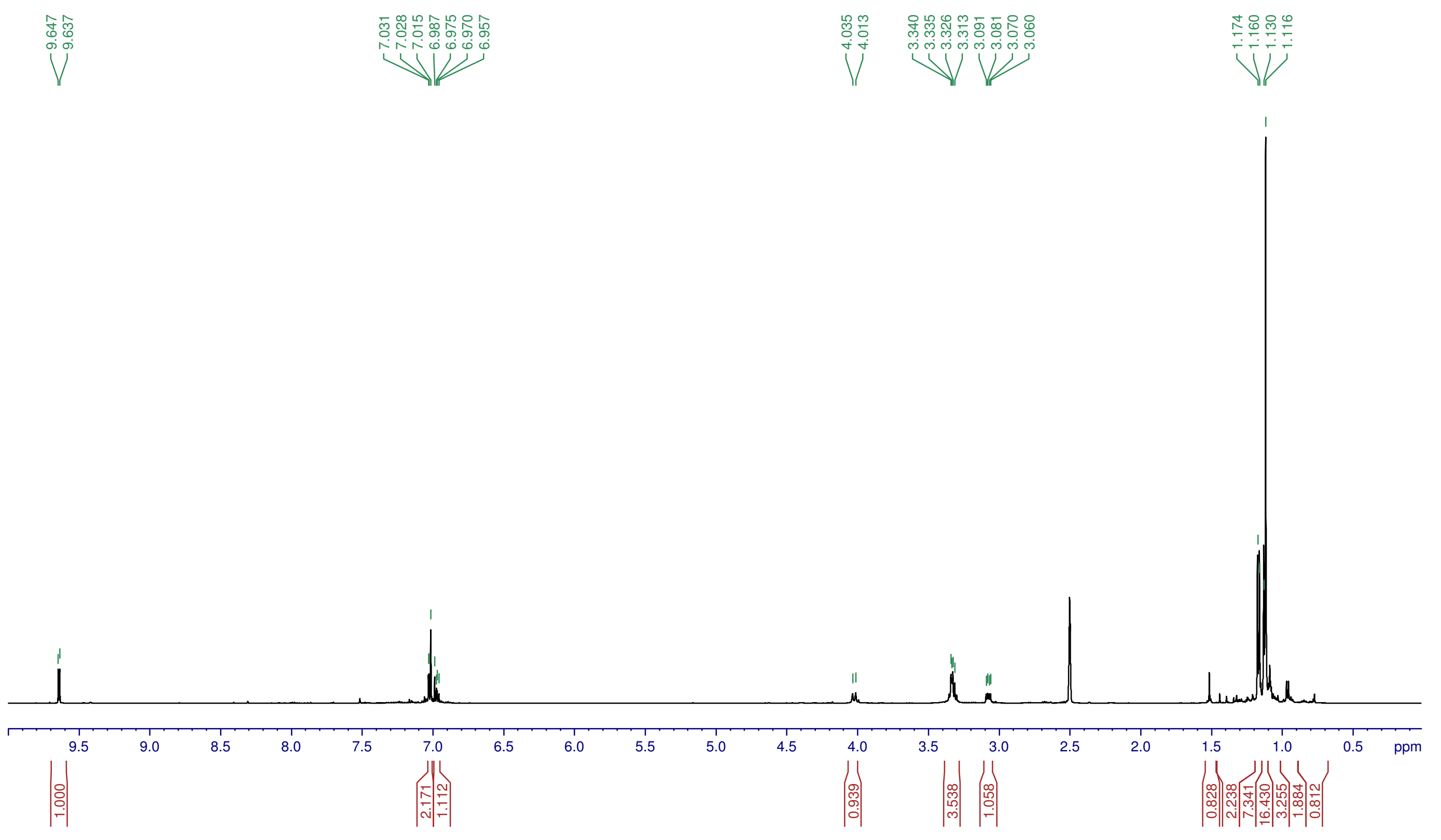

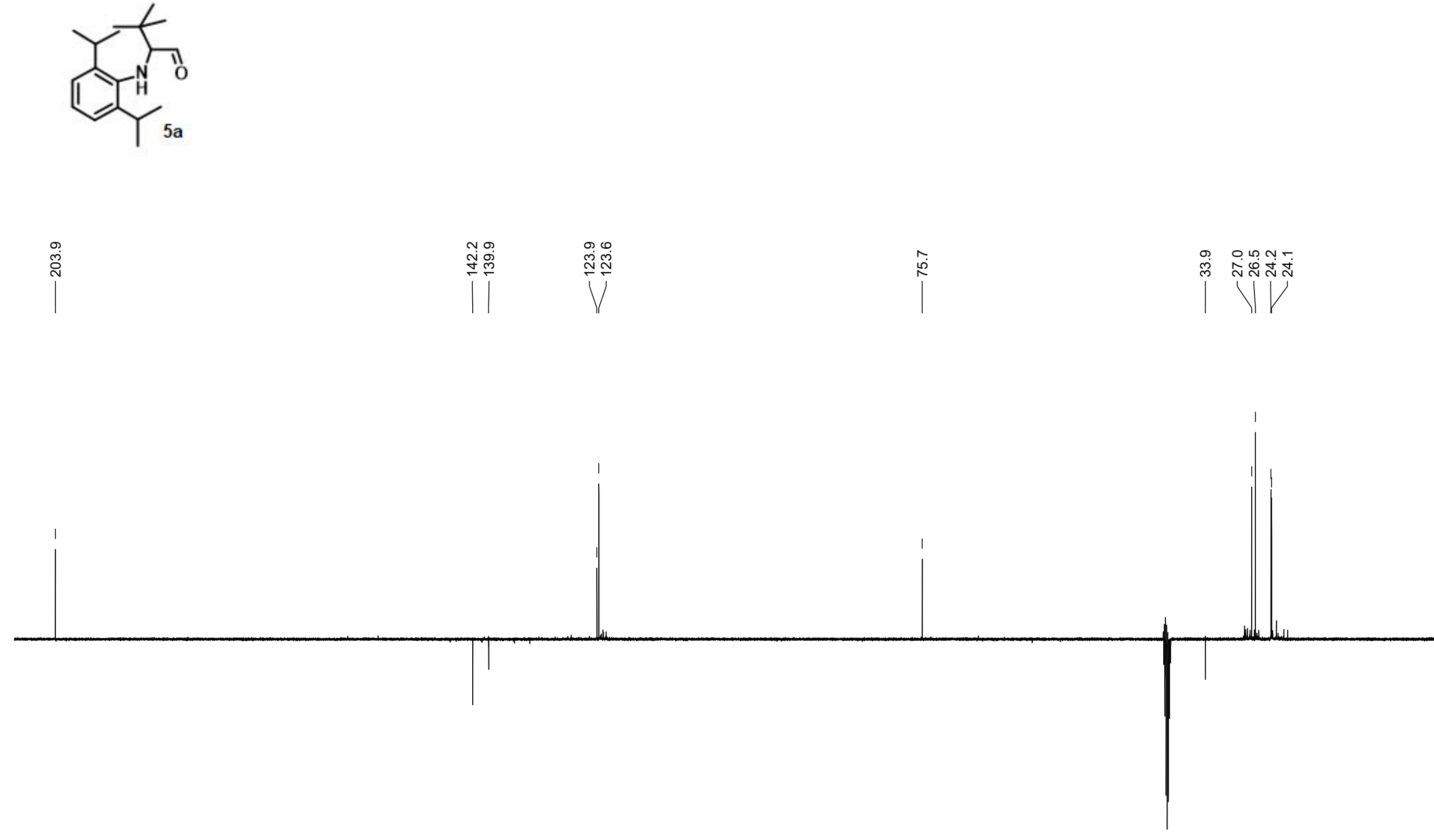

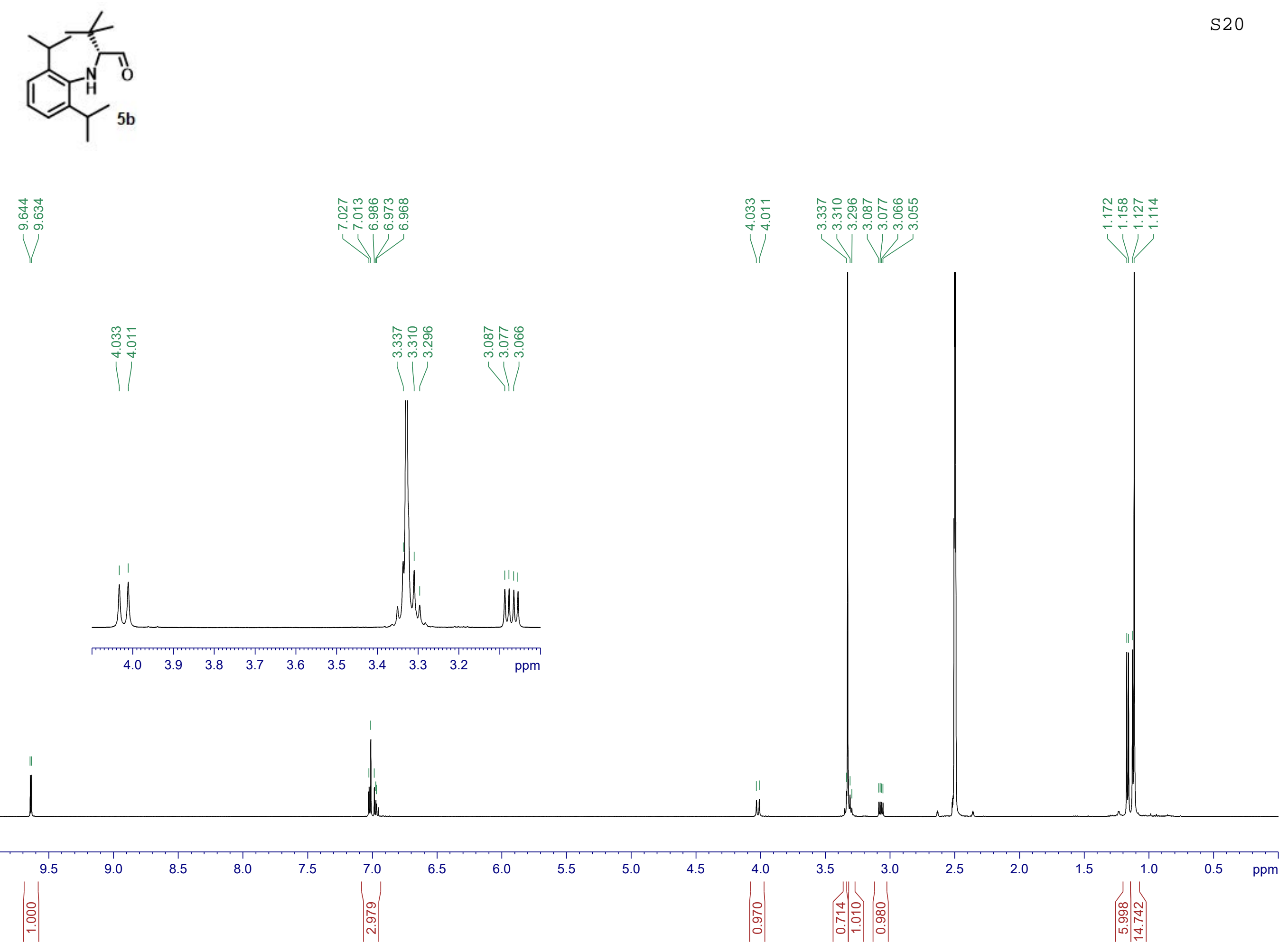

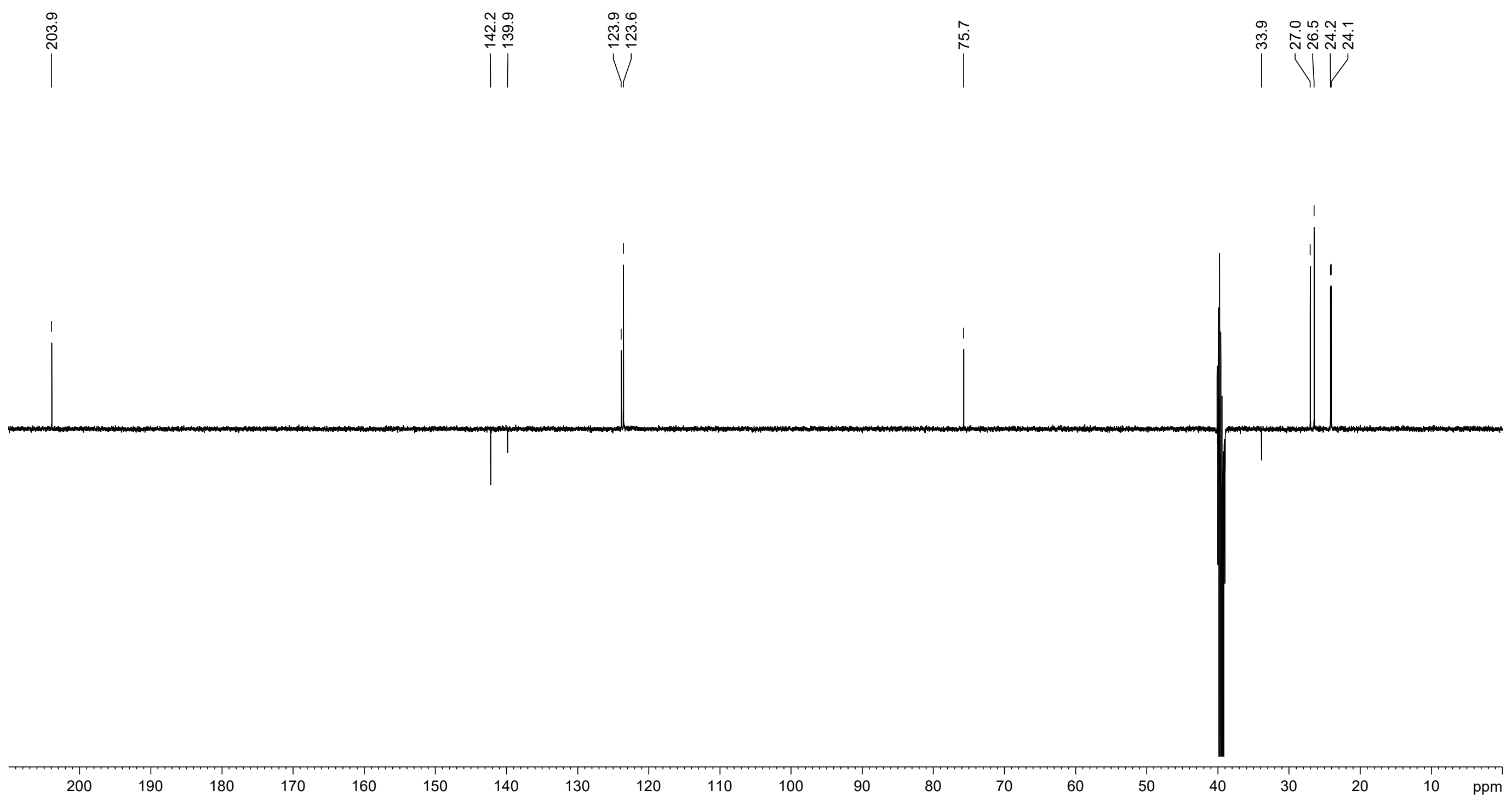

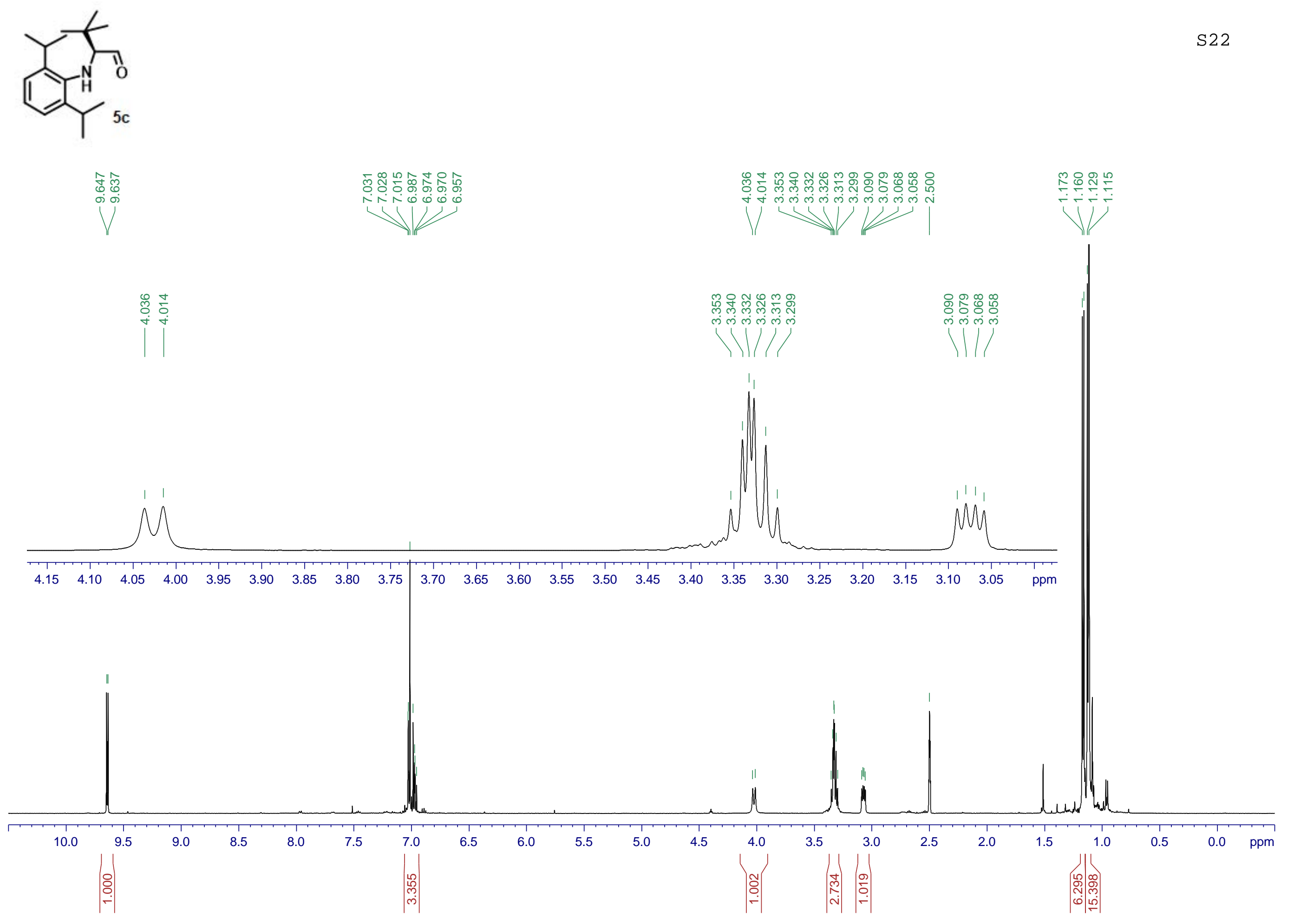


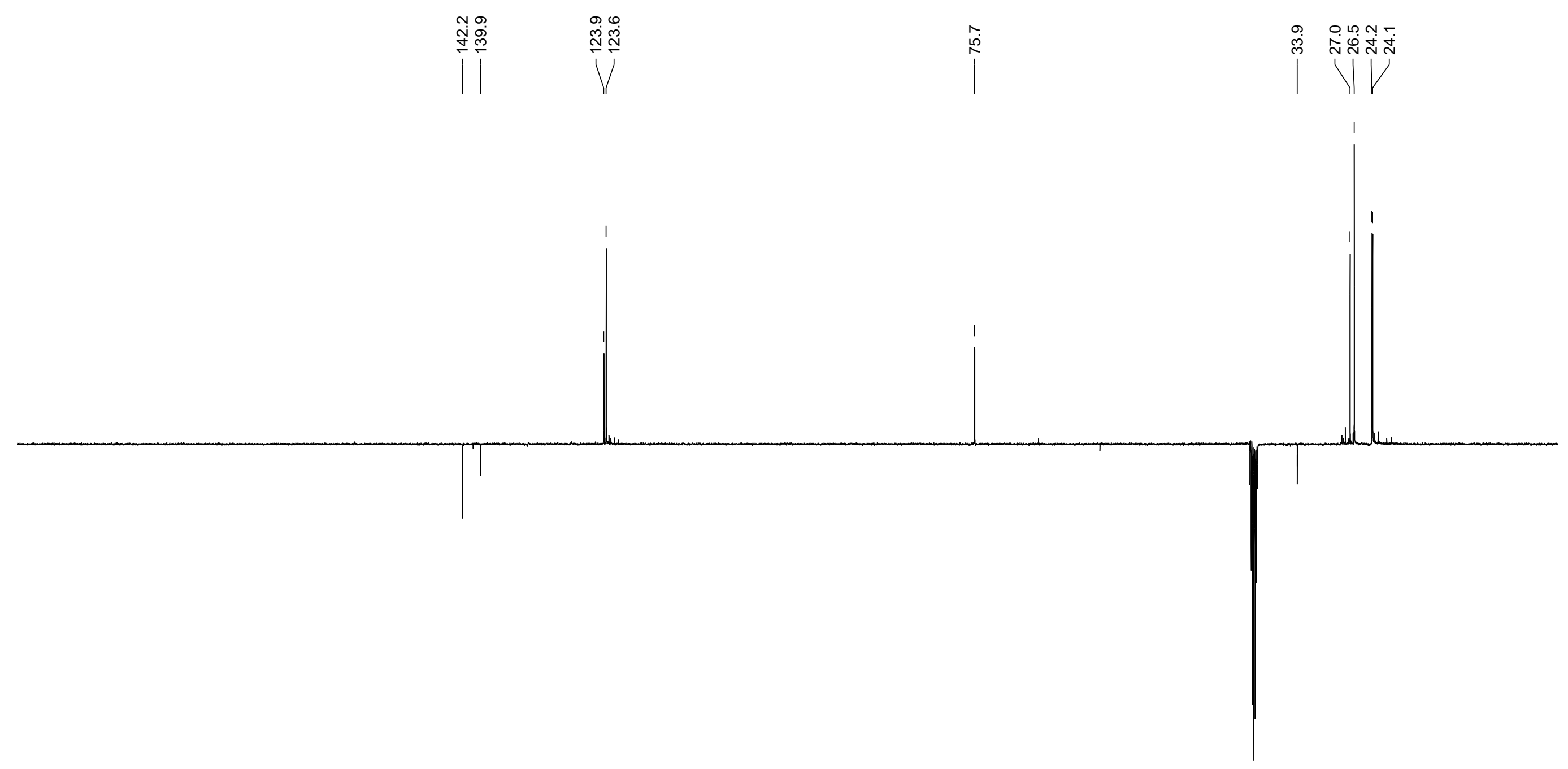



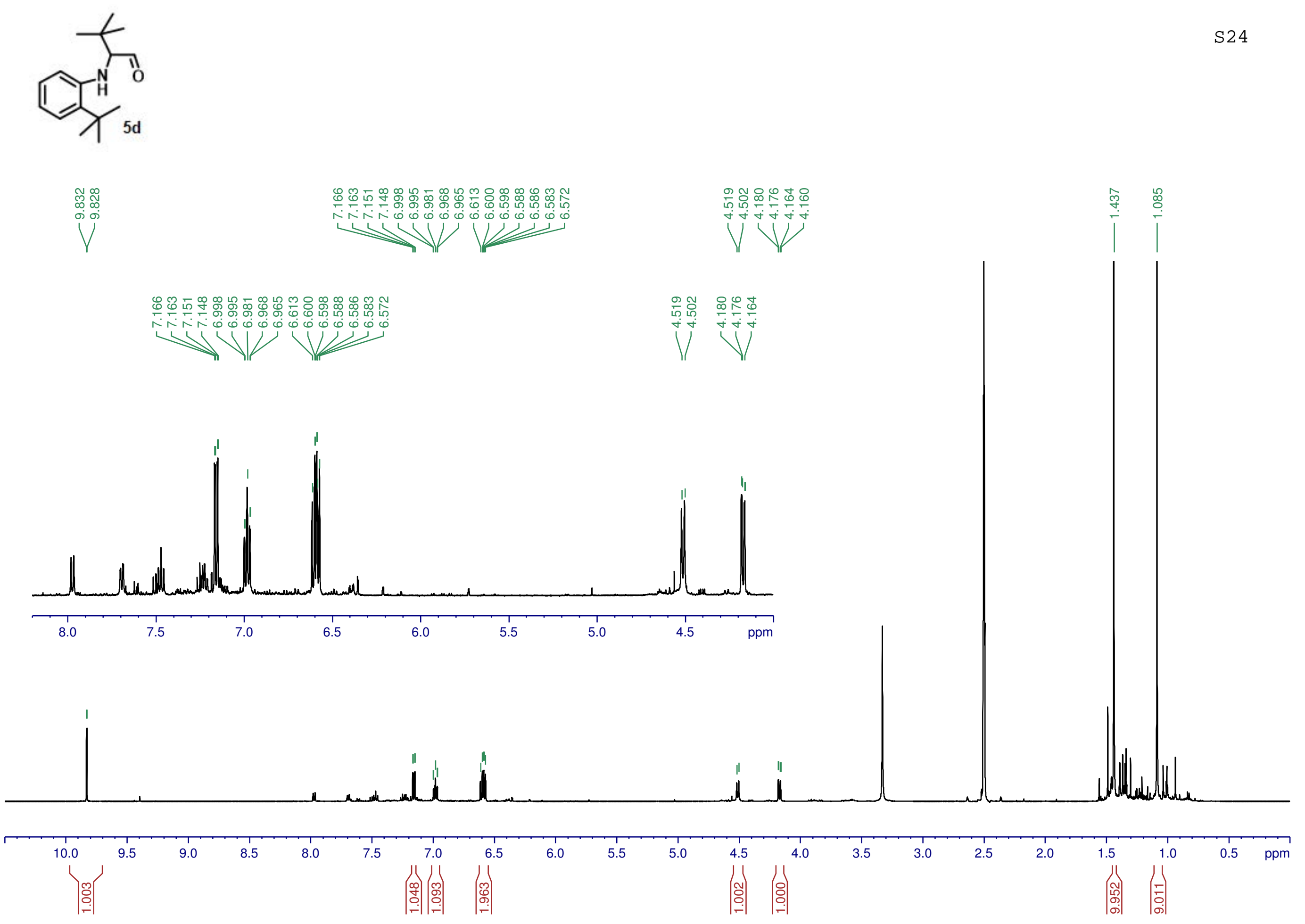

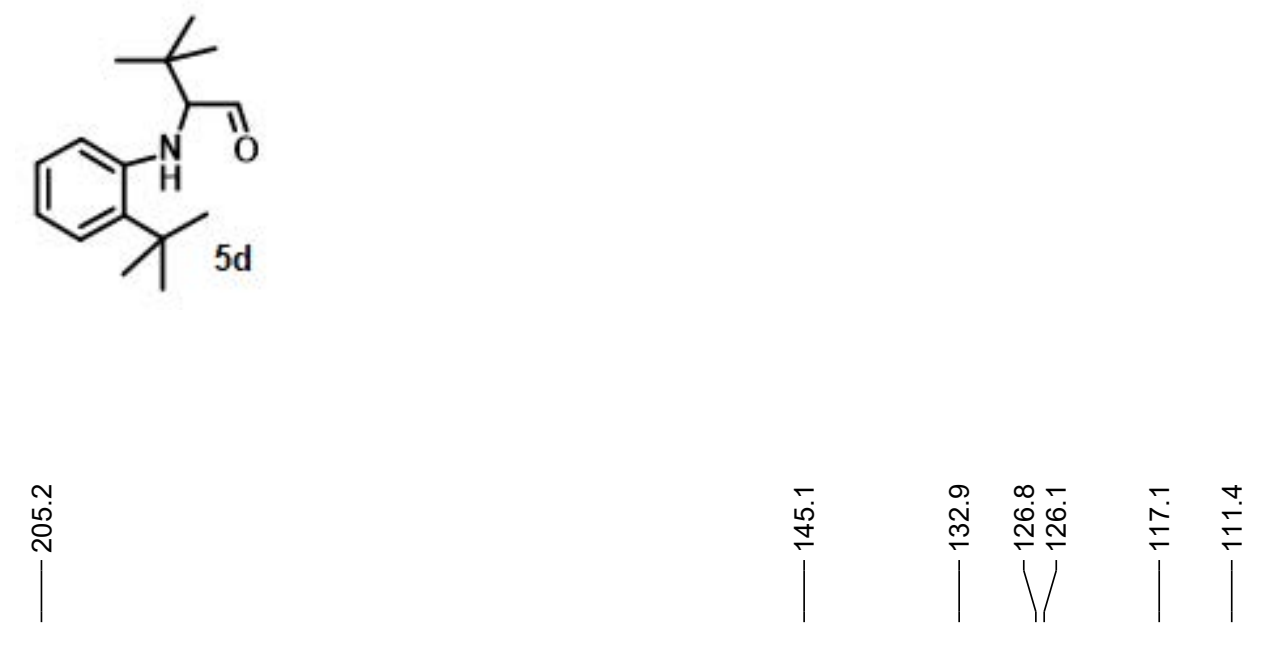

$\left.\right|^{\infty}$

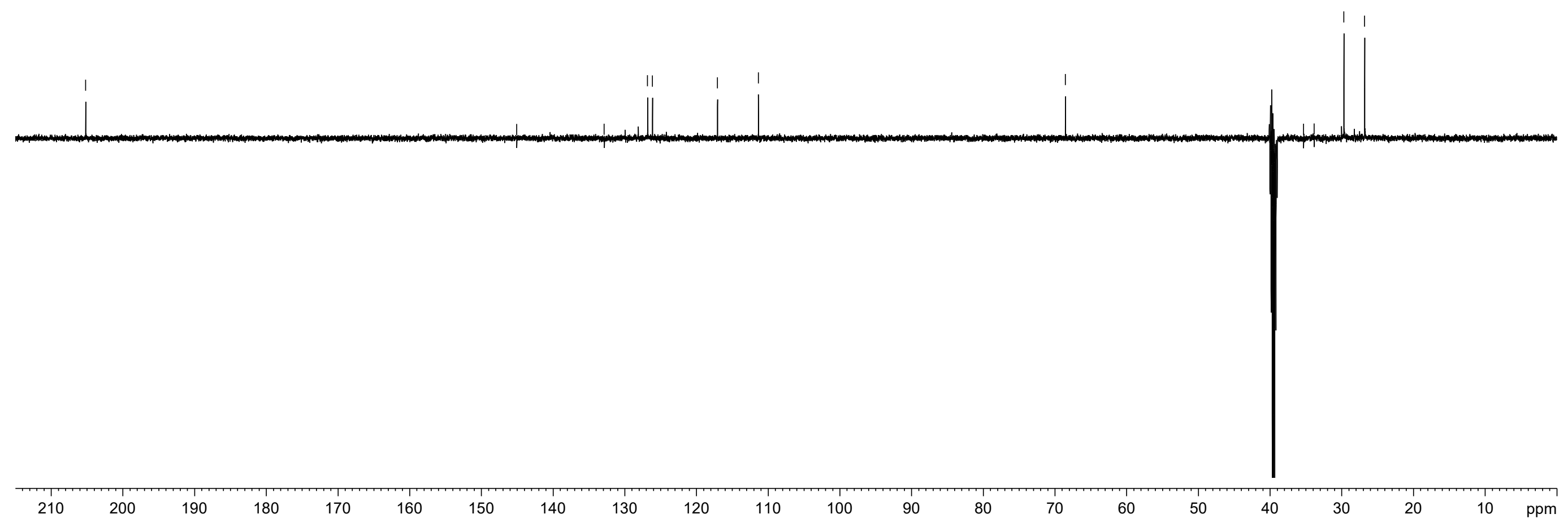



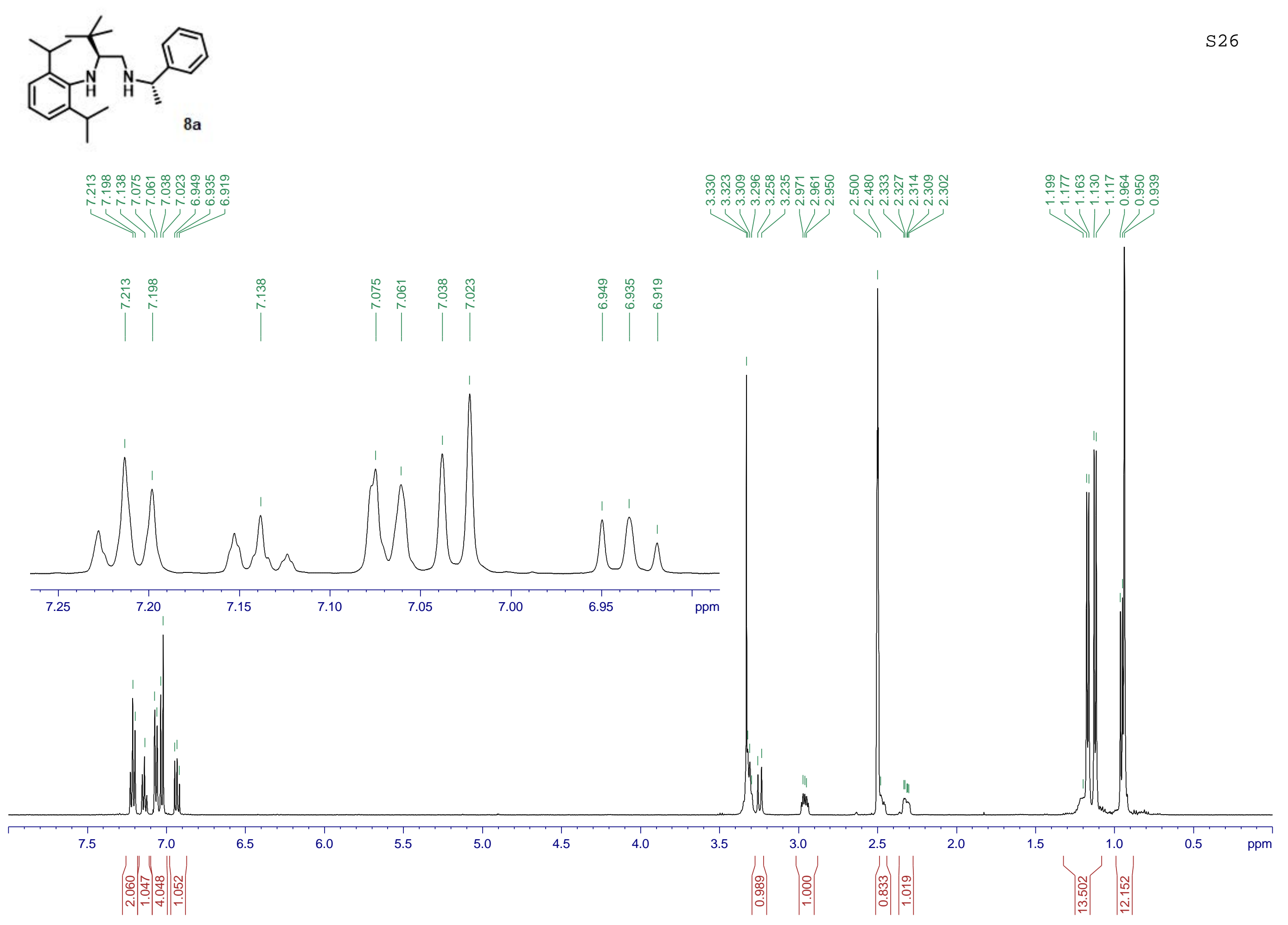

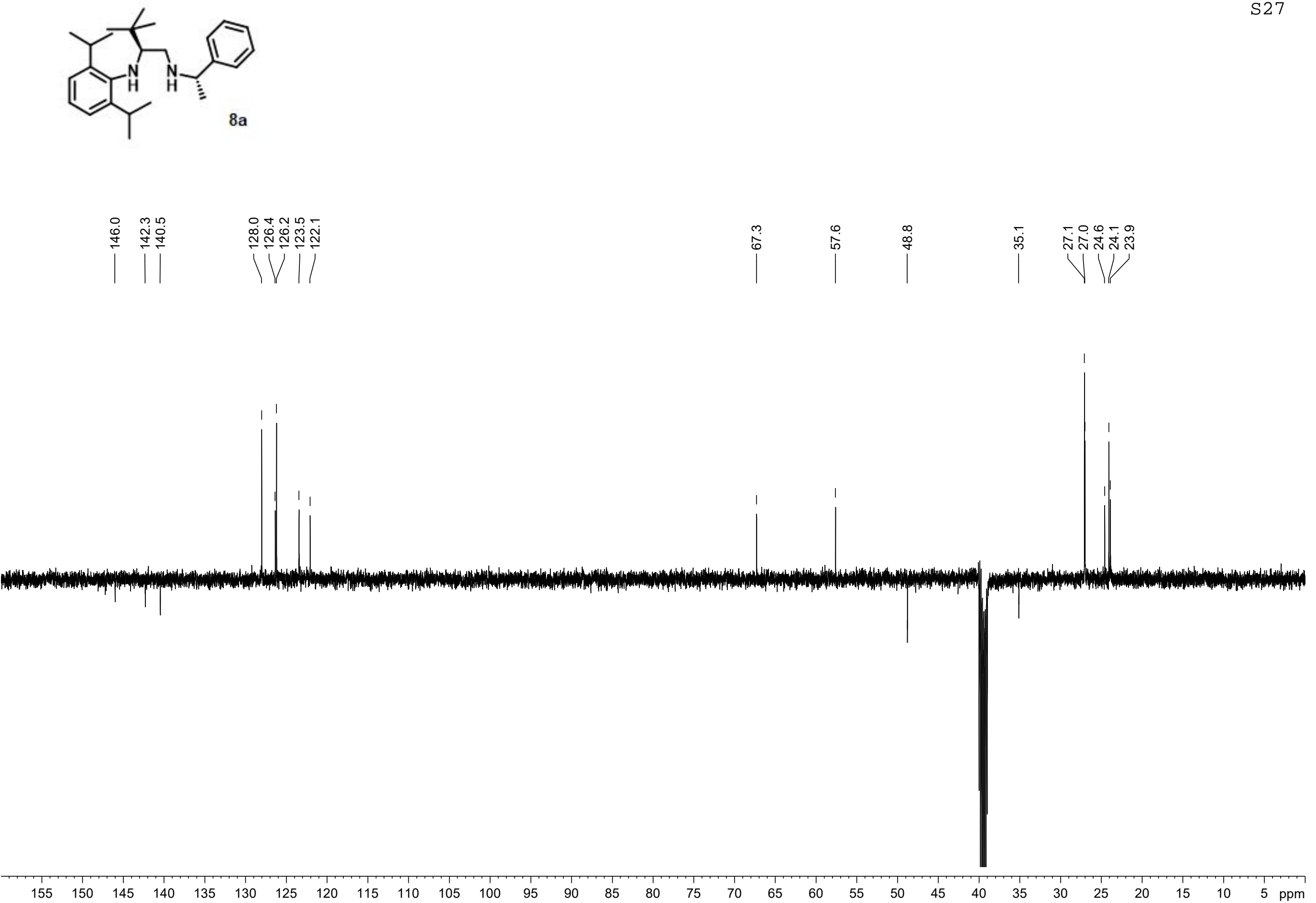




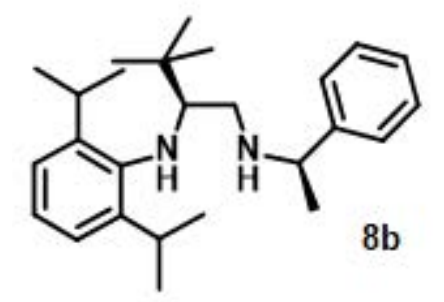

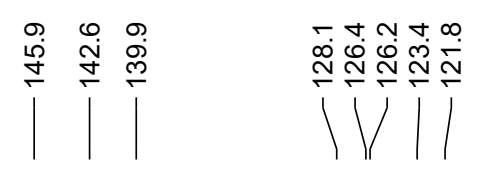


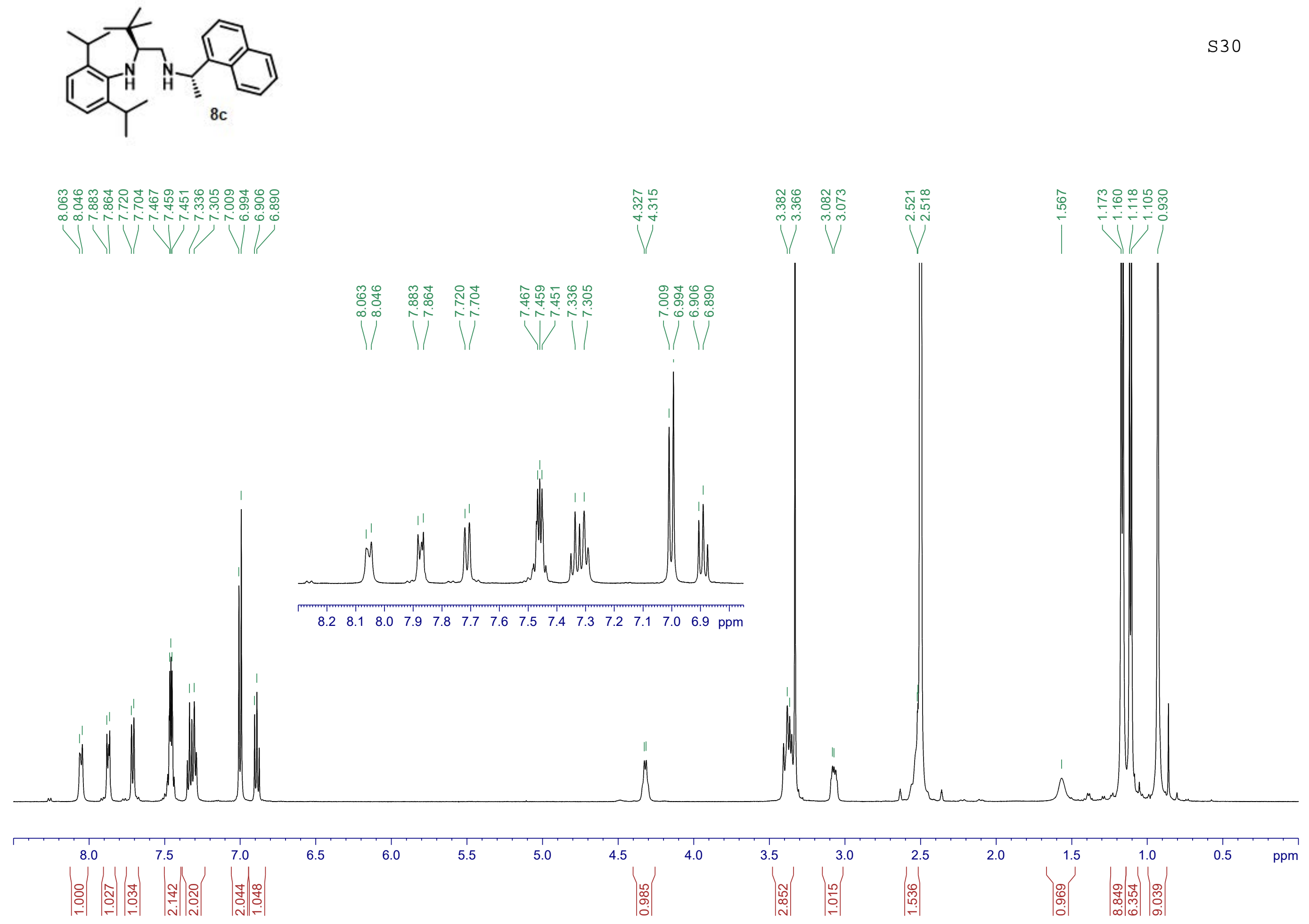


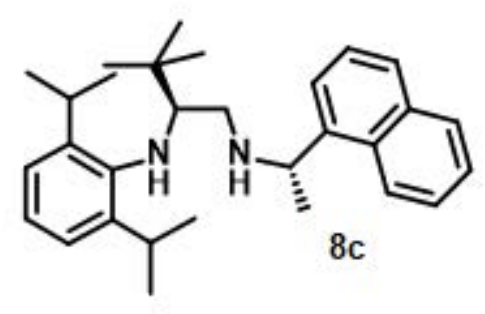

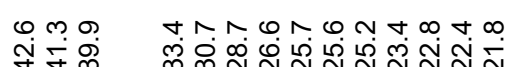

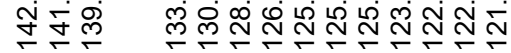

HIN11/1/

$\mid$

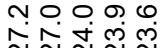

VV
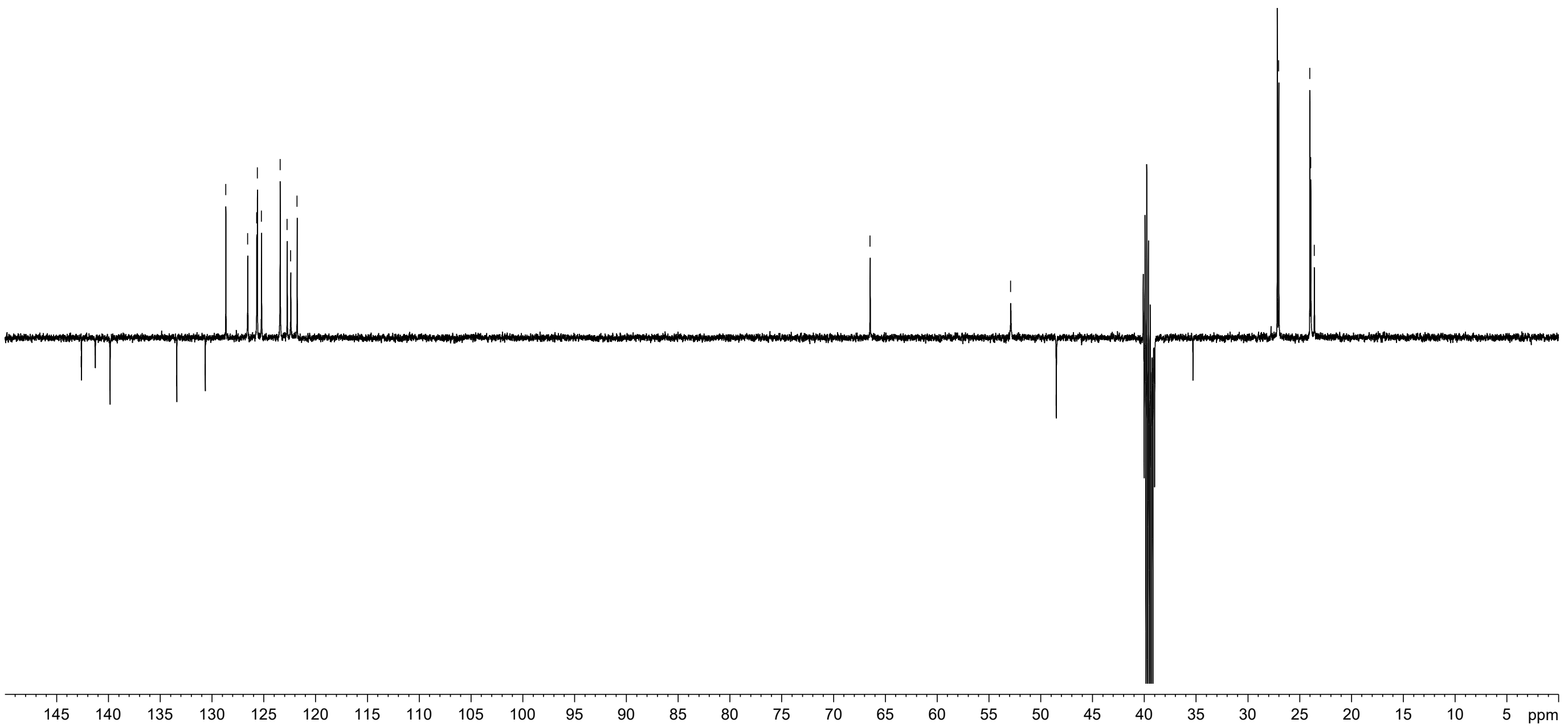


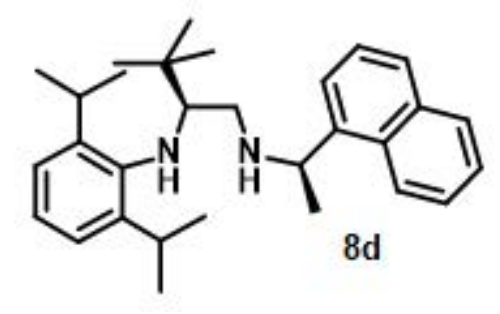

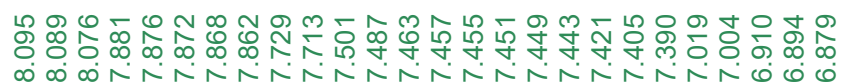



$\ldots$ tो

\begin{tabular}{|c|c|c|c|c|}
\hline 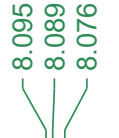 & 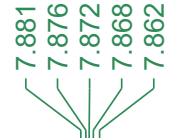 & iㅏㅅ & 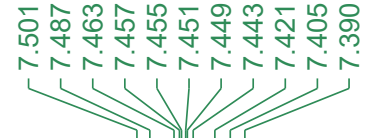 & í \\
\hline
\end{tabular}
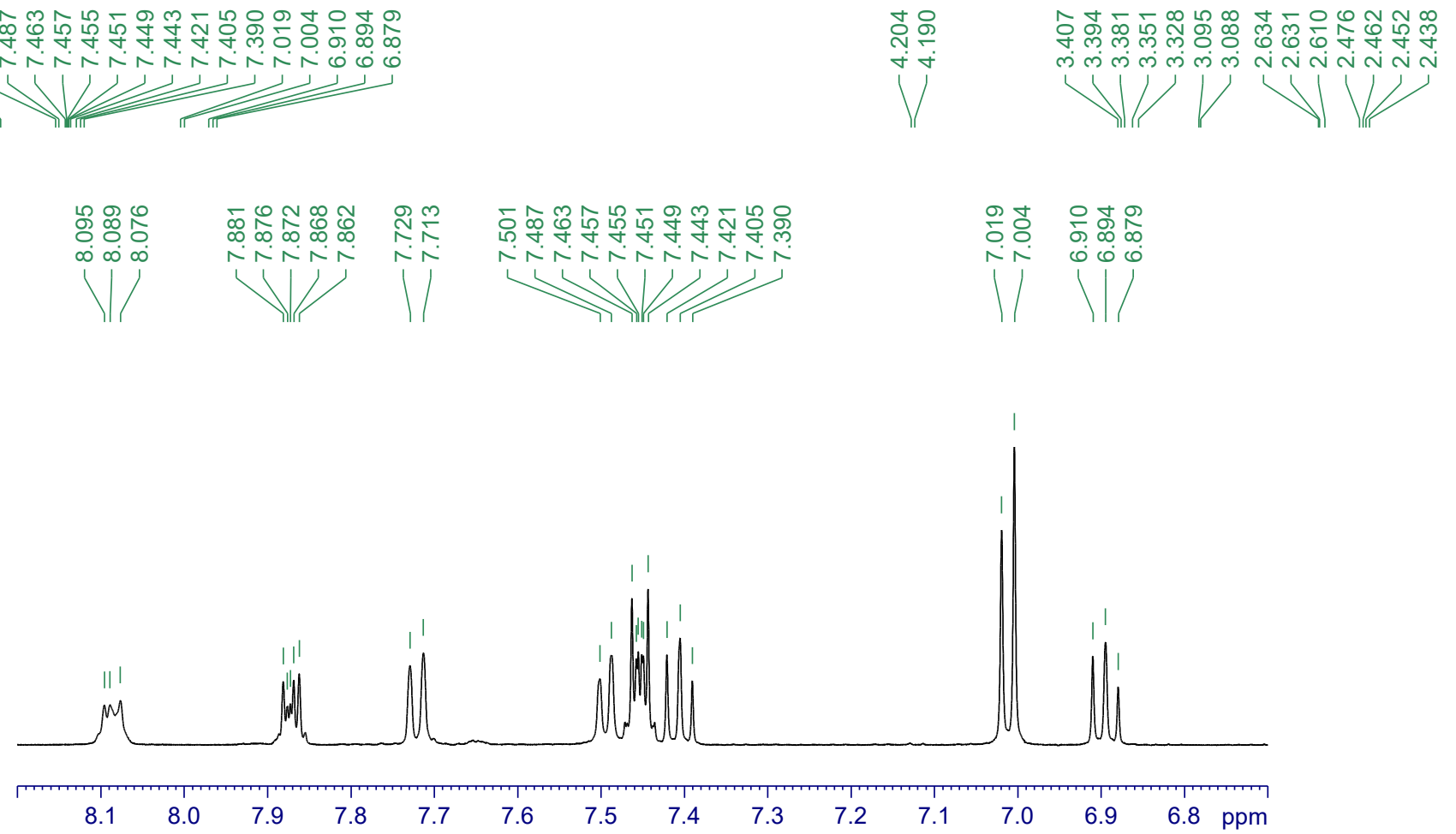

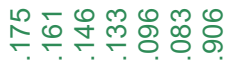
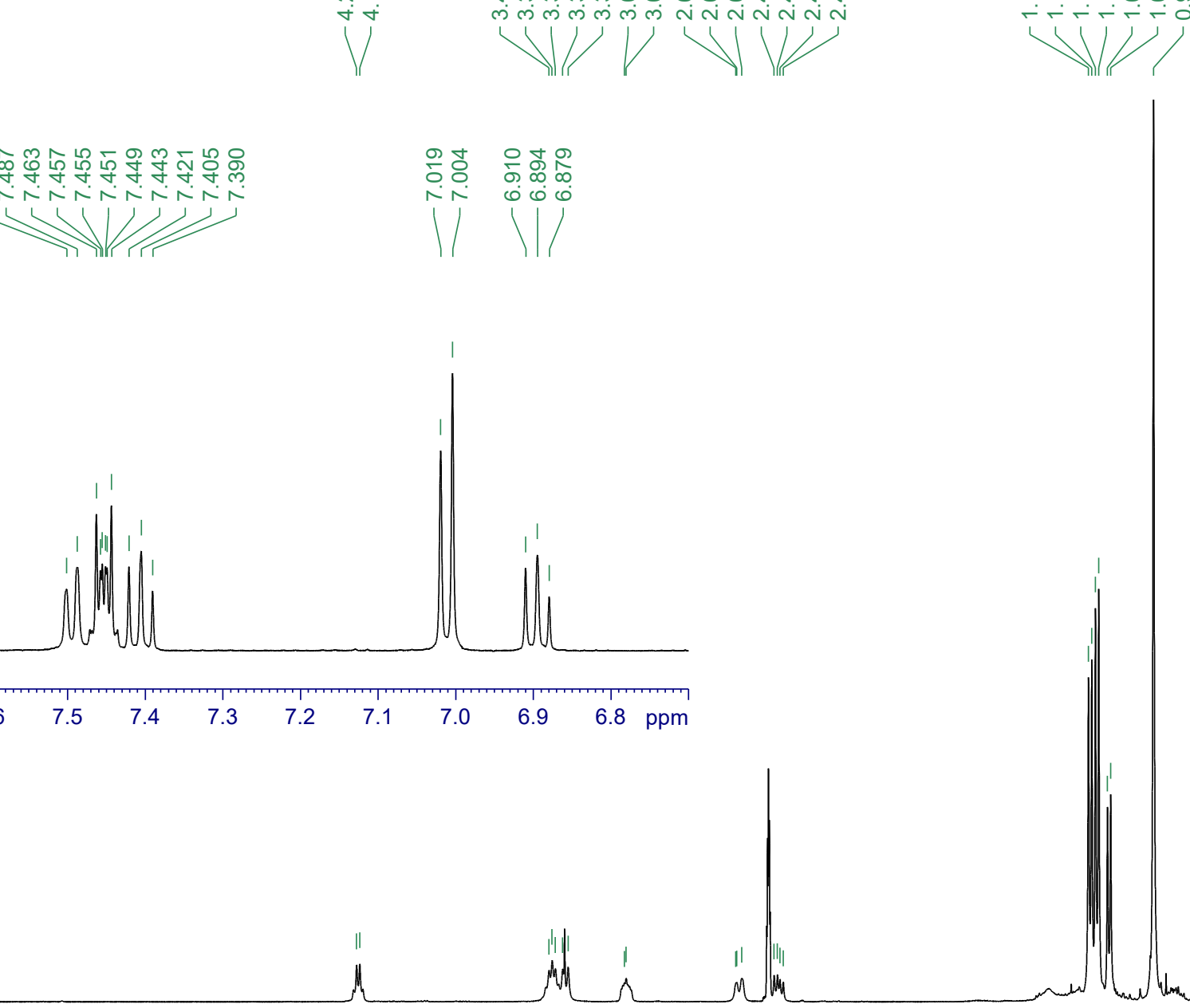


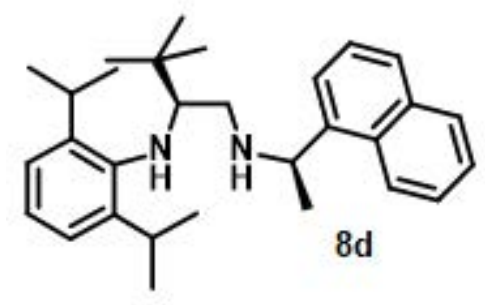

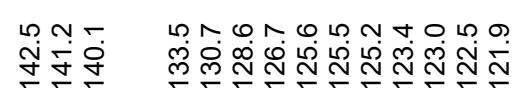

HI WNWIII

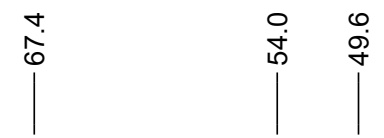

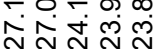

VV

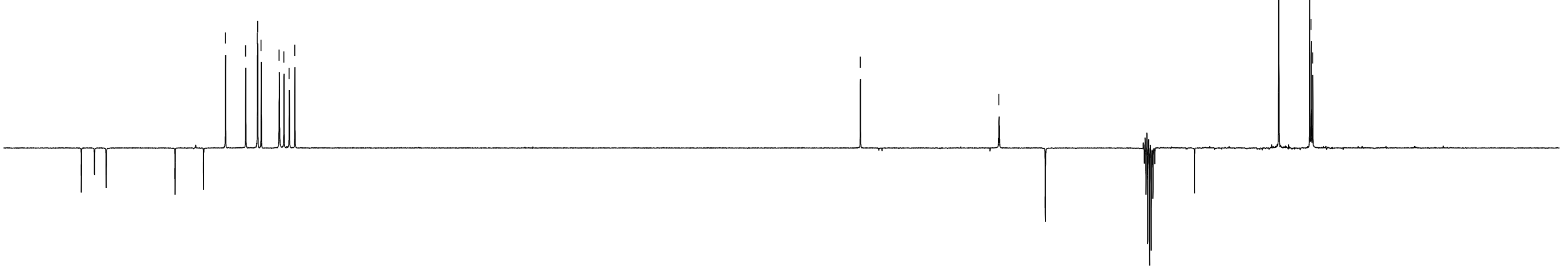



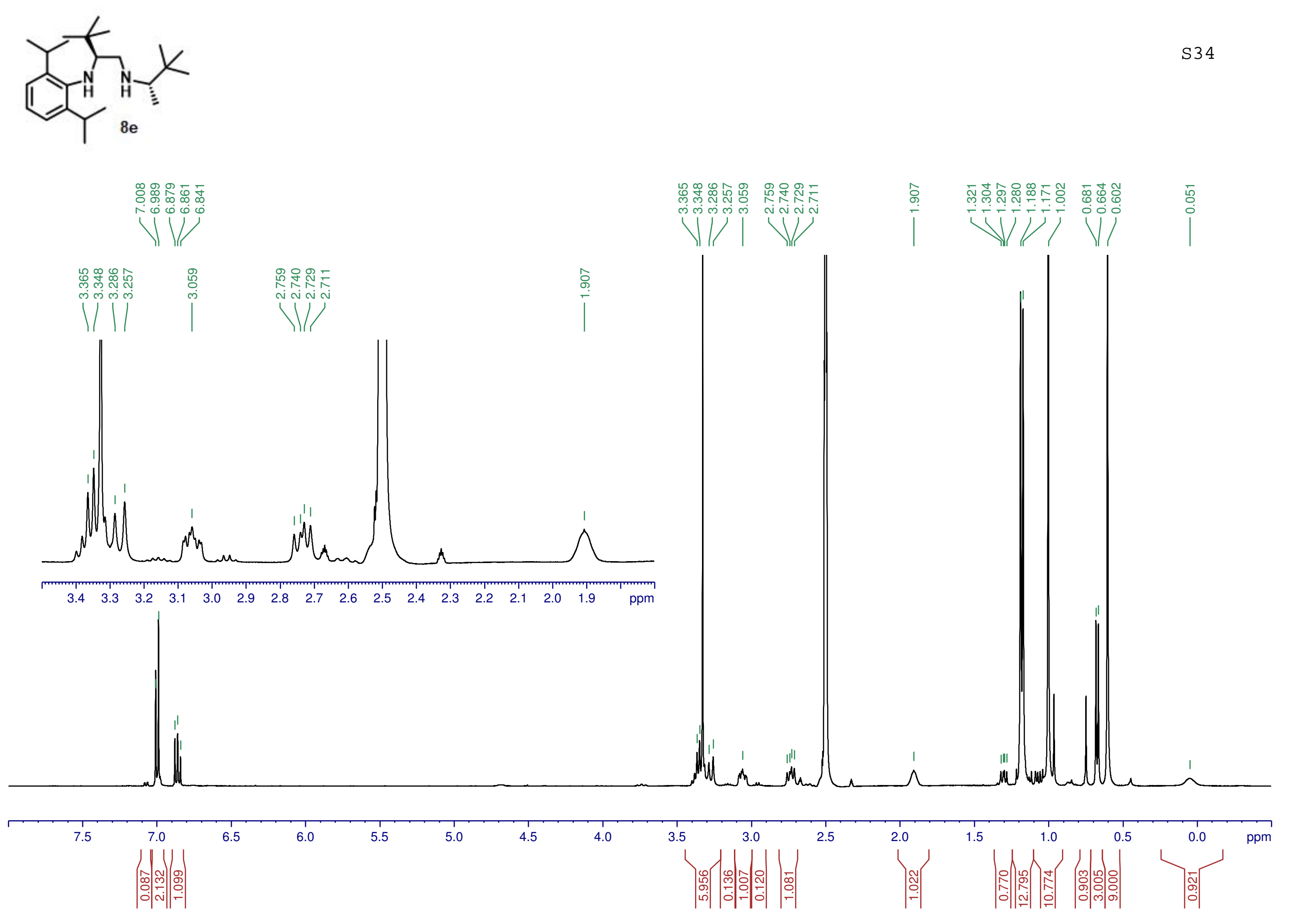

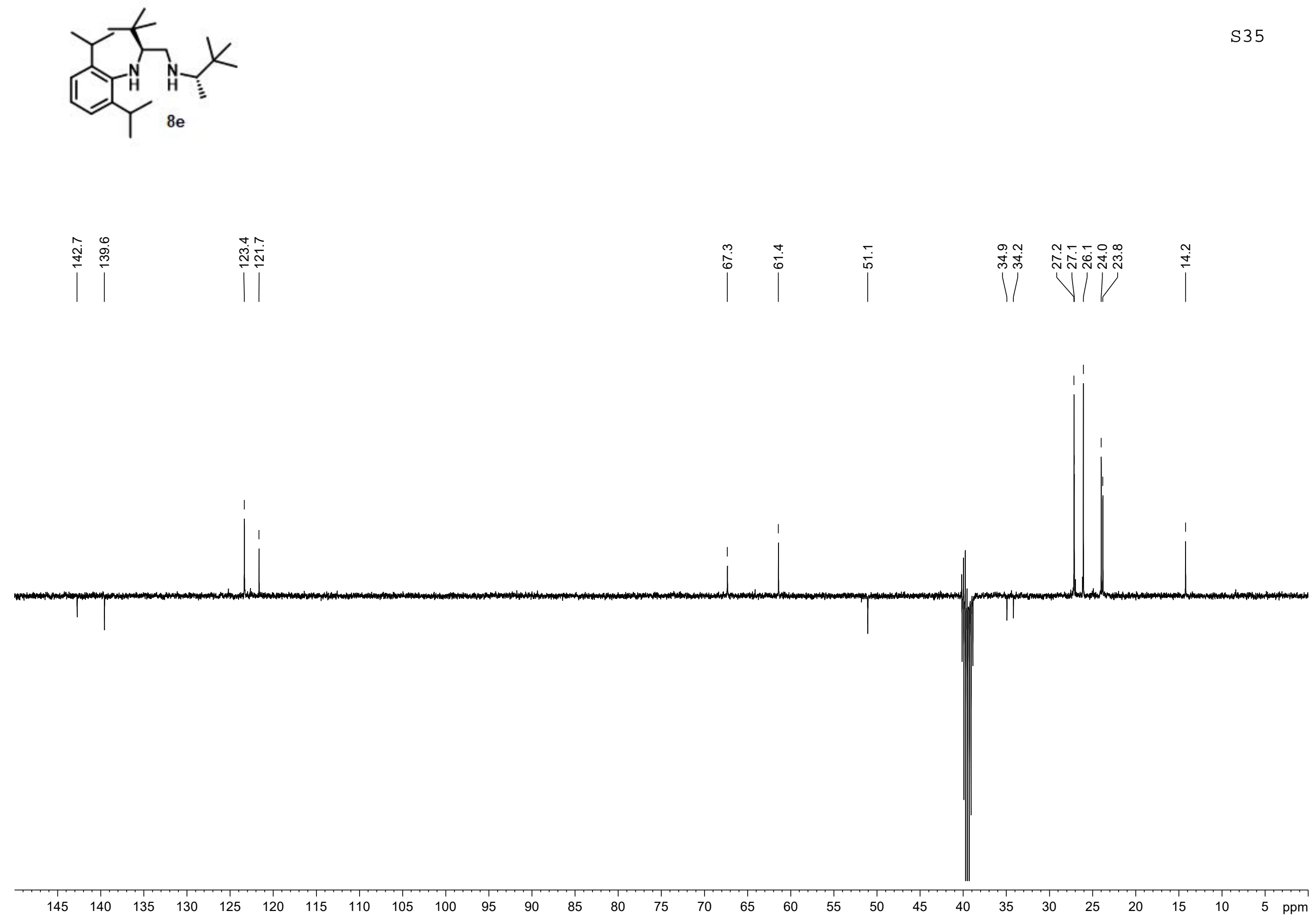



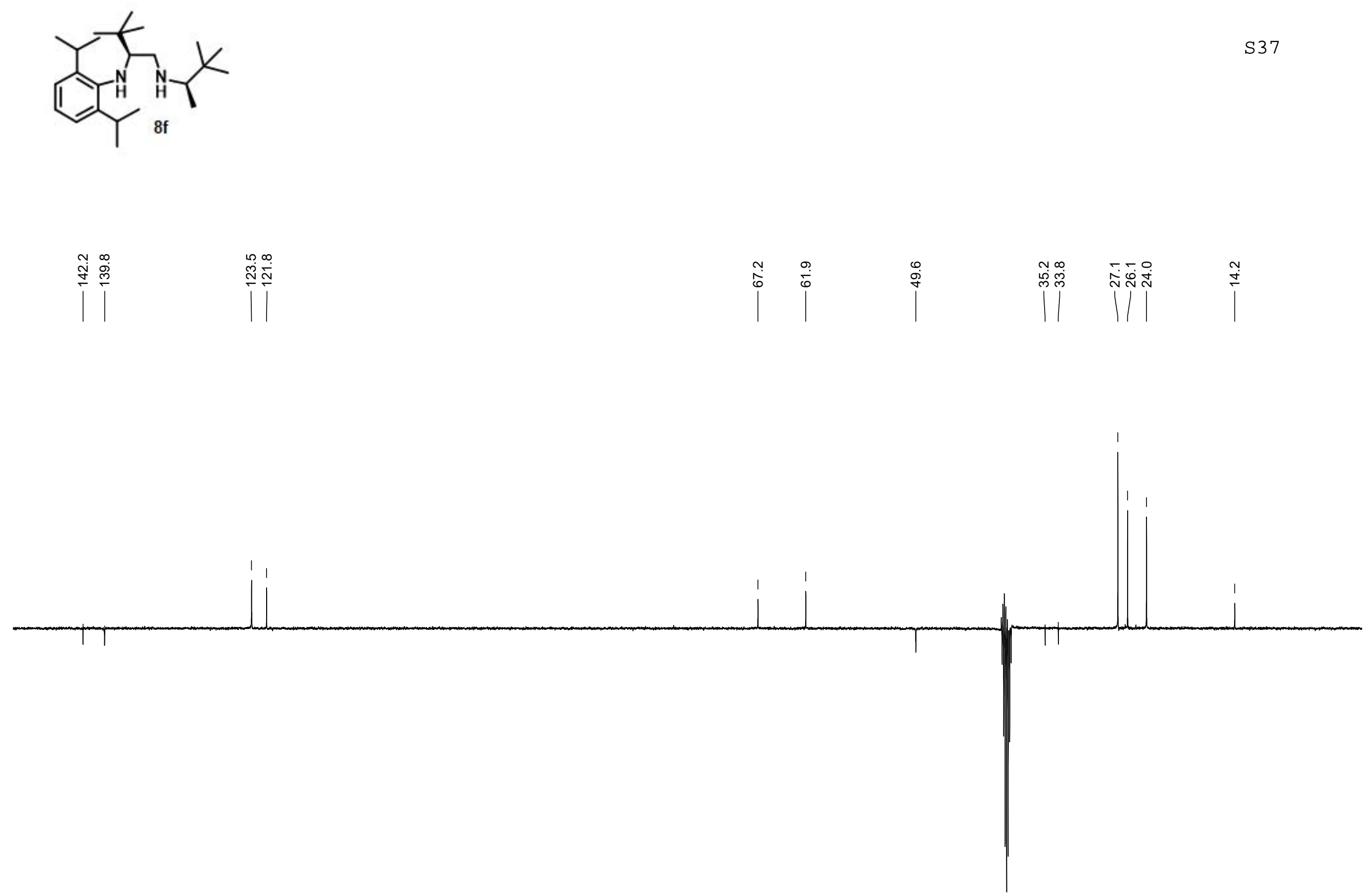

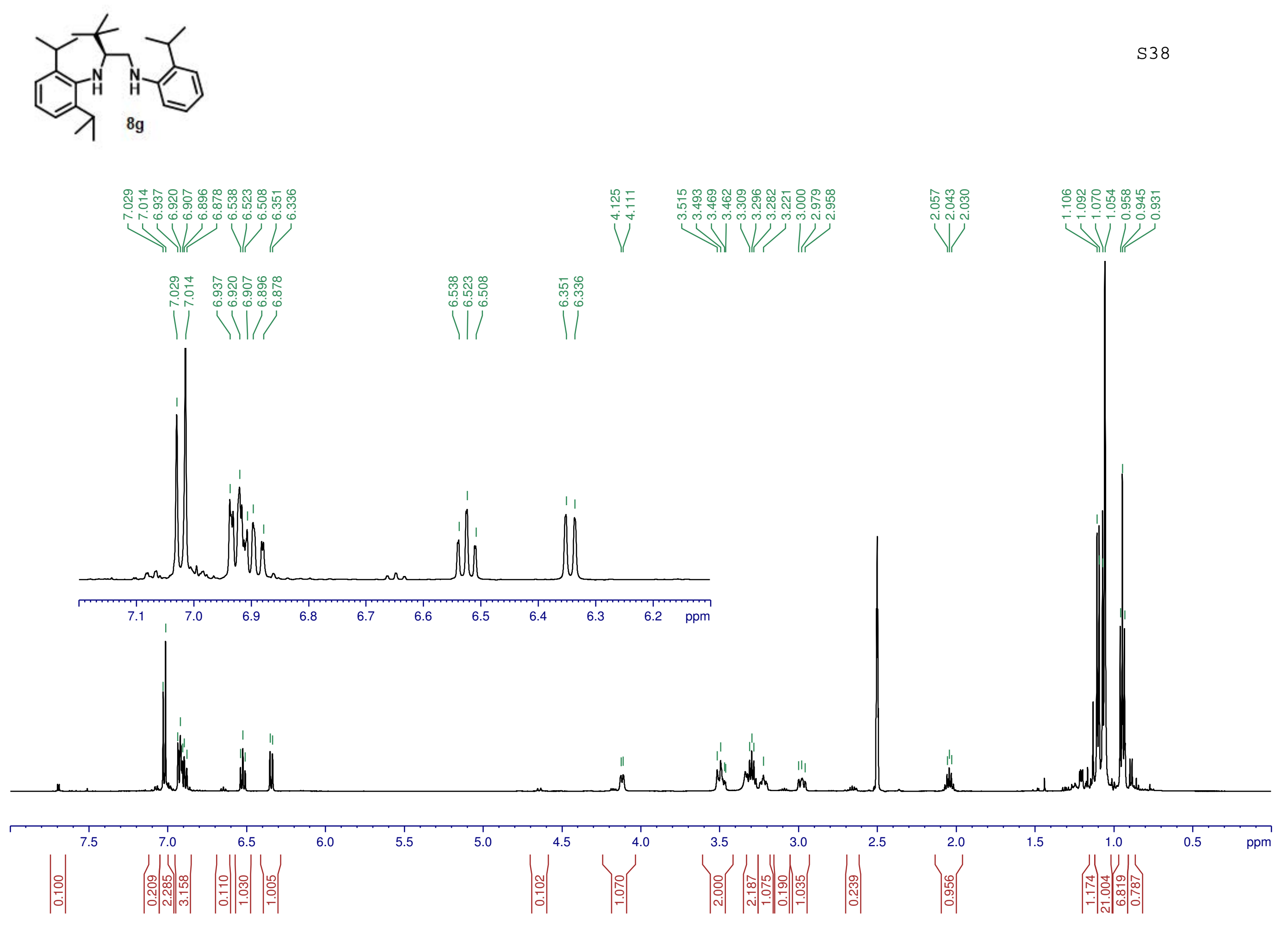



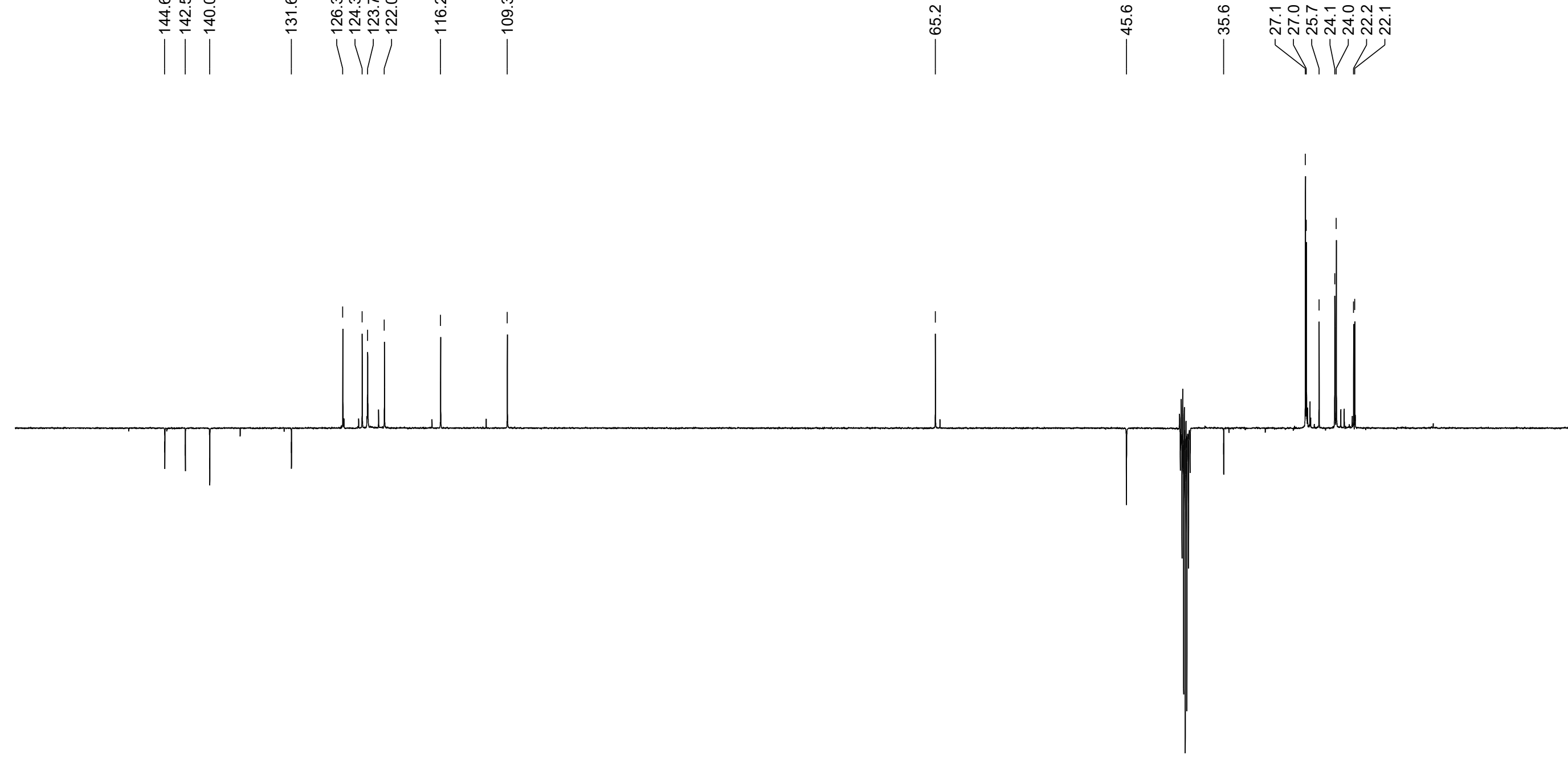


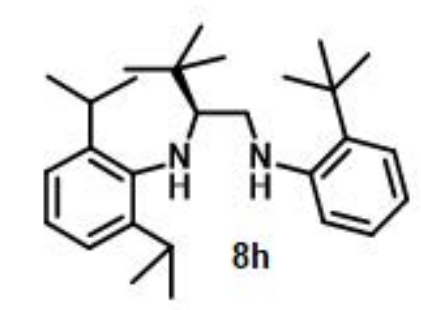

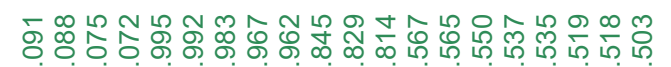

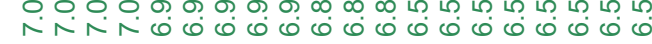

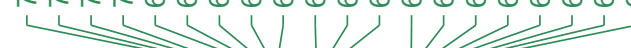

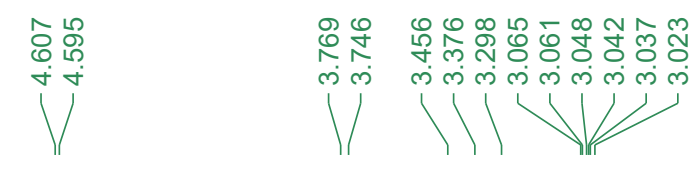

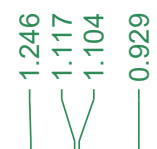
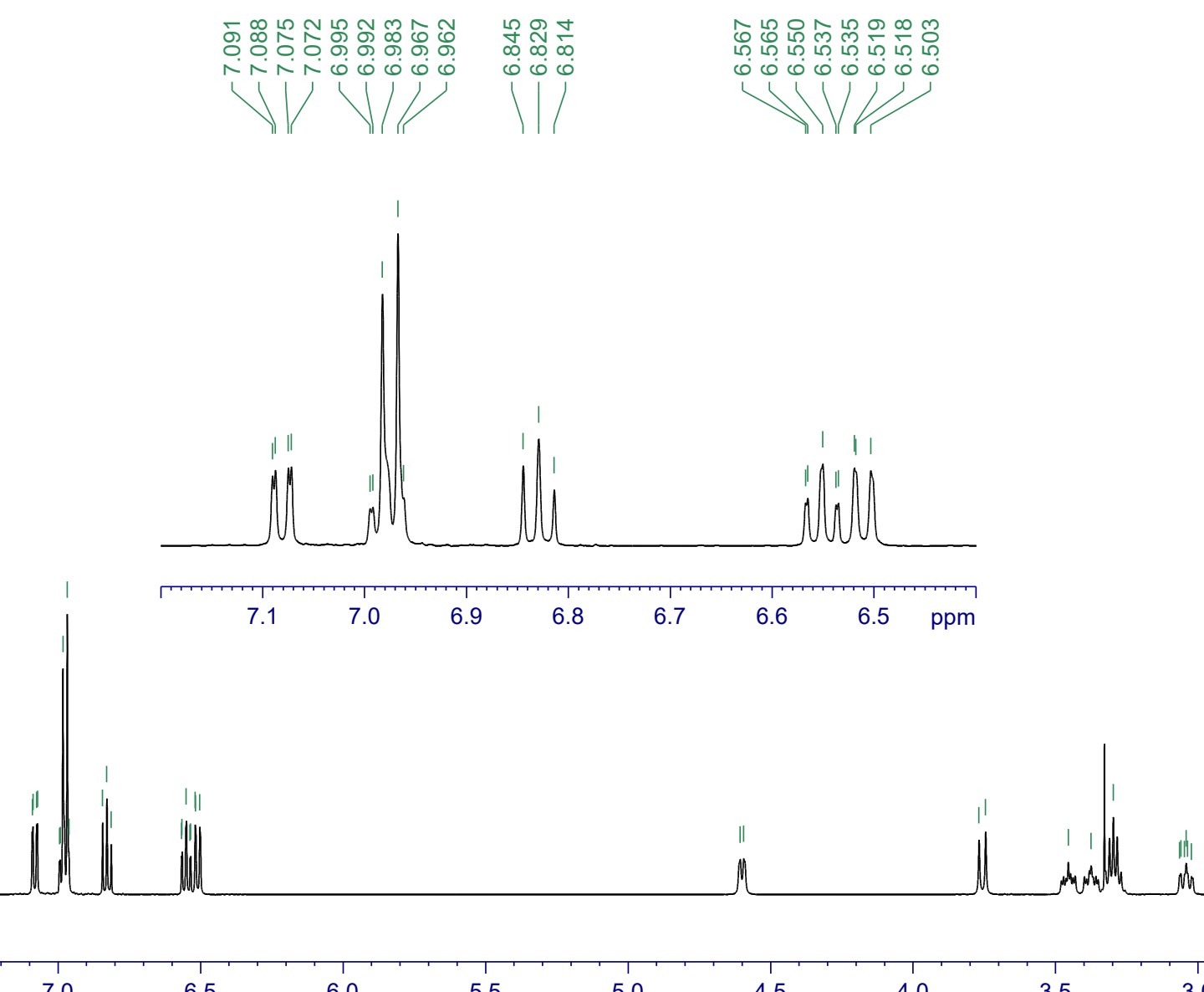

5.5

5.0

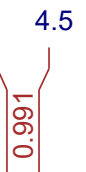

4.0

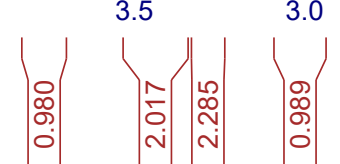

2.0

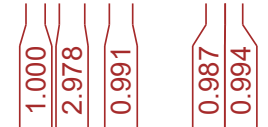




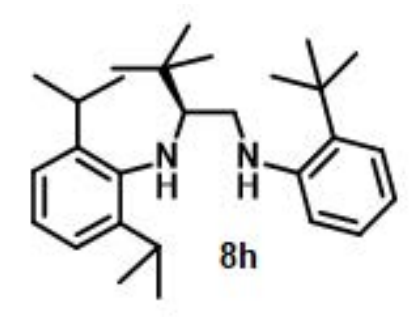

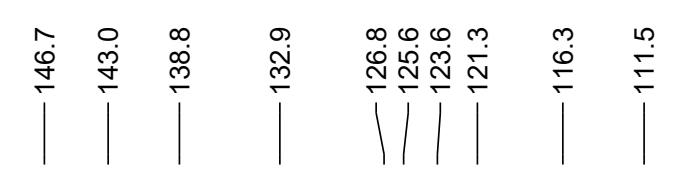
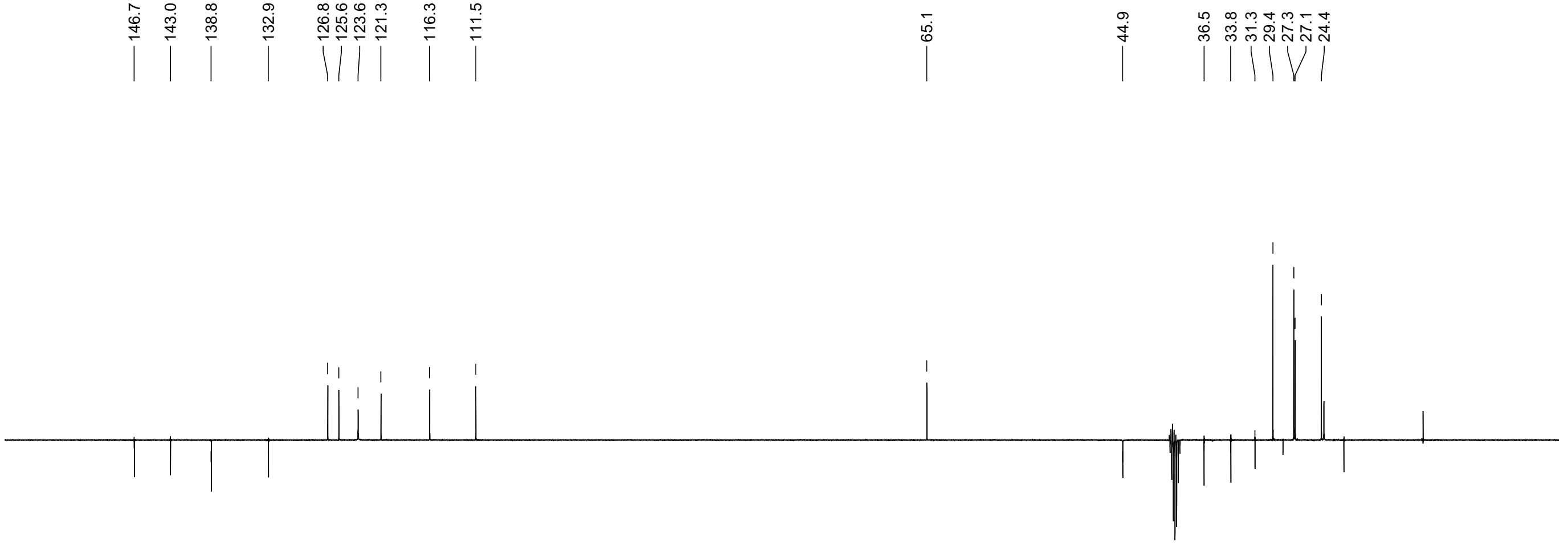

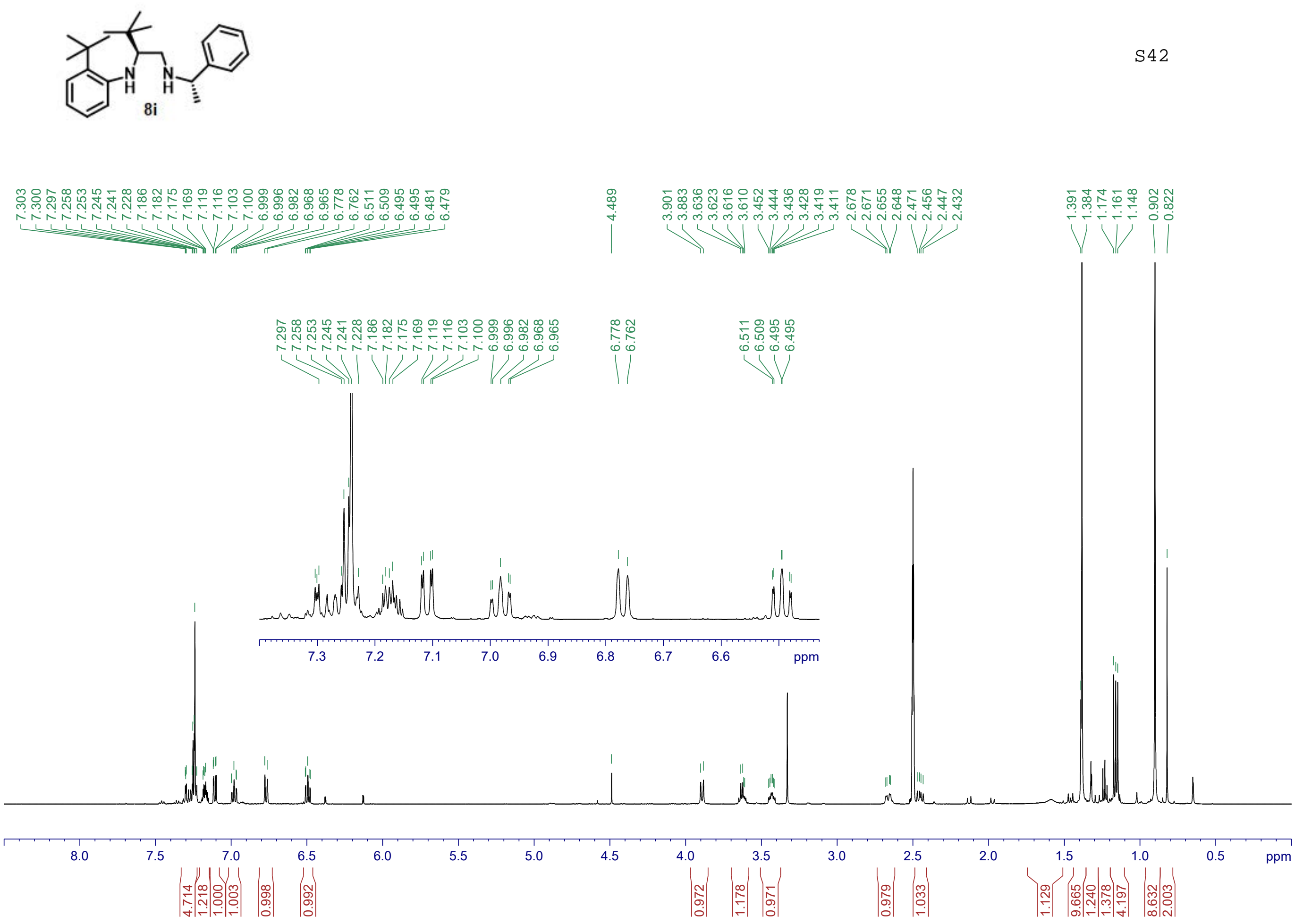


$\left.\right|^{0}$
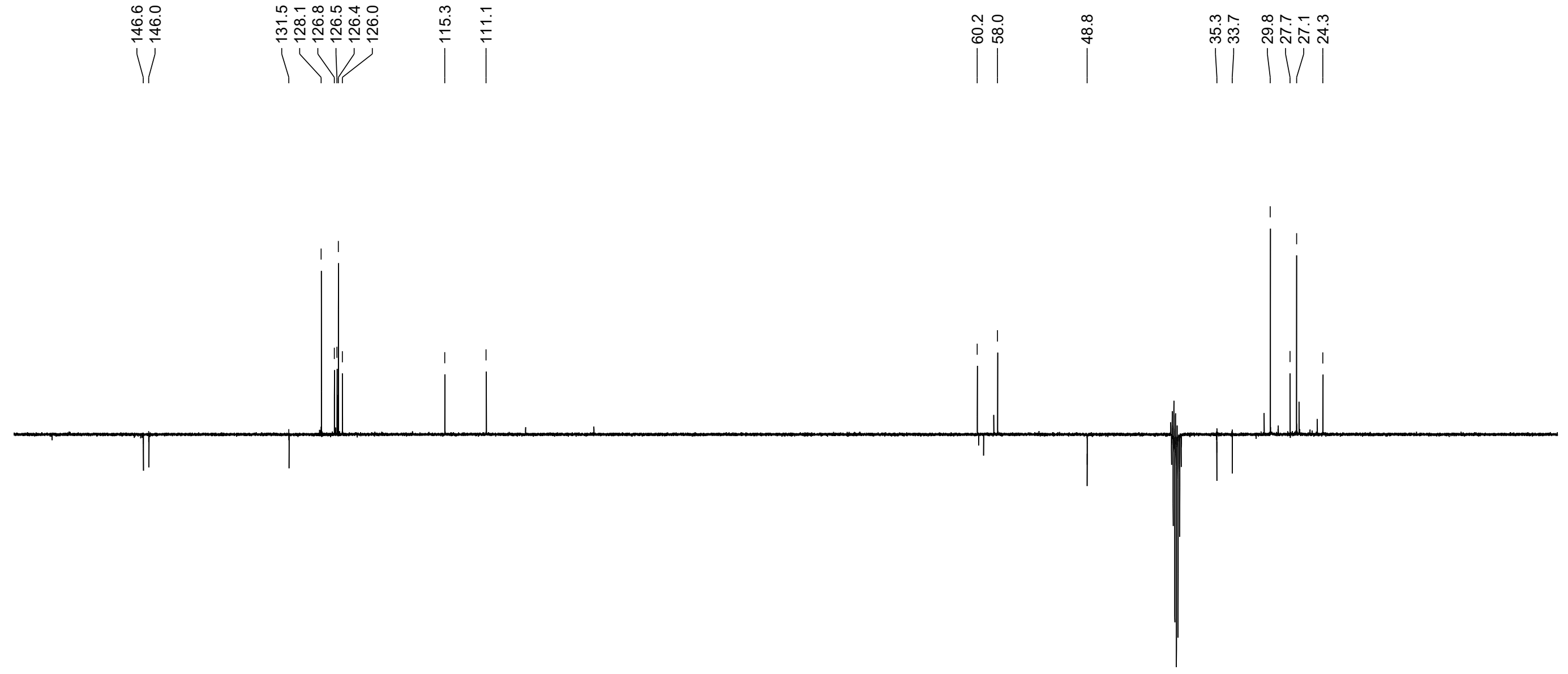

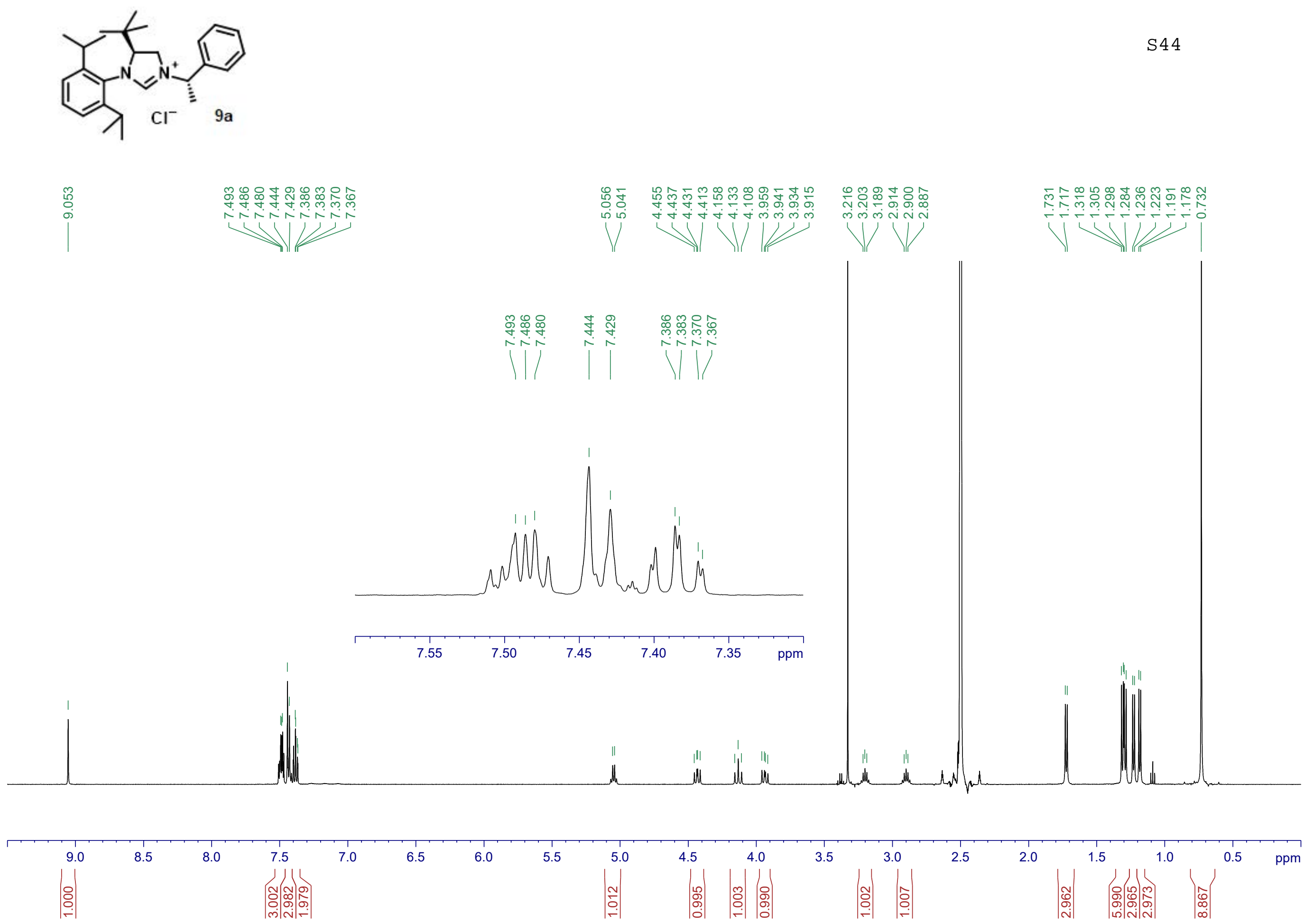

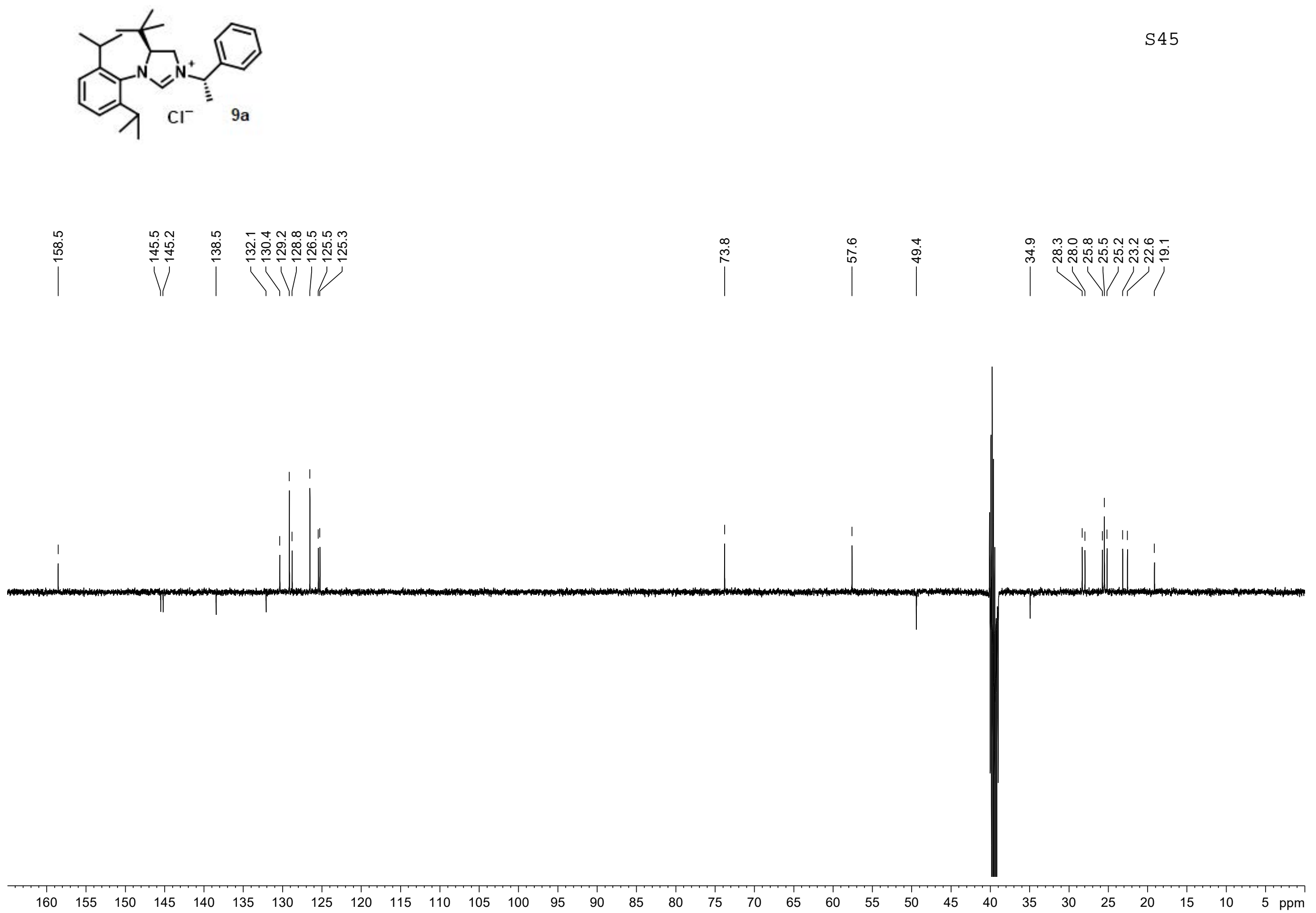

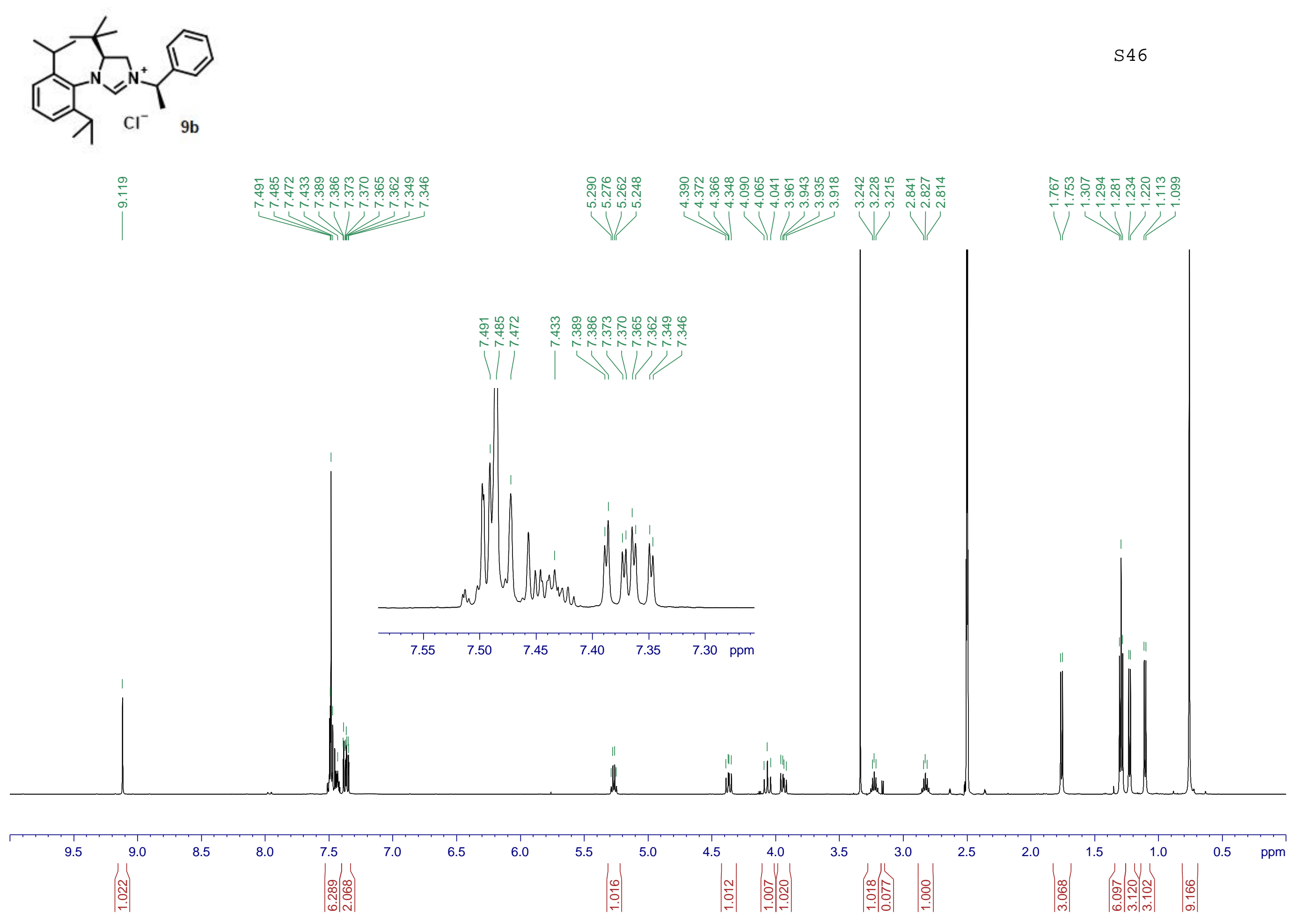

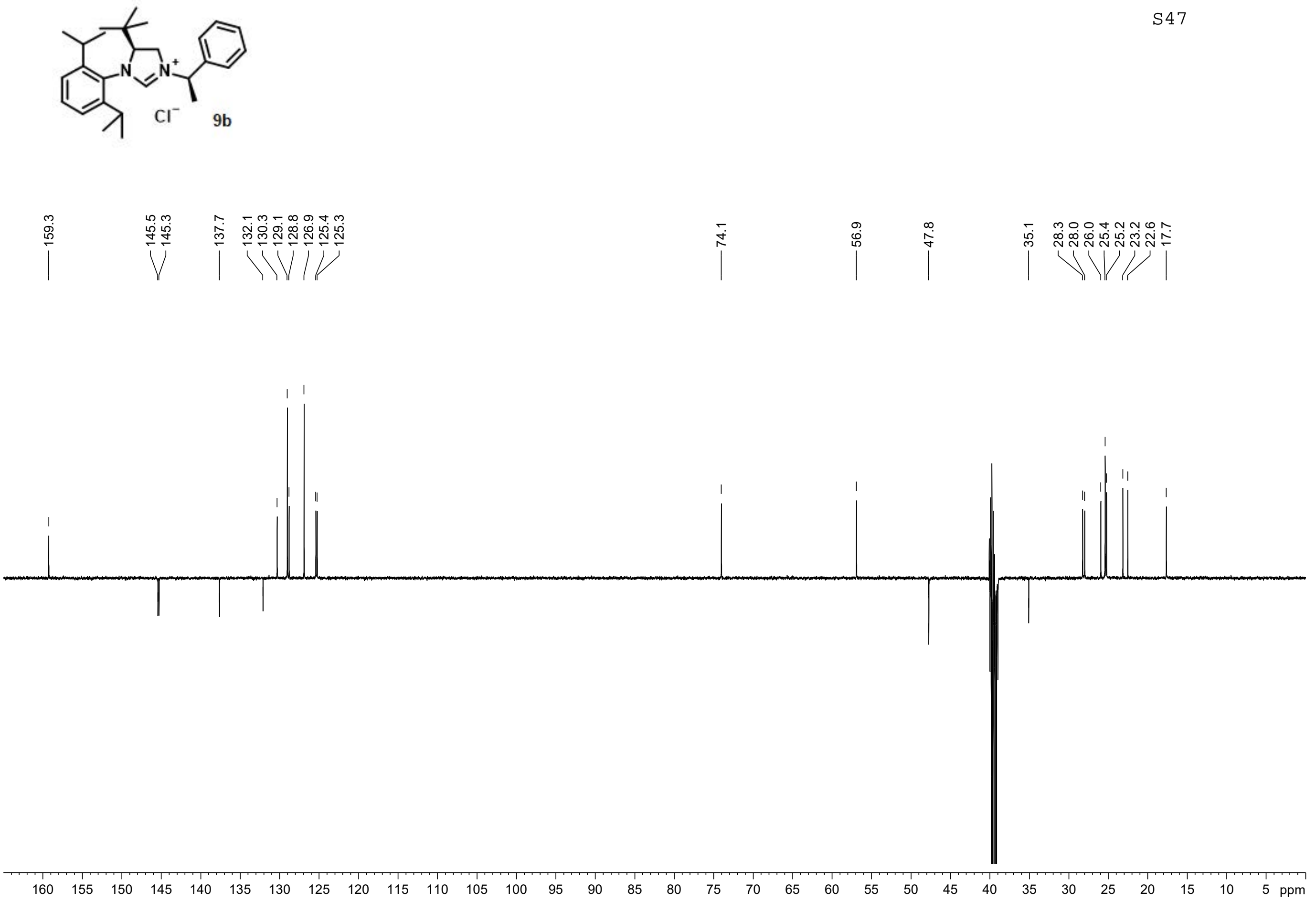

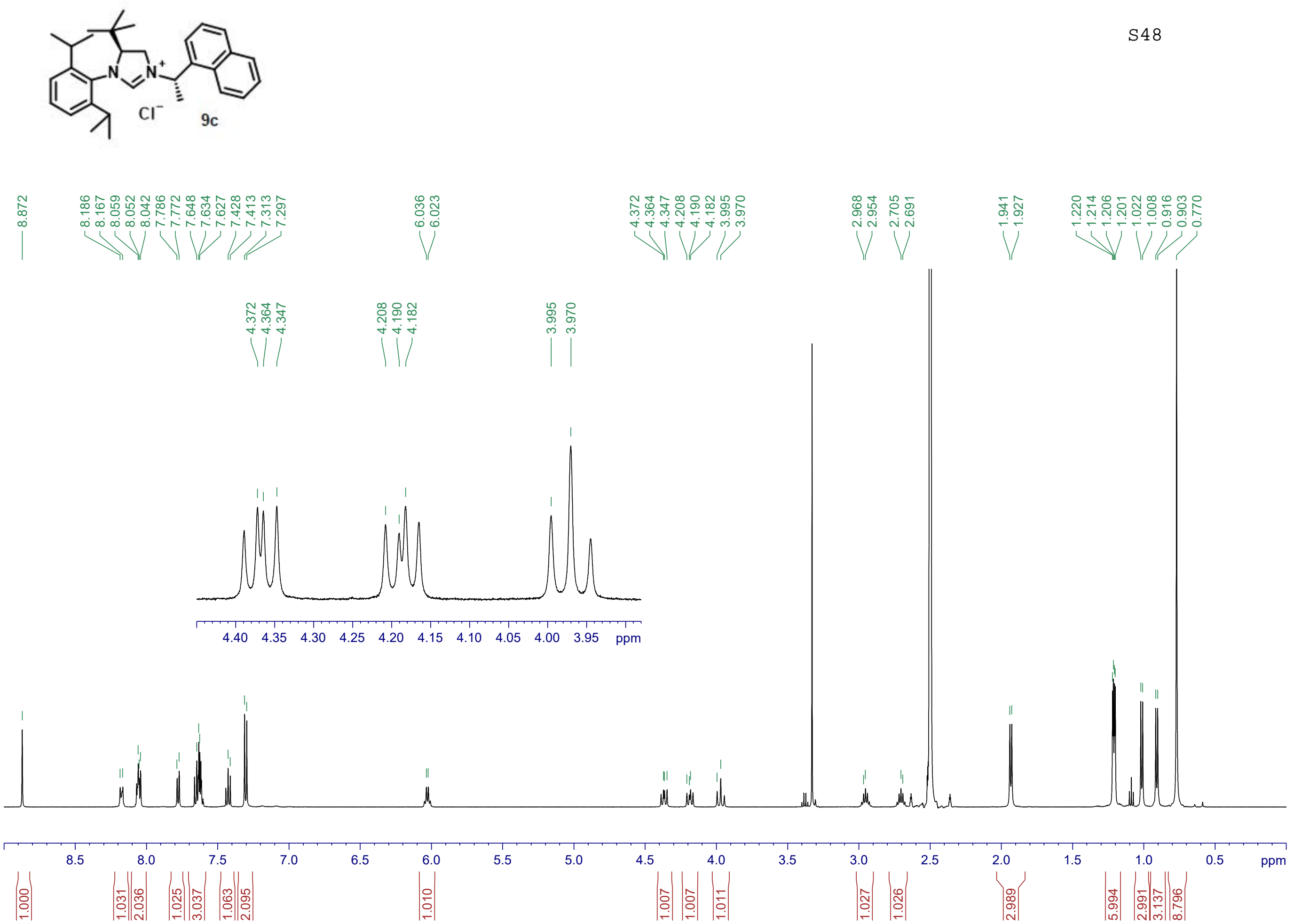

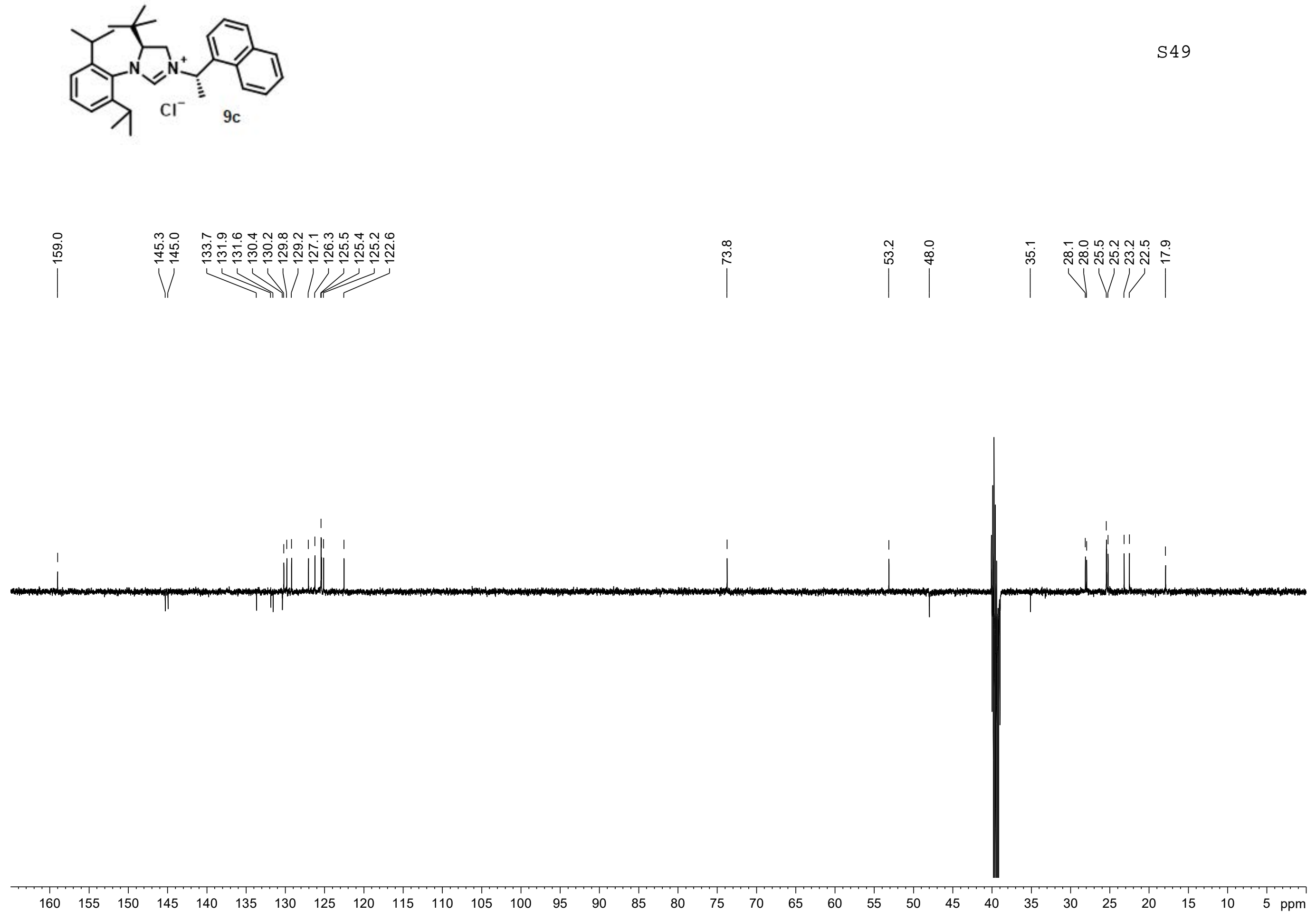

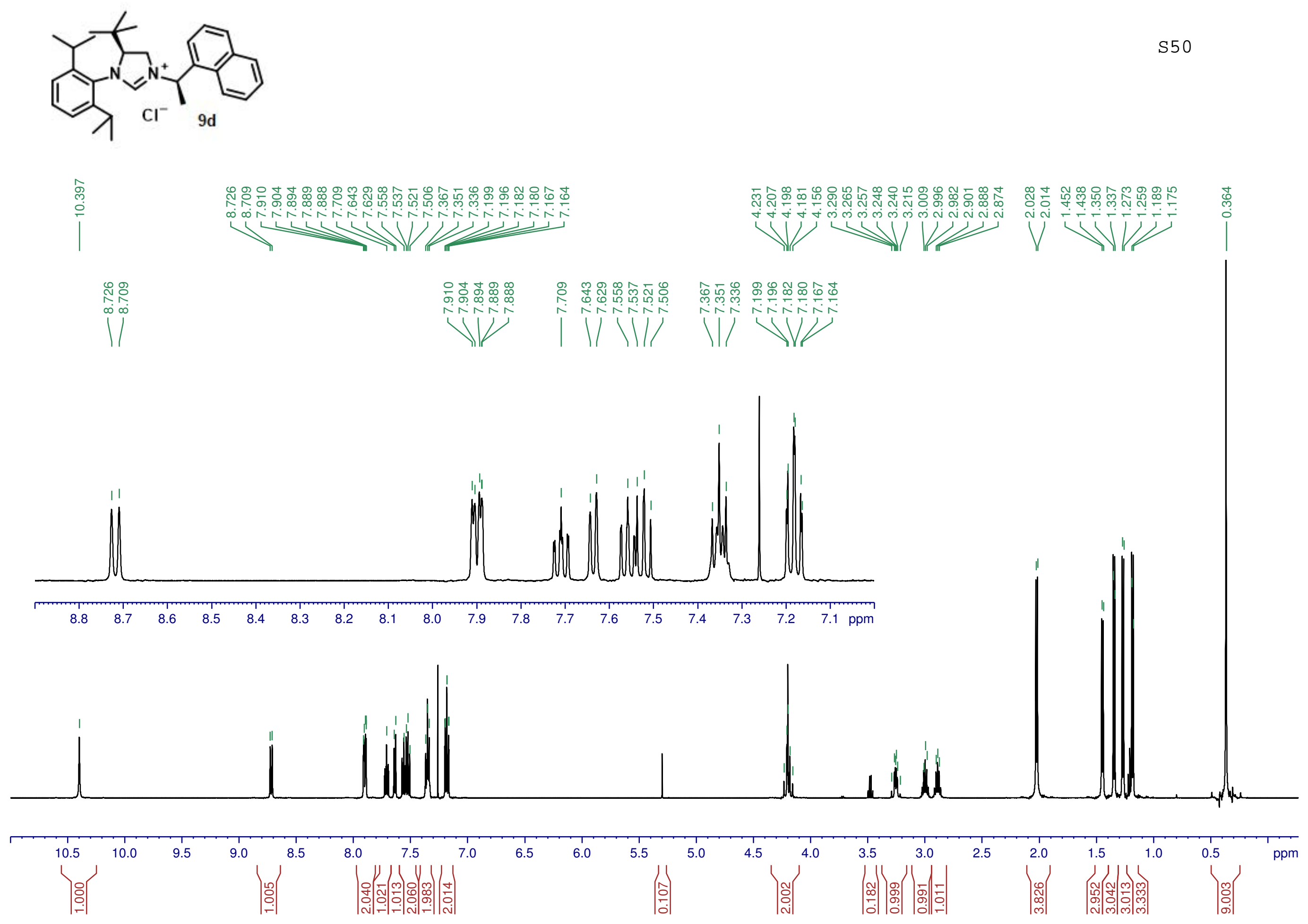

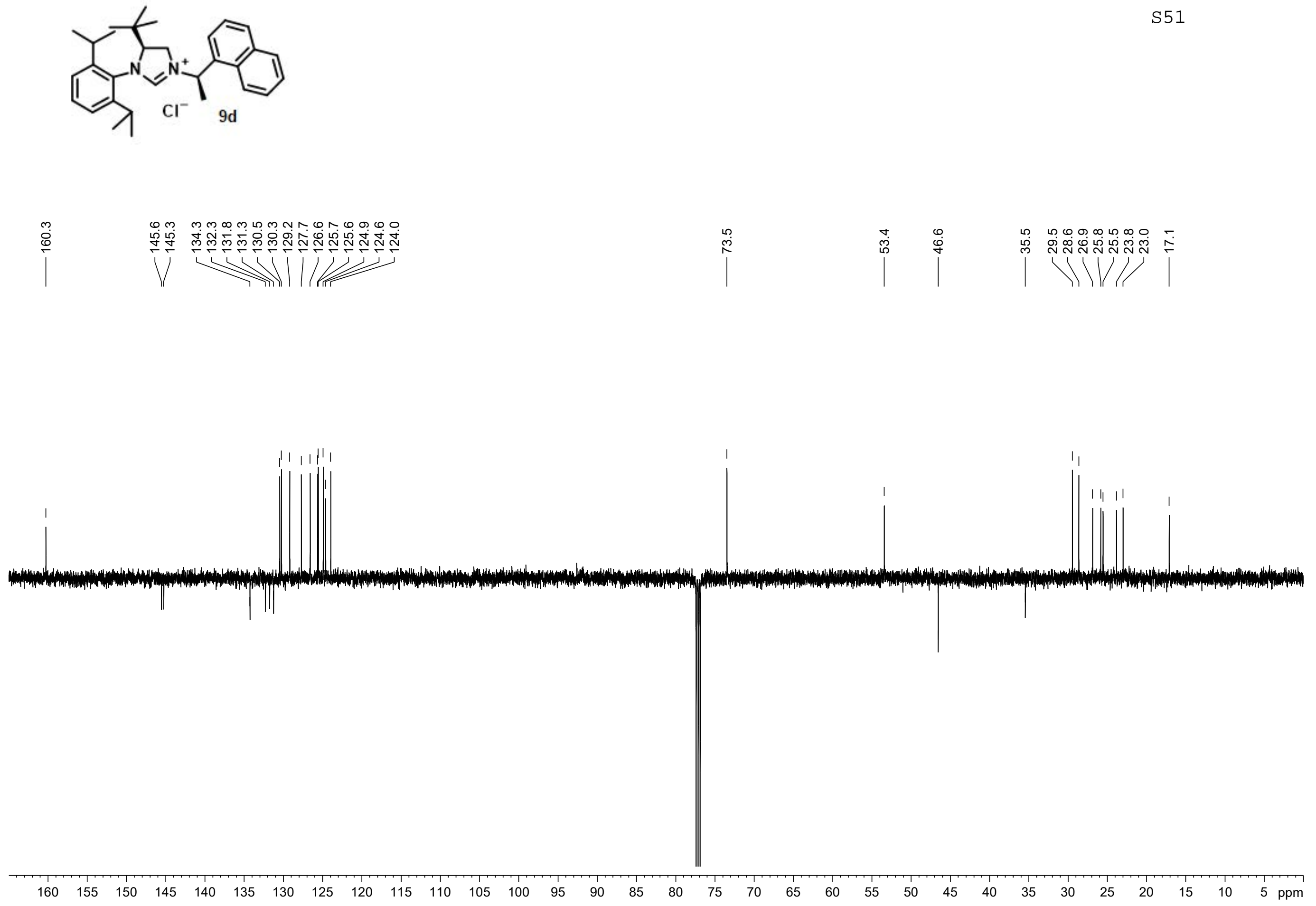

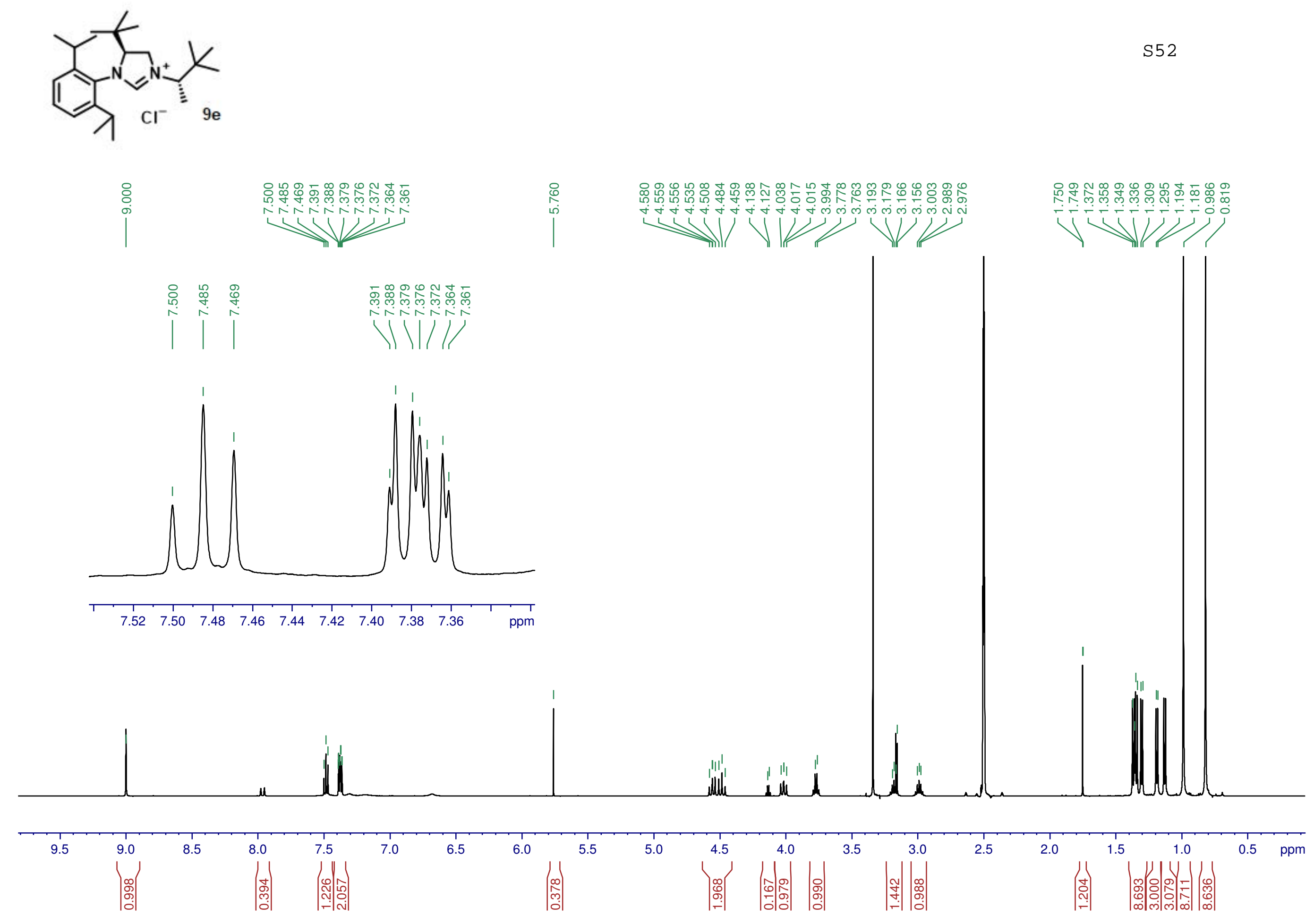

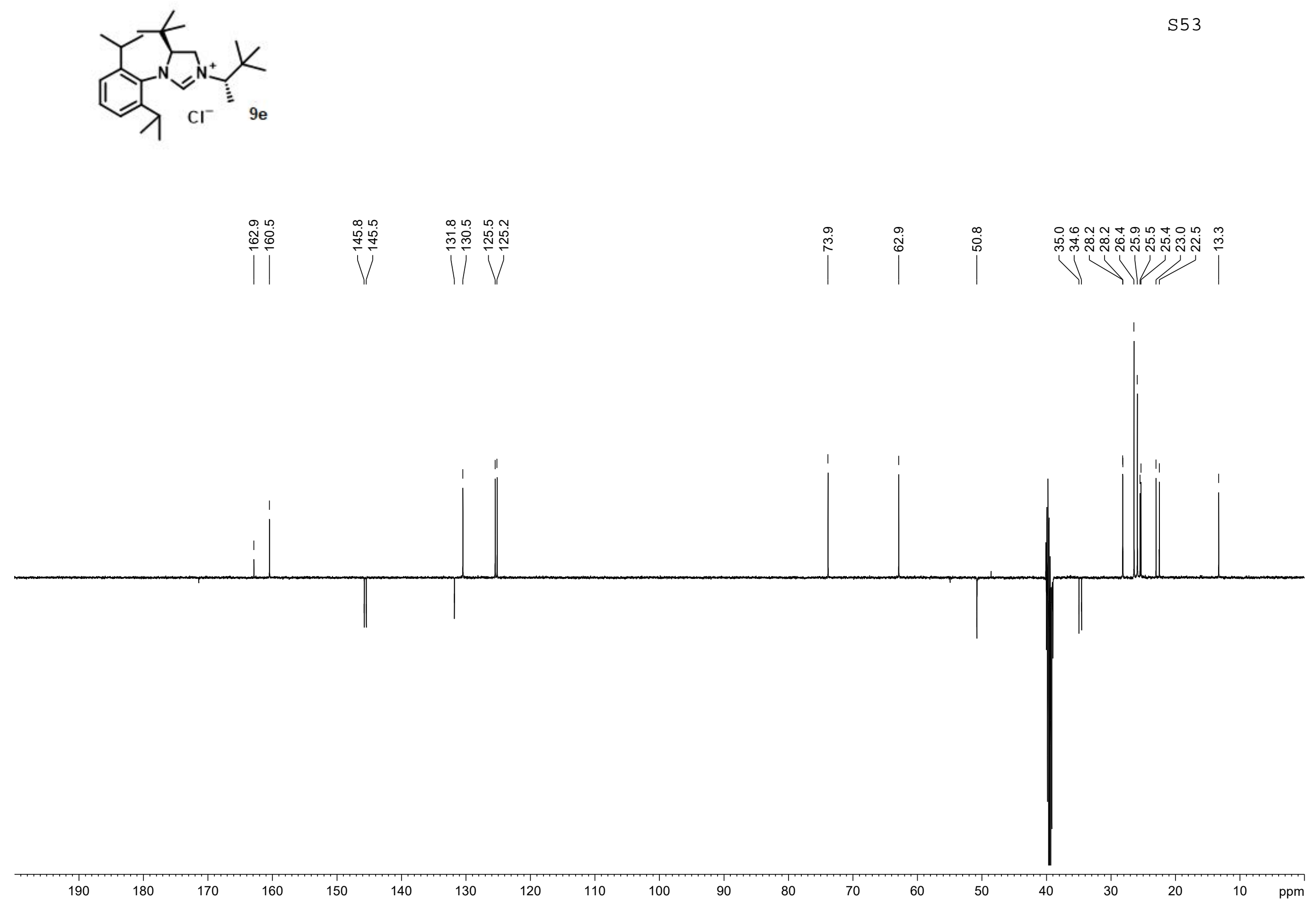


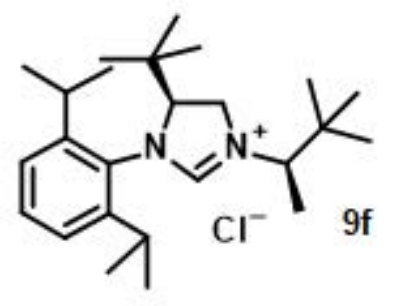

$\left.\right|_{0}$
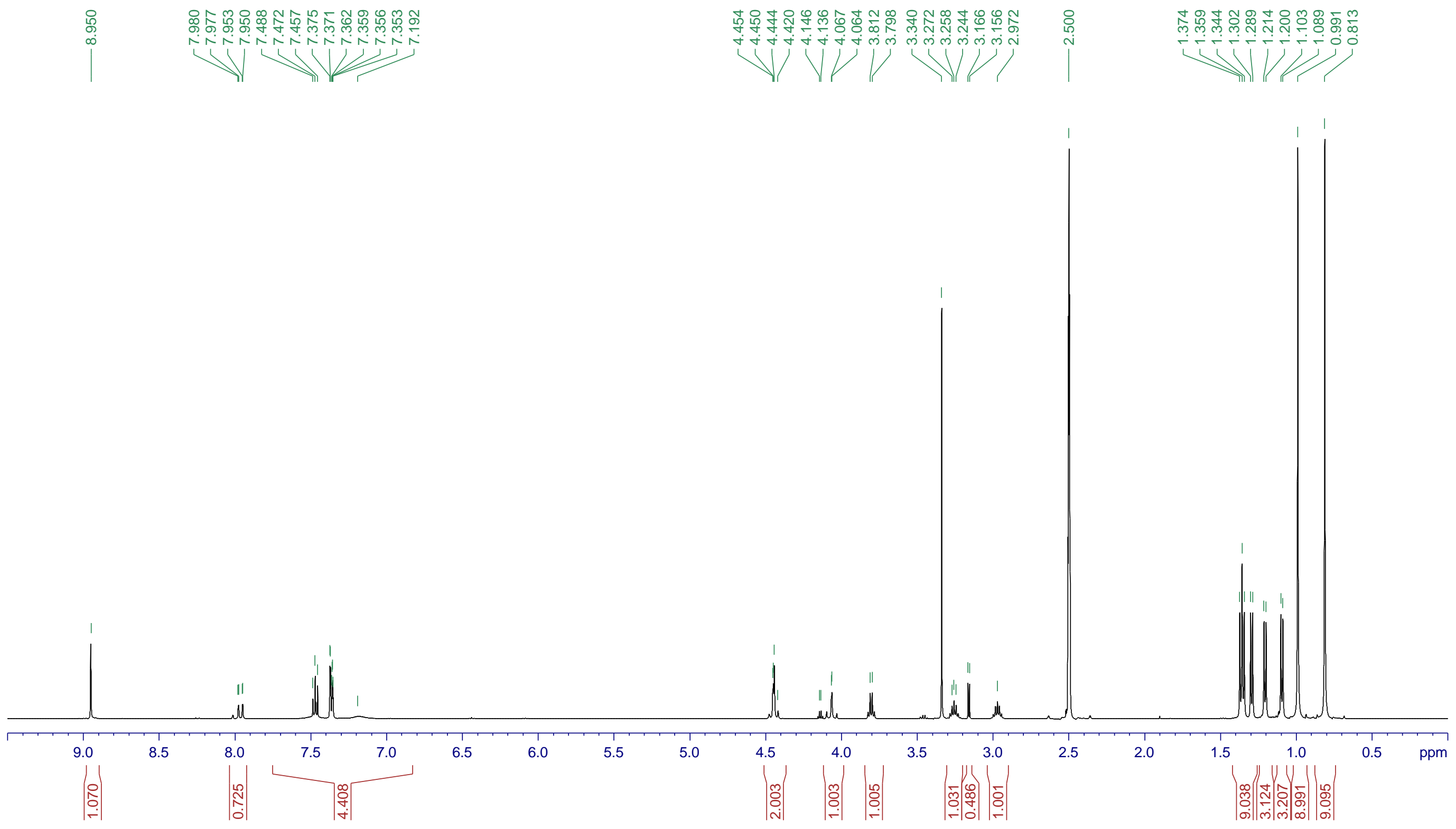

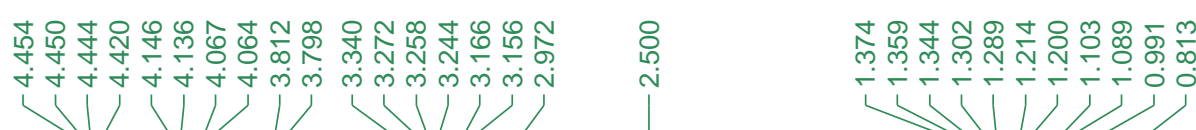
(1)

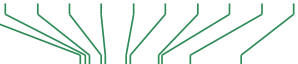



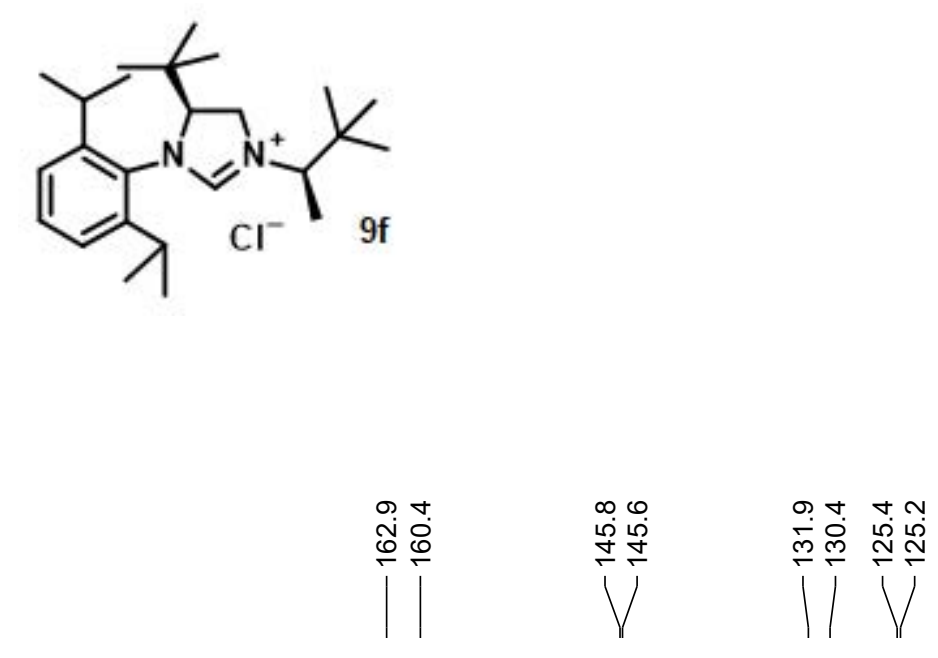

$$
\overbrace{}^{\dot{5}}
$$

ஷீ

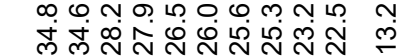

111

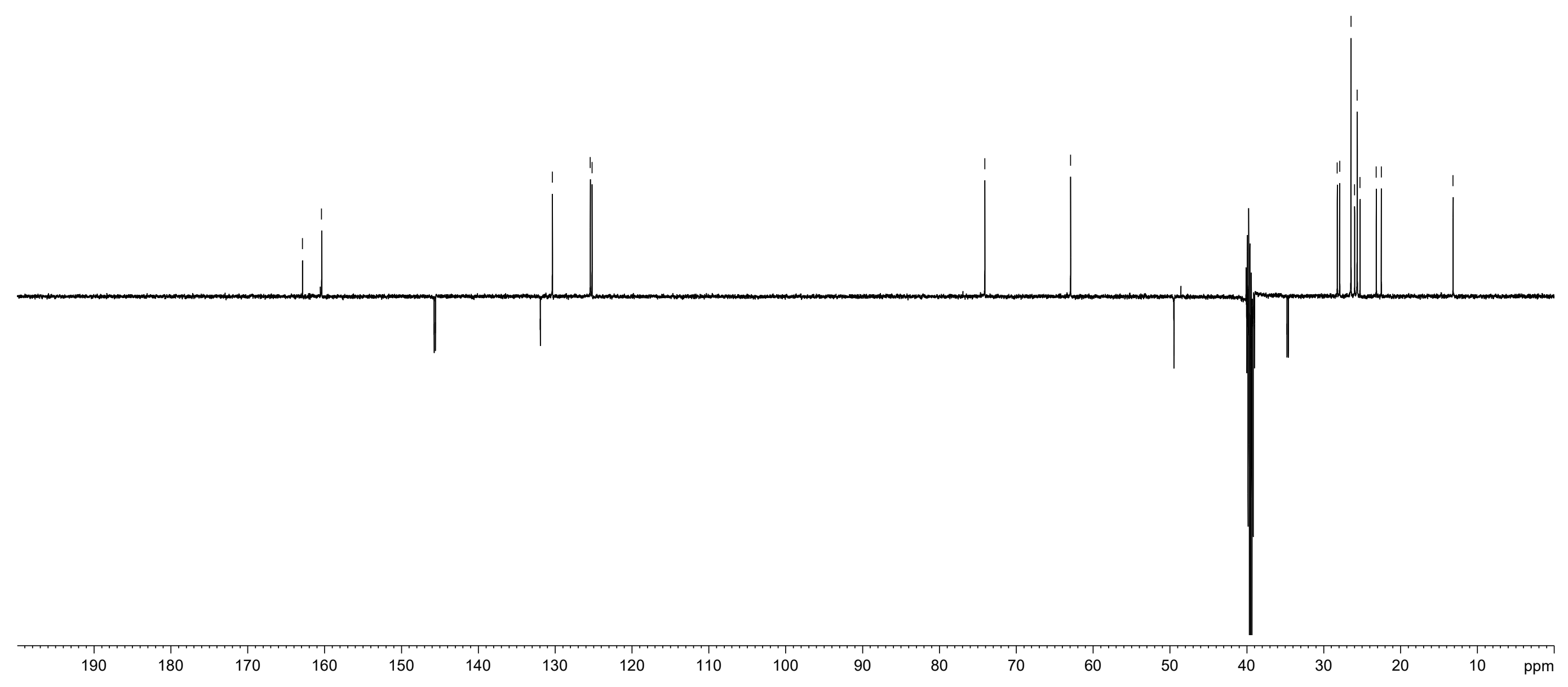



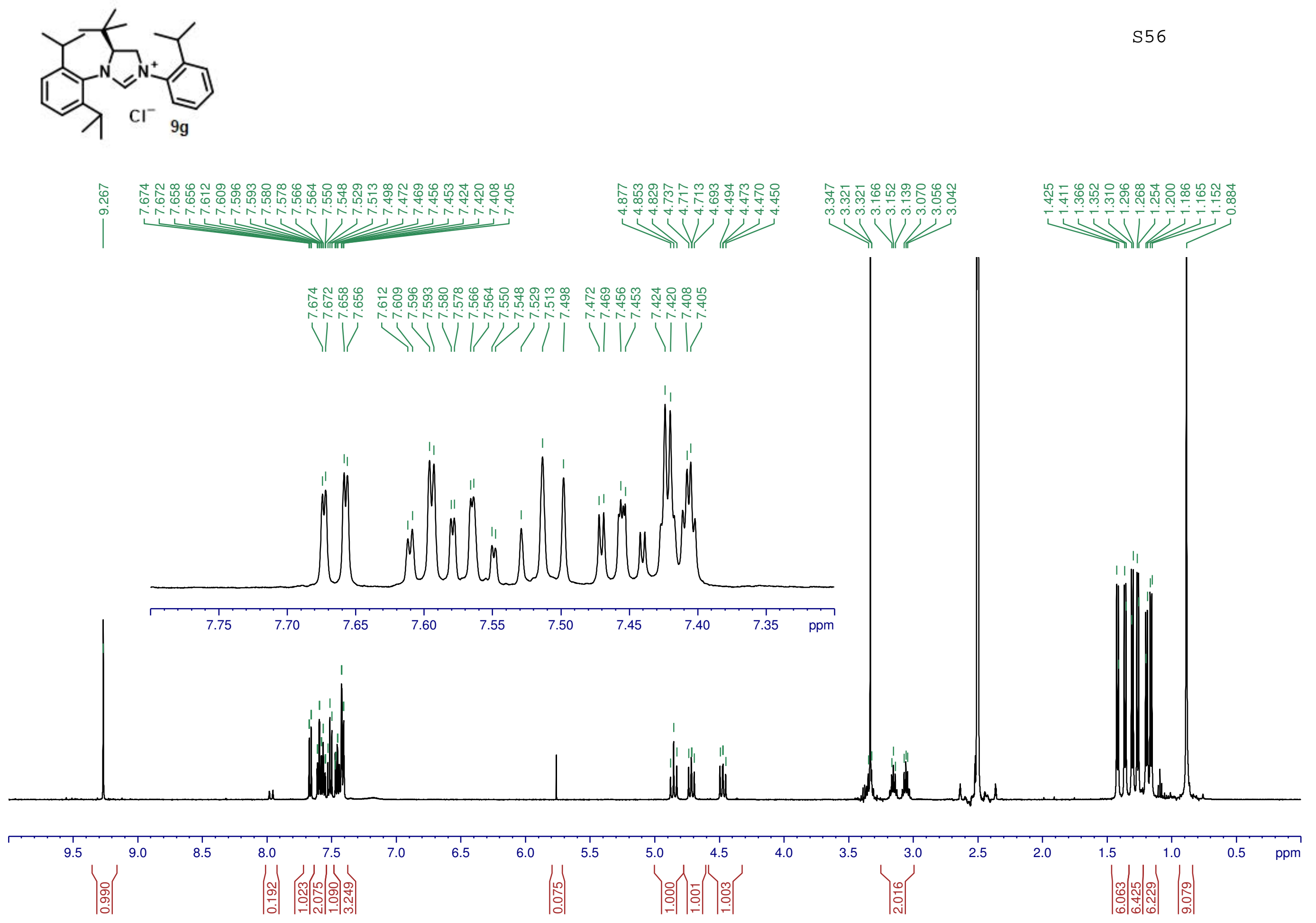


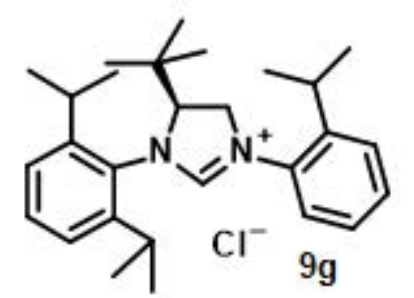

$\overbrace{0}^{\infty} \quad \sum_{0}^{0}$

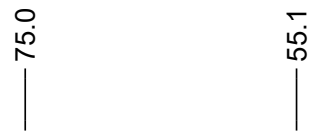

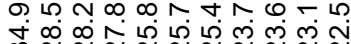

$\longrightarrow$

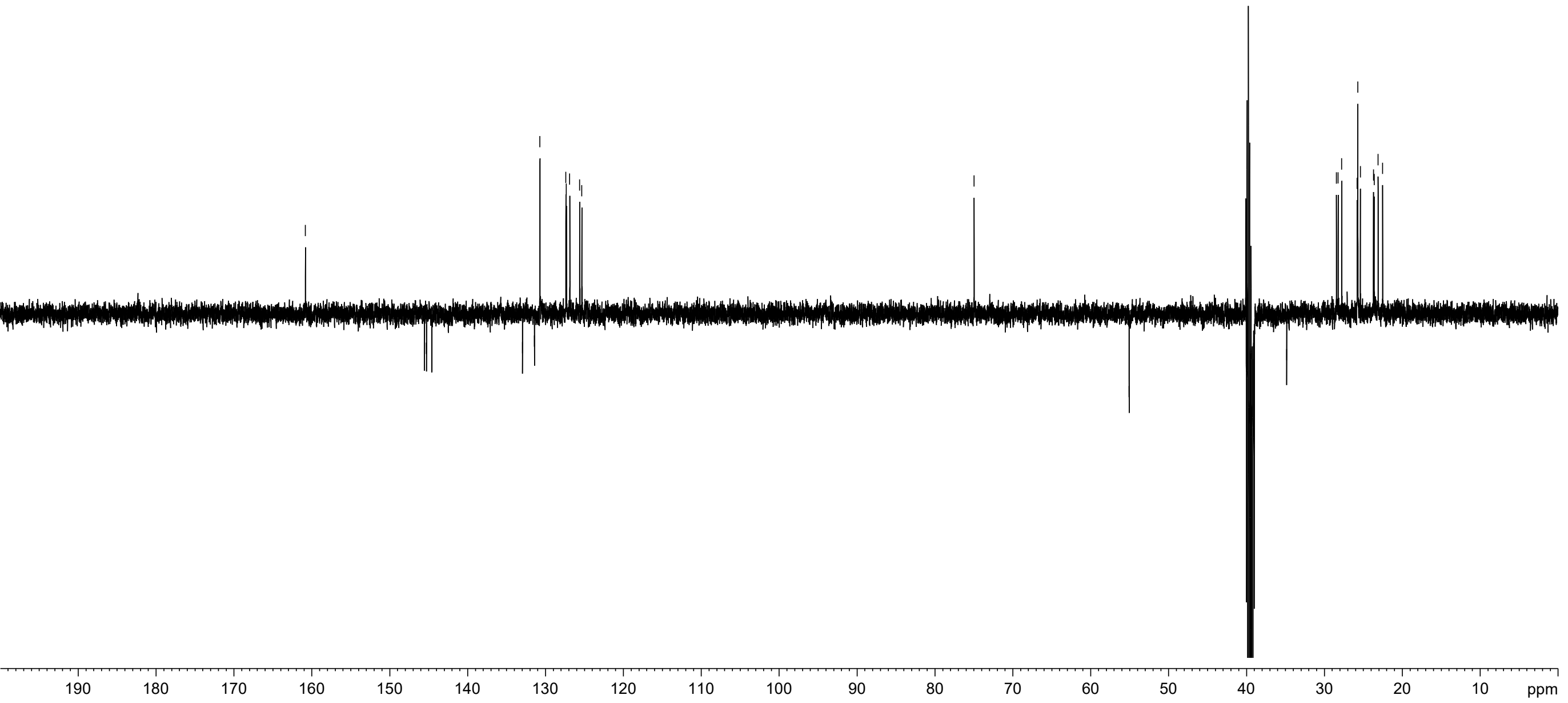



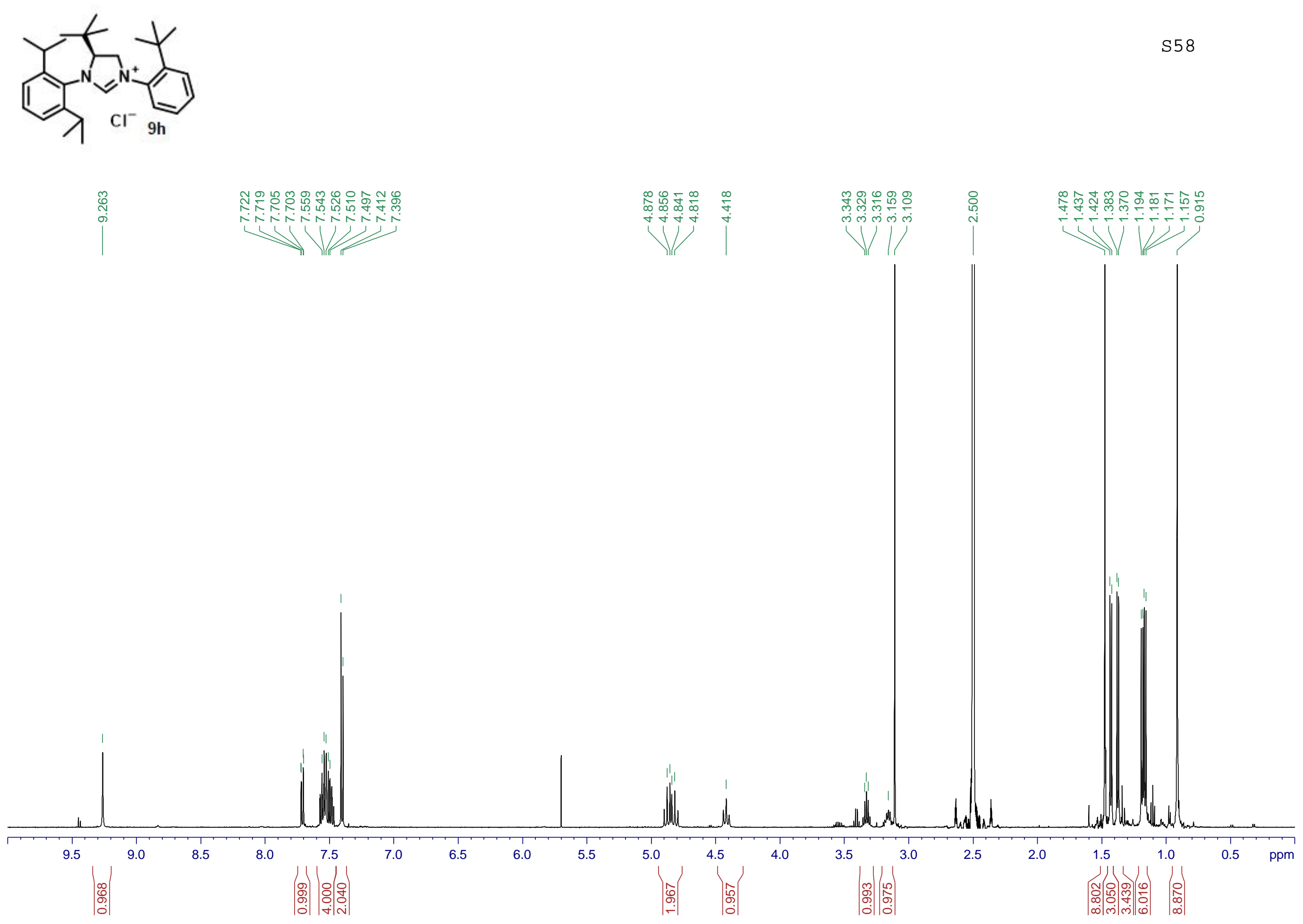

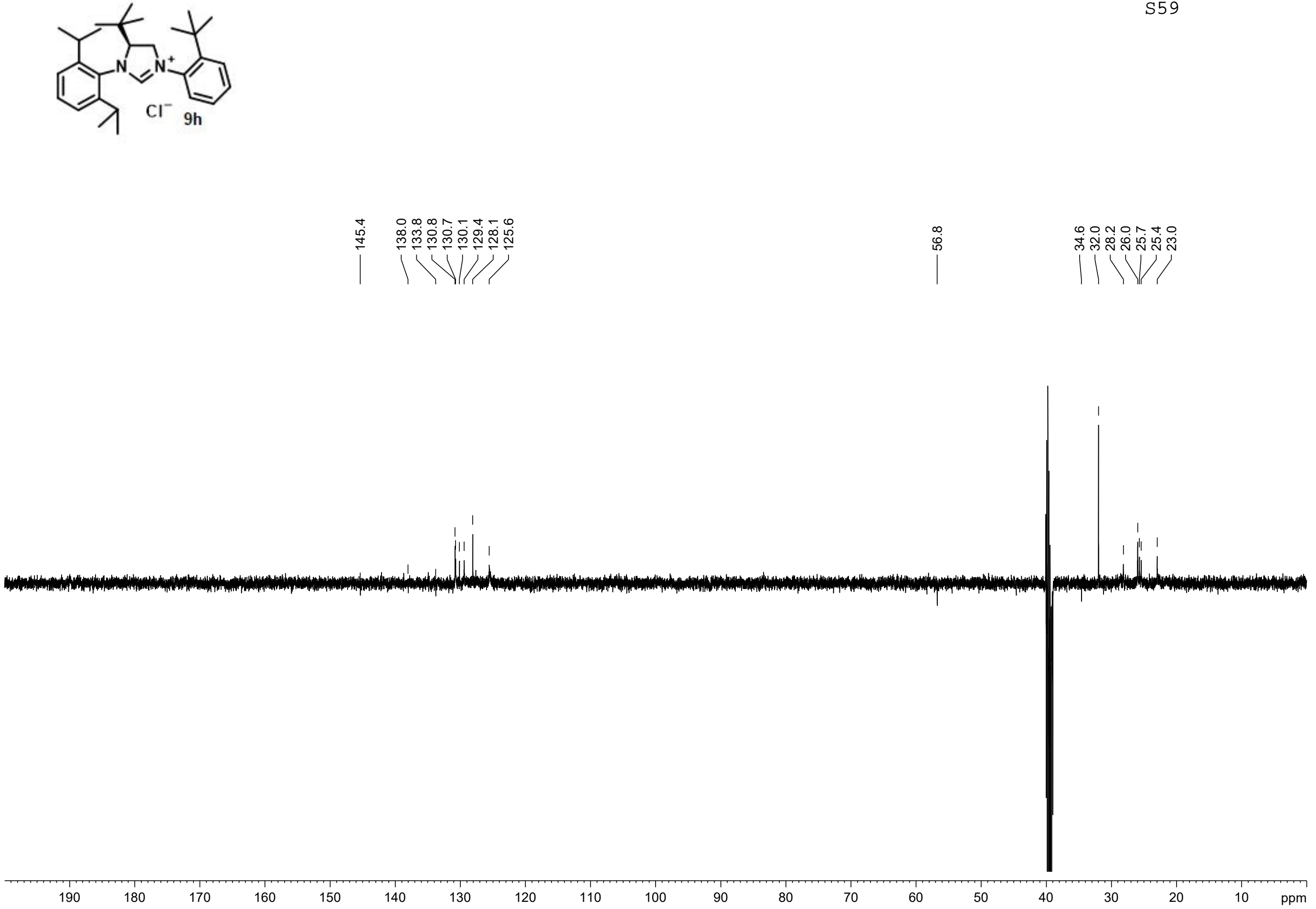

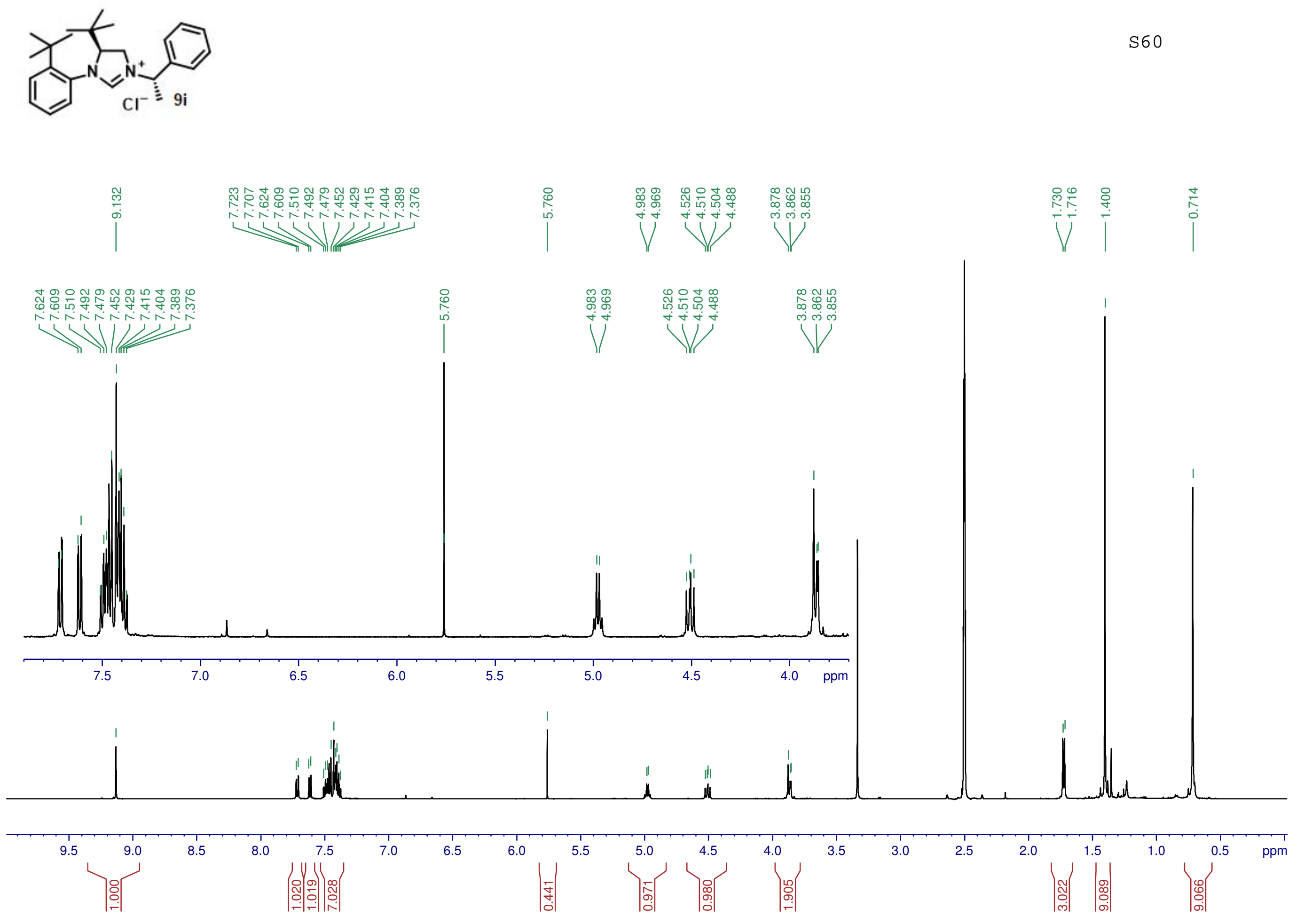


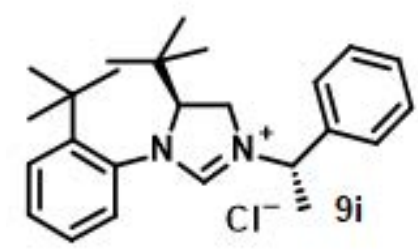

$\left.\right|^{\substack{0 \\ \underline{m}}}$

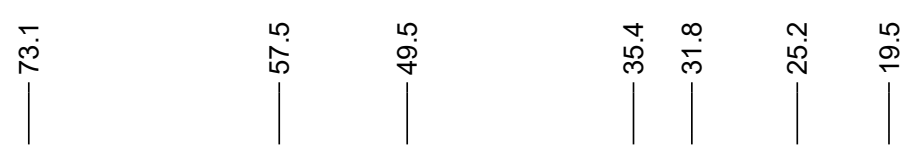

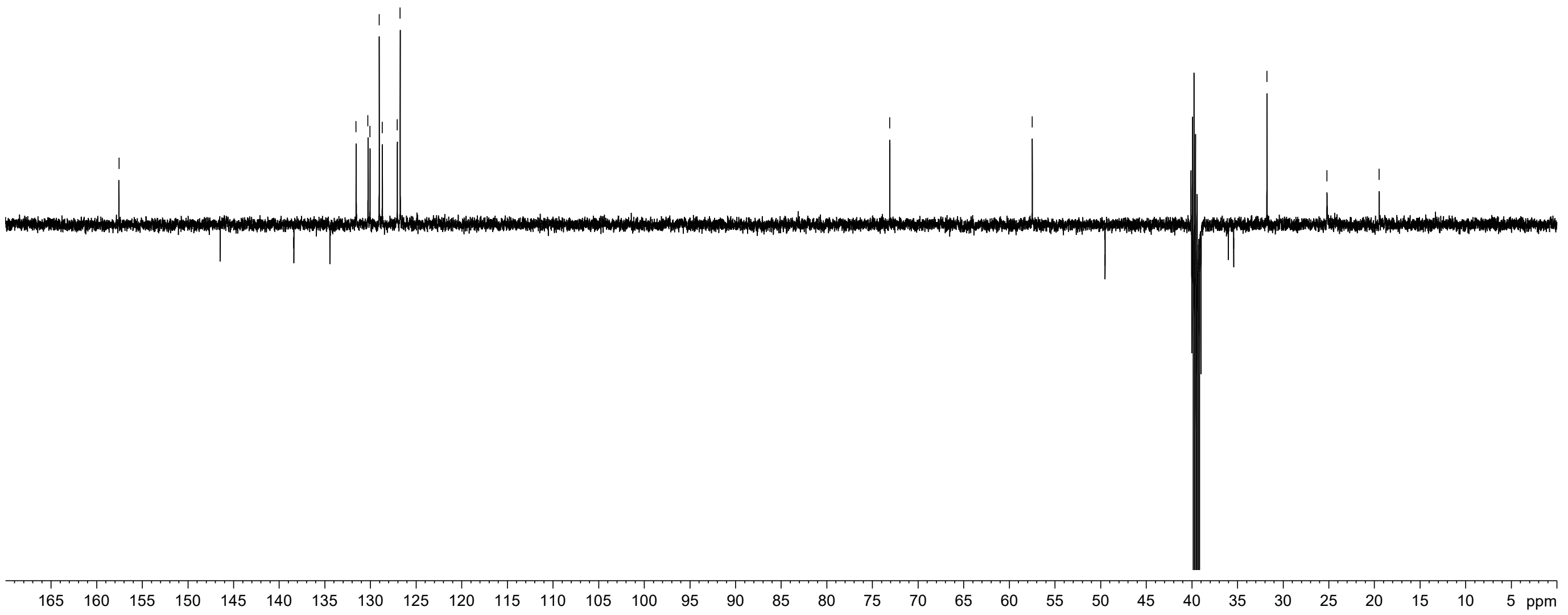



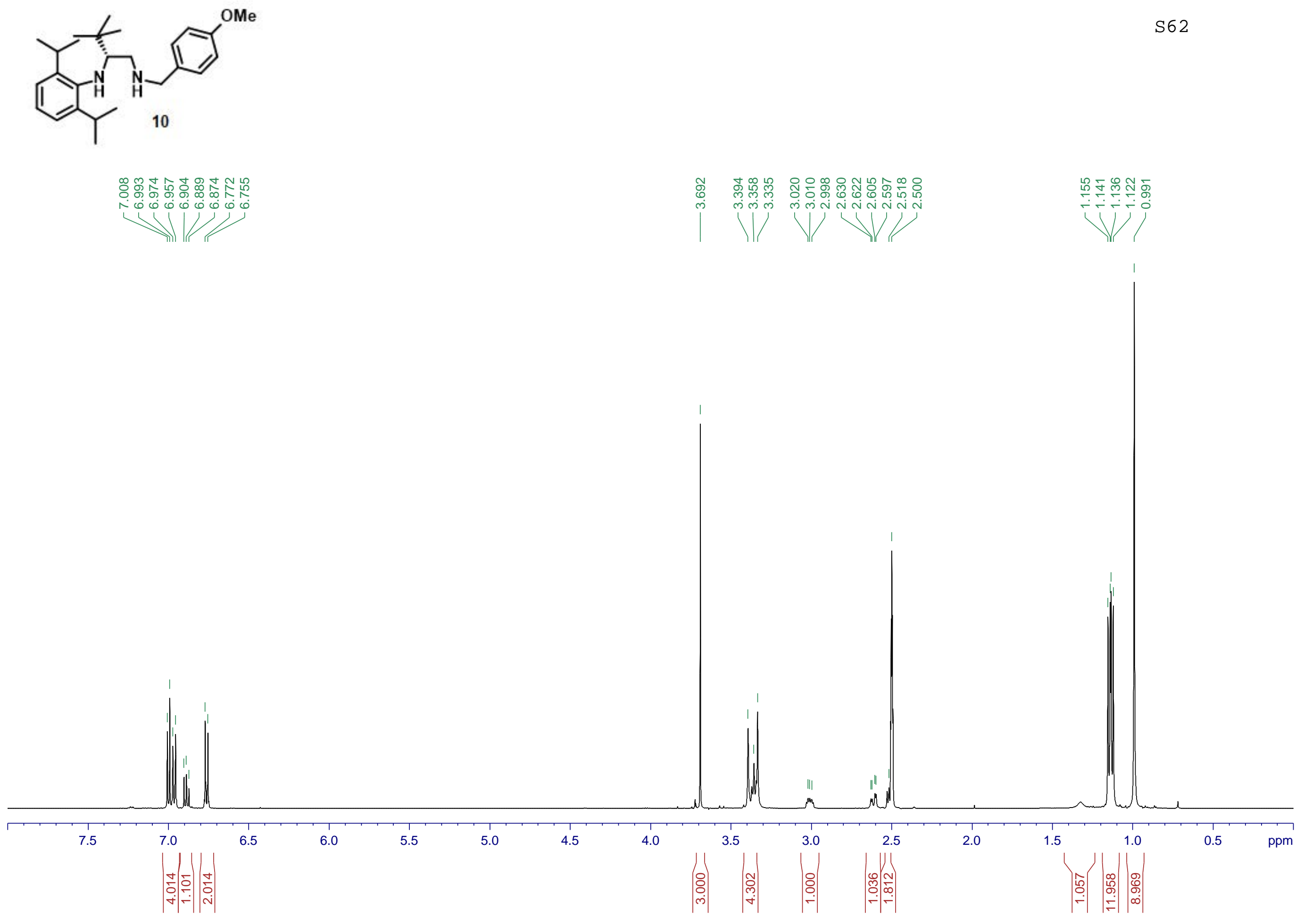

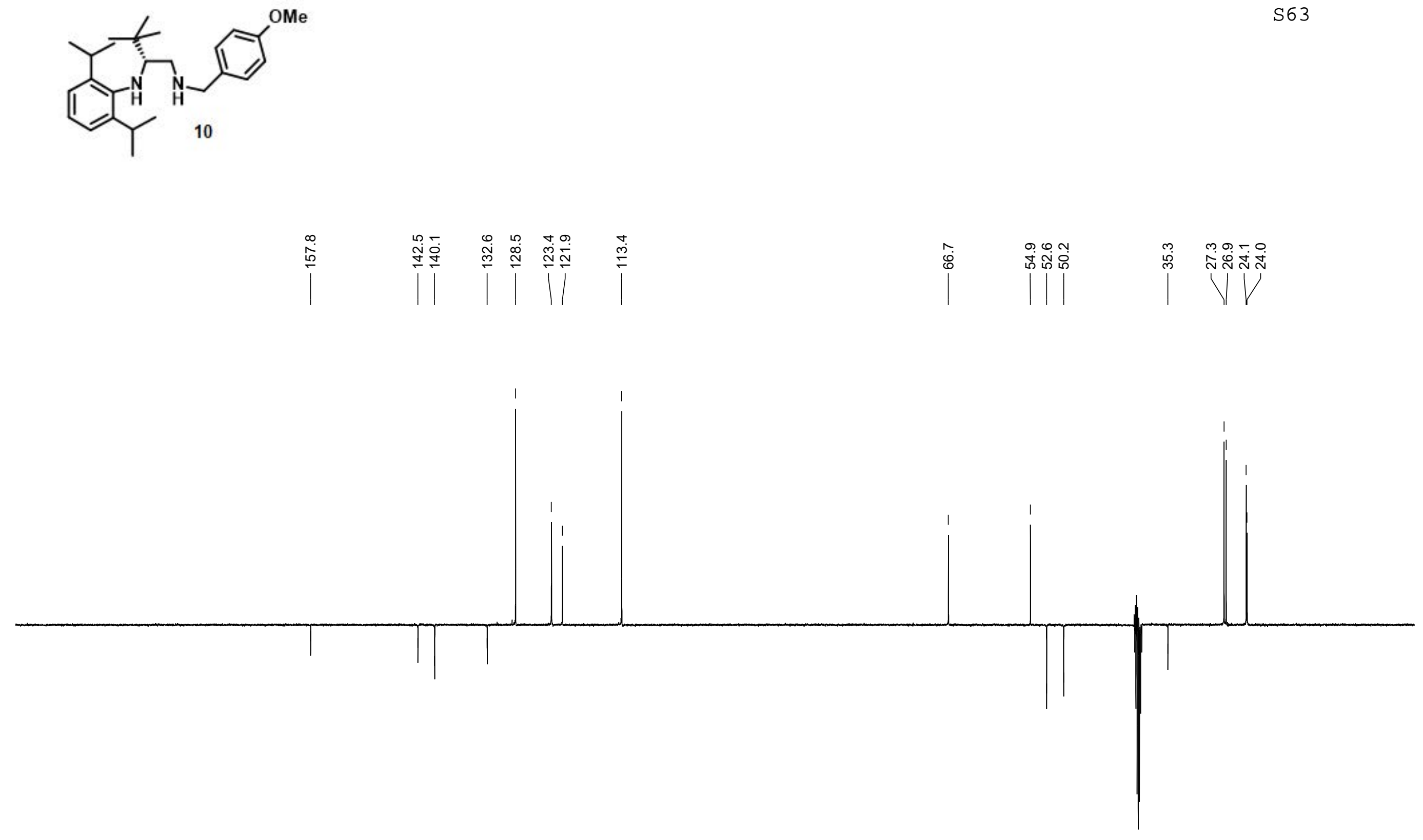

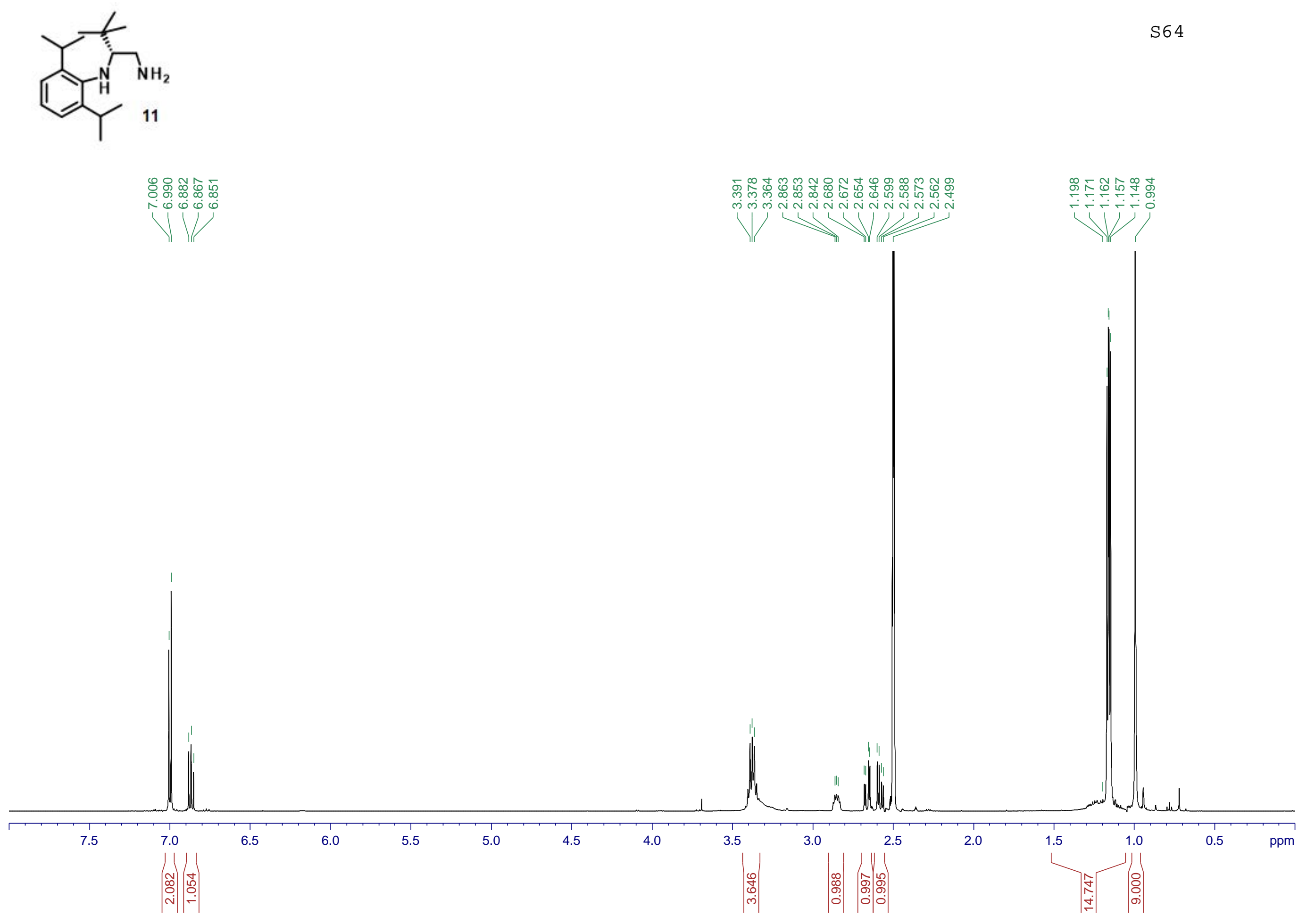


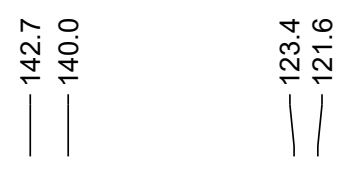

ஷ্ं



1

$\sqrt{n}$

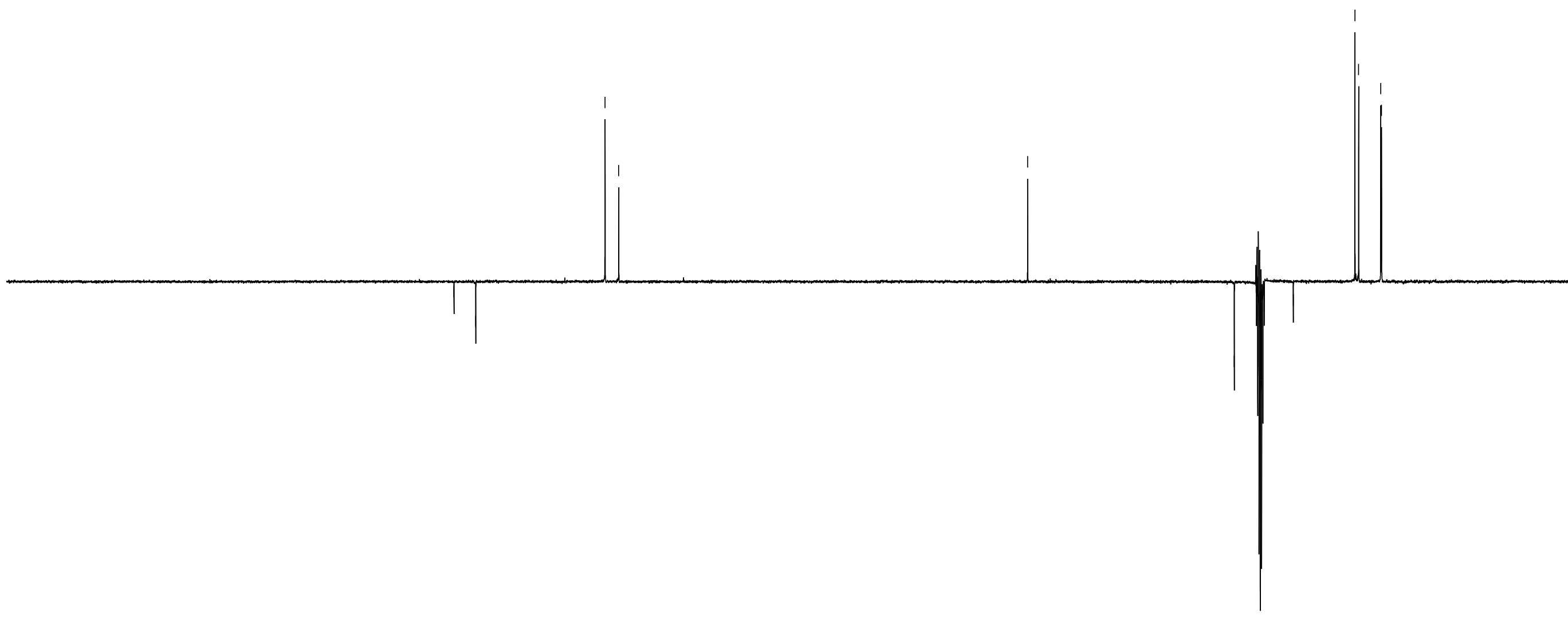



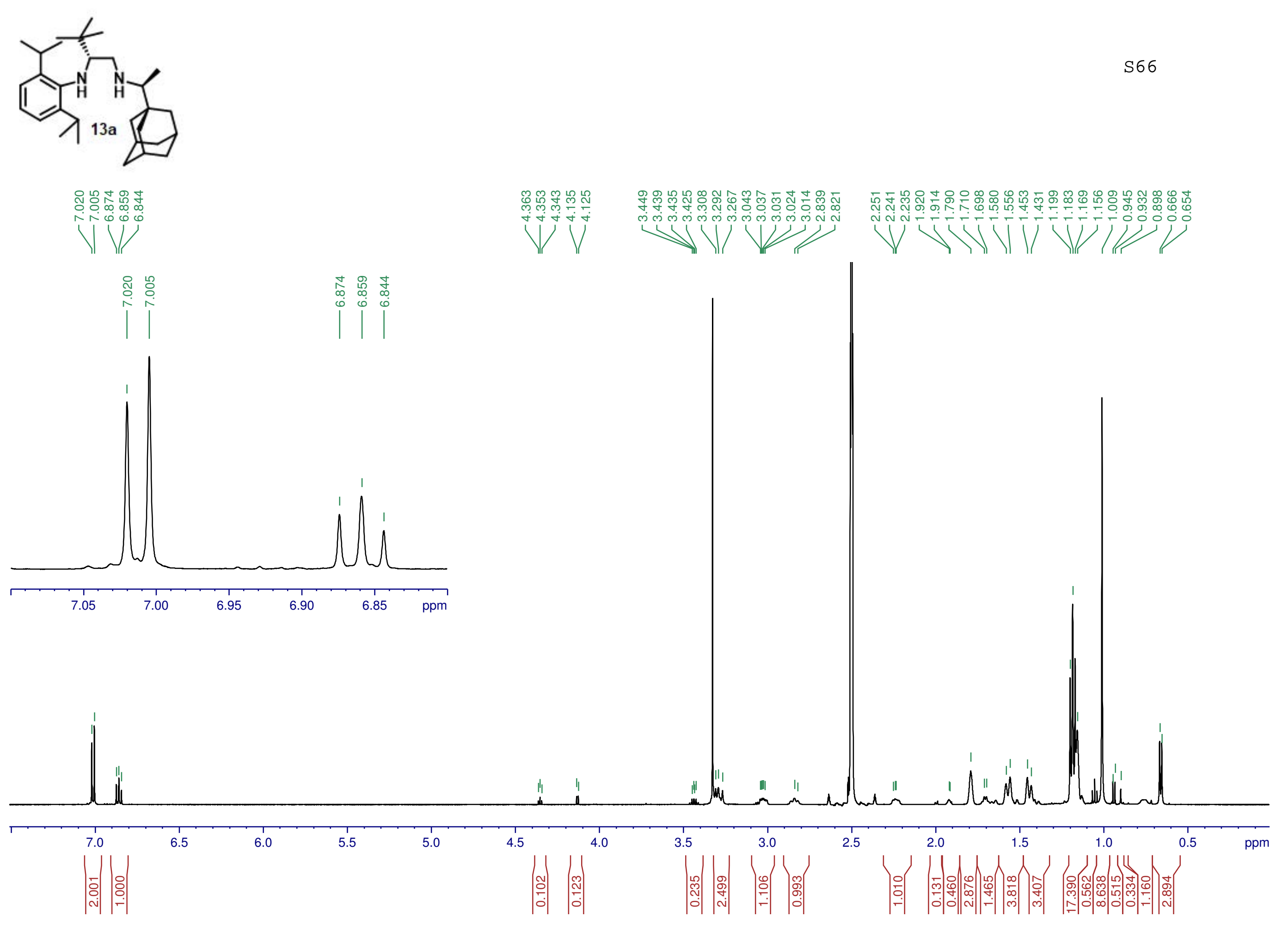

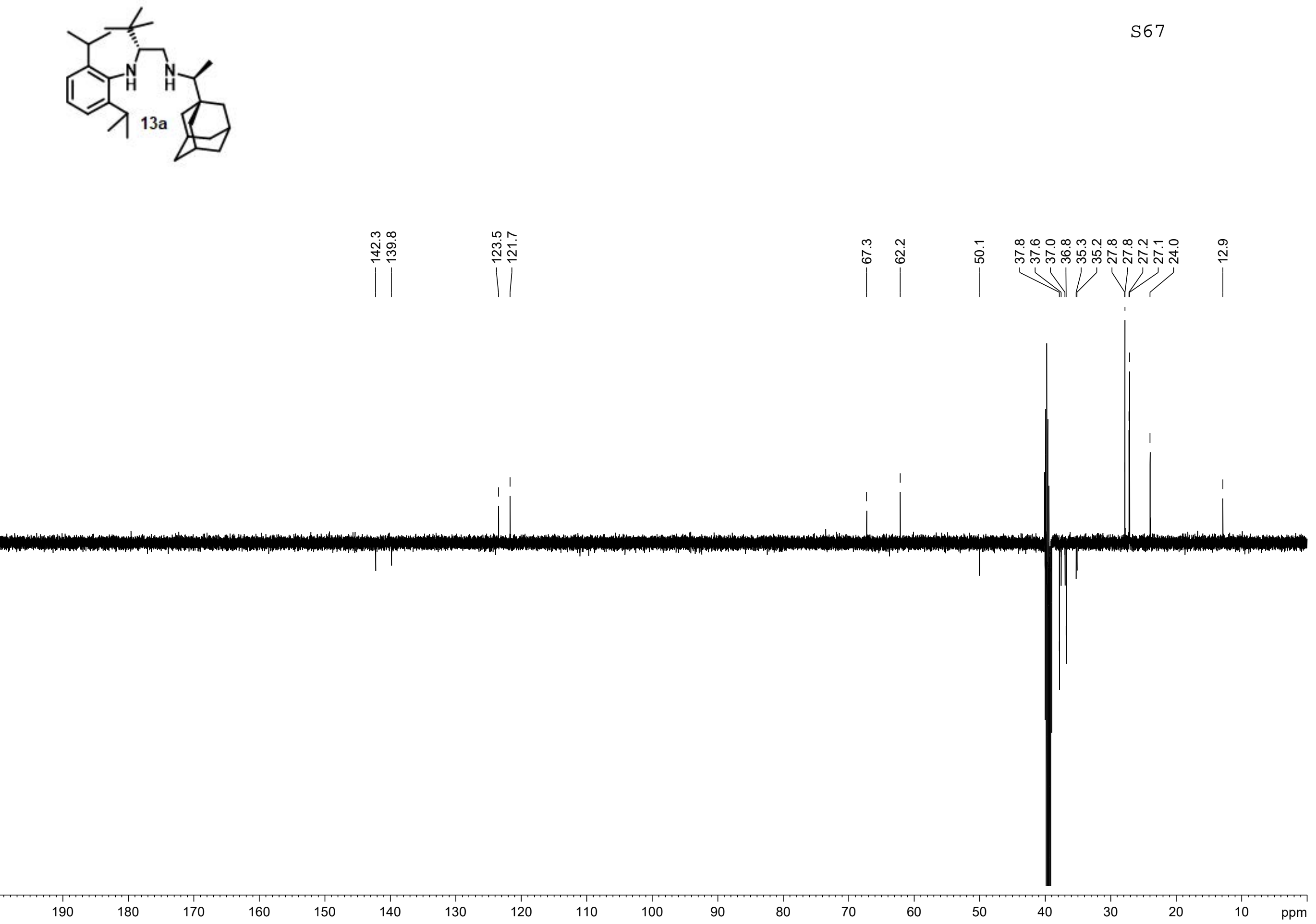


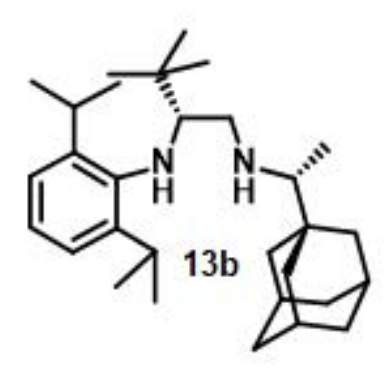

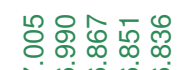

\i
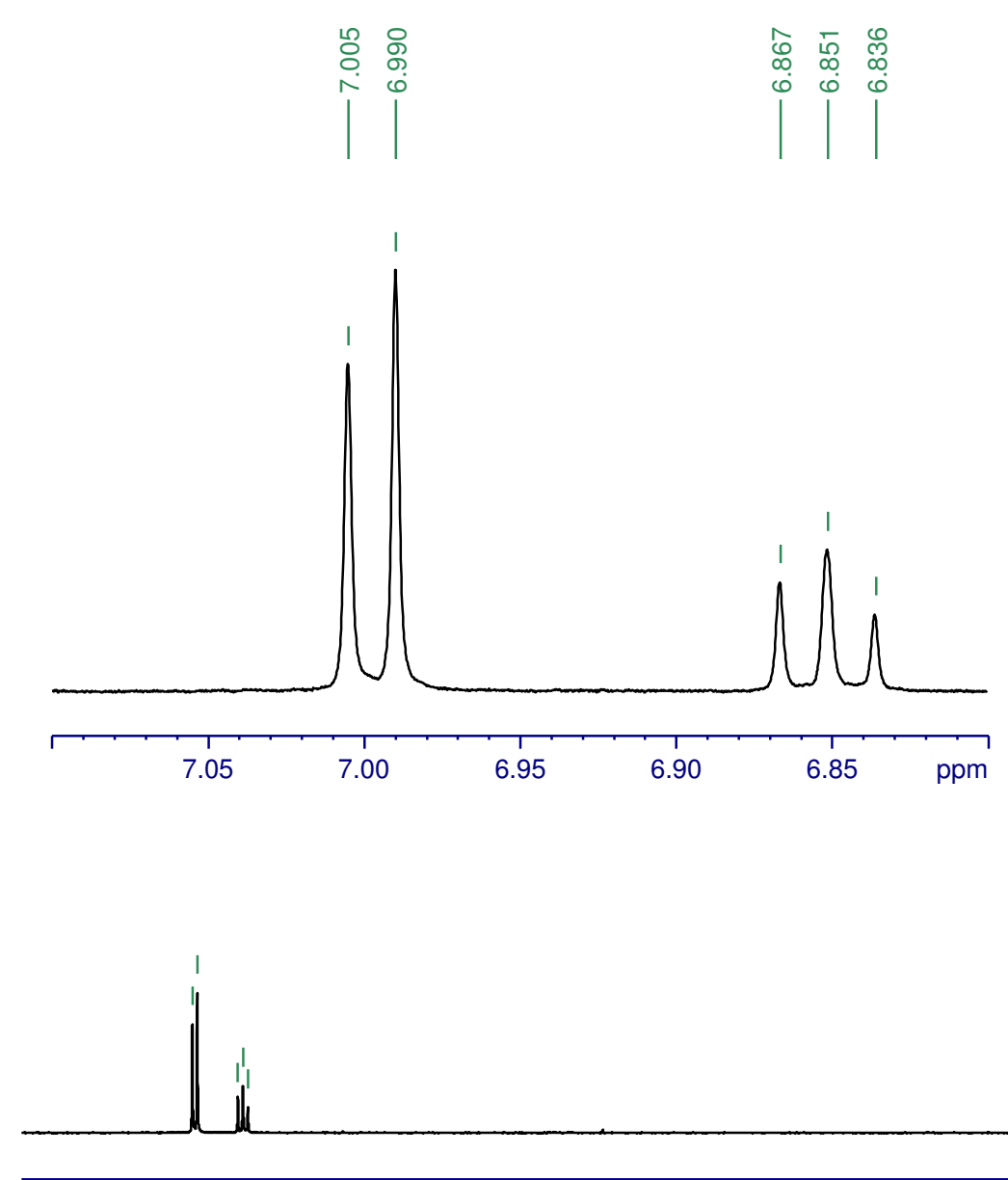
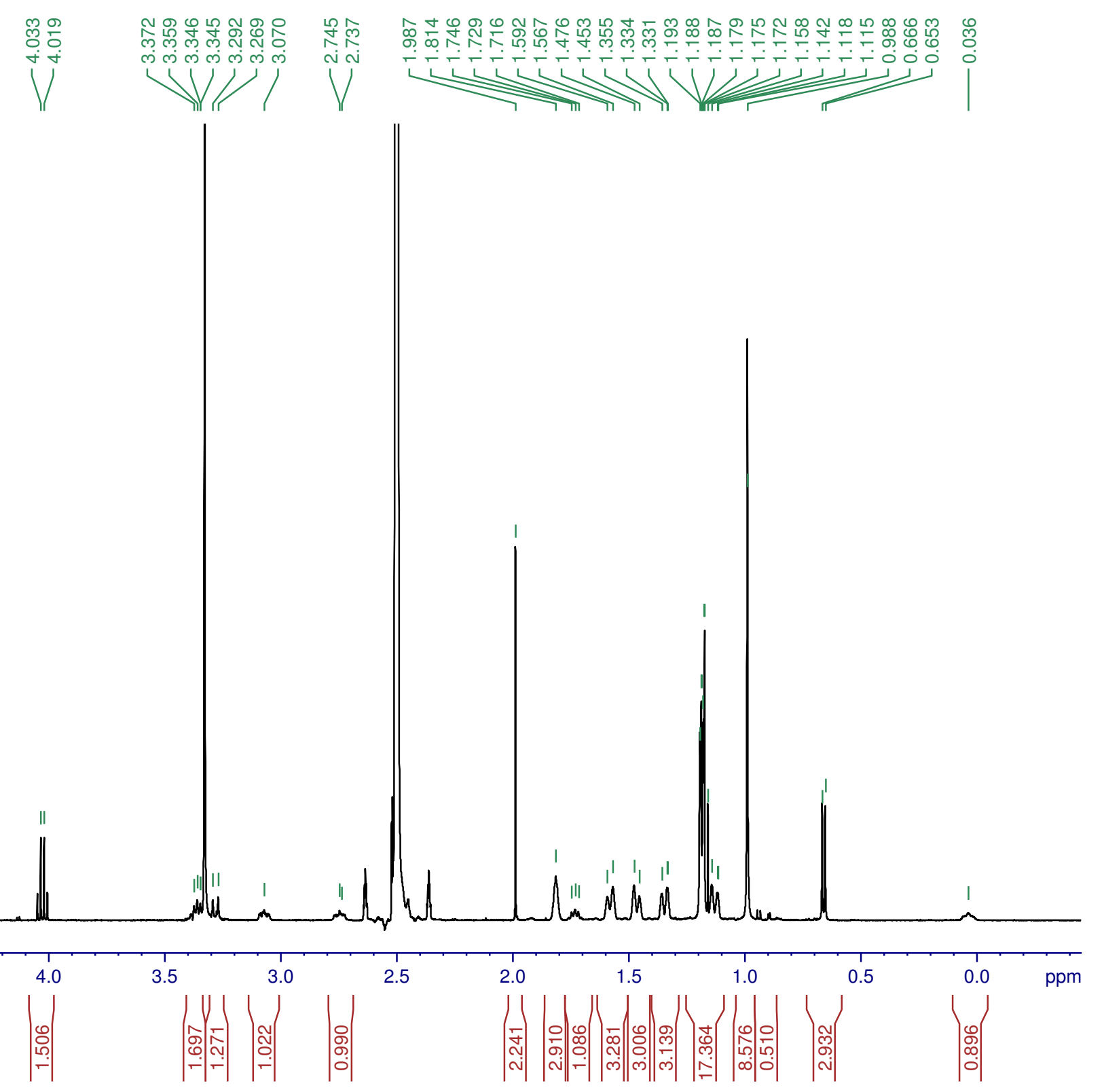


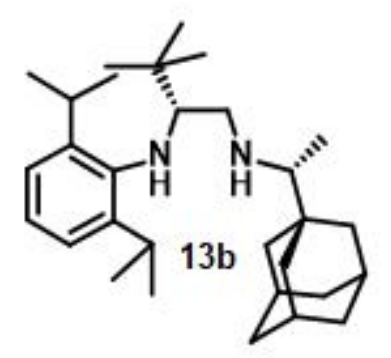

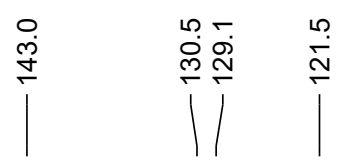

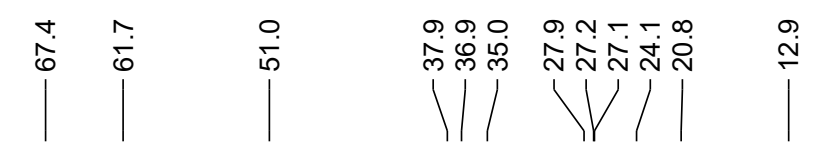

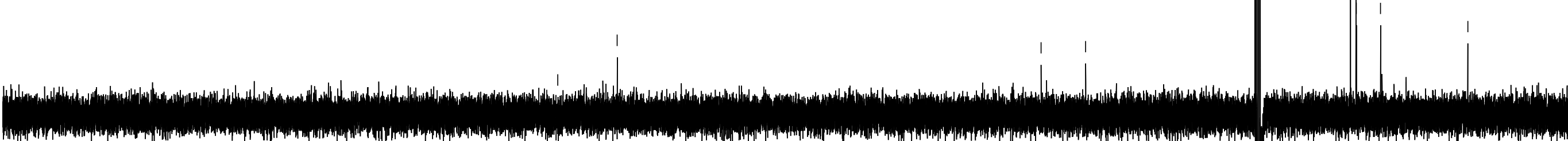

$140 \quad 130$




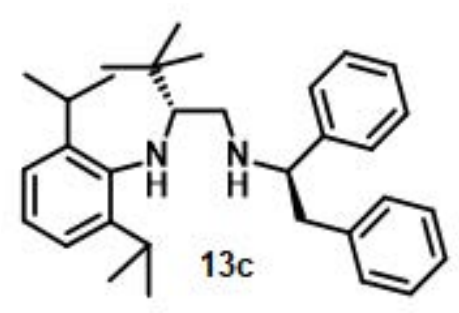

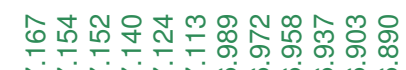

$\underbrace{r} \hat{n}$
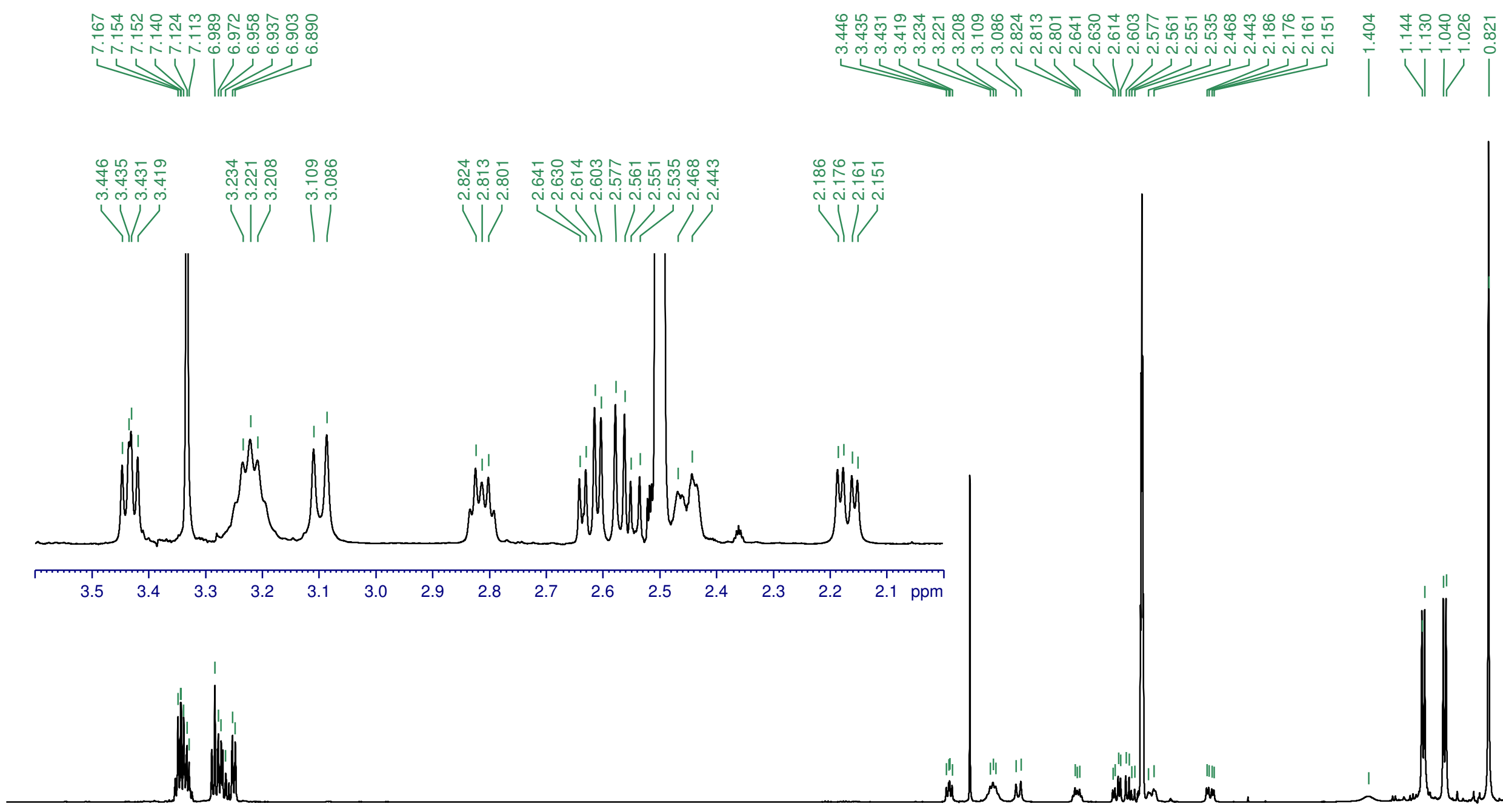

$M$

7.5

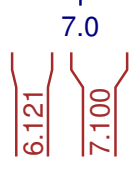

6.5

6.0

5.5

5.0

4.5

4.0

3.5

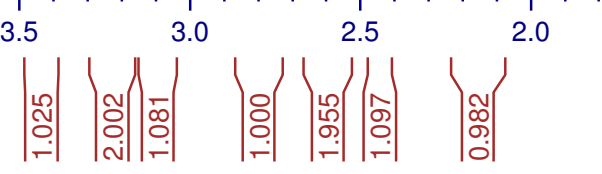

1.5

$1 / 1.0$

$0.5 \quad \mathrm{ppm}$

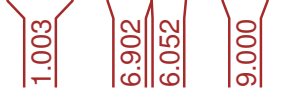




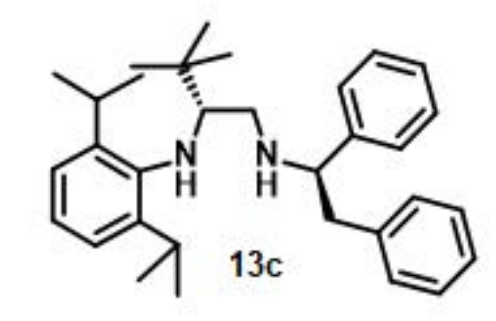

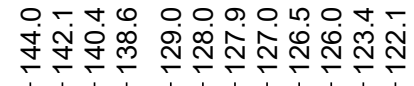
IIT WIII

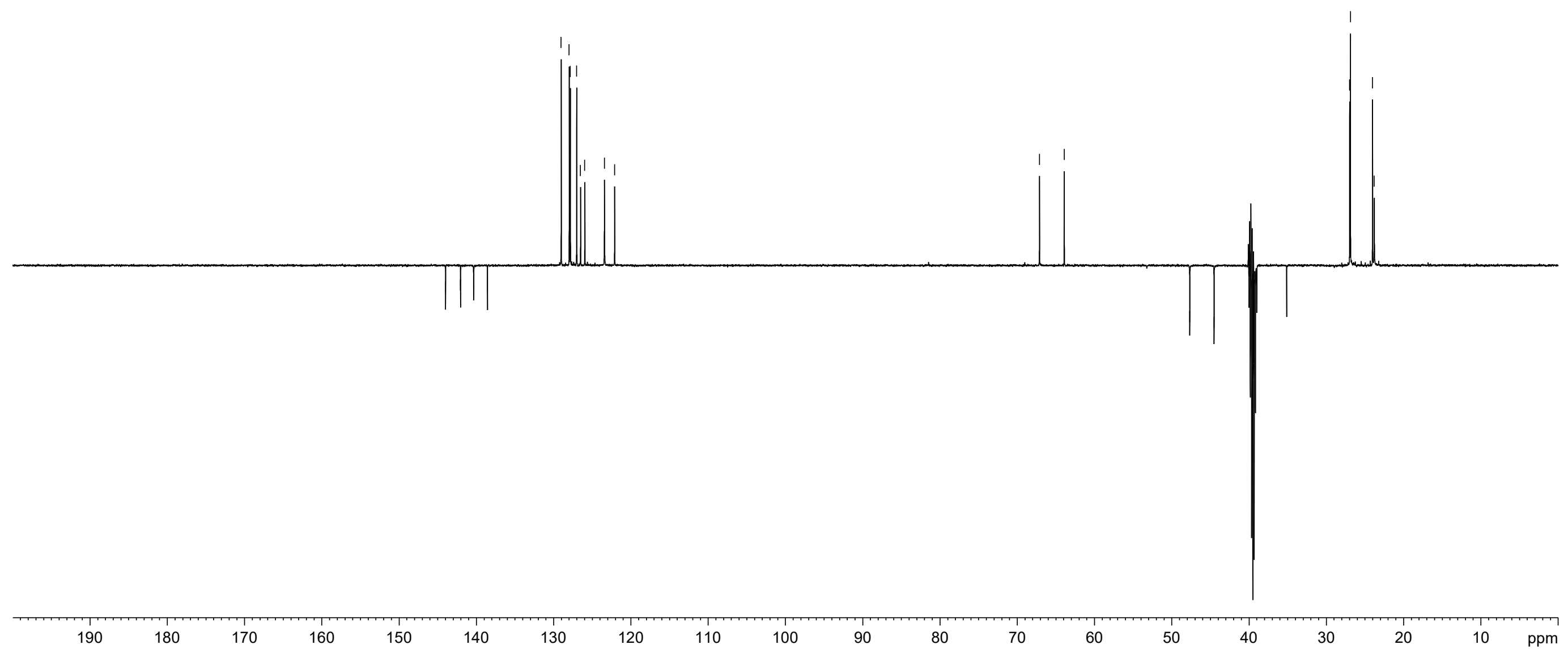



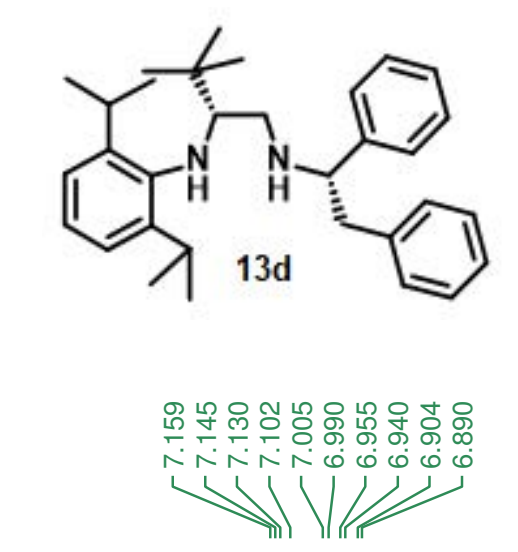

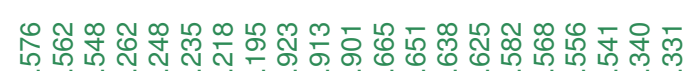
๓

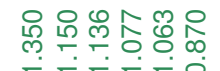
(1)
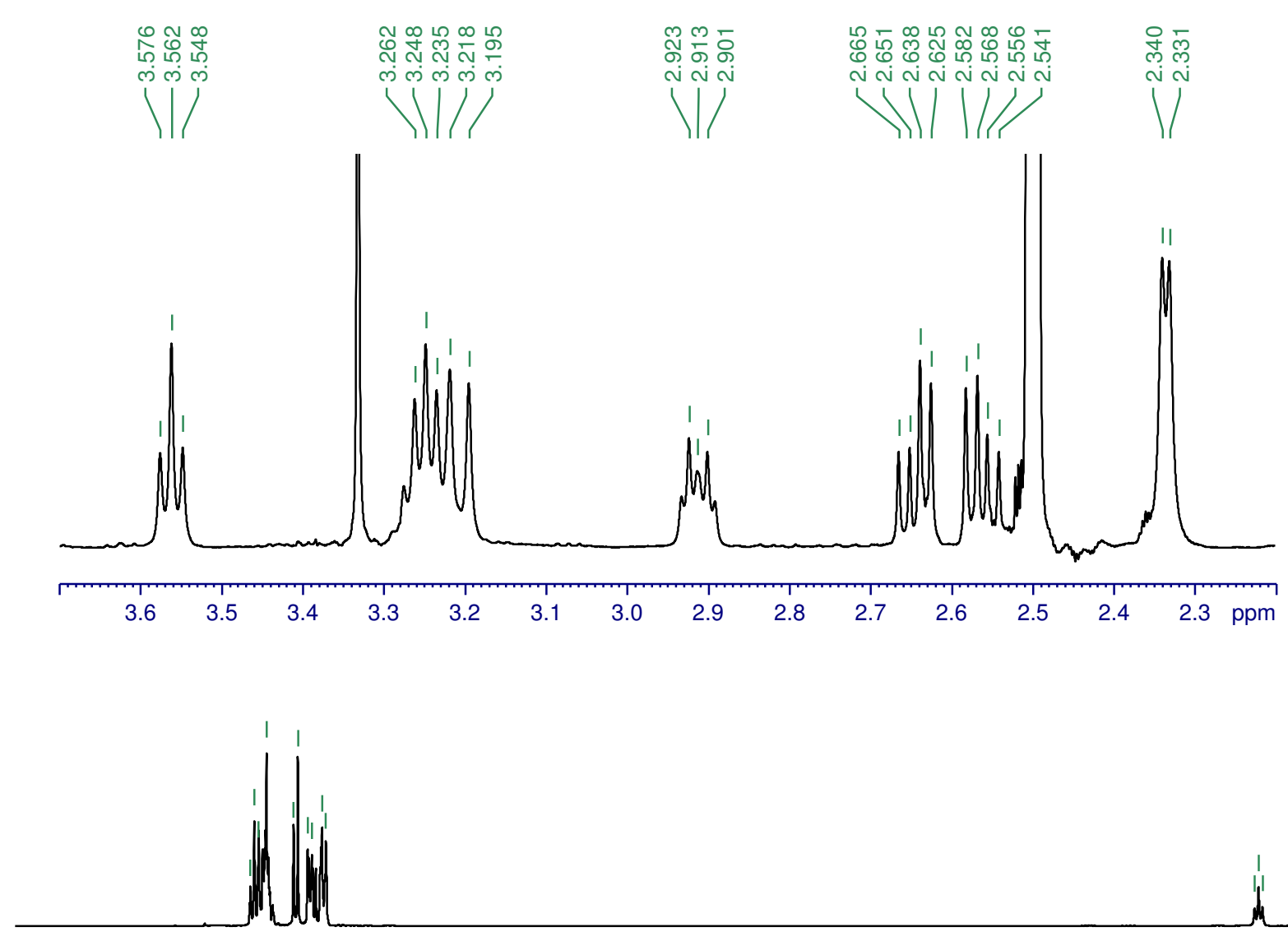

Lincidi

\begin{tabular}{|c|c|c|c|c|c|c|c|c|c|c|c|c|c|c|c|c|c|}
\hline 7.5 & 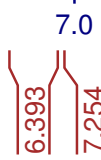 & 6.5 & 6.0 & 5.5 & 5.0 & 4.5 & 4.0 &  & $\left|\begin{array}{l}\text { o } \\
\text { ํㅣ }\end{array}\right|$ & $\begin{array}{l}3.0 \\
\left|\begin{array}{l}\infty \\
0 \\
0 \\
\hdashline\end{array}\right|\end{array}$ & 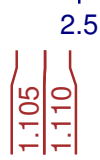 & $\left|\begin{array}{l}0 \\
0 \\
0 \\
\text { iv }\end{array}\right|$ & 2.0 & $\begin{array}{c}1.5 \\
\end{array}$ & 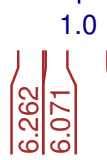 & 0.5 & ppm \\
\hline
\end{tabular}



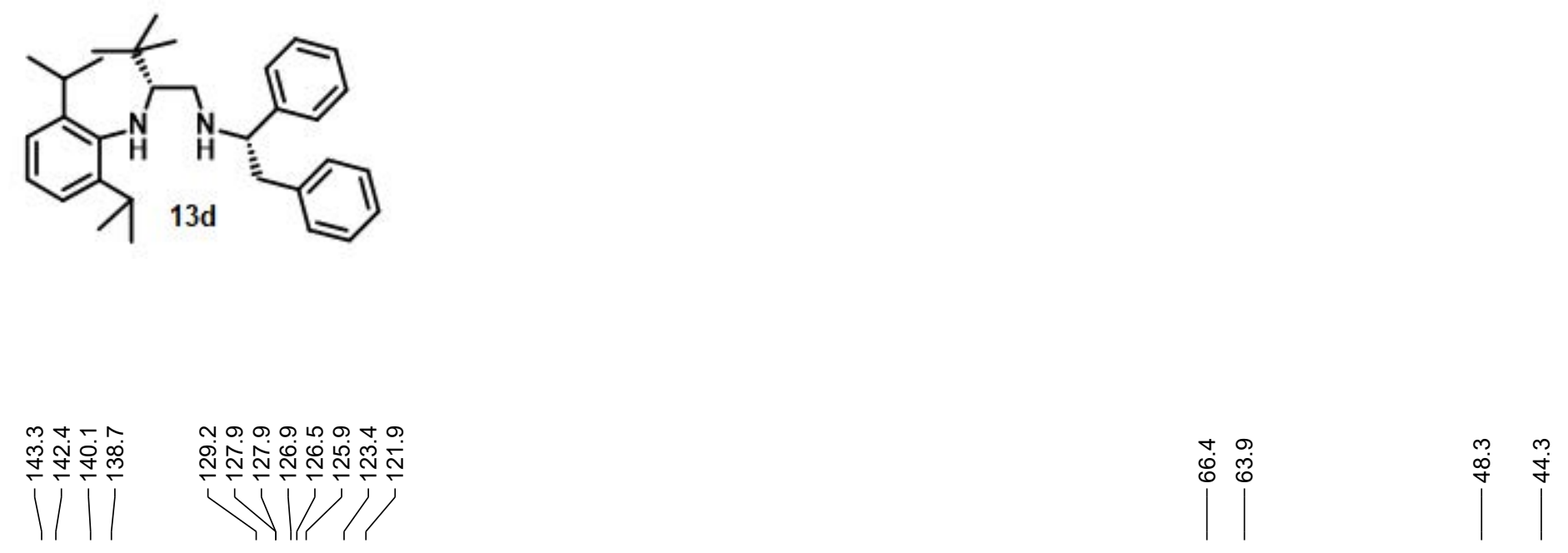

S73

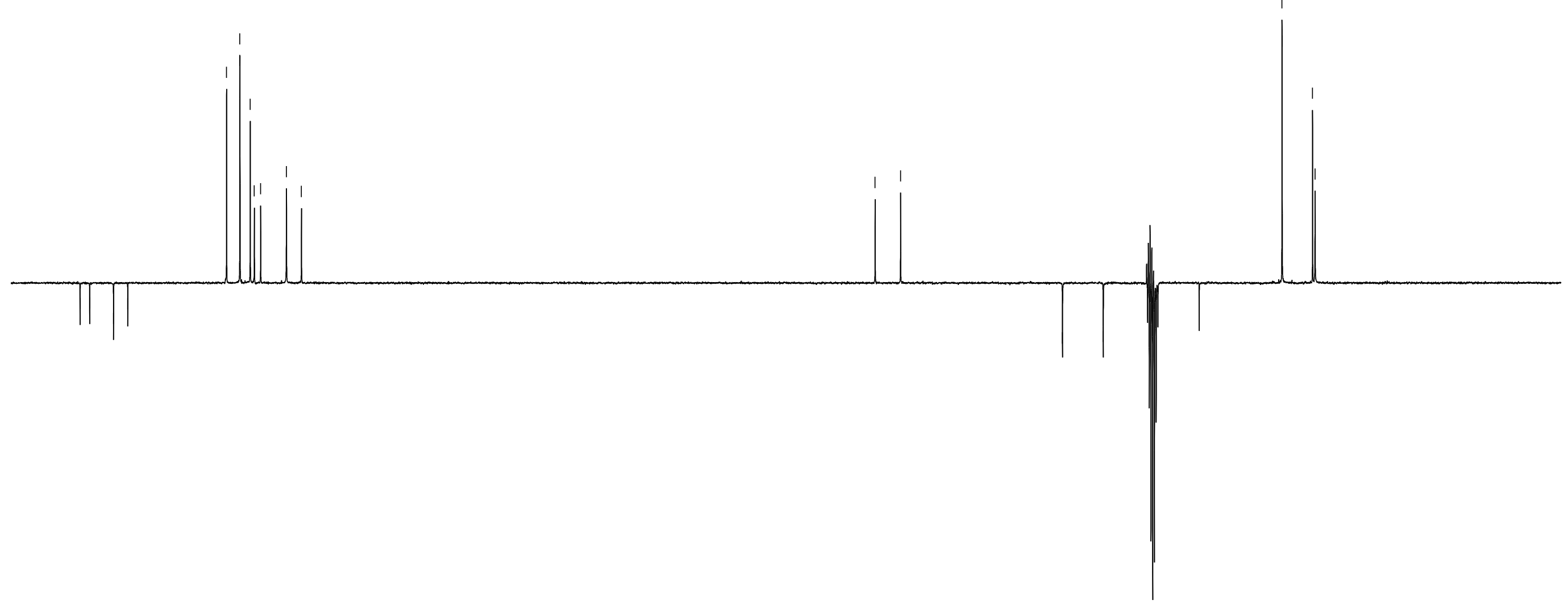

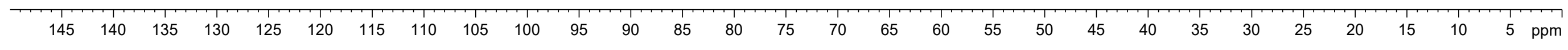




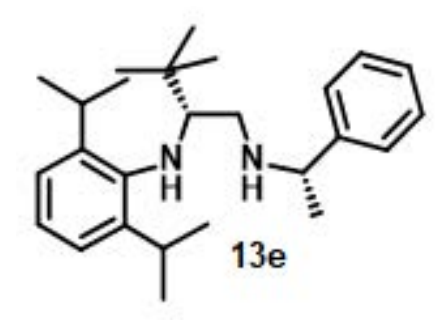

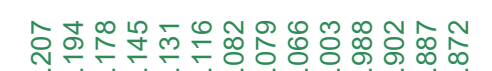

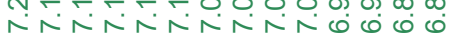

$\longrightarrow$
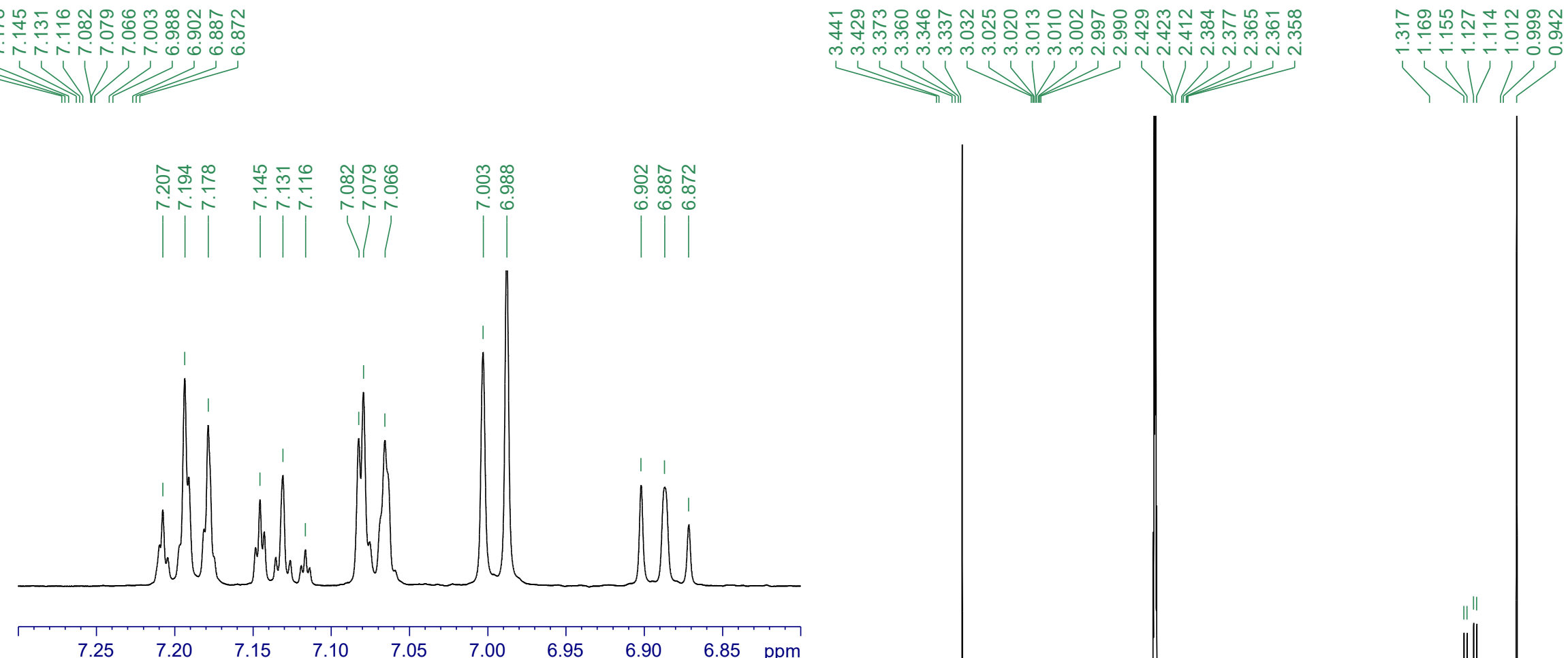

7.05

$7.00 \quad 6.95$

6.90
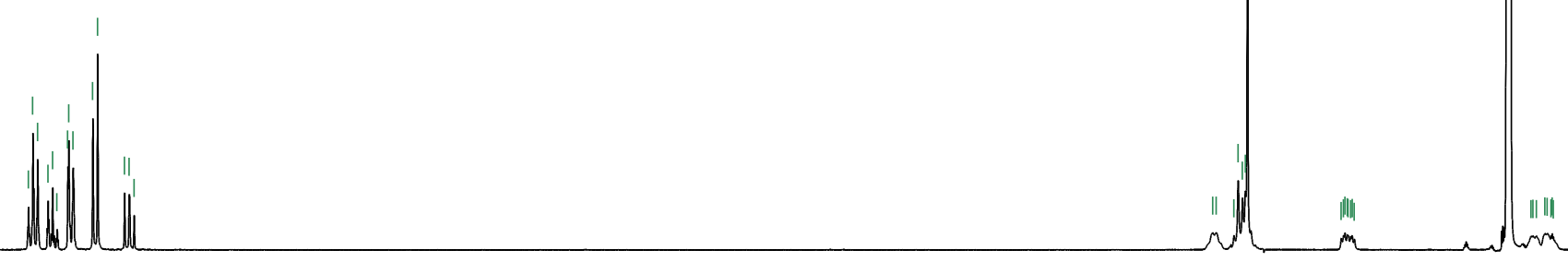

1 .
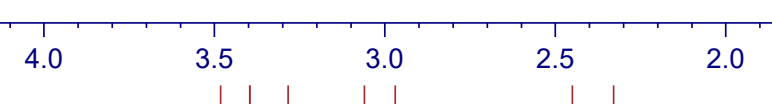


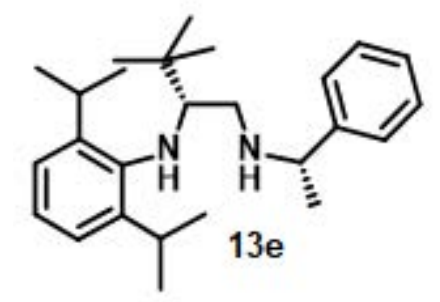

|
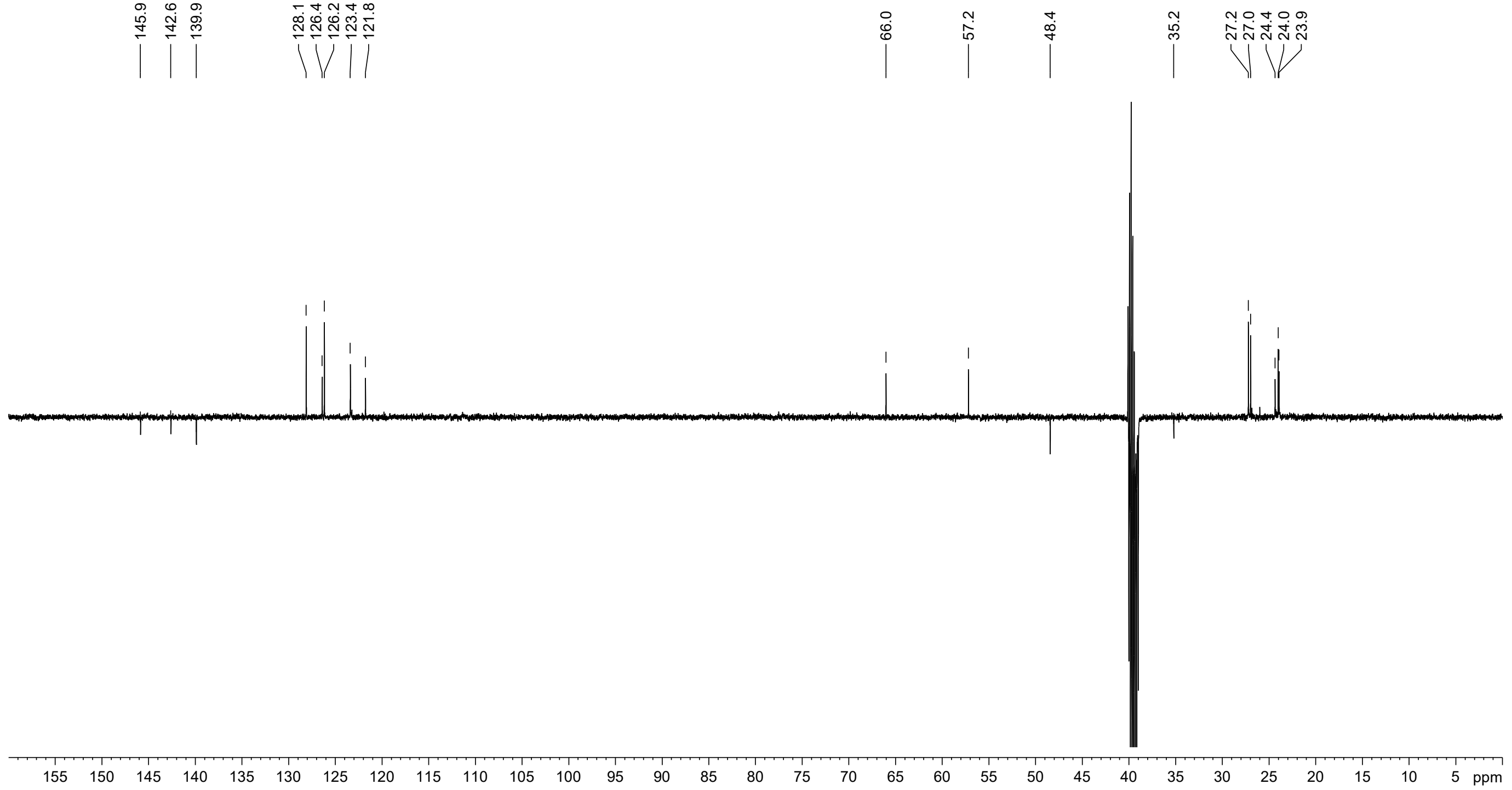


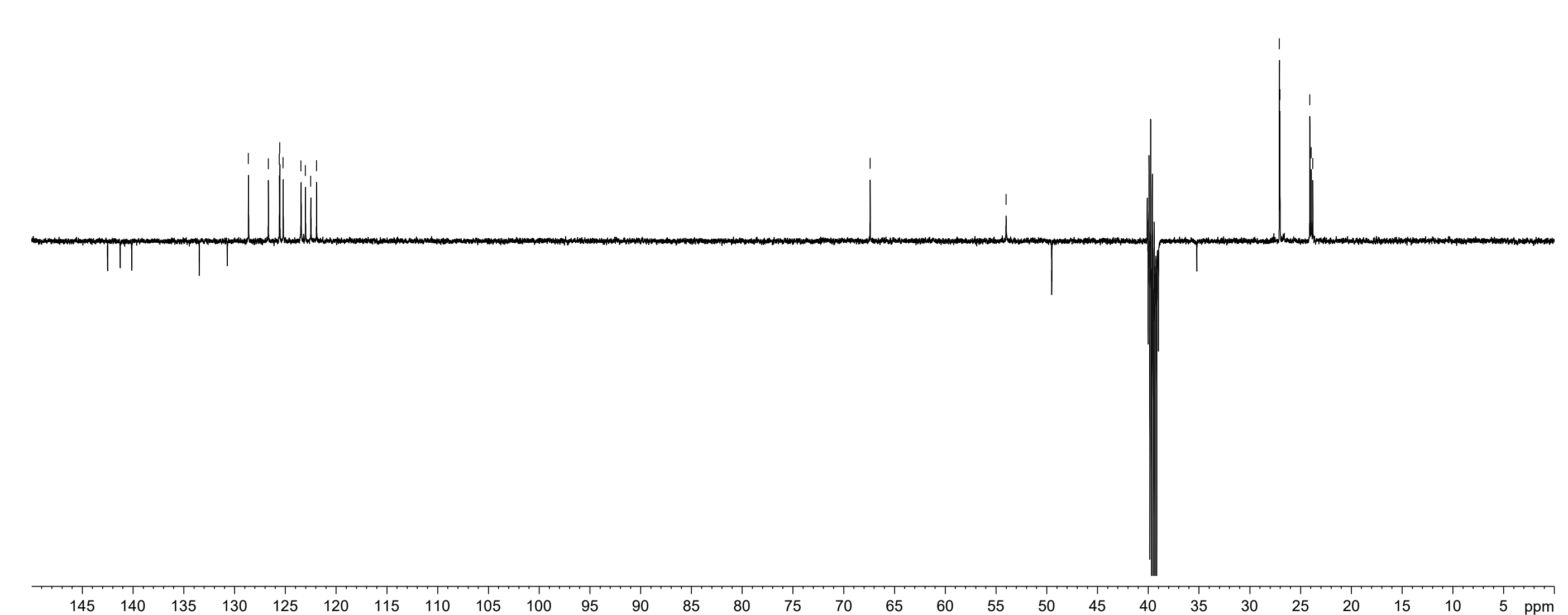




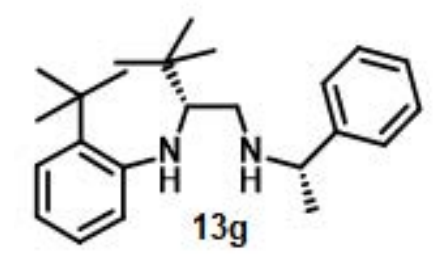

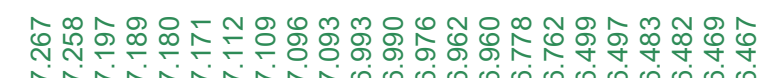

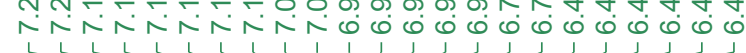
$\ldots$

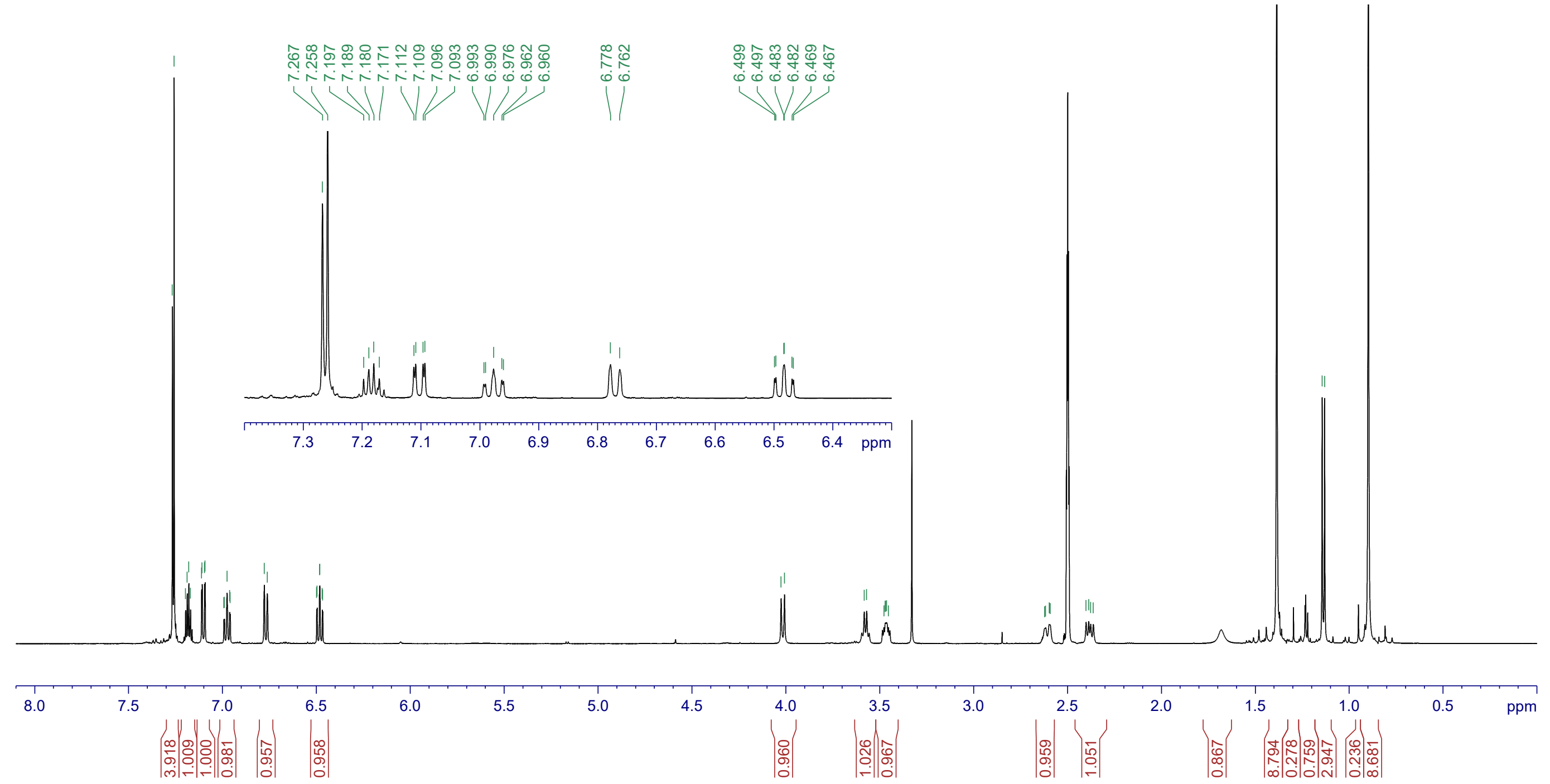



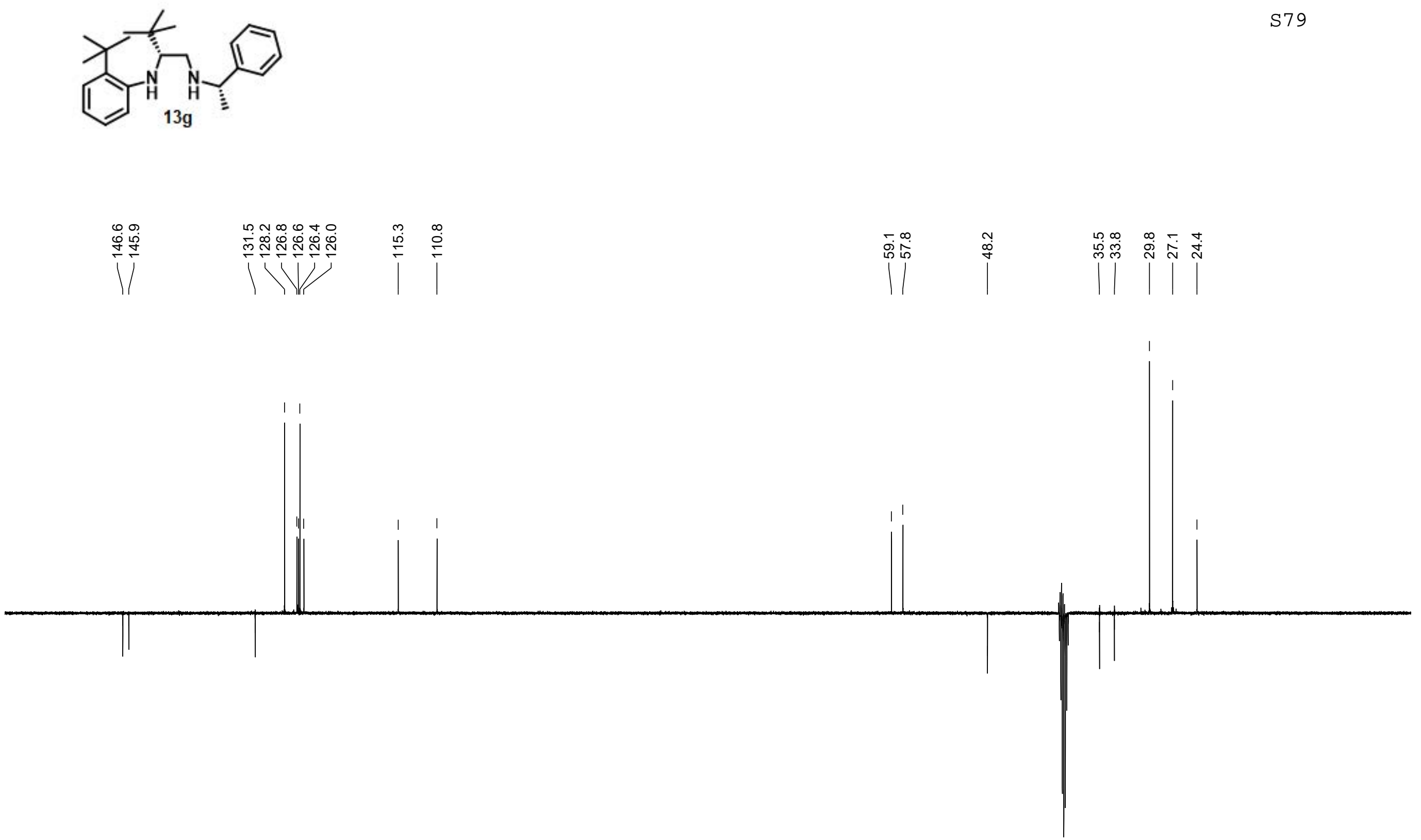

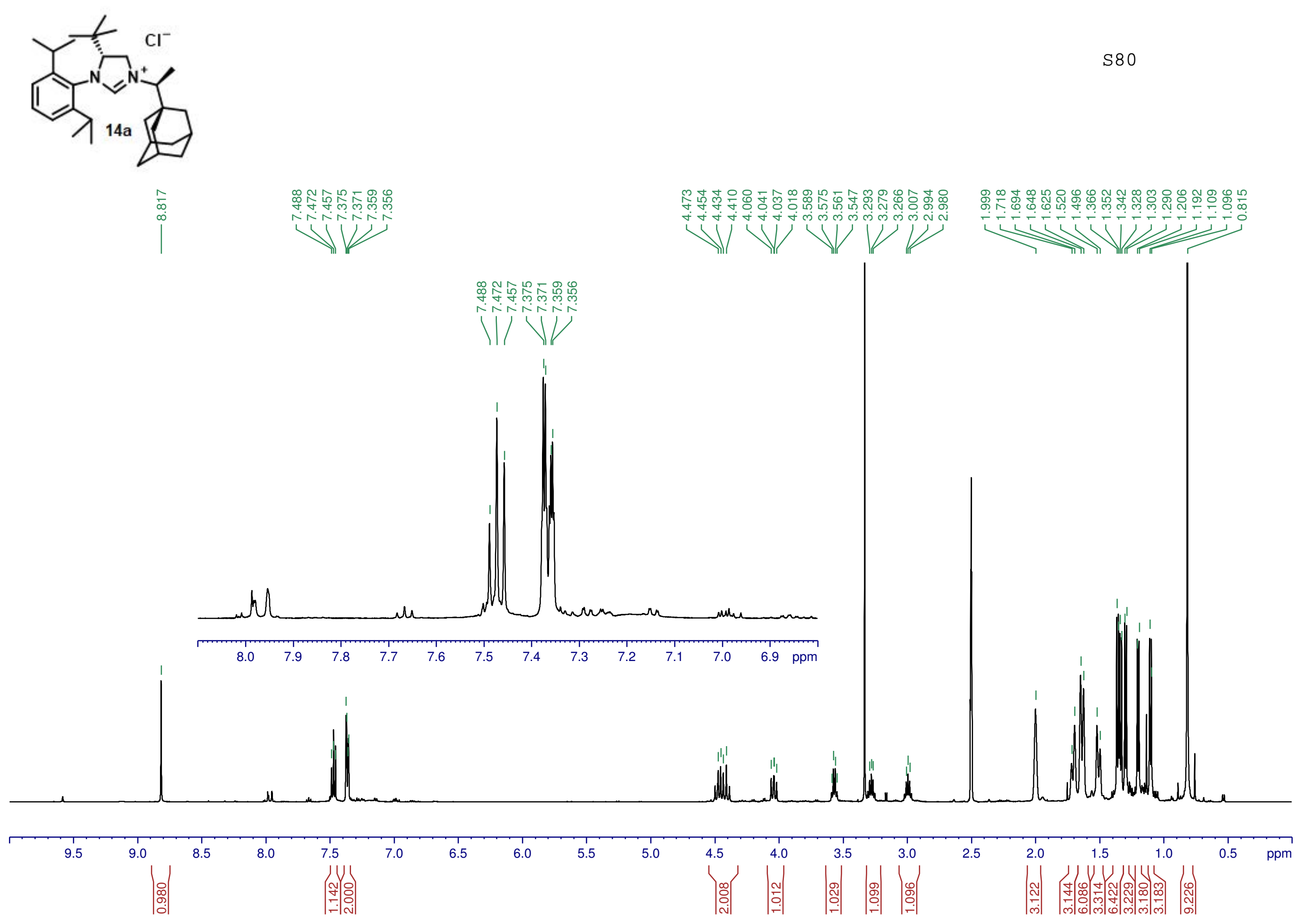

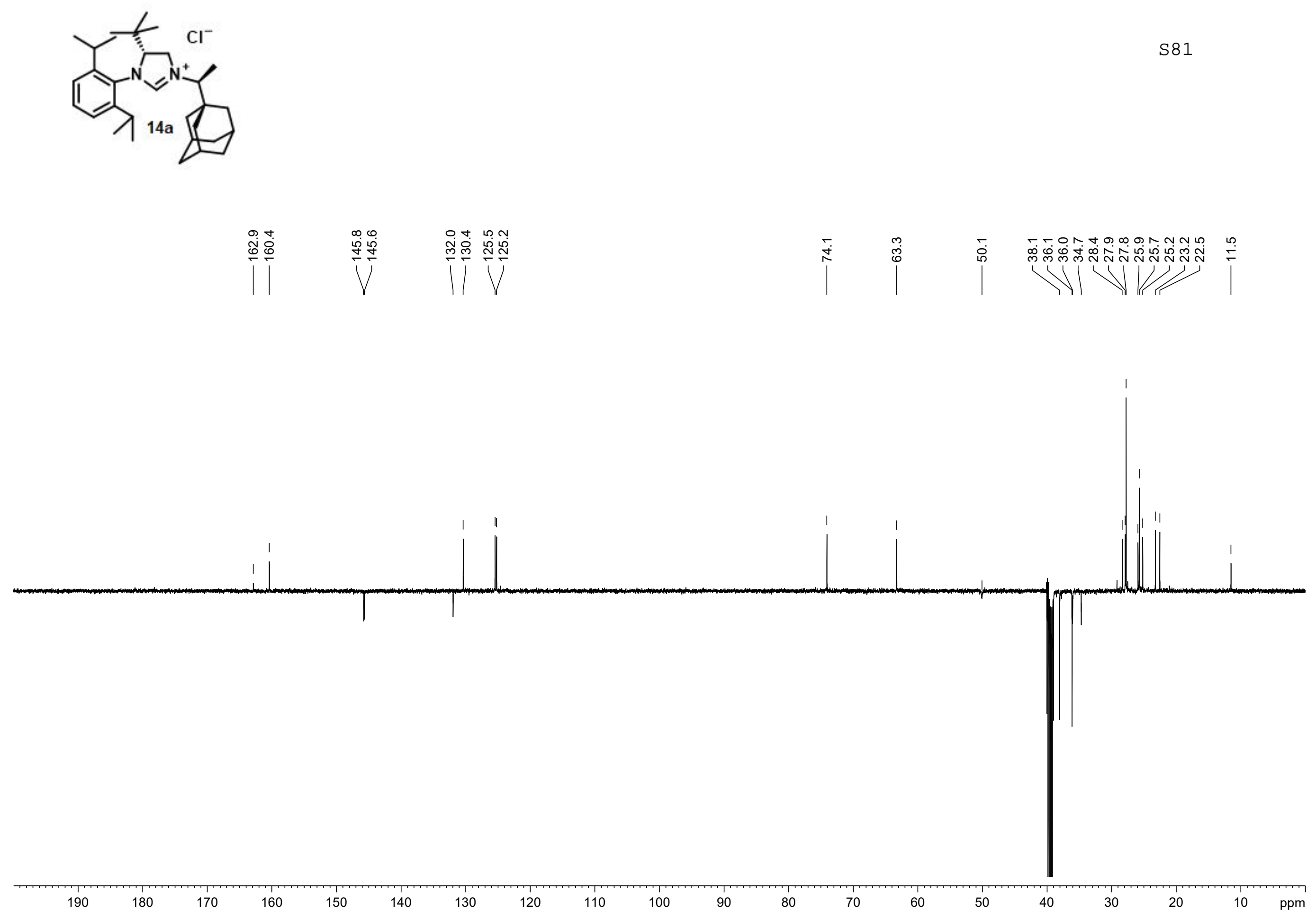

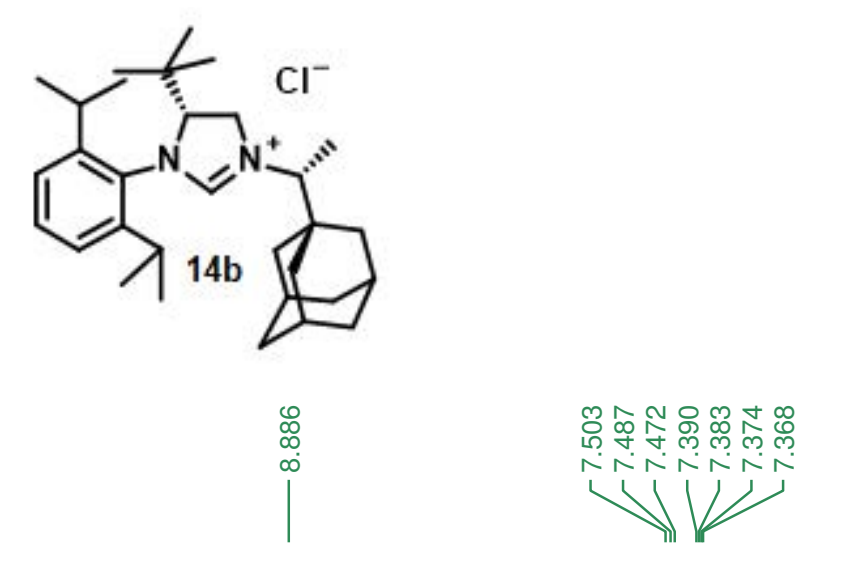

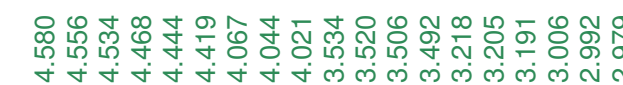

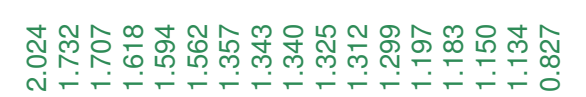
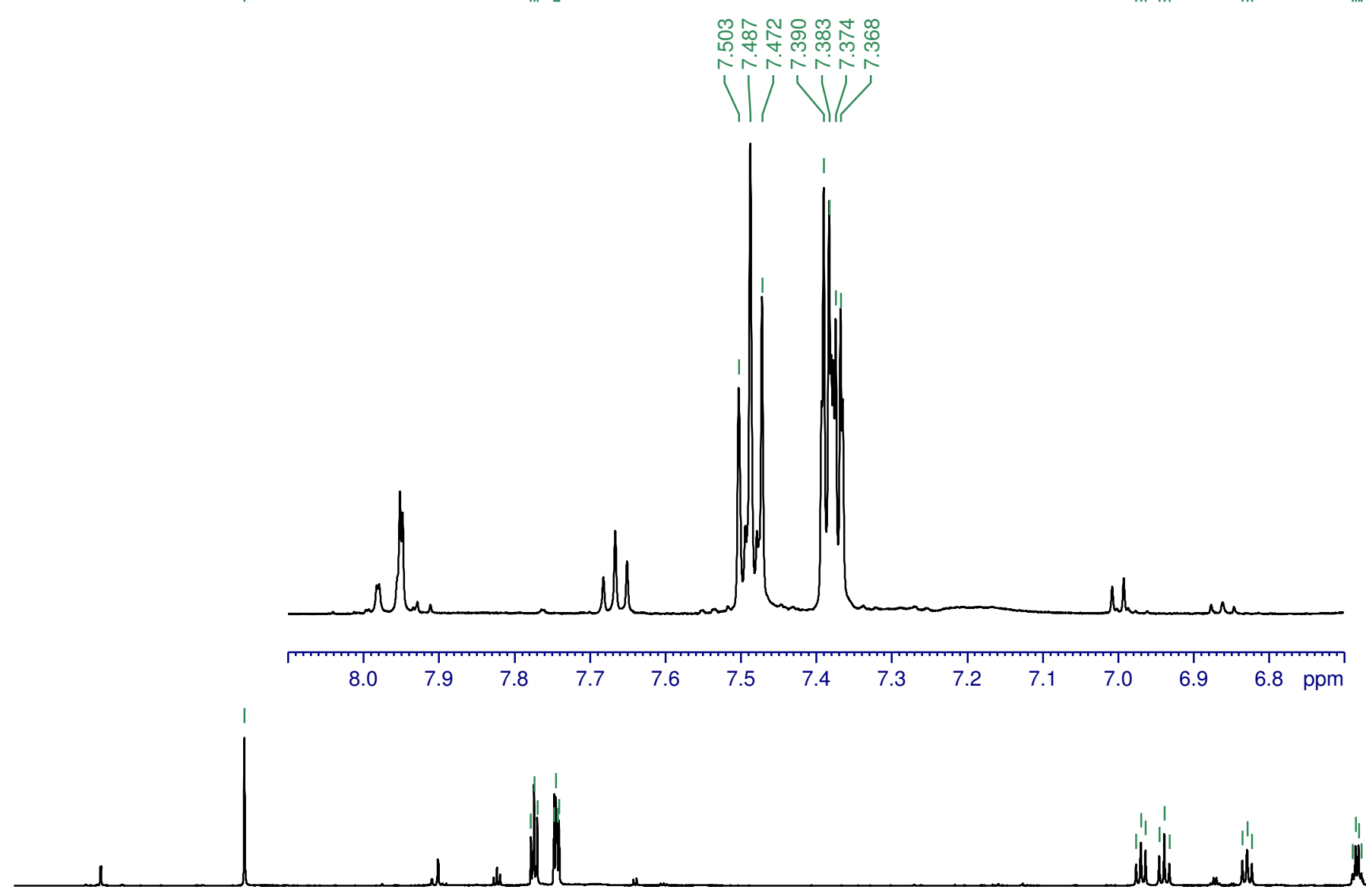

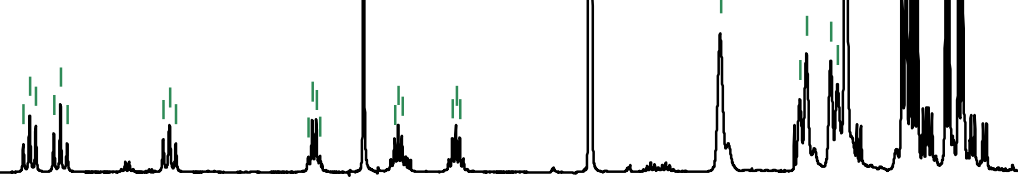

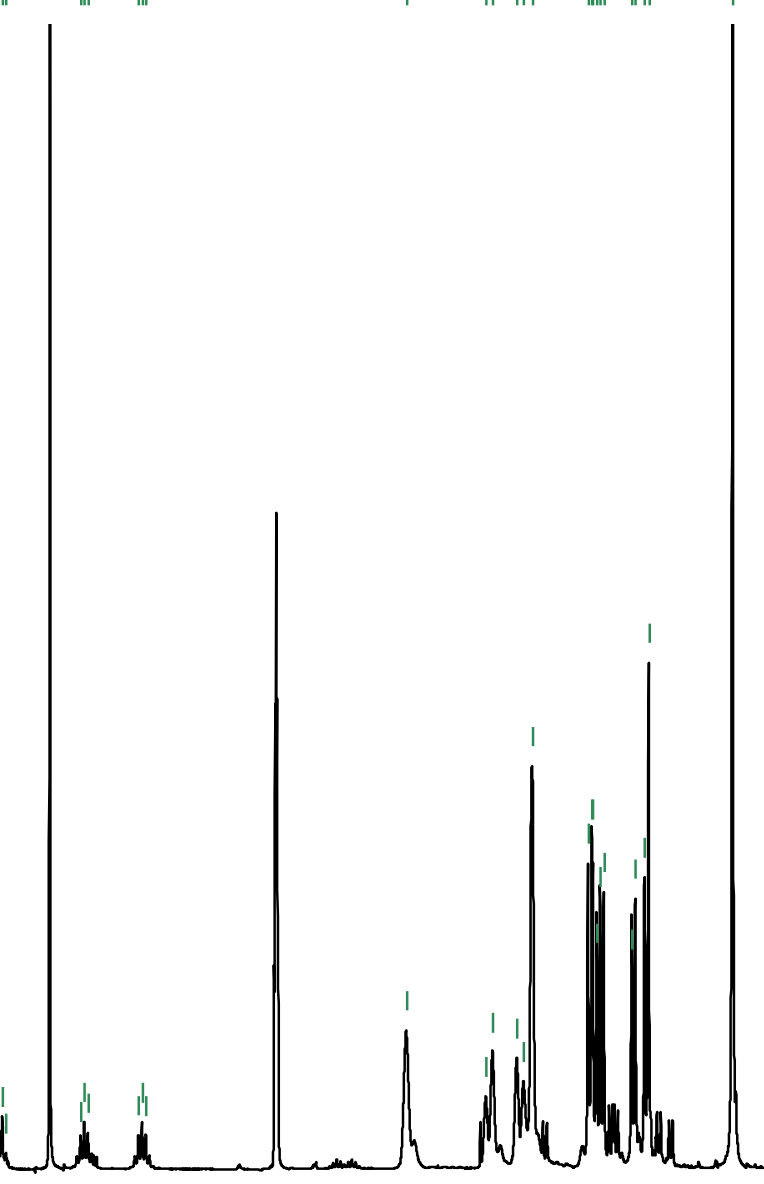

9.5
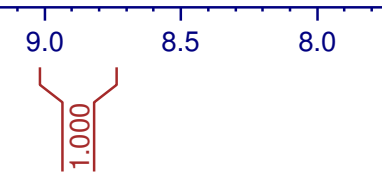

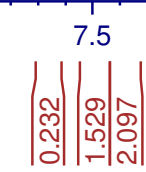

$\begin{array}{llll}7.0 & 6.5 & 6.0 & 5.5\end{array}$

5.0

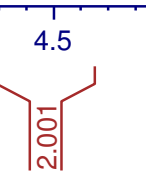

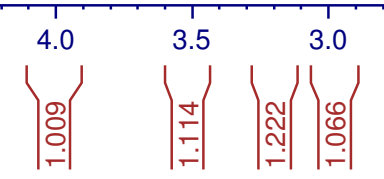

2.5

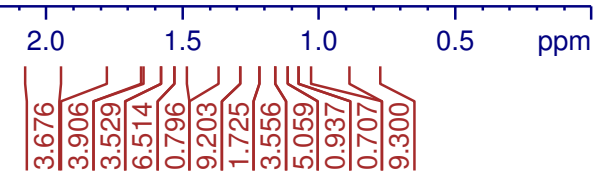



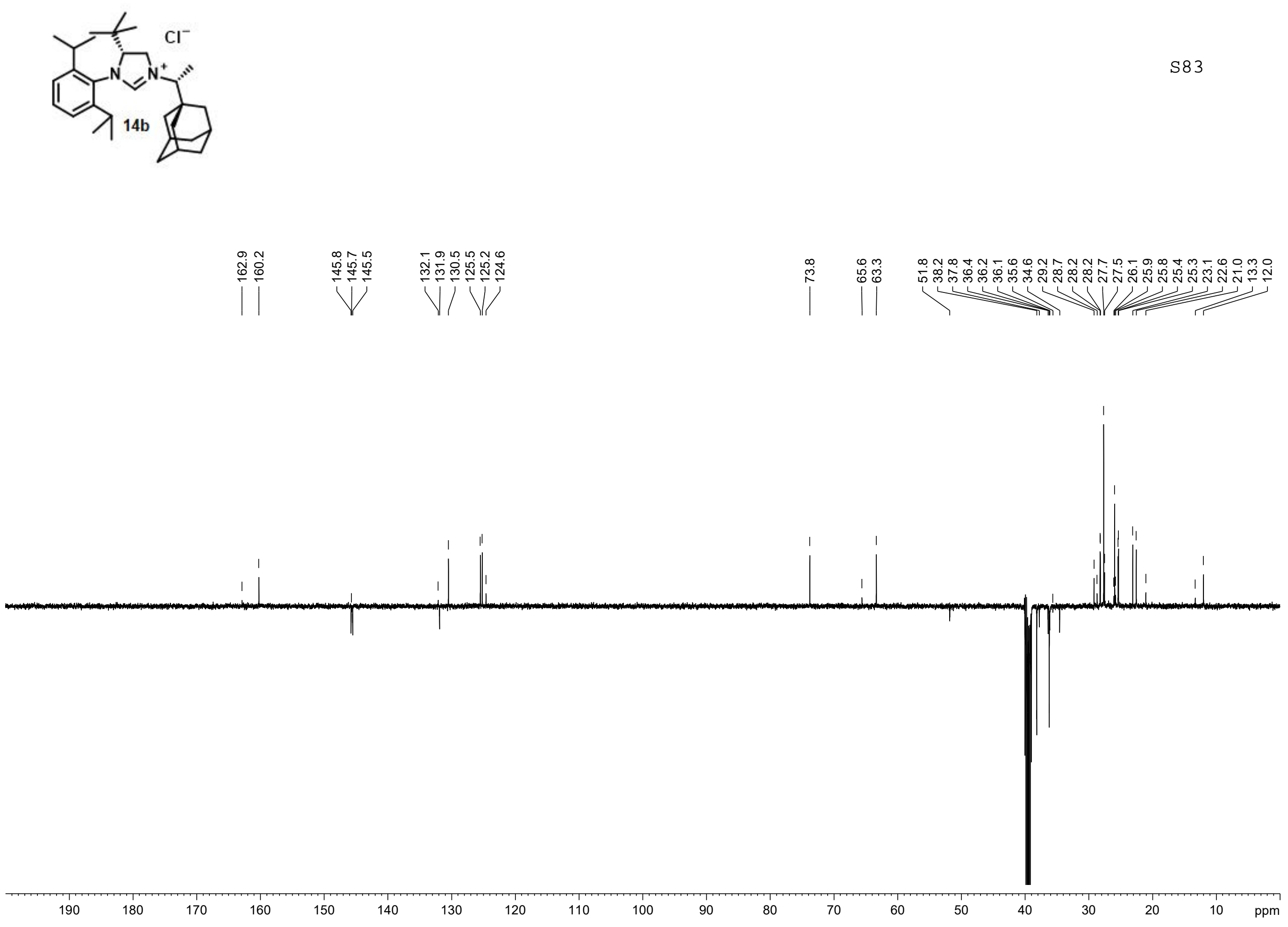

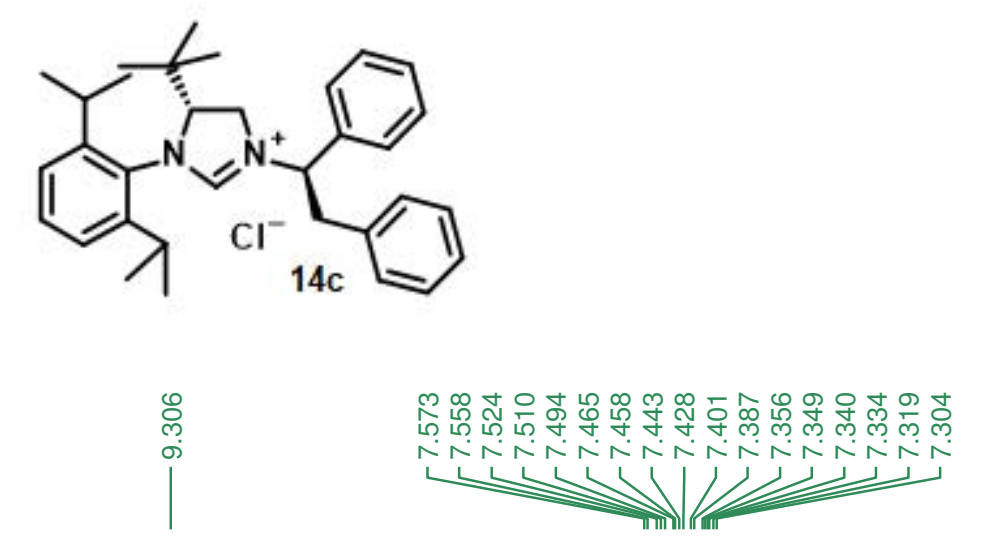

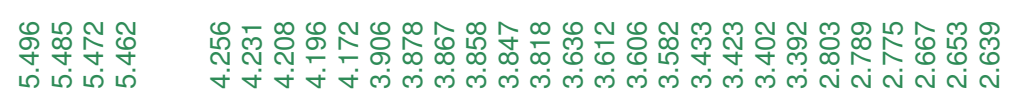



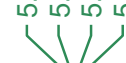

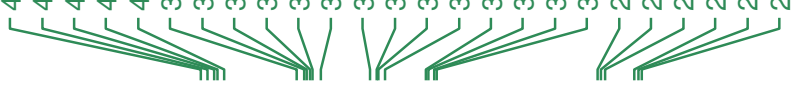

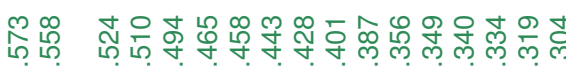

)

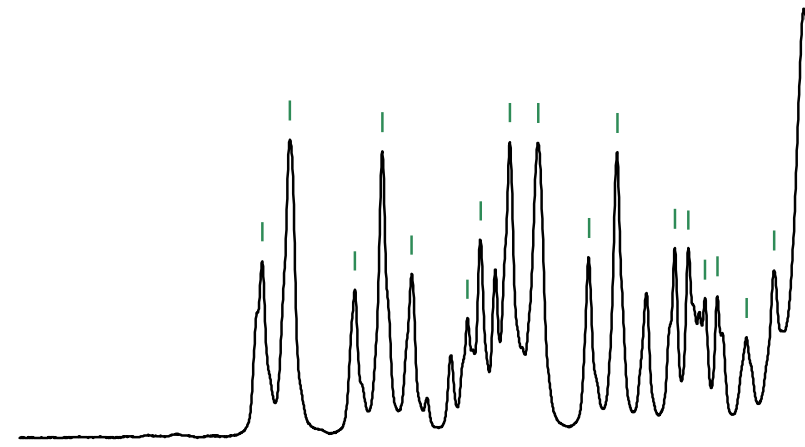

$\begin{array}{lllllllllll}7.65 & 7.60 & 7.55 & 7.50 & 7.45 & 7.40 & 7.35 & 7.30 & 7.25 & 7.20 & 7.15 \mathrm{ppm}\end{array}$

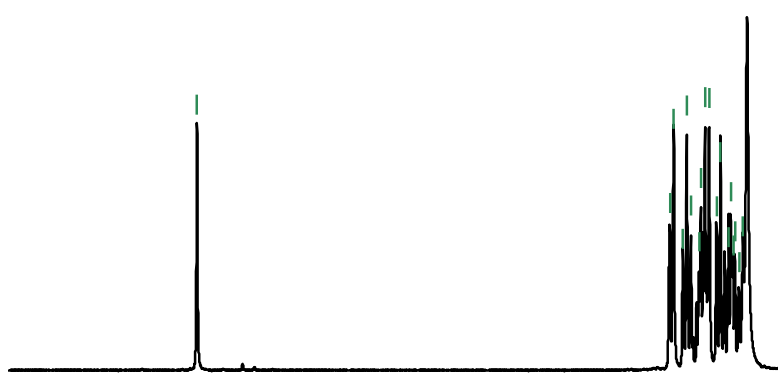

buth bill
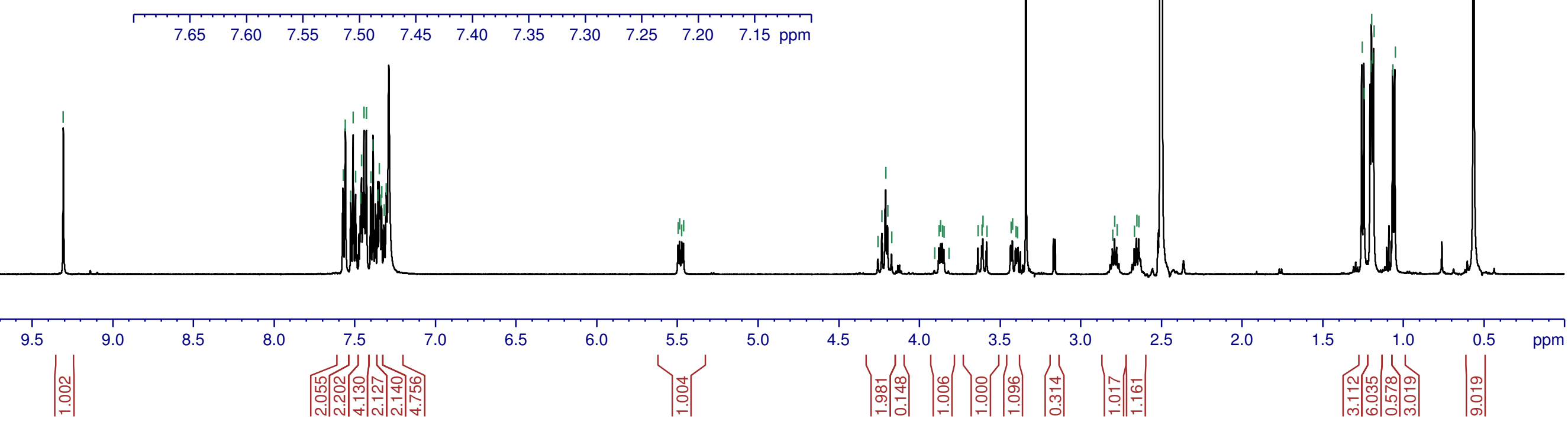

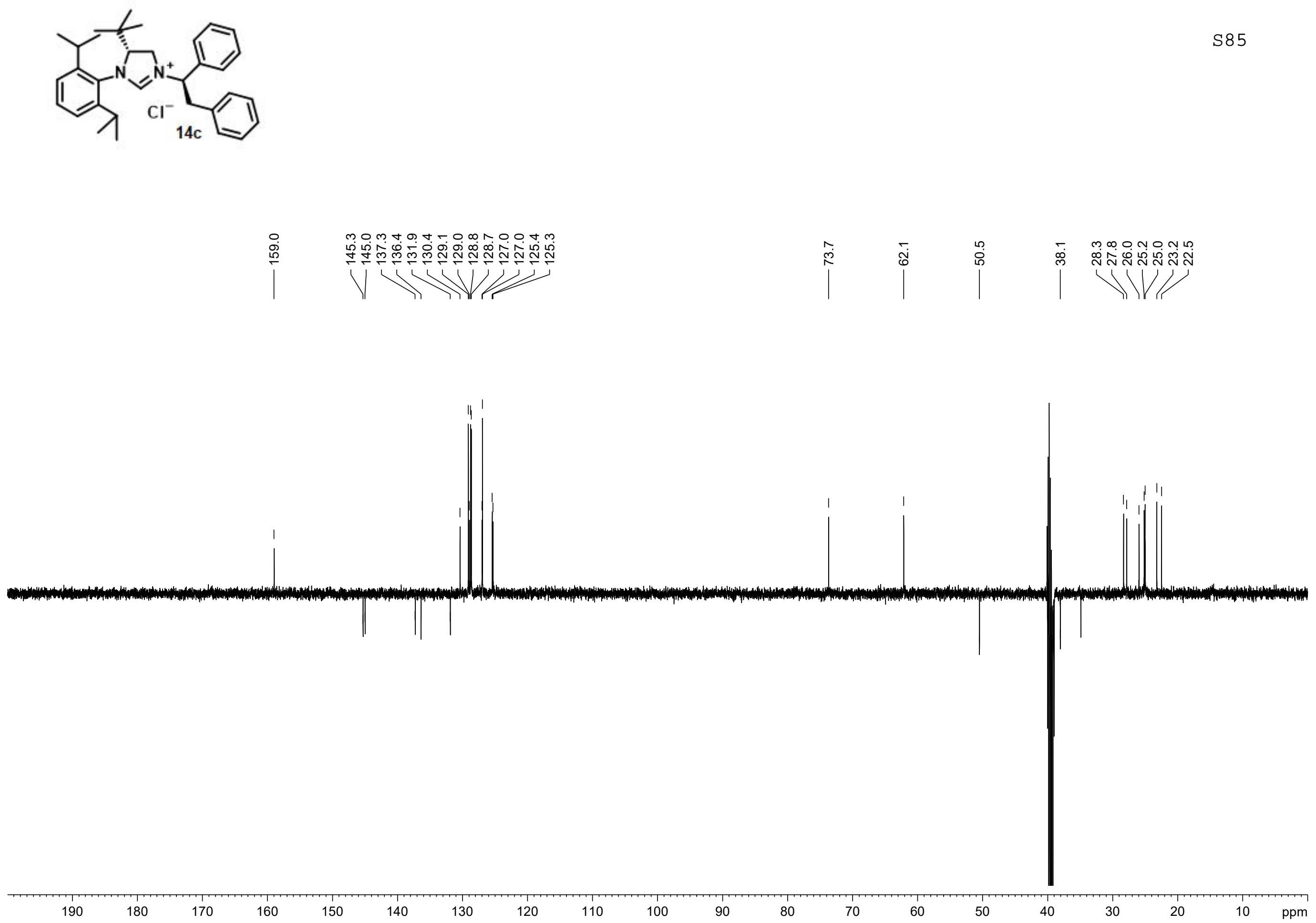

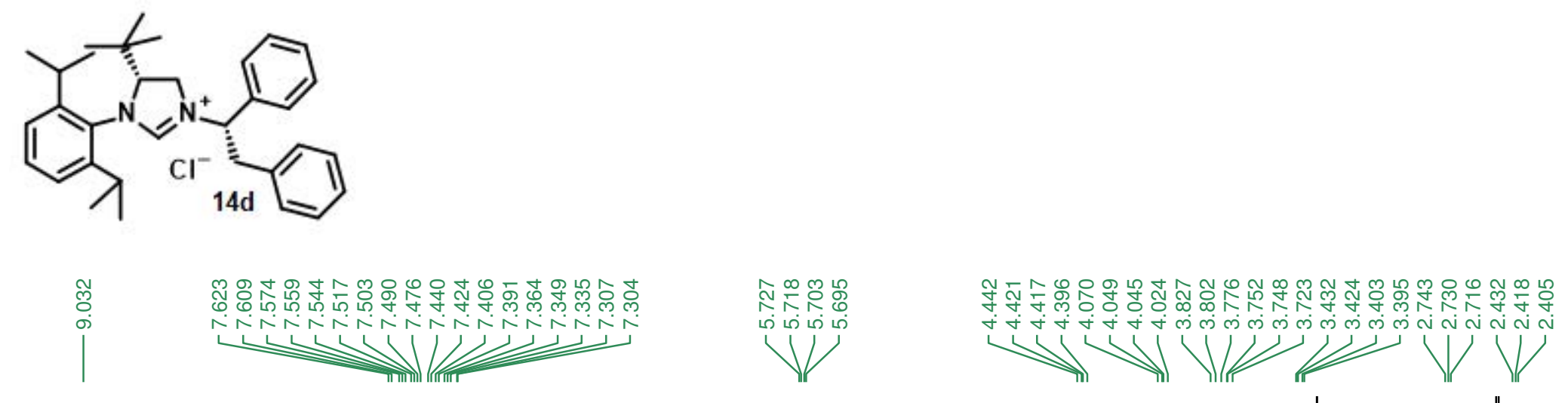

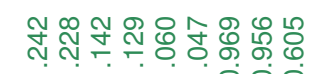

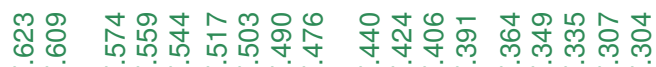

li
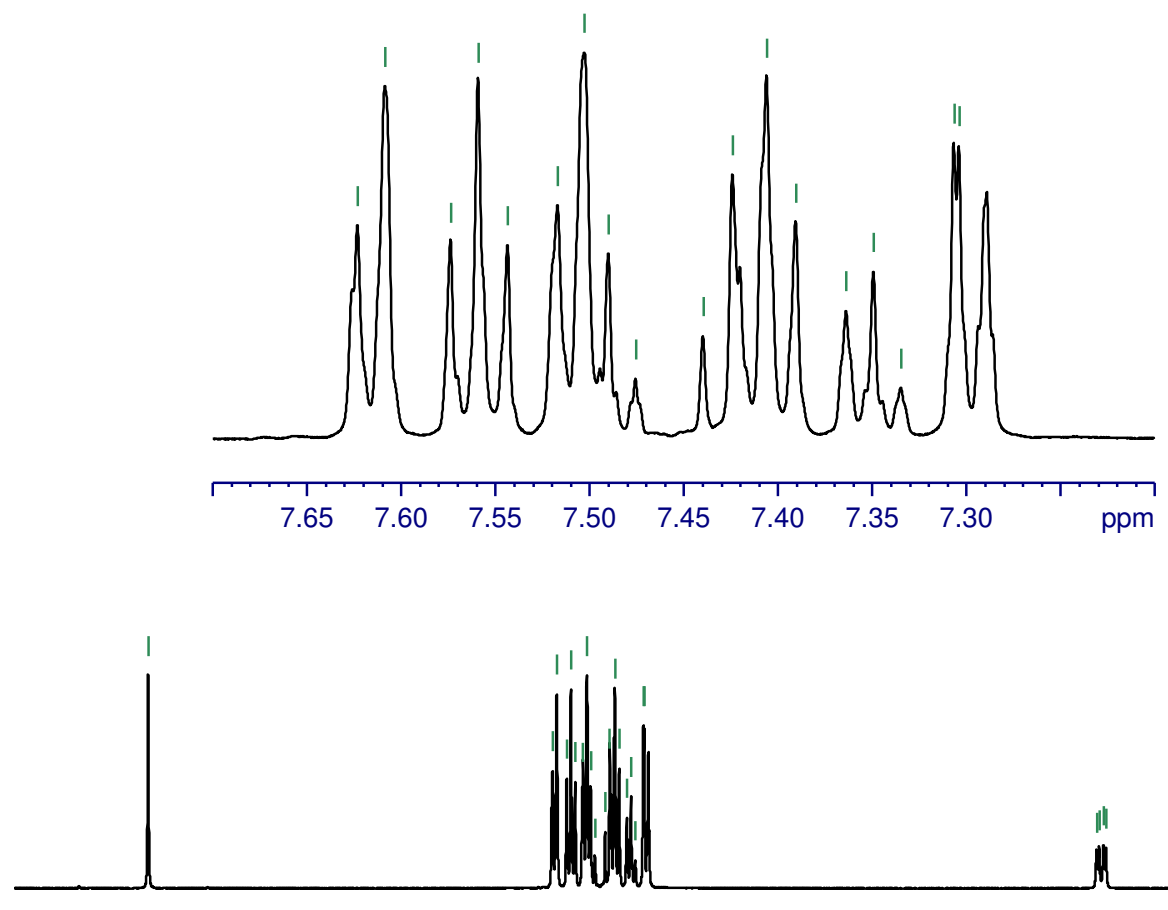

$\mathrm{ppm}$





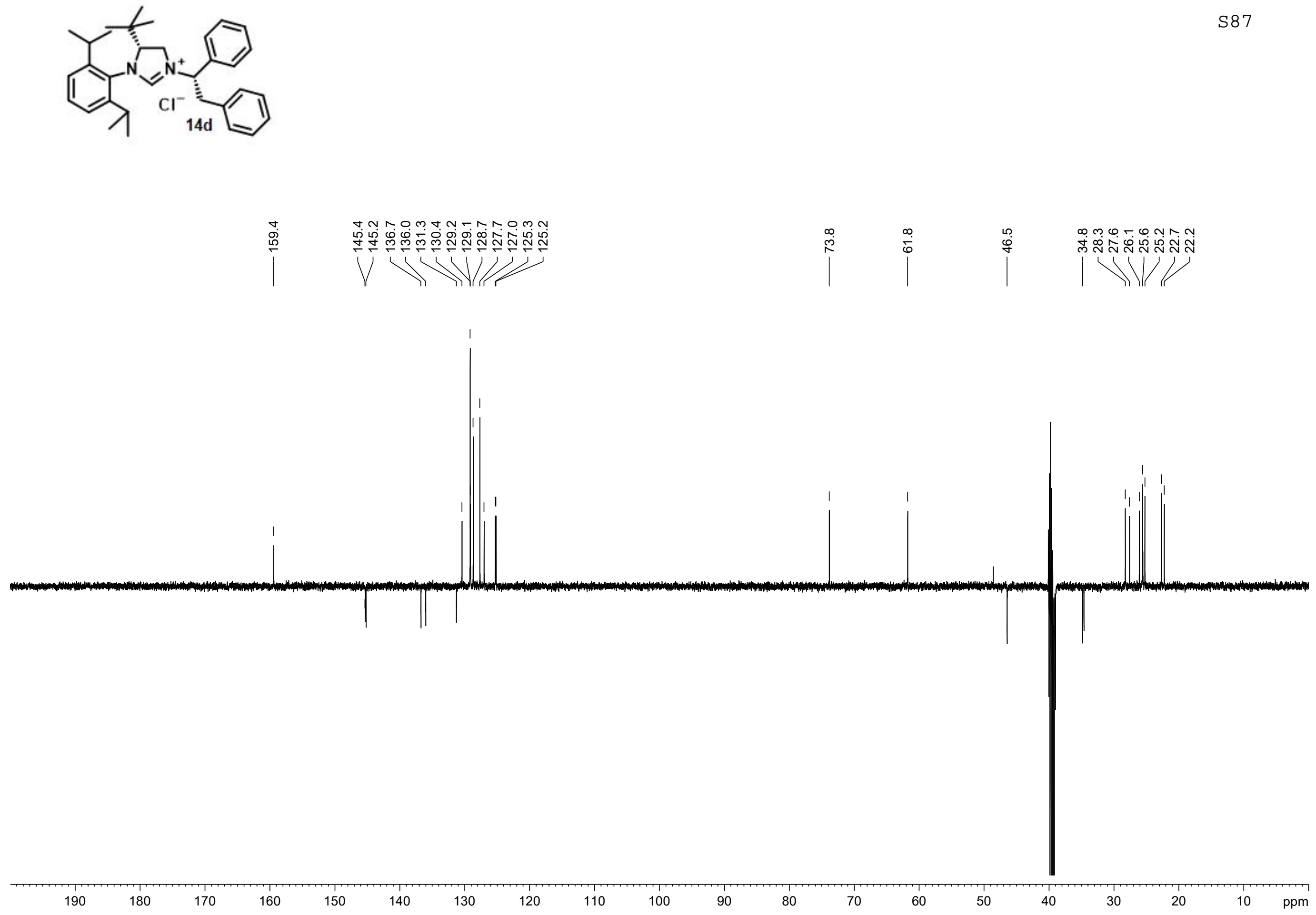

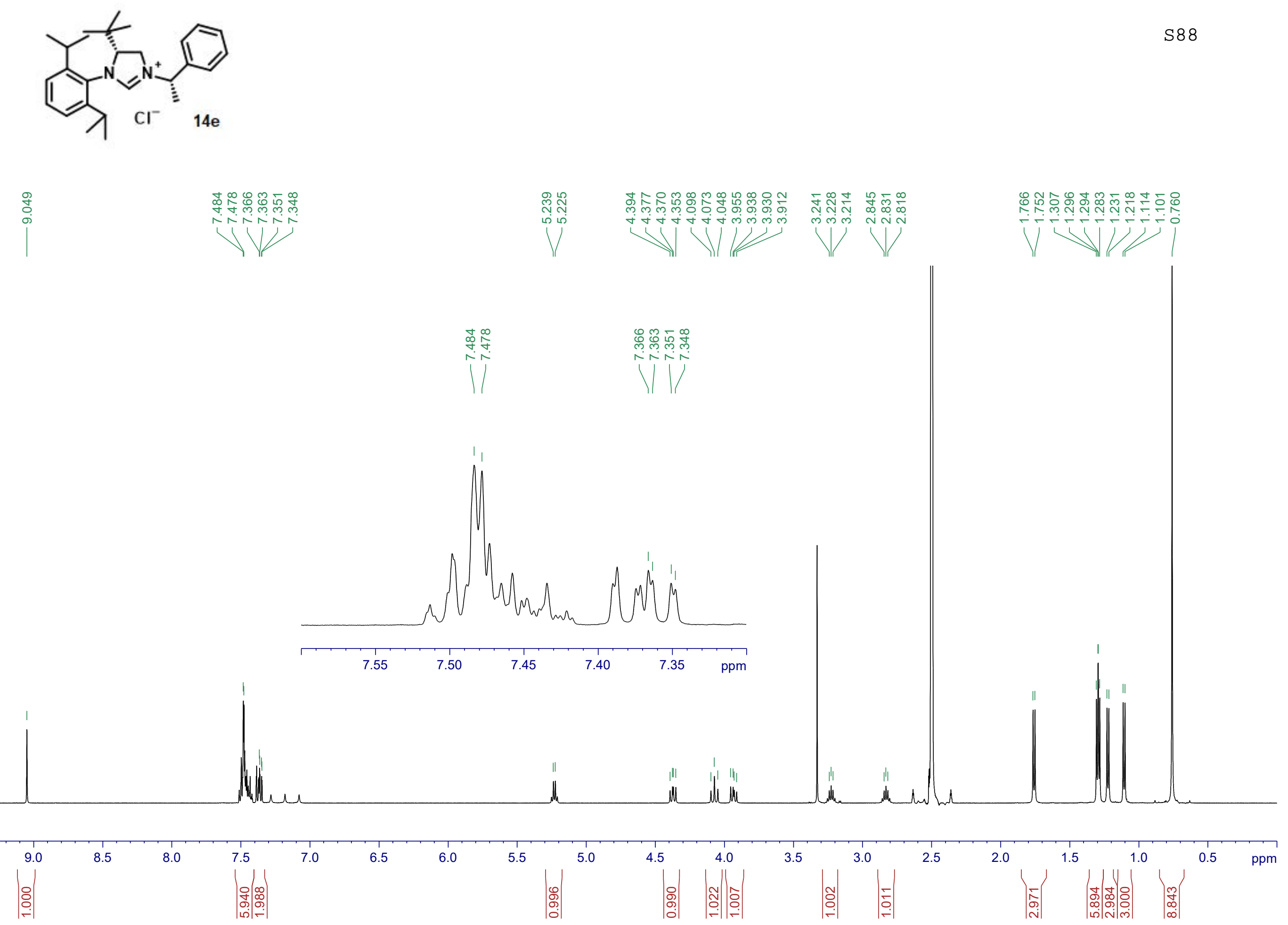

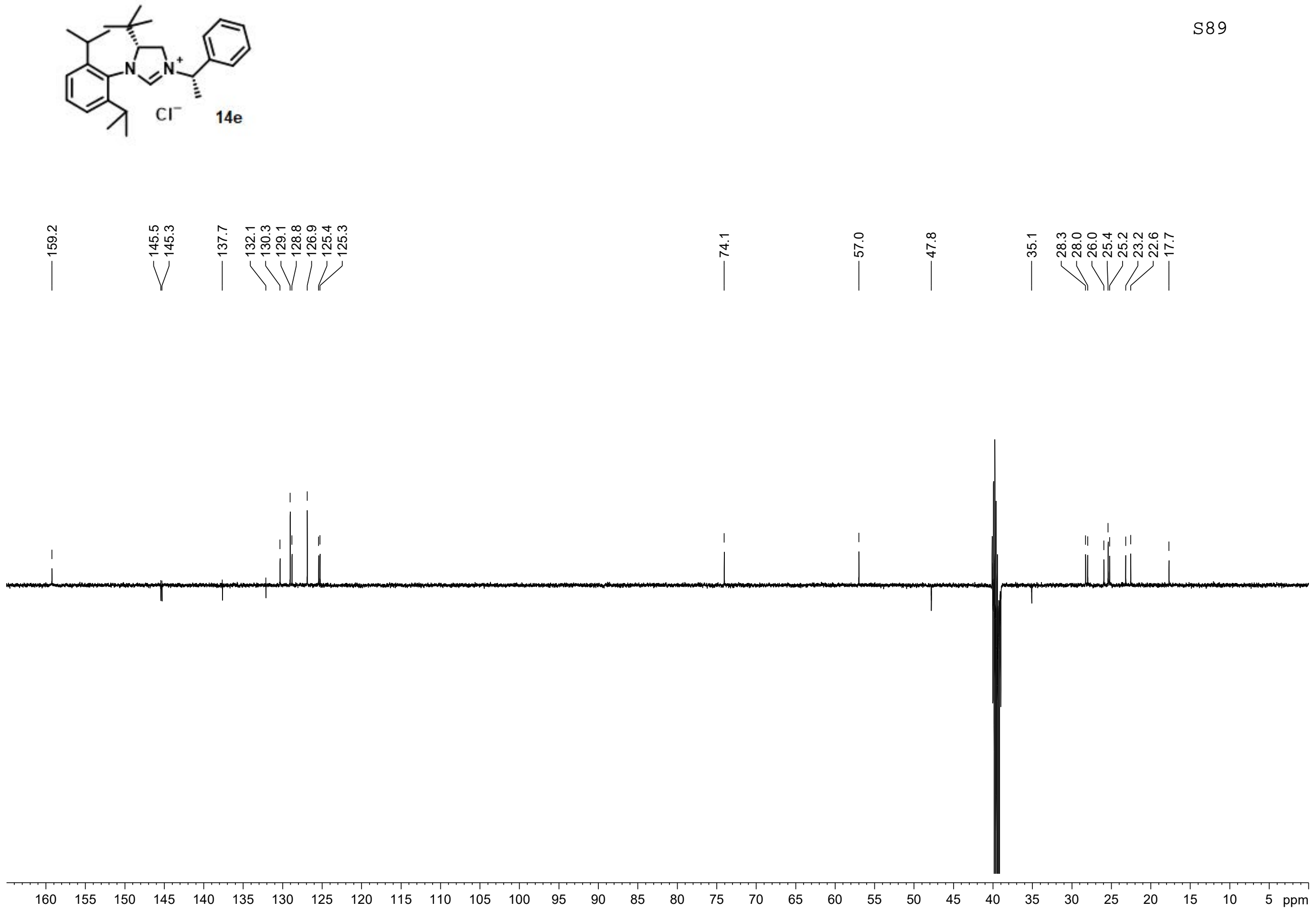

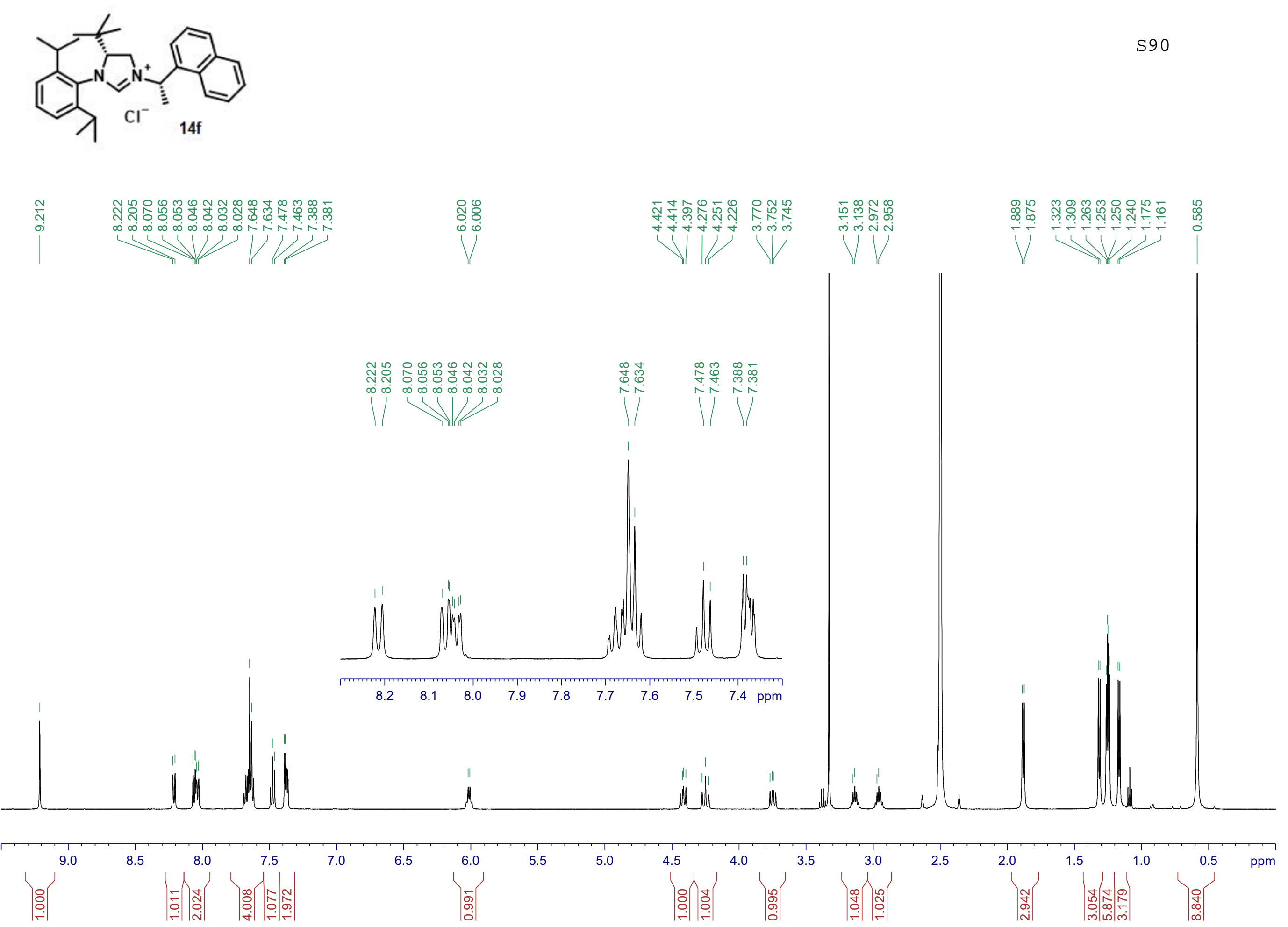

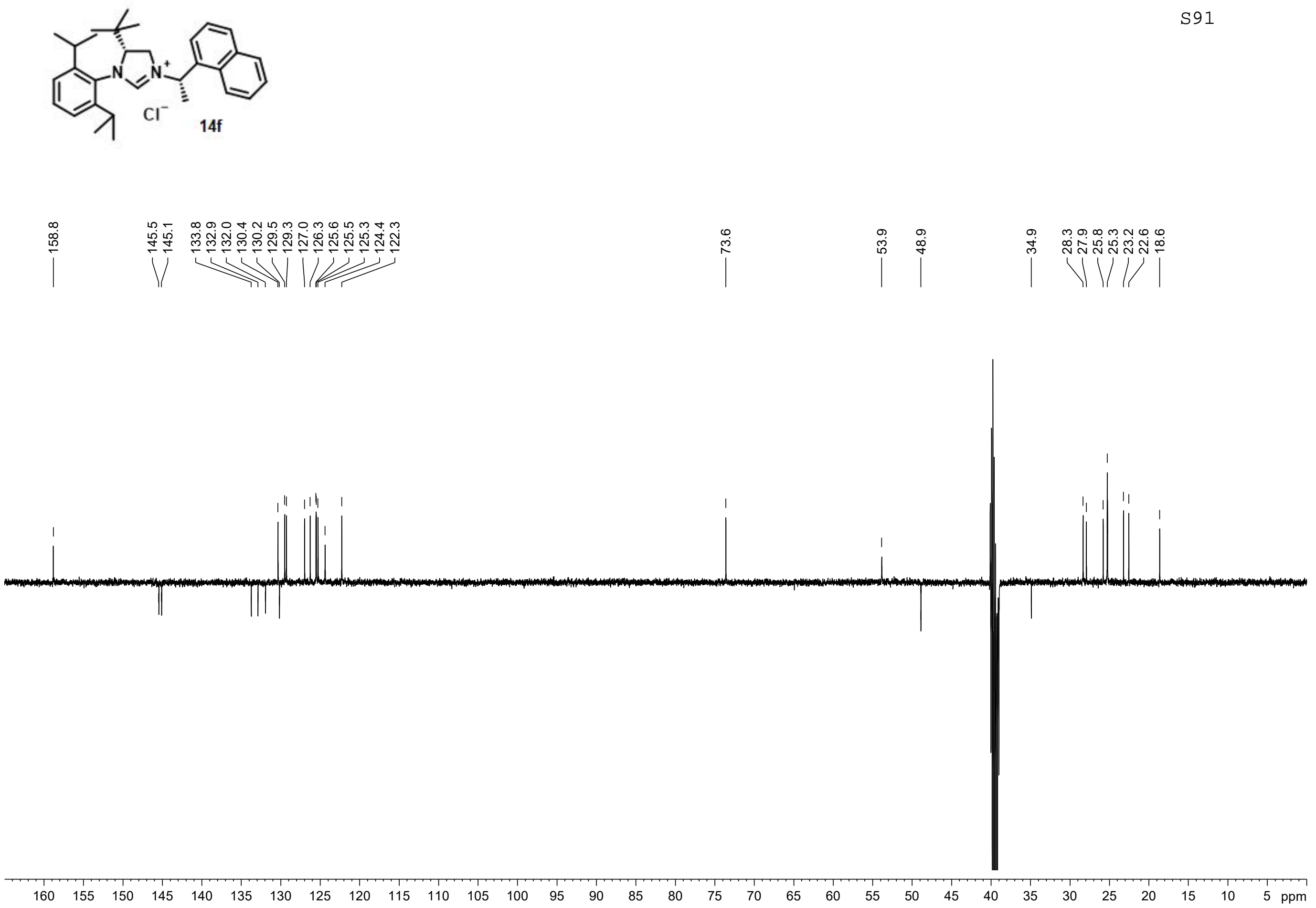

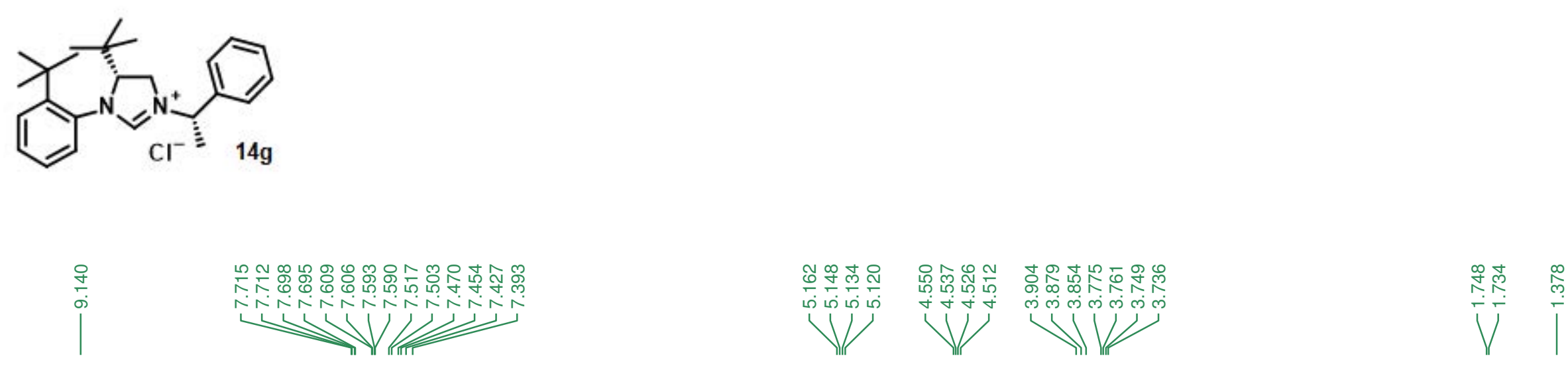

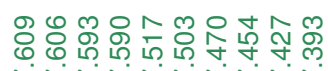

UNillll

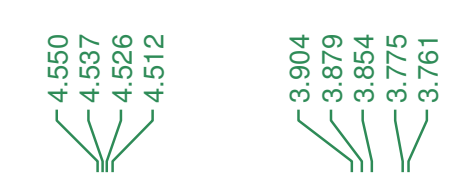

ith

แ่

पiV

7.5

7.0

6.5

6.0

5.5

5.0

ith

wh
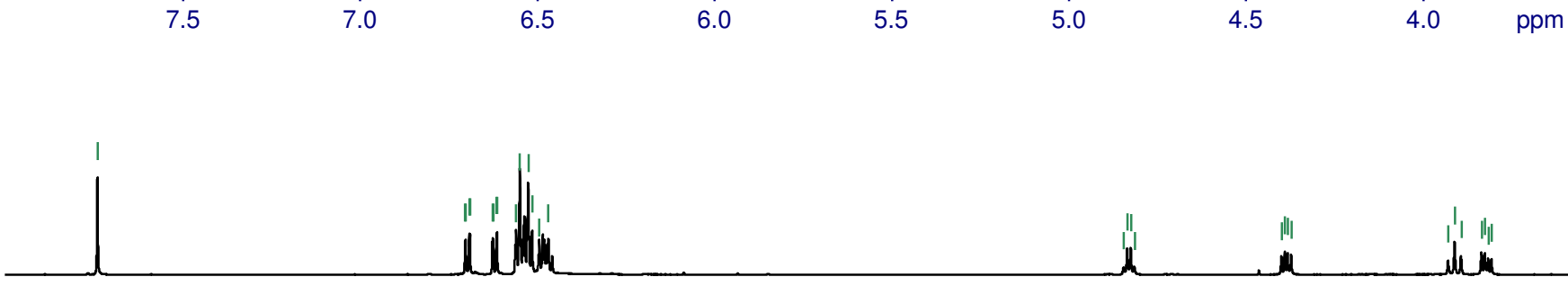

i
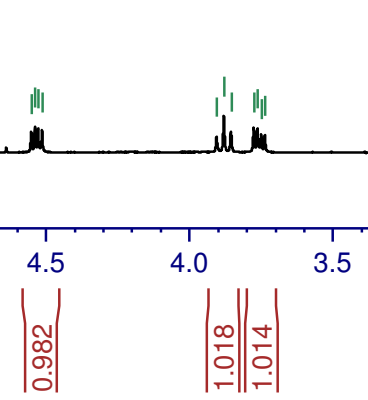

$3.5 \quad 3.0 \quad 2.5$

|ํ.

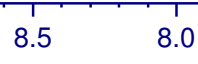

|

$7.0 \quad 6.5$

$6.0 \quad 5.5$

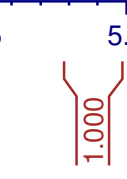

òำ

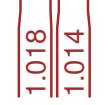

2.0

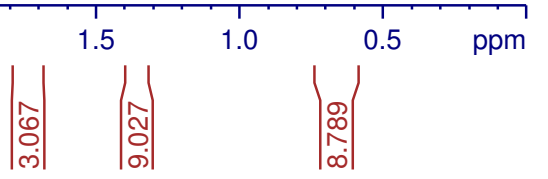



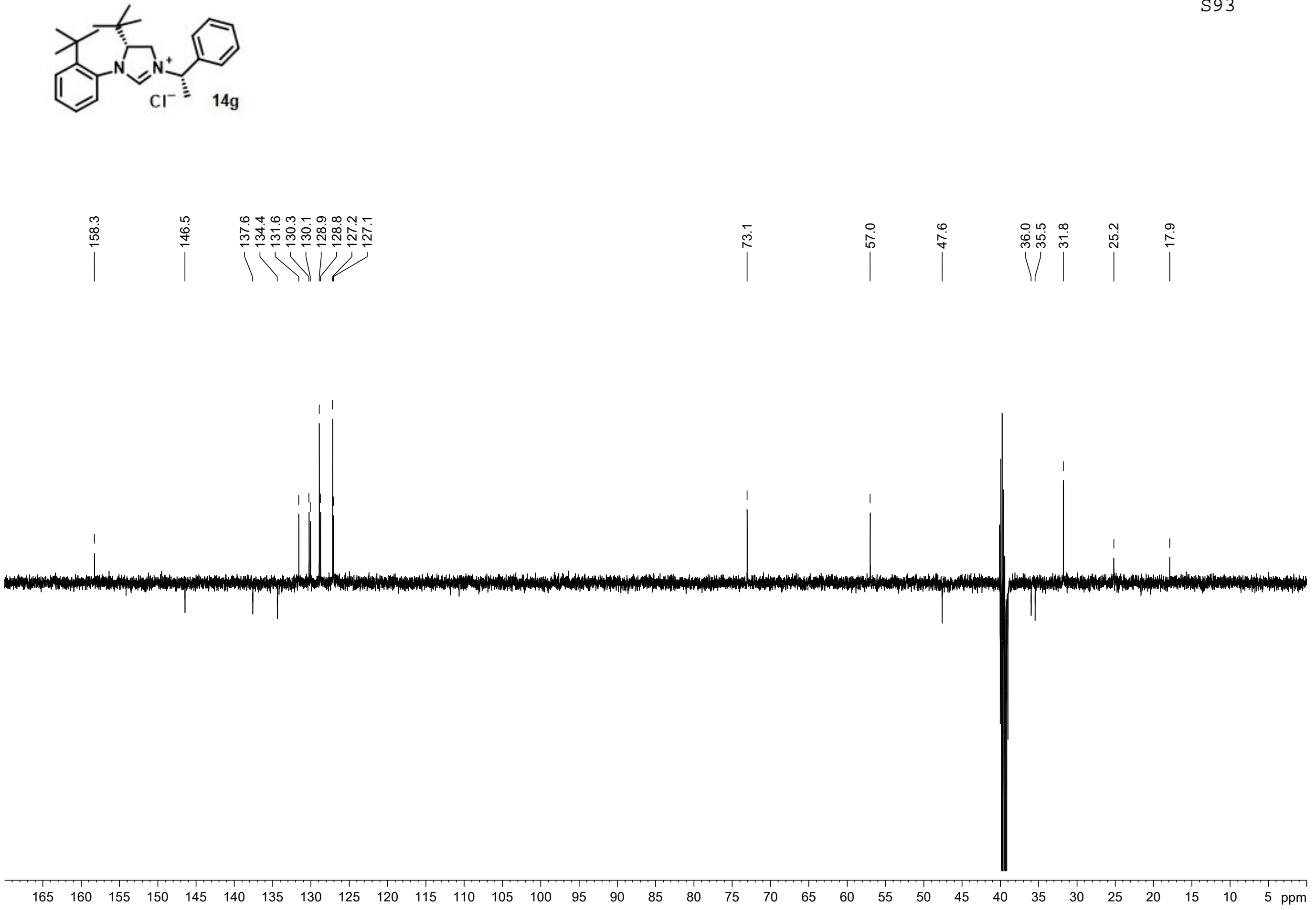




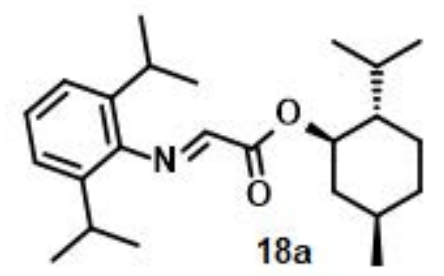

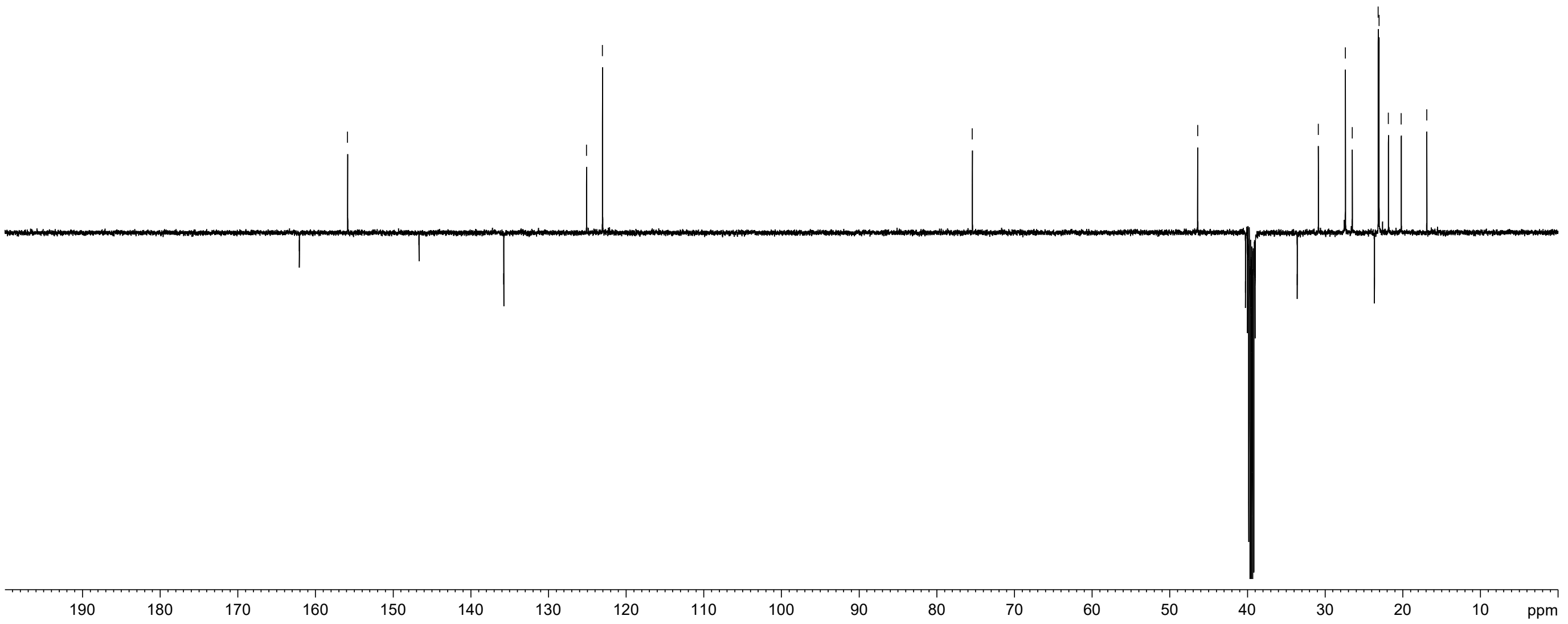



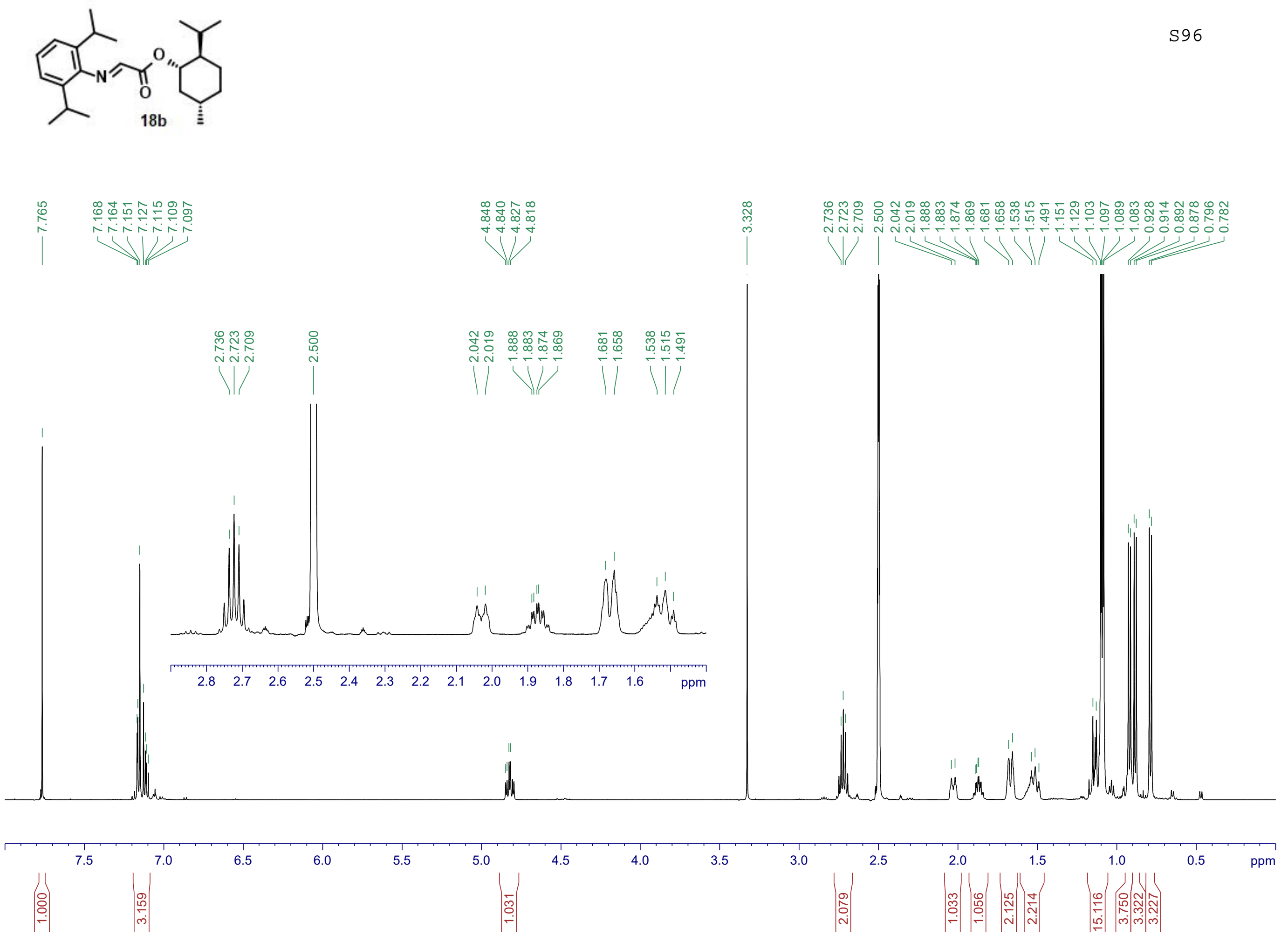


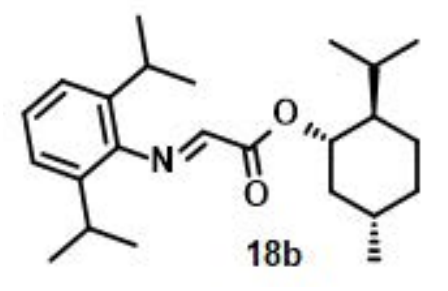

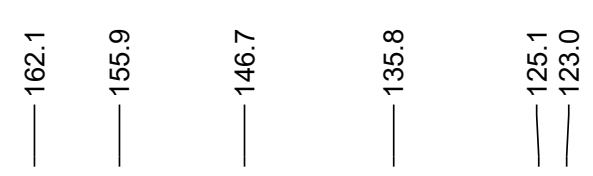

官

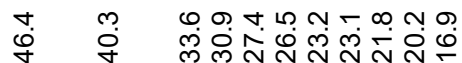

1111111

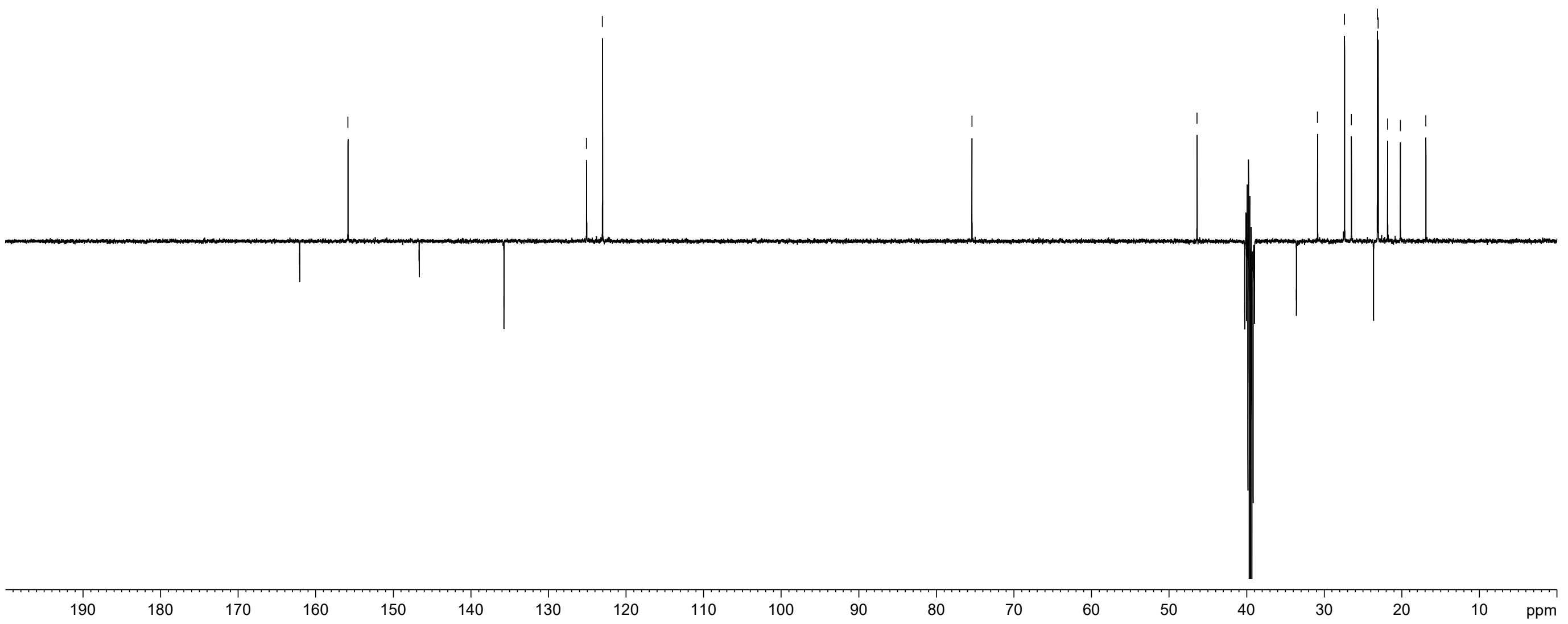



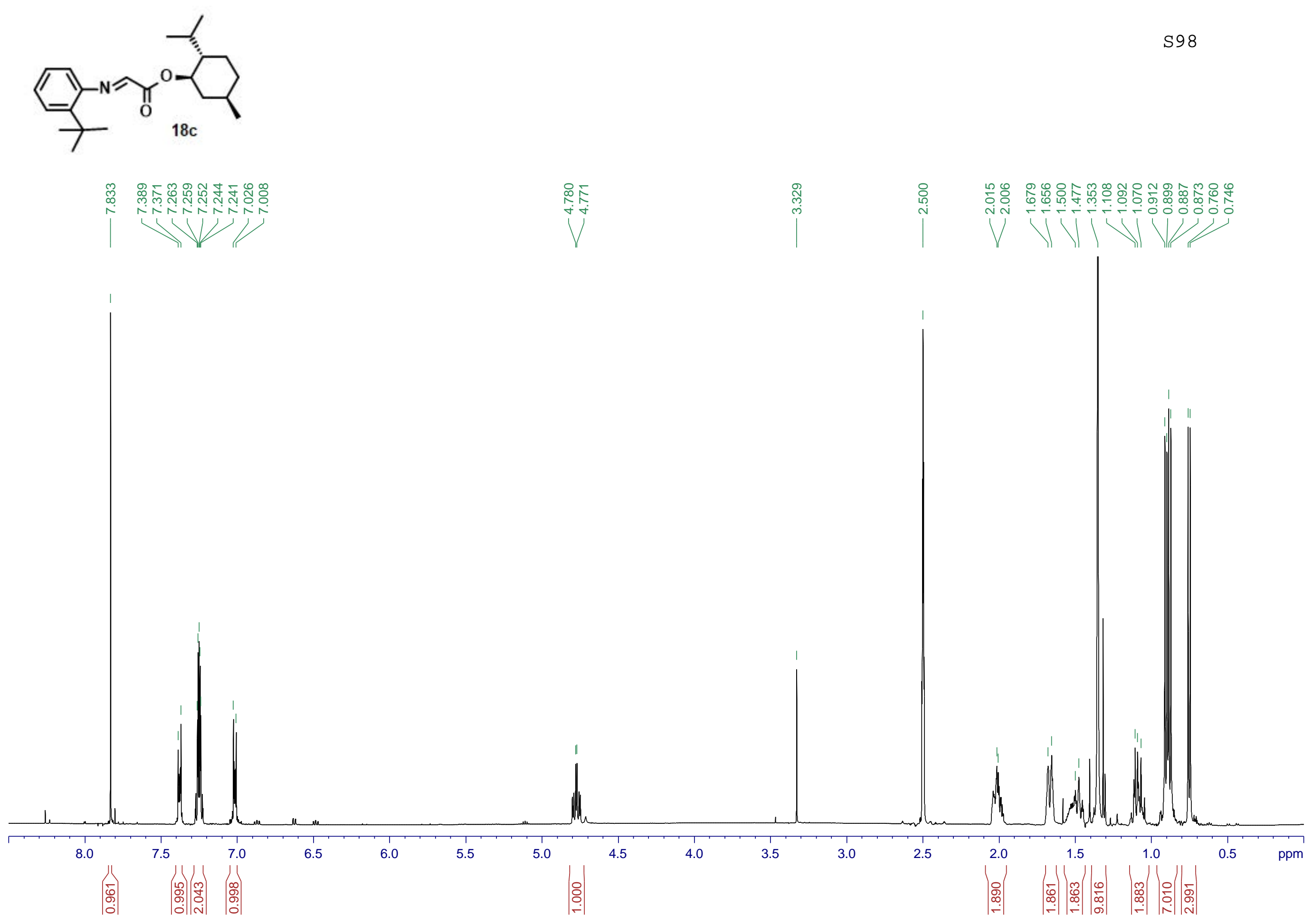

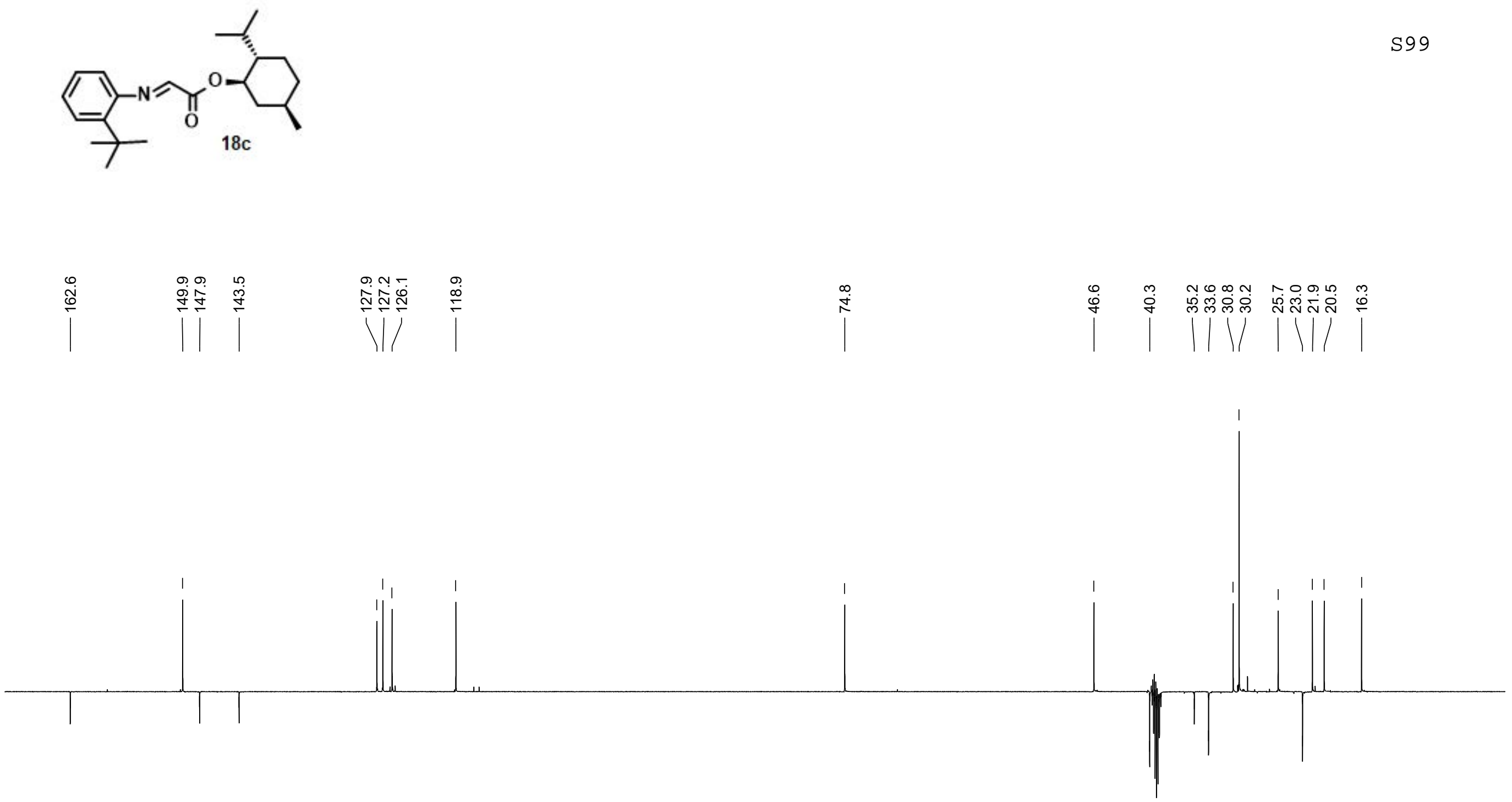

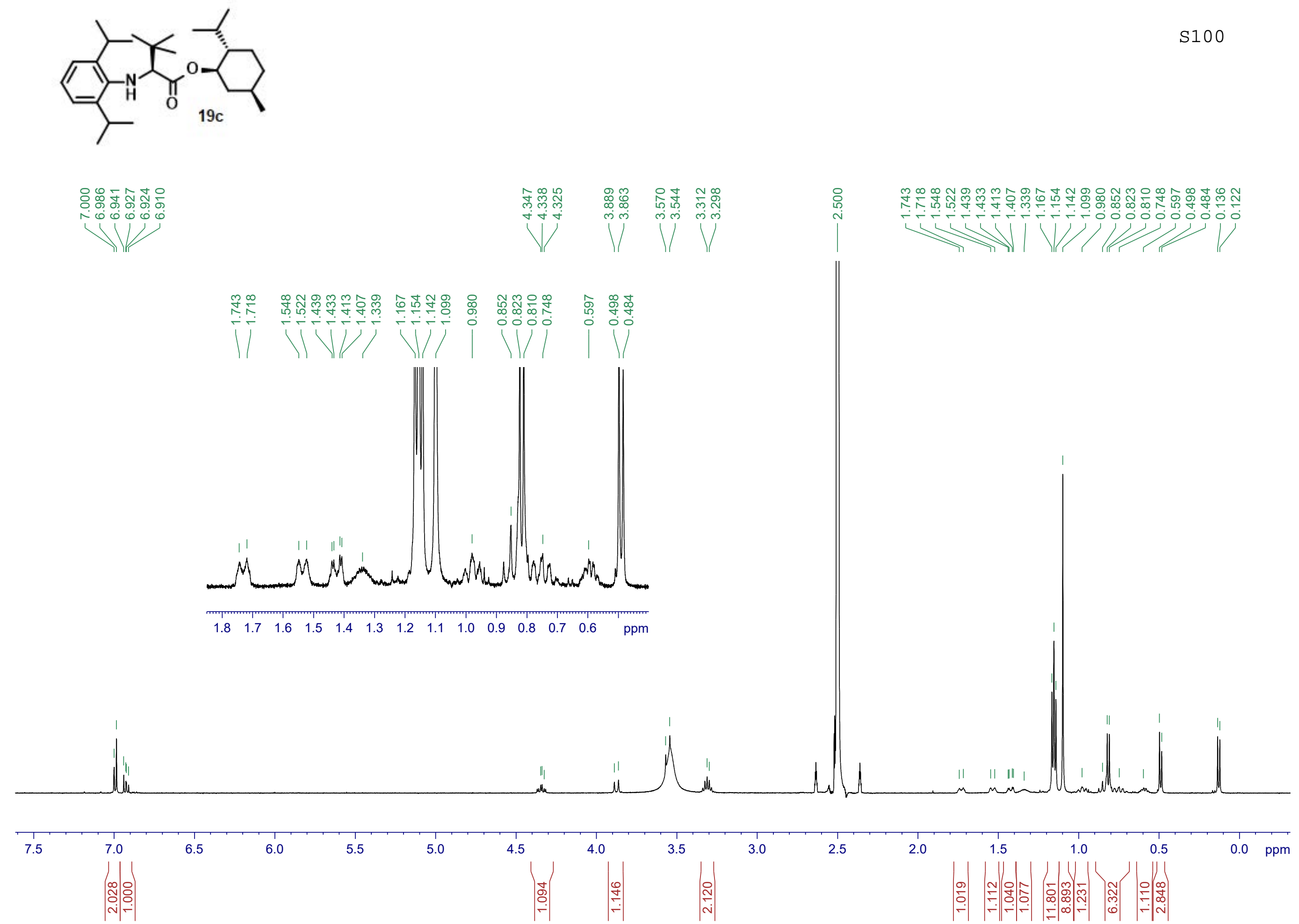
$=$

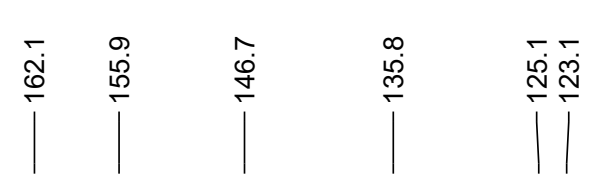

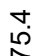

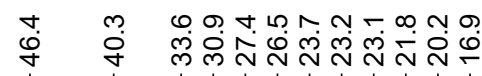

|

।

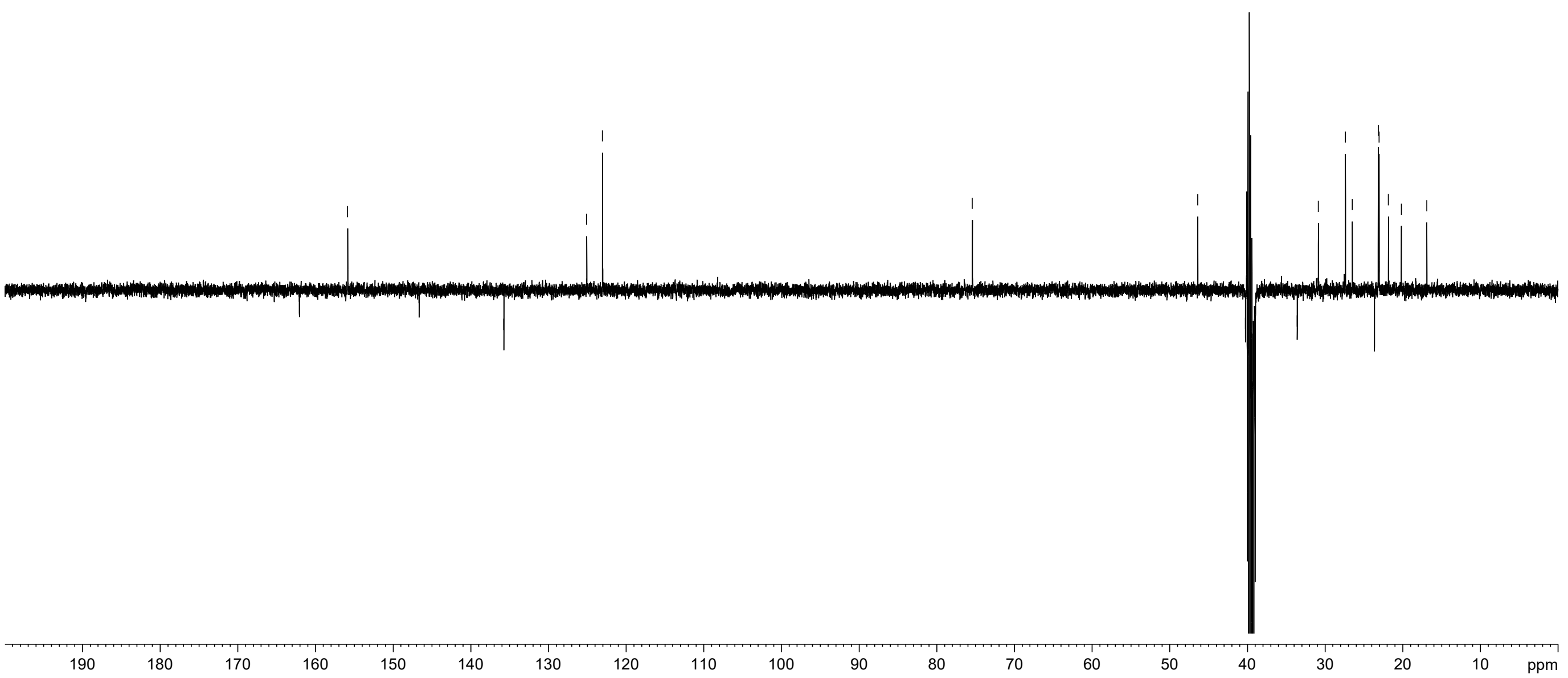



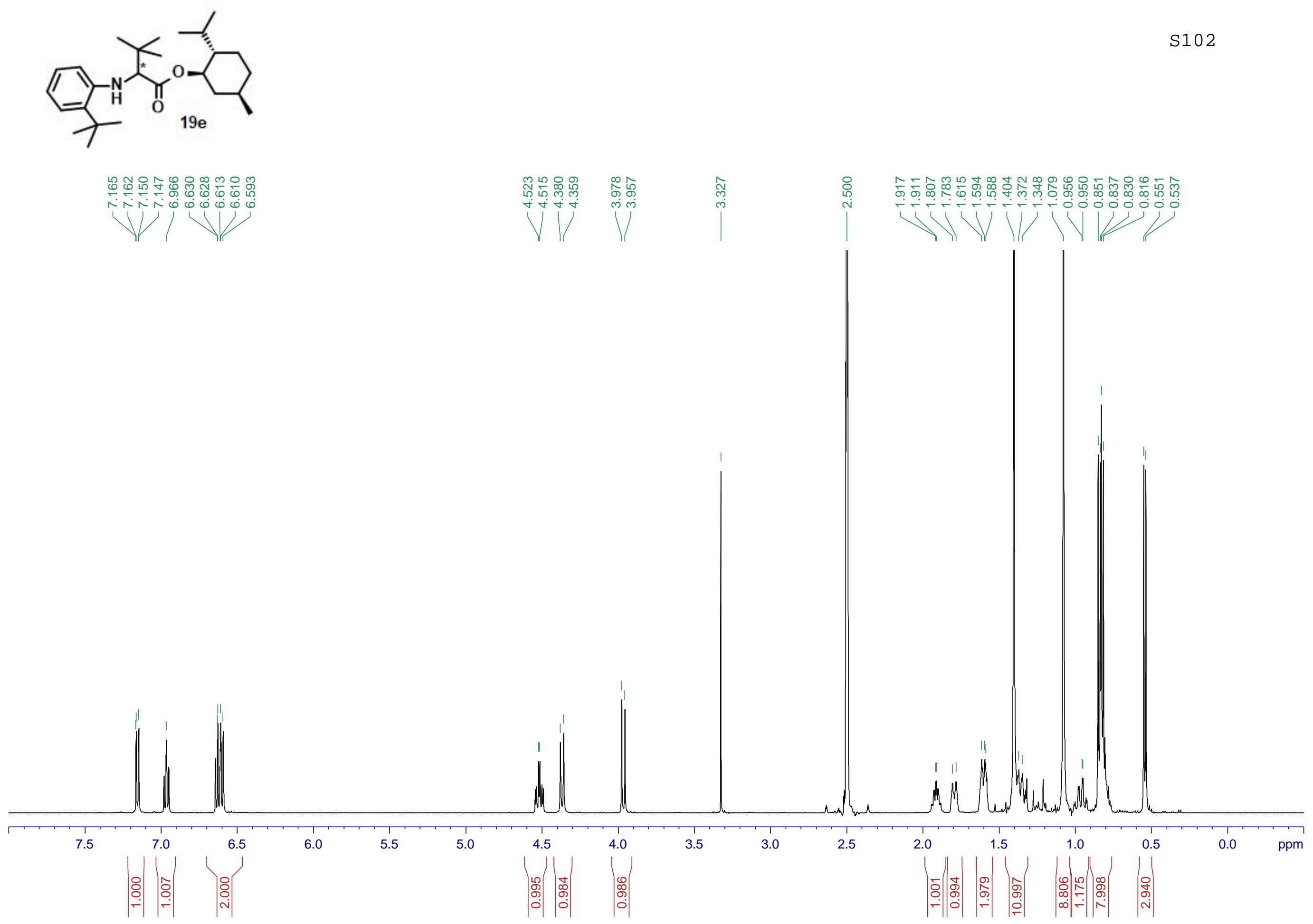


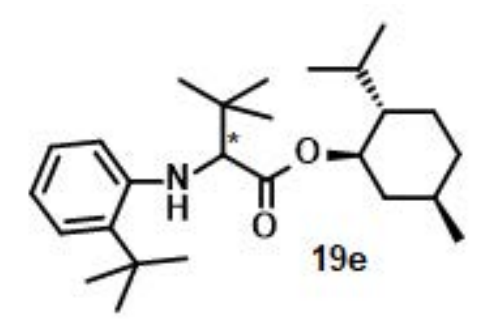

$\mid$

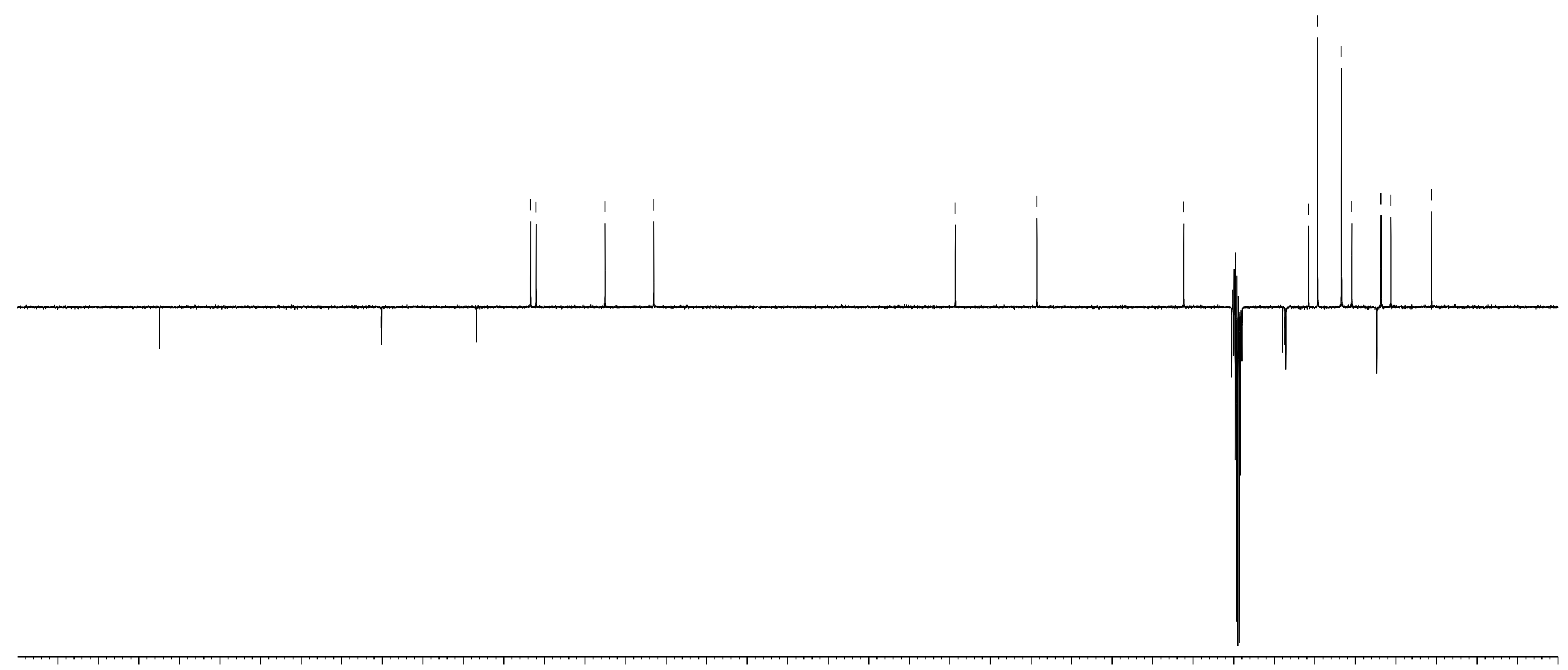




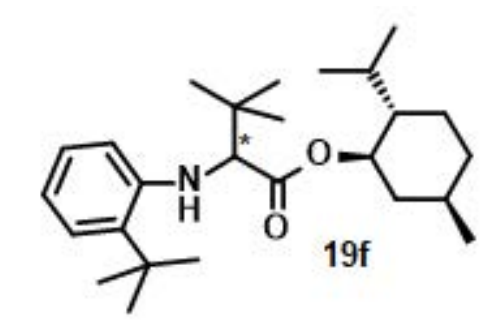

S104

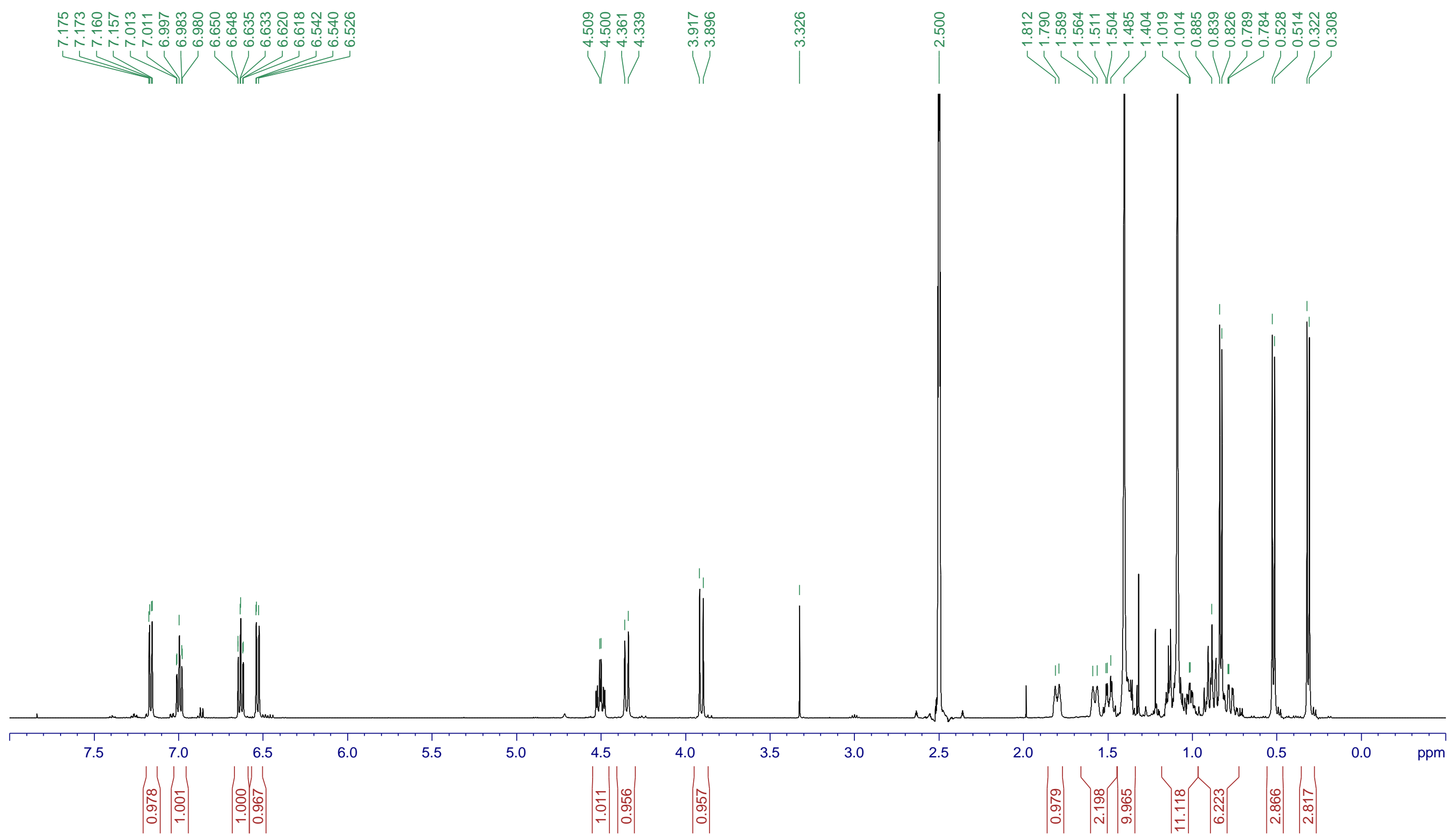




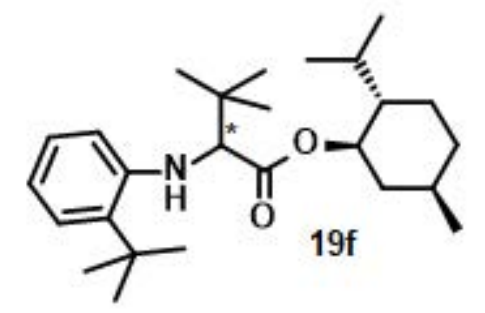

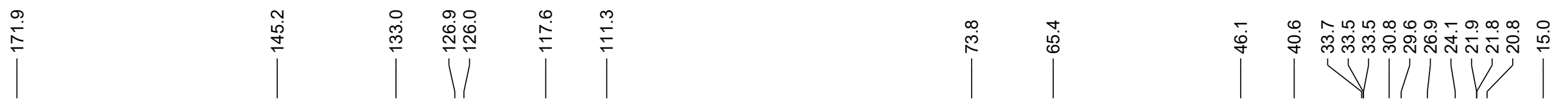







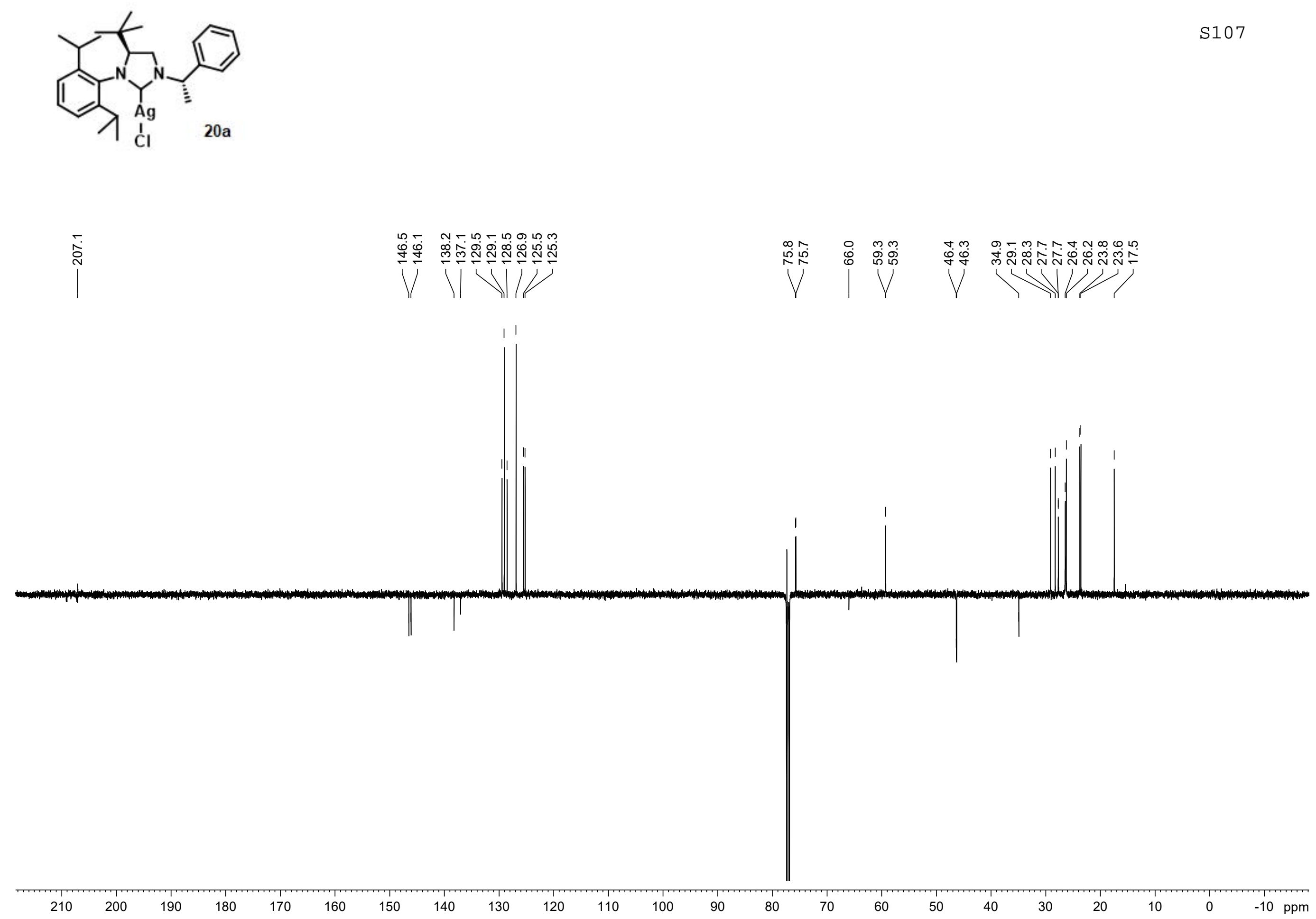

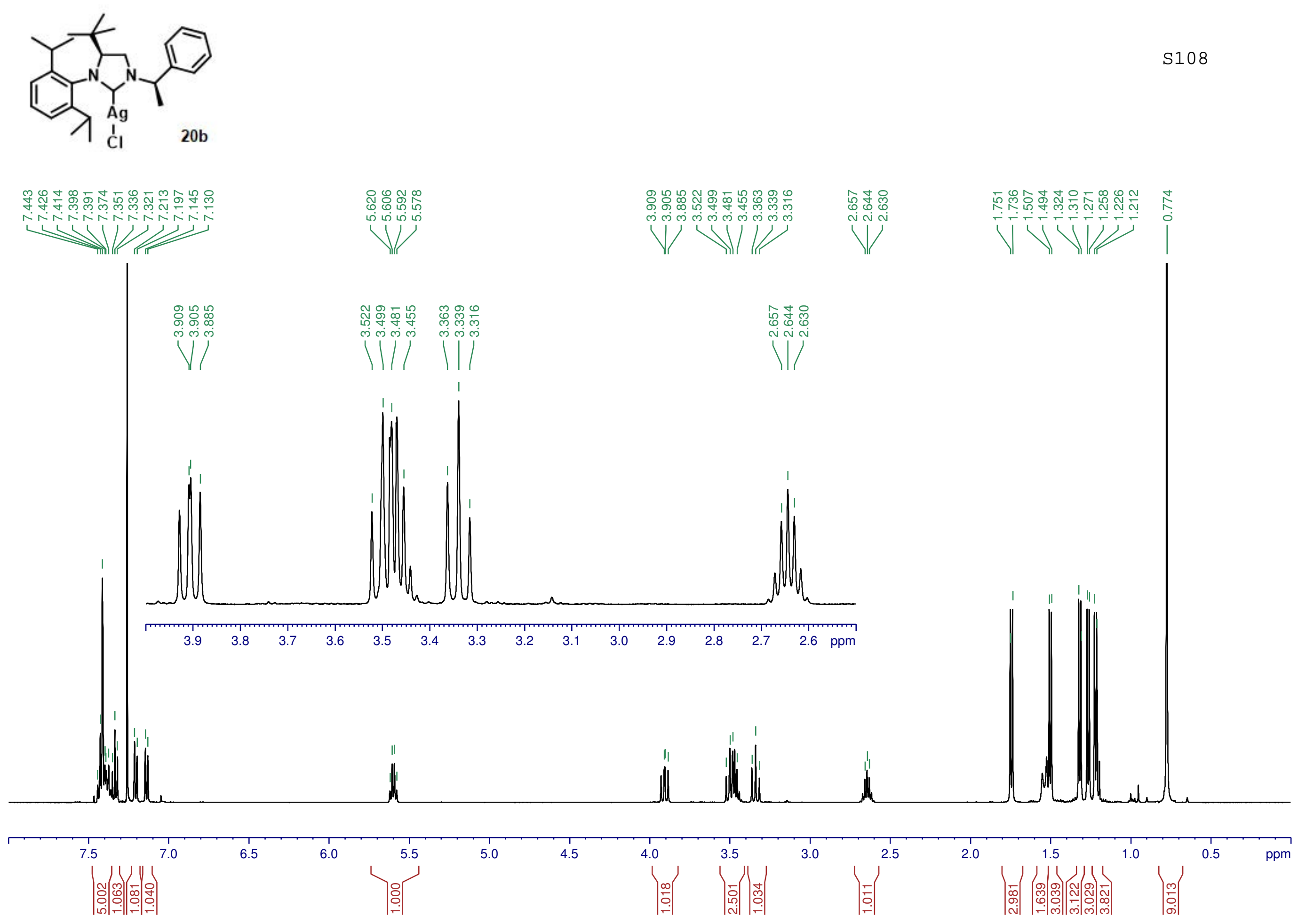

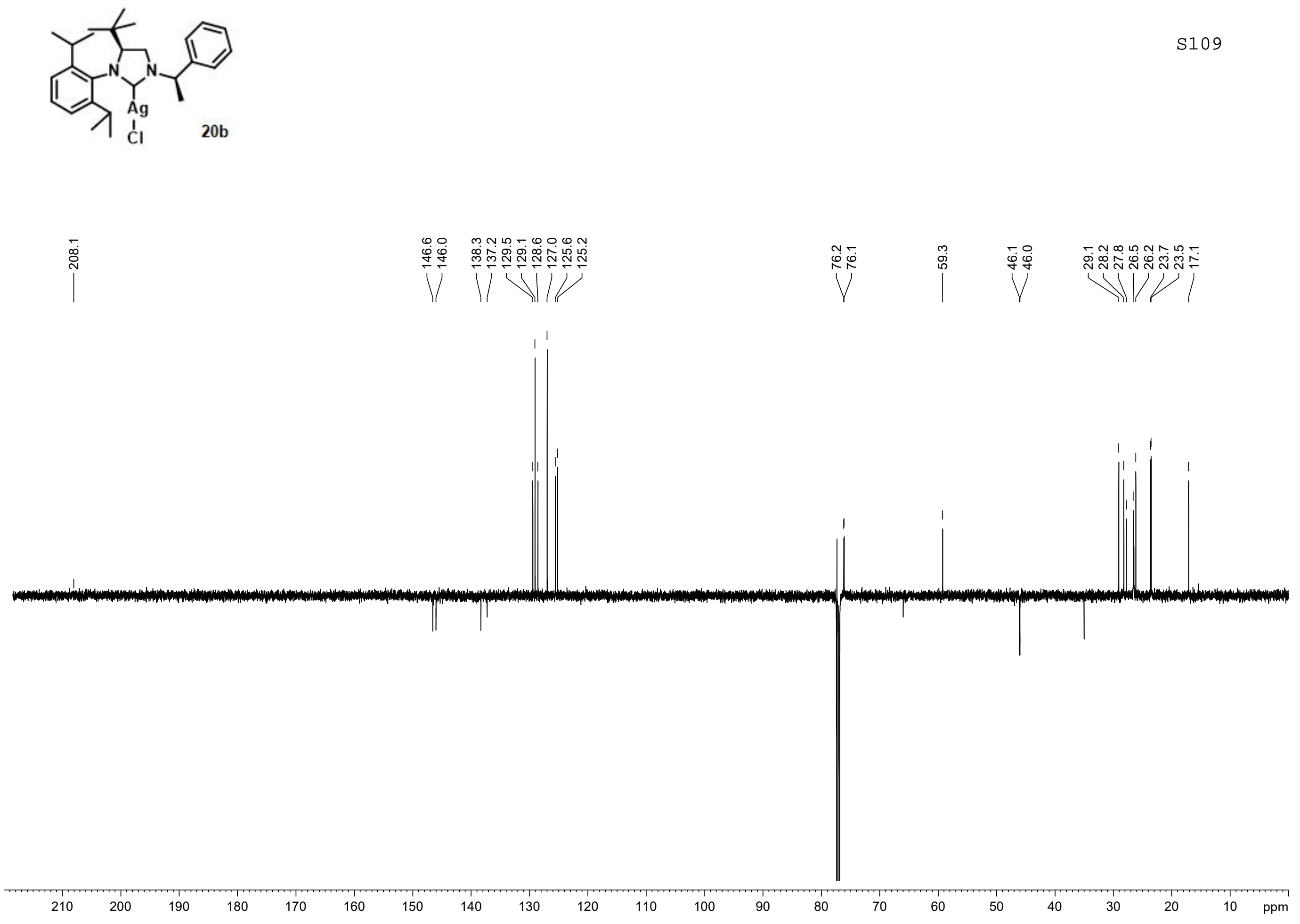

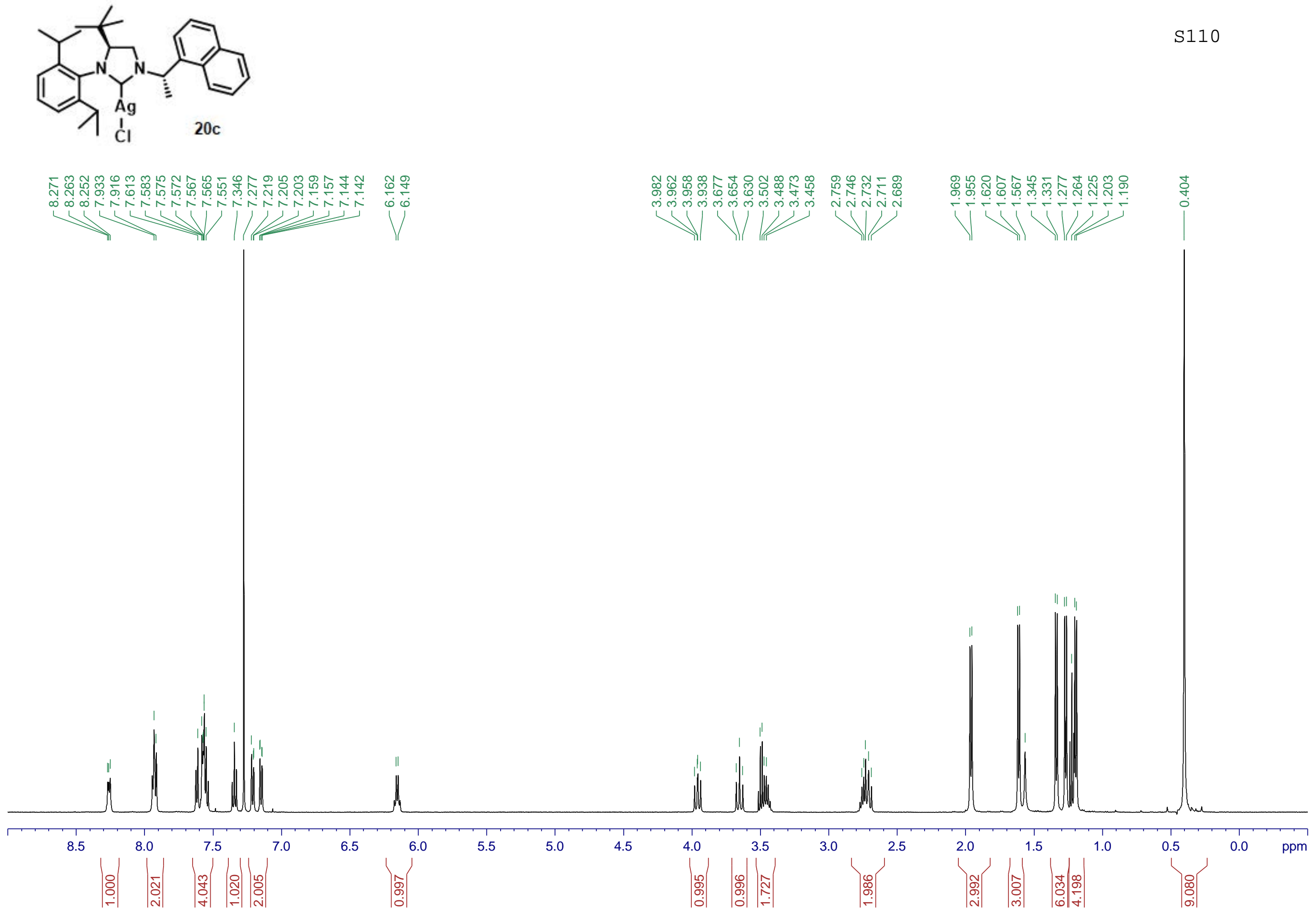



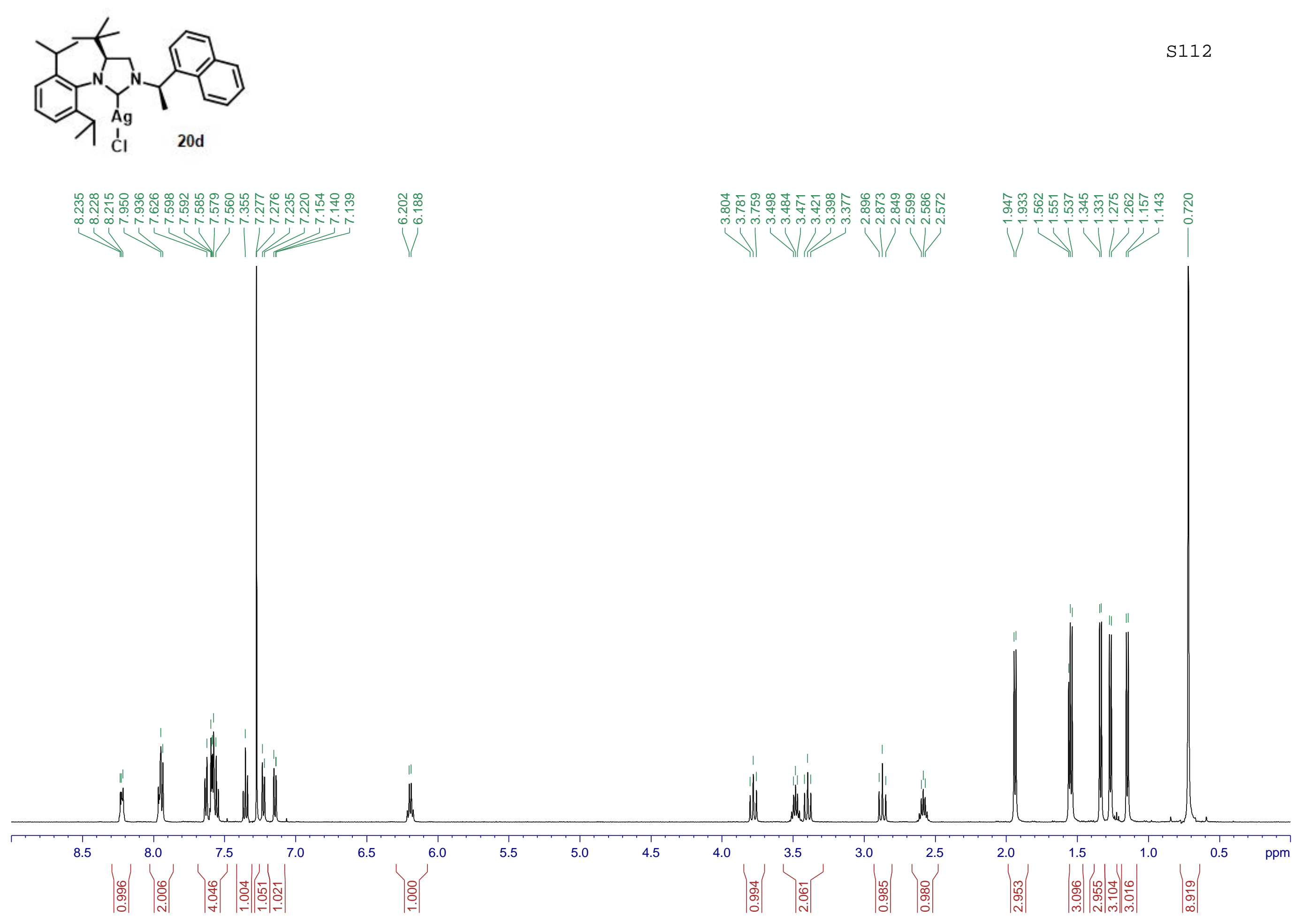

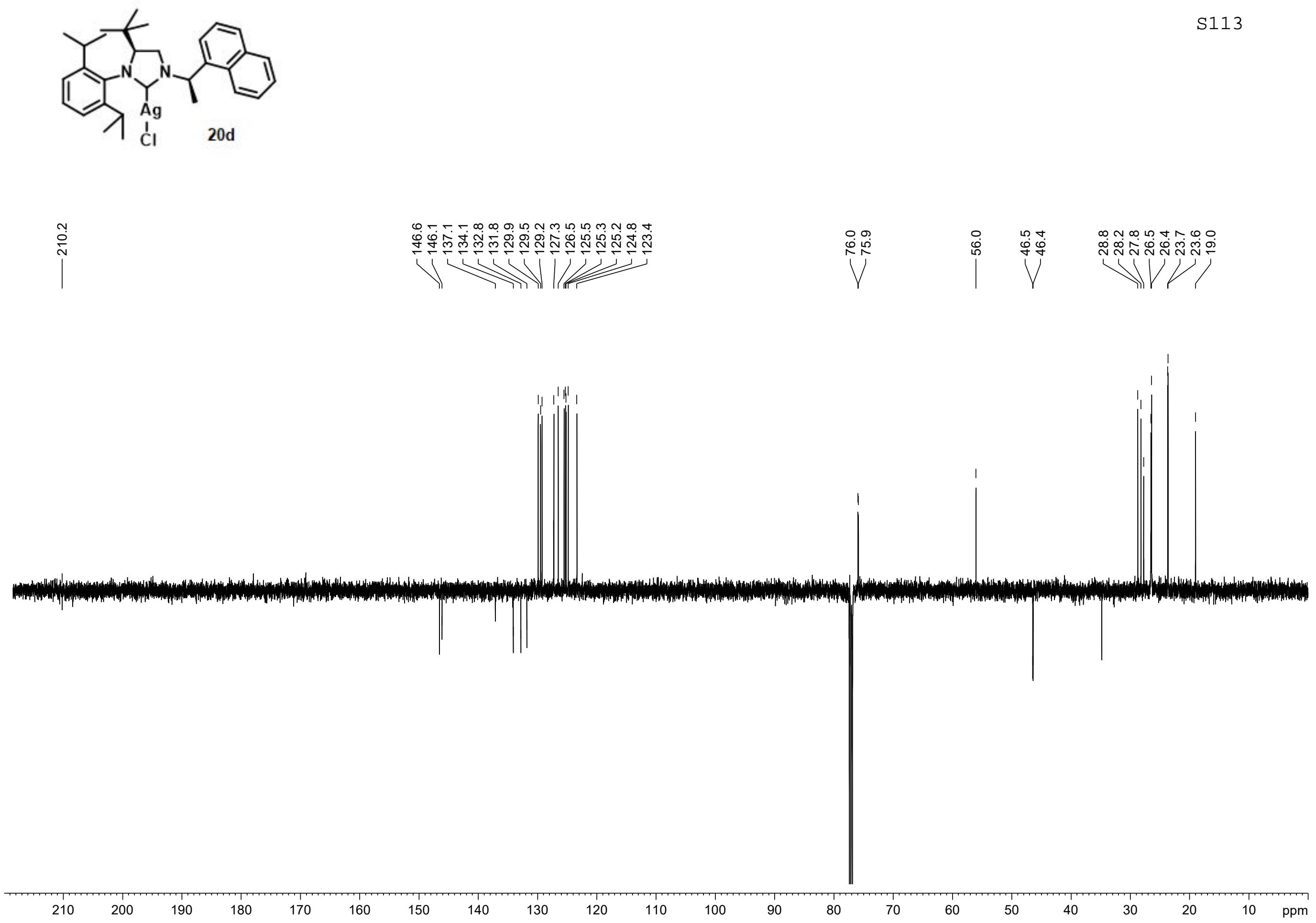


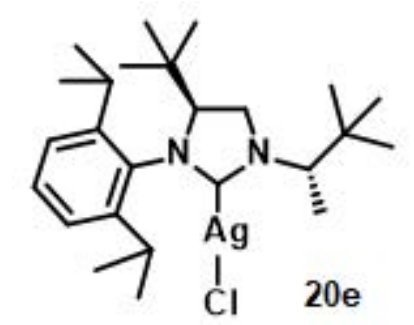

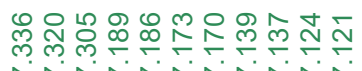

NNNNNNNN

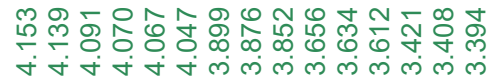

$+1$

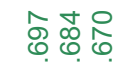

ำ



i

|

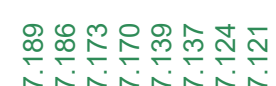

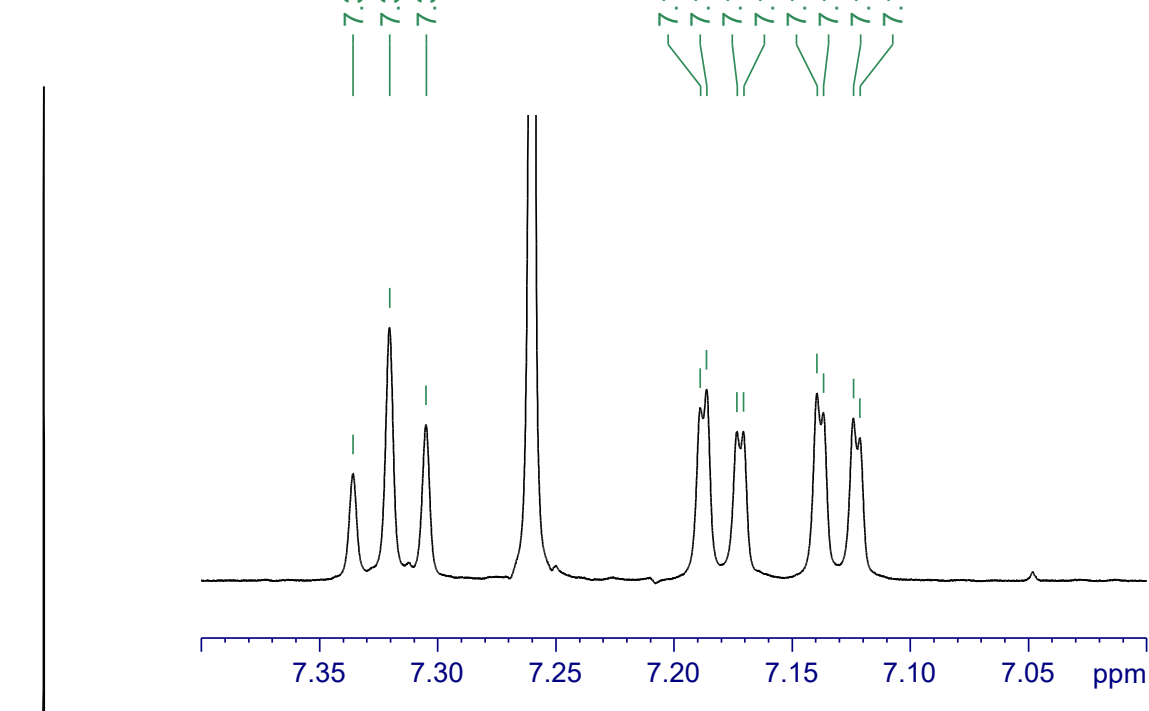

iil

What

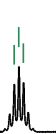
UU.

7.5 $\left|\begin{array}{l|l}\circ \\ 8 \\ 8\end{array}\right|\left|\begin{array}{l}0 \\ -\end{array}\right|$

6.5

6.0

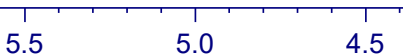

$\begin{array}{lll}4.0 & 3.5 & 3.0\end{array}$

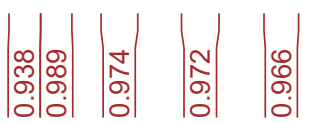

$\left|\begin{array}{c}m \\ m \\ 0 \\ 0 \\ 0\end{array}\right|$

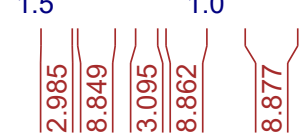




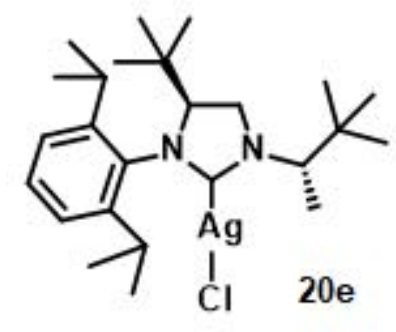

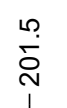

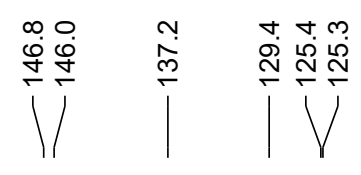

$\left.\right|^{m}$

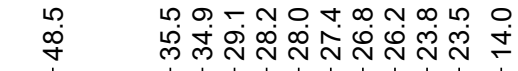

1
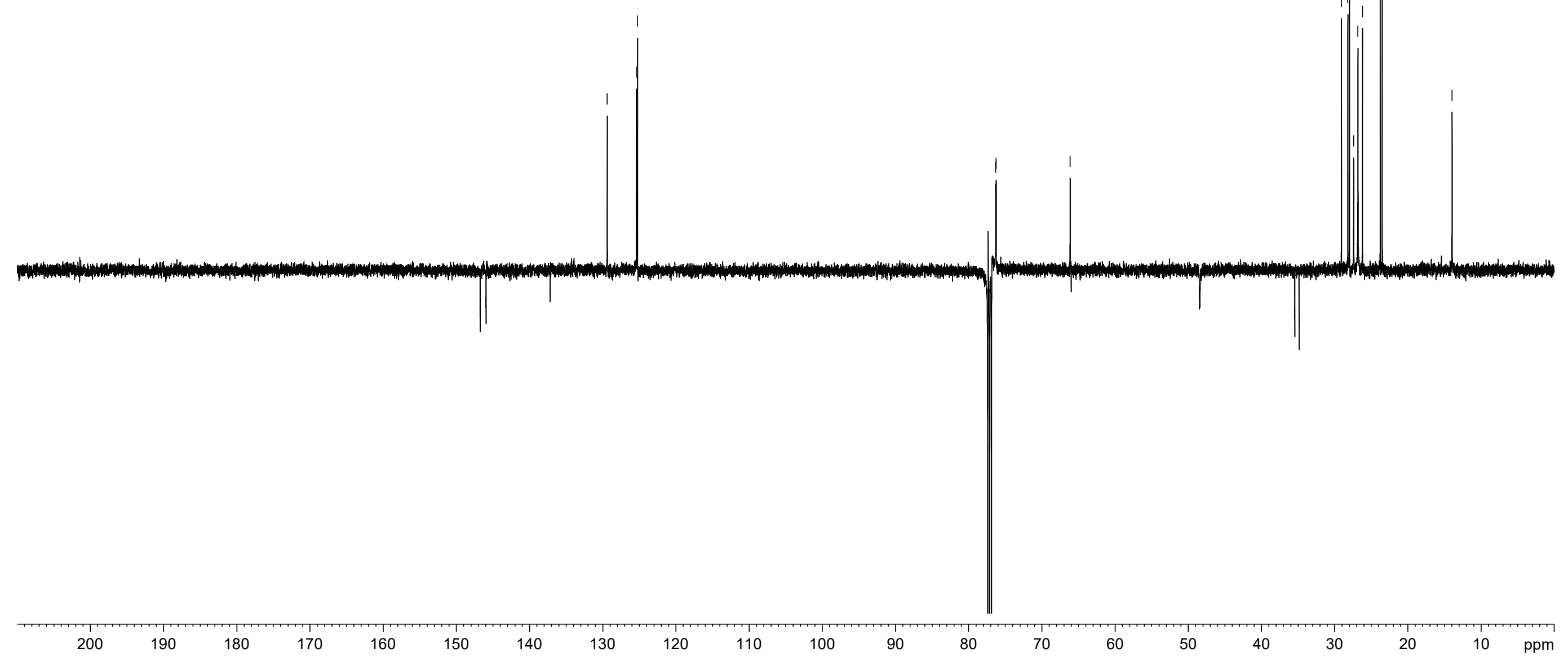


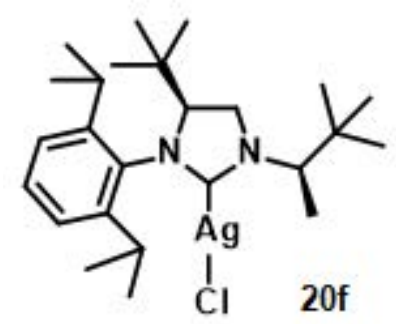

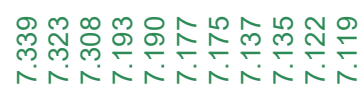

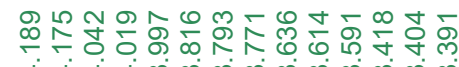

용워

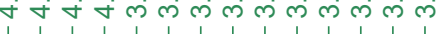

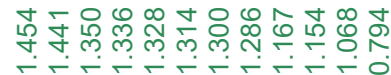
$\longrightarrow 1$
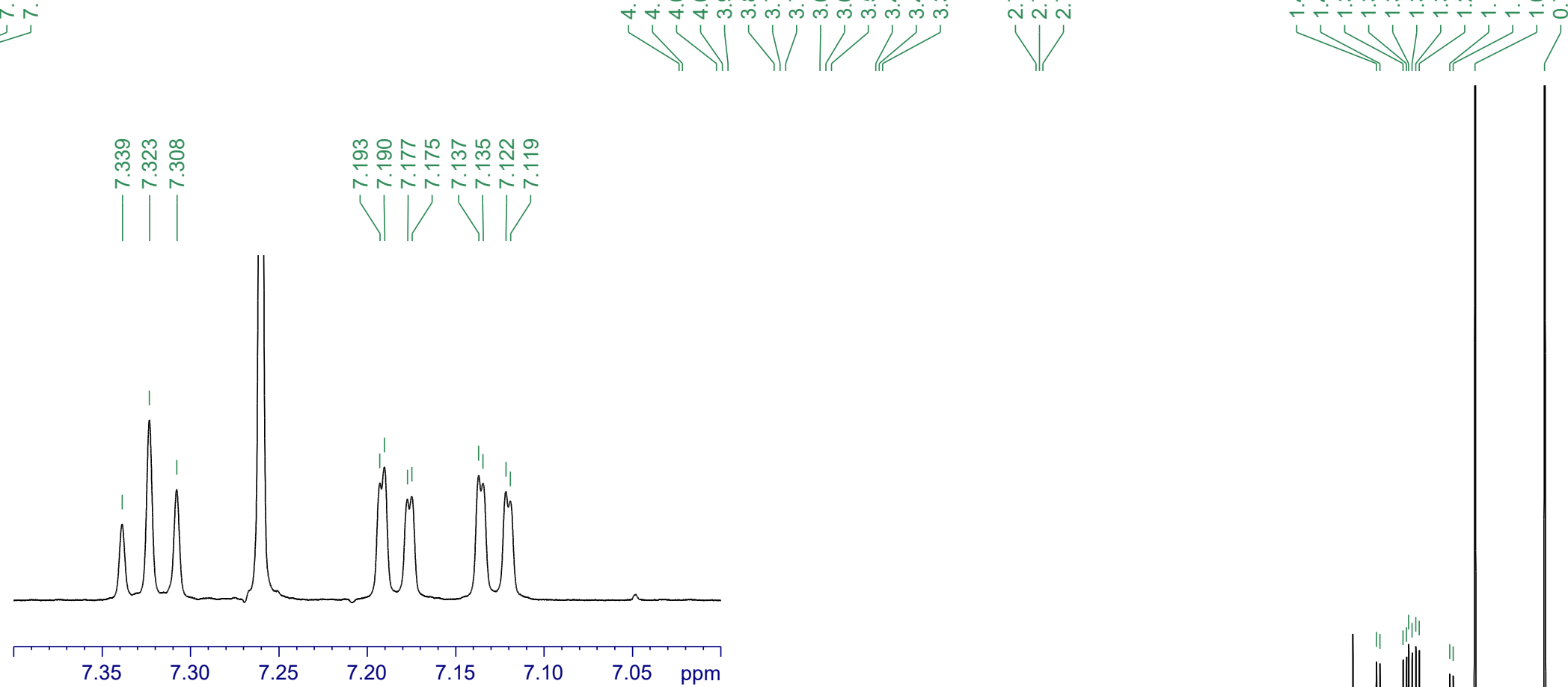

HW
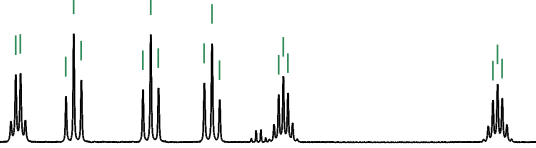

7.5 $\left|\begin{array}{l|c}0 \\ 0 \\ 8 \\ \hdashline\end{array}\right|$ 

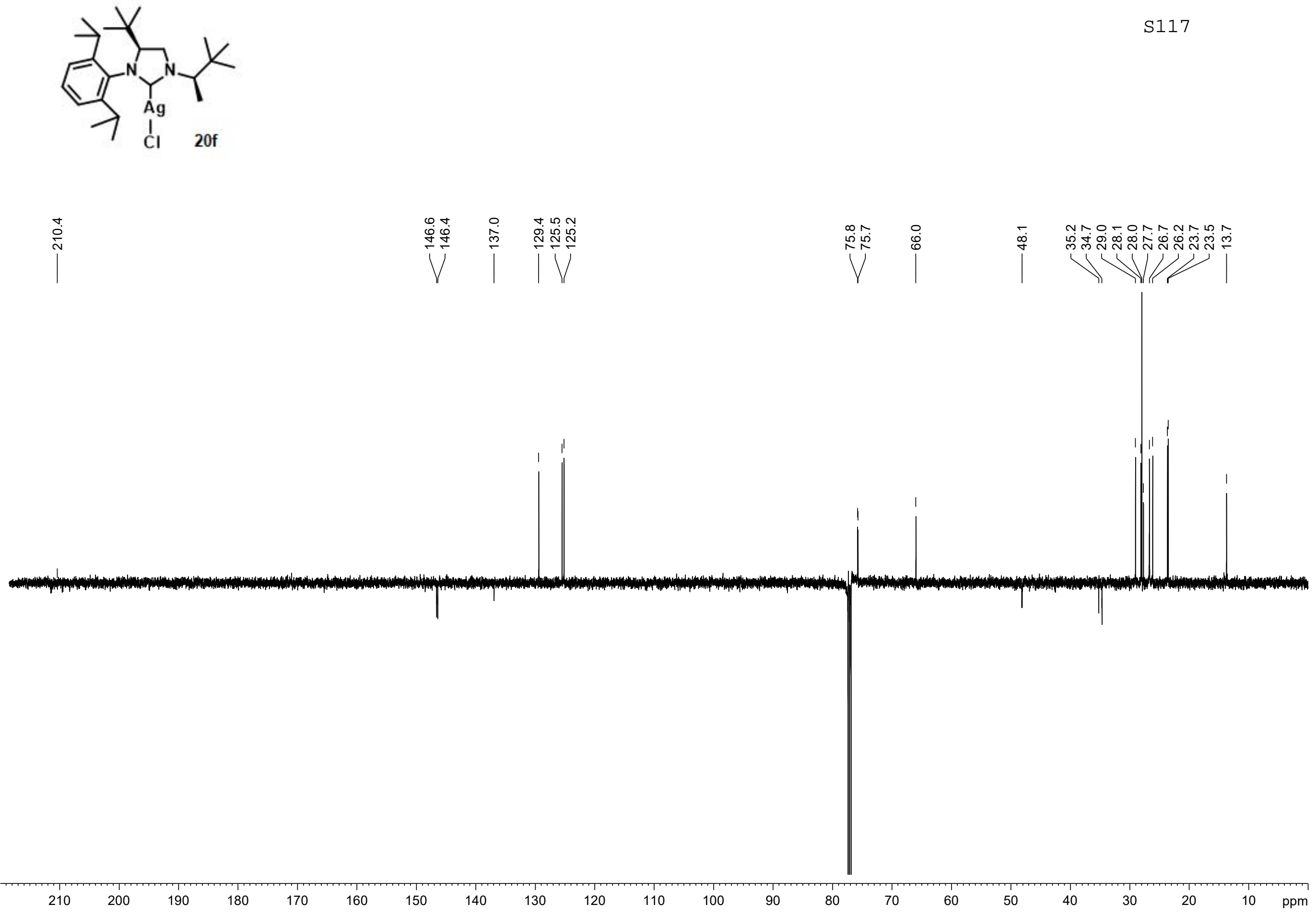

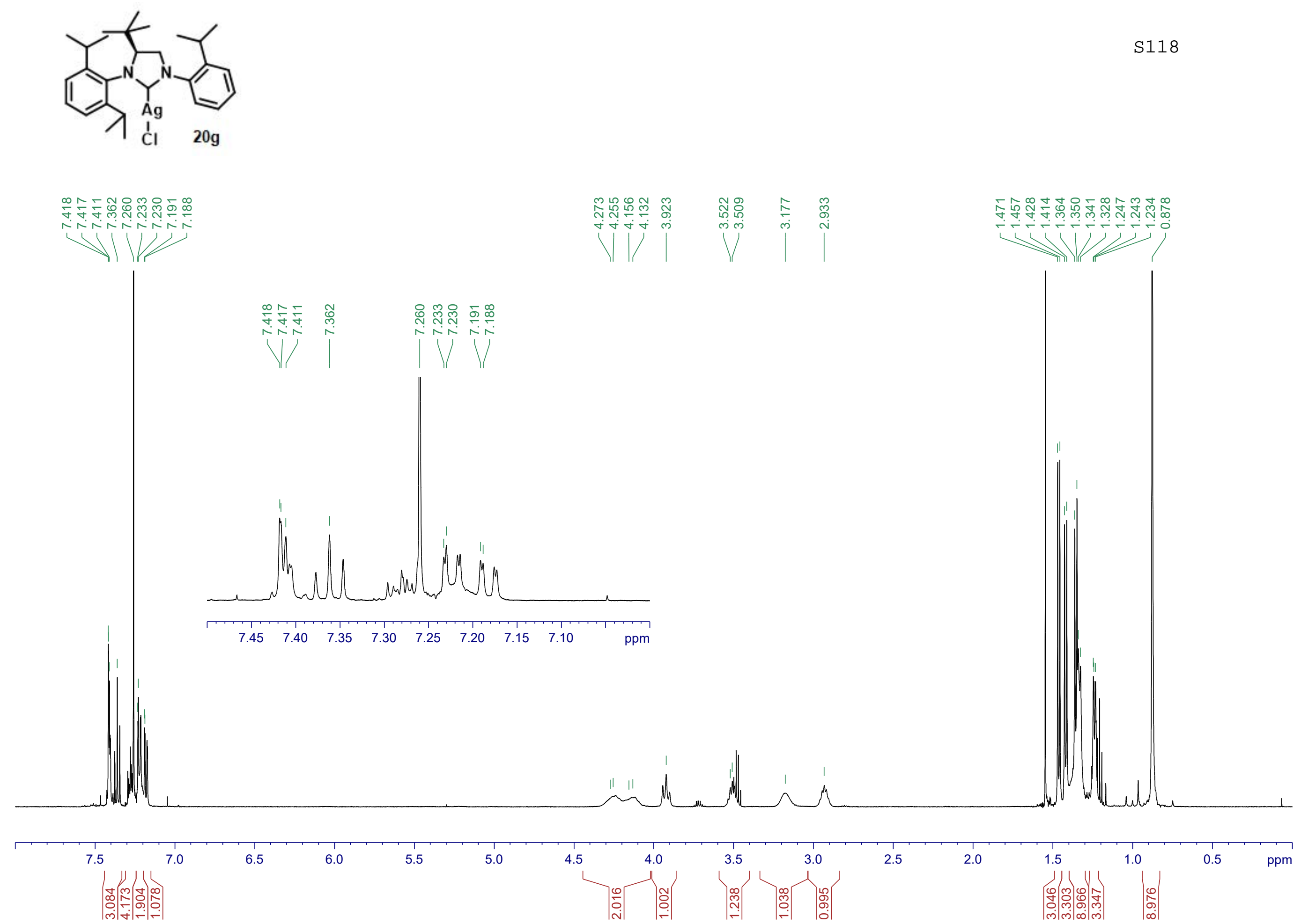


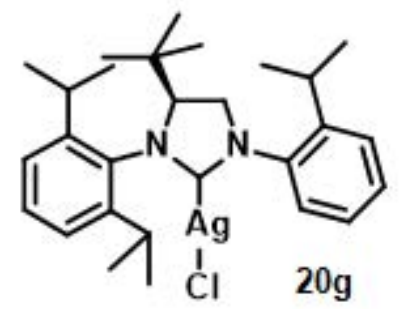

$\stackrel{\circ}{\stackrel{\infty}{\circ}}$

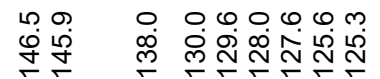

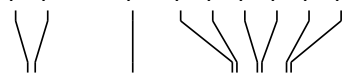

m

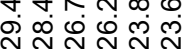

111

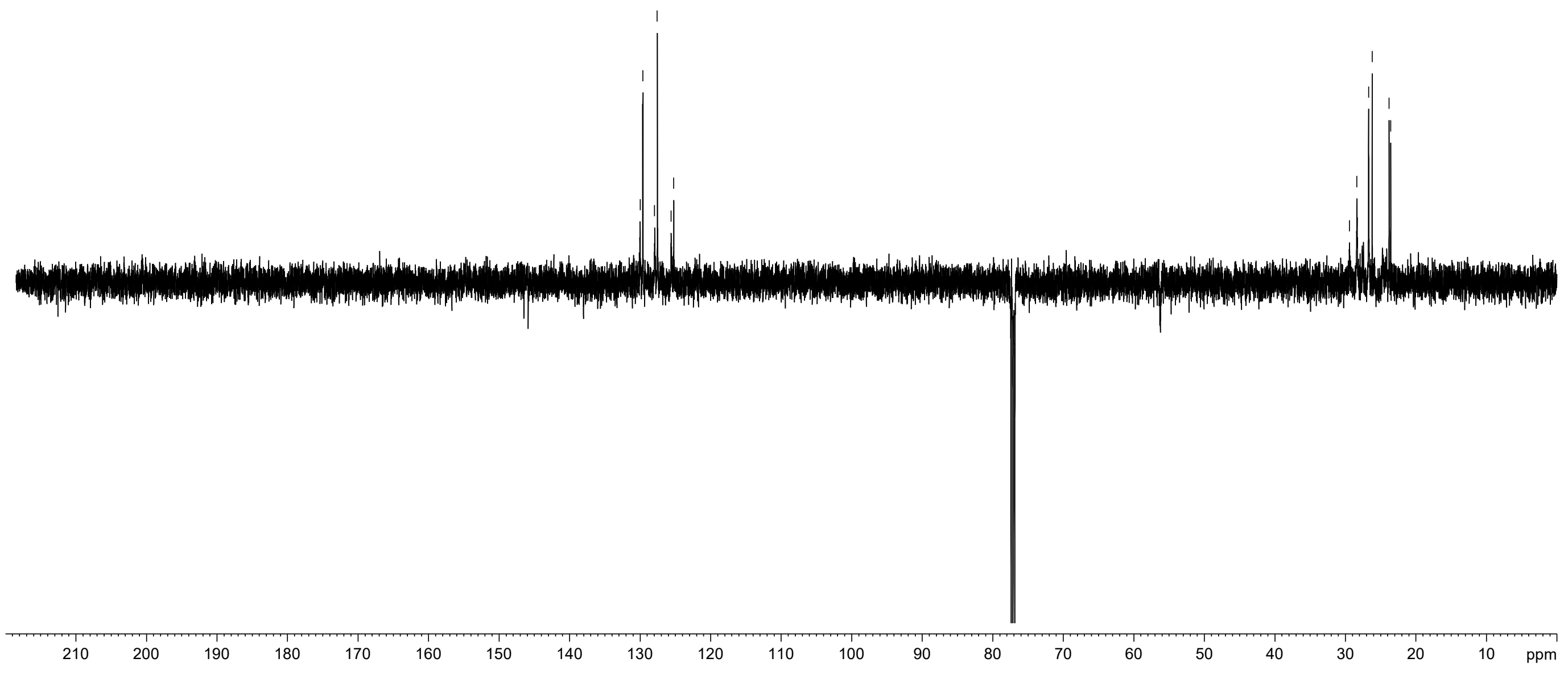



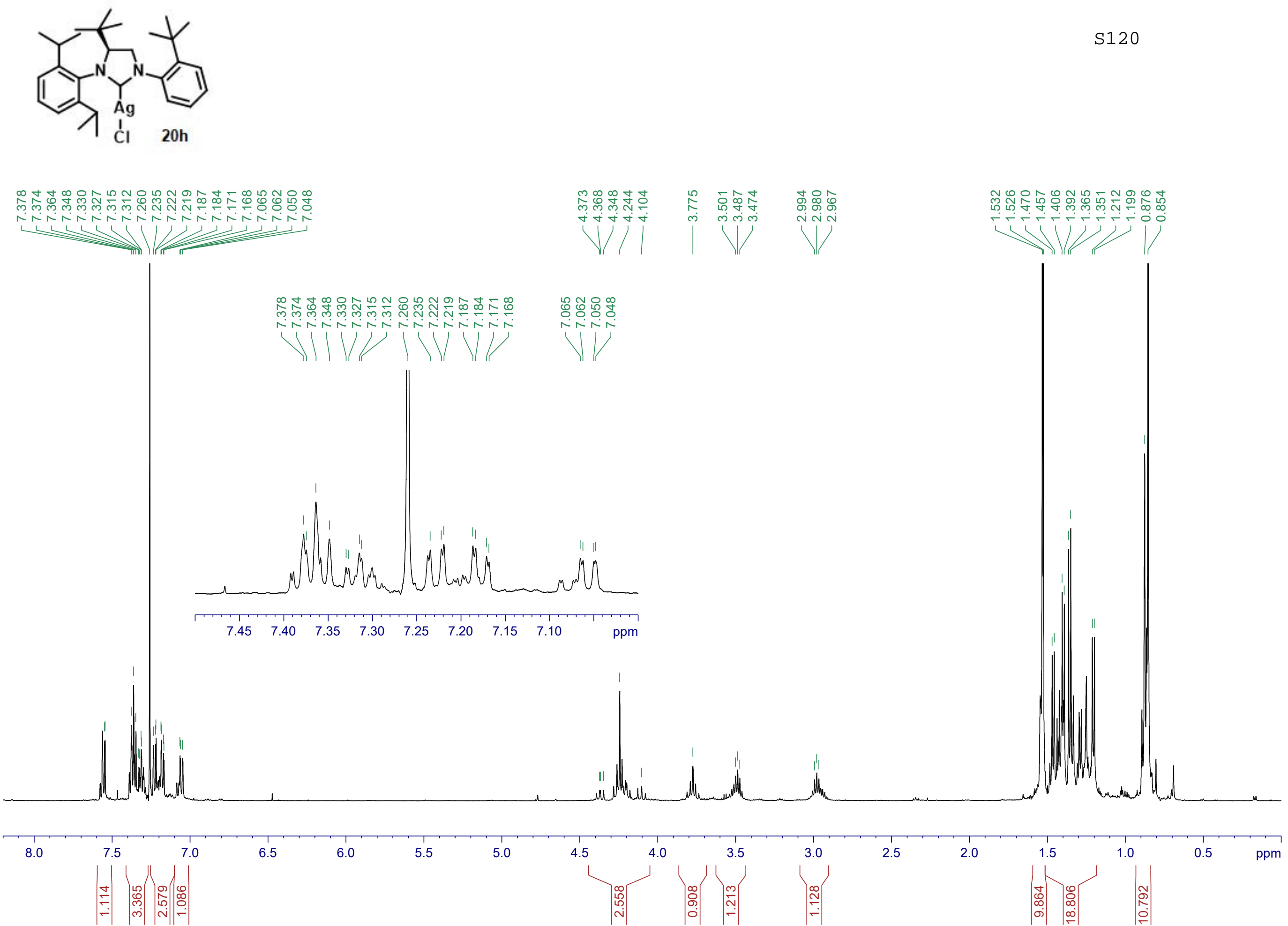

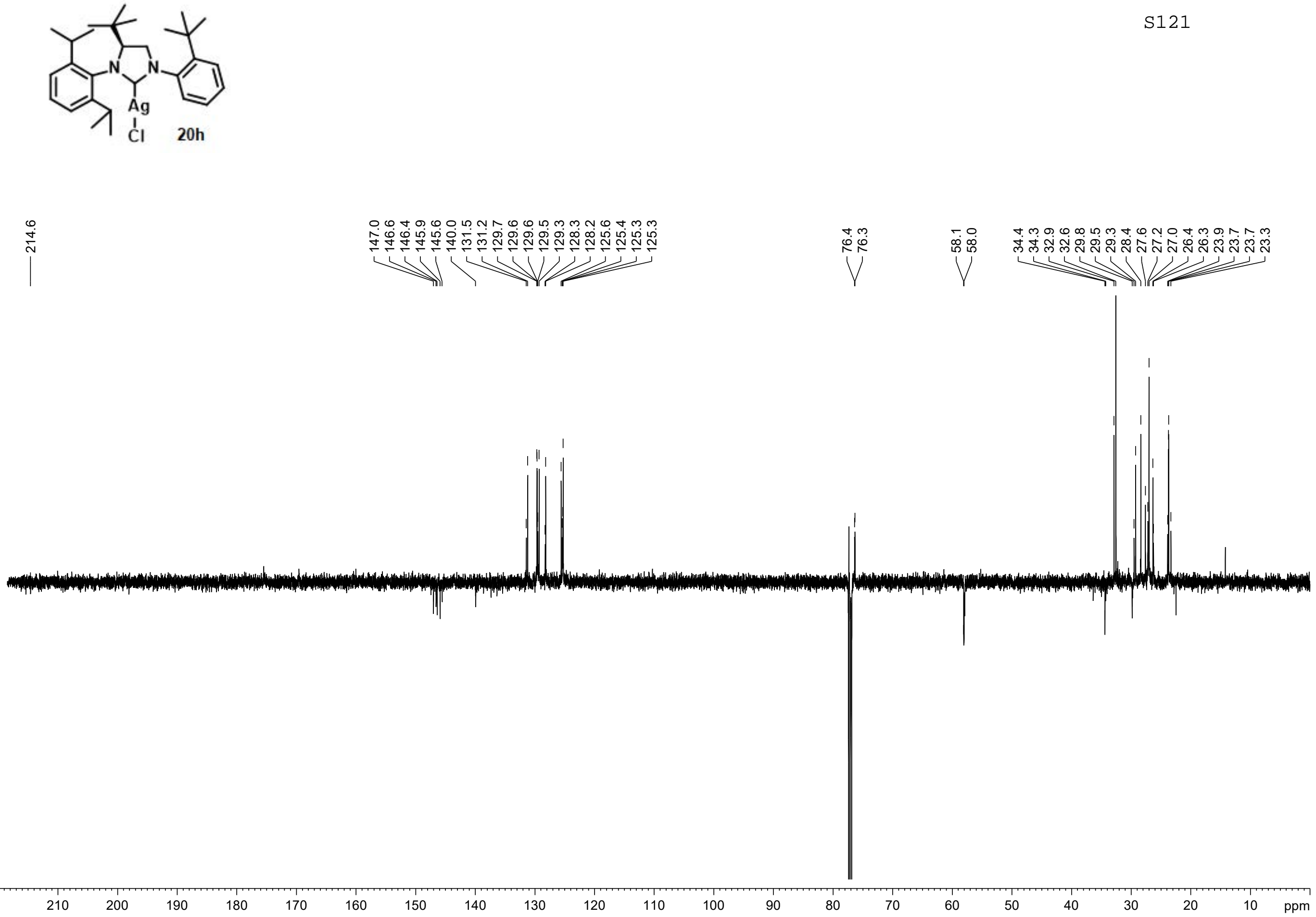


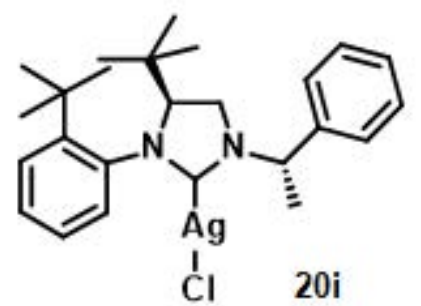

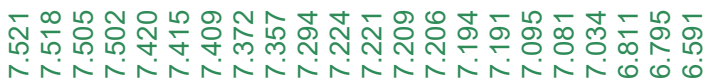

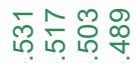




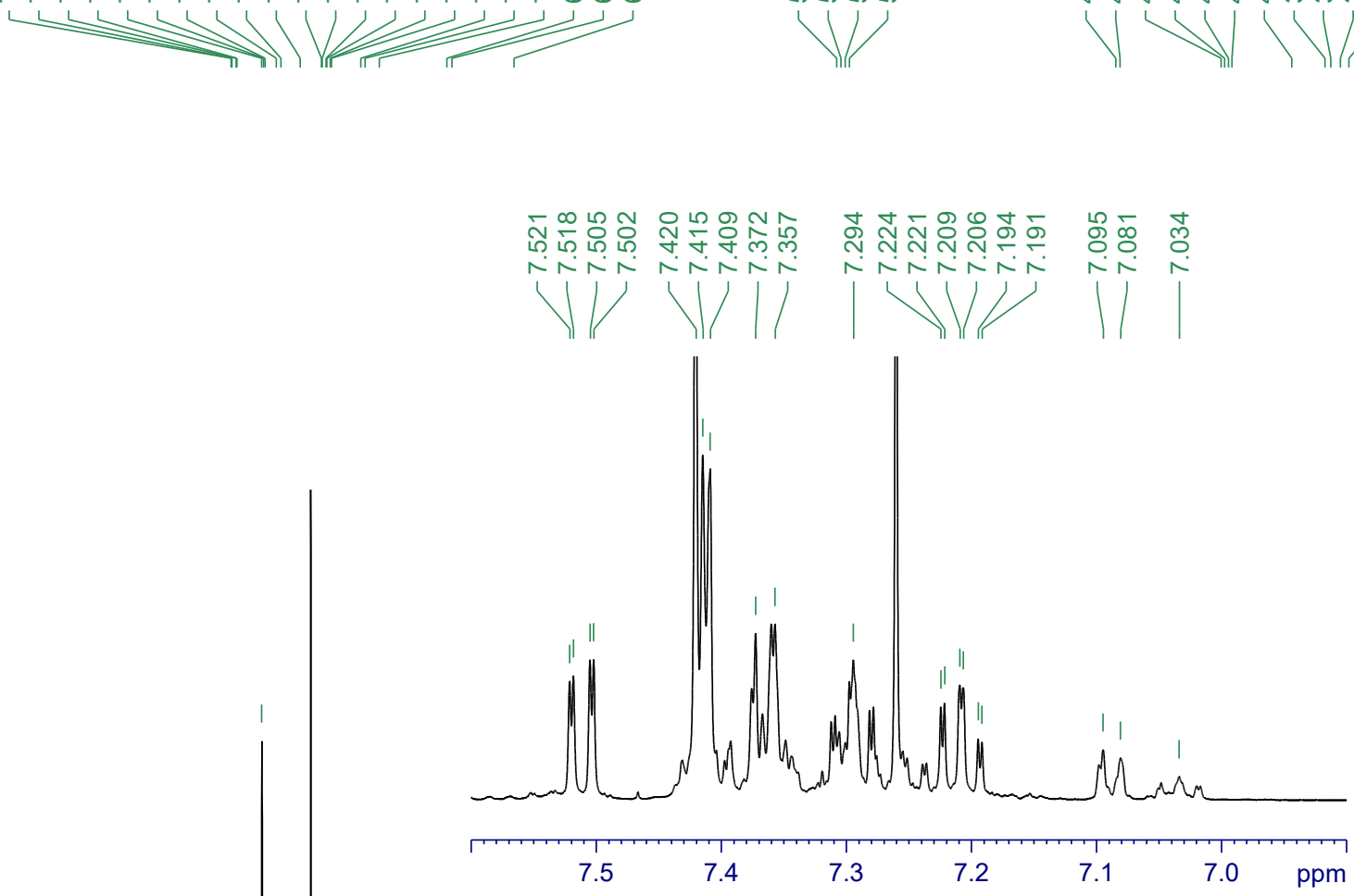

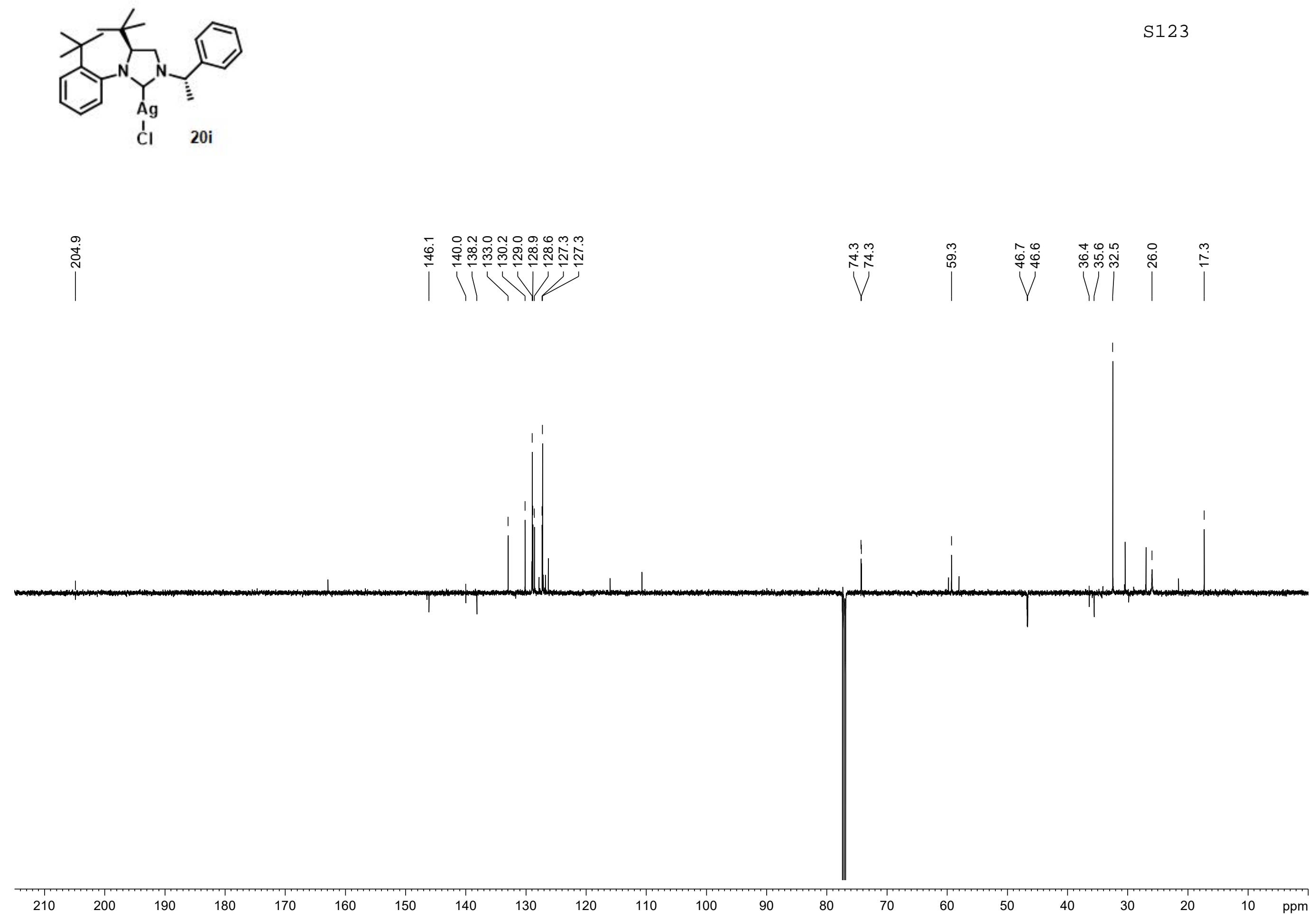




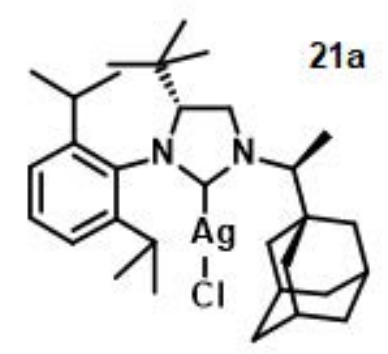

$\stackrel{9}{\circ}$

V)

$\mid$

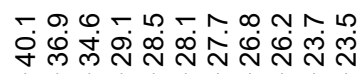
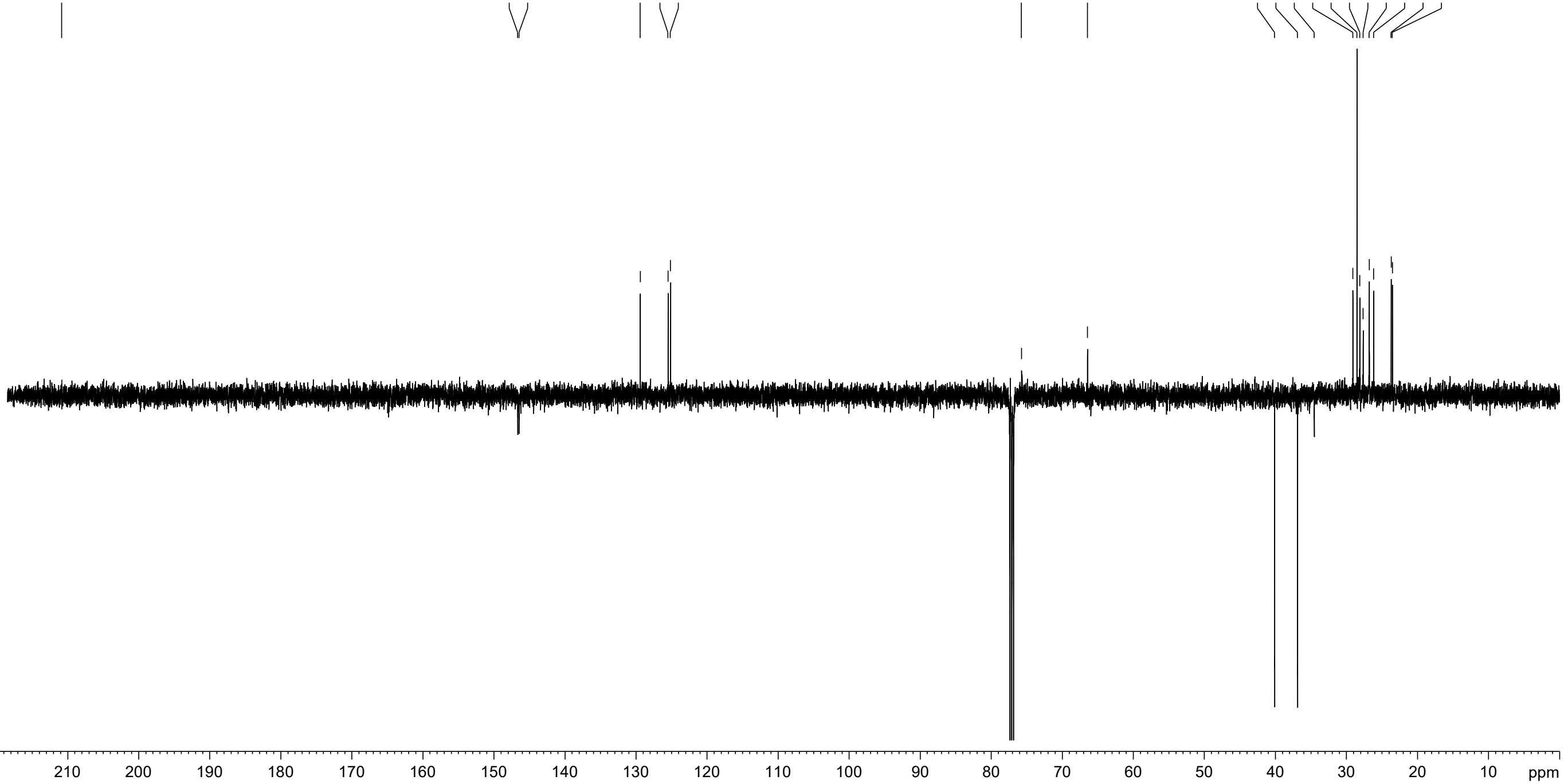

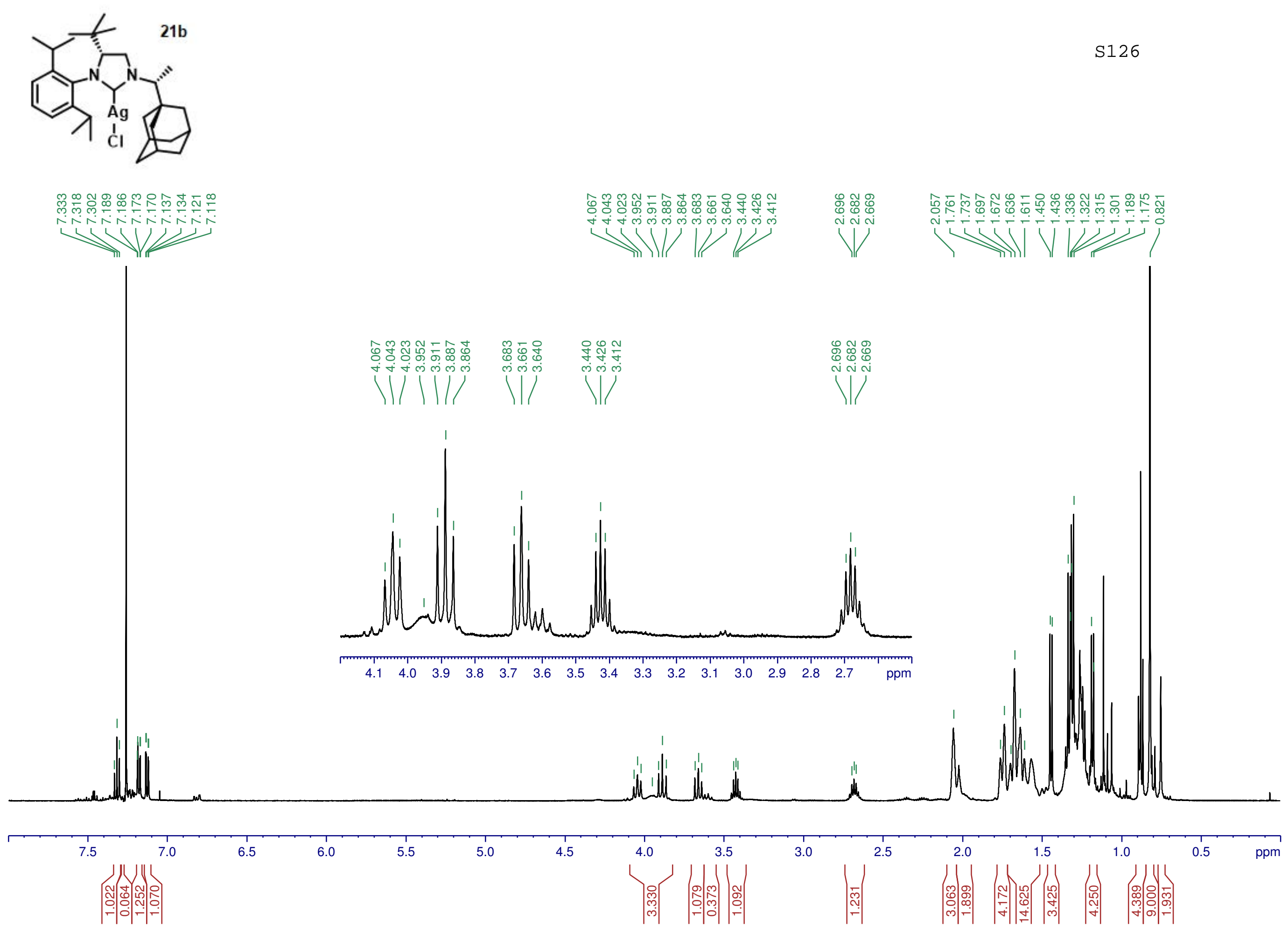


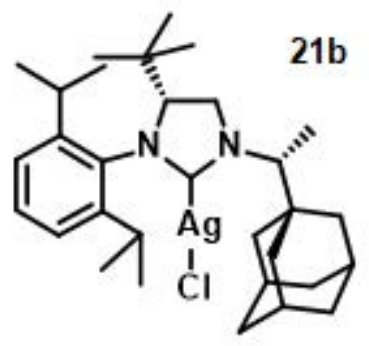

ํㅜㅇ

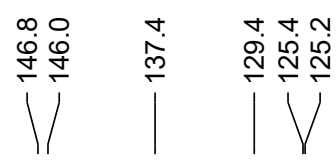

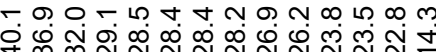
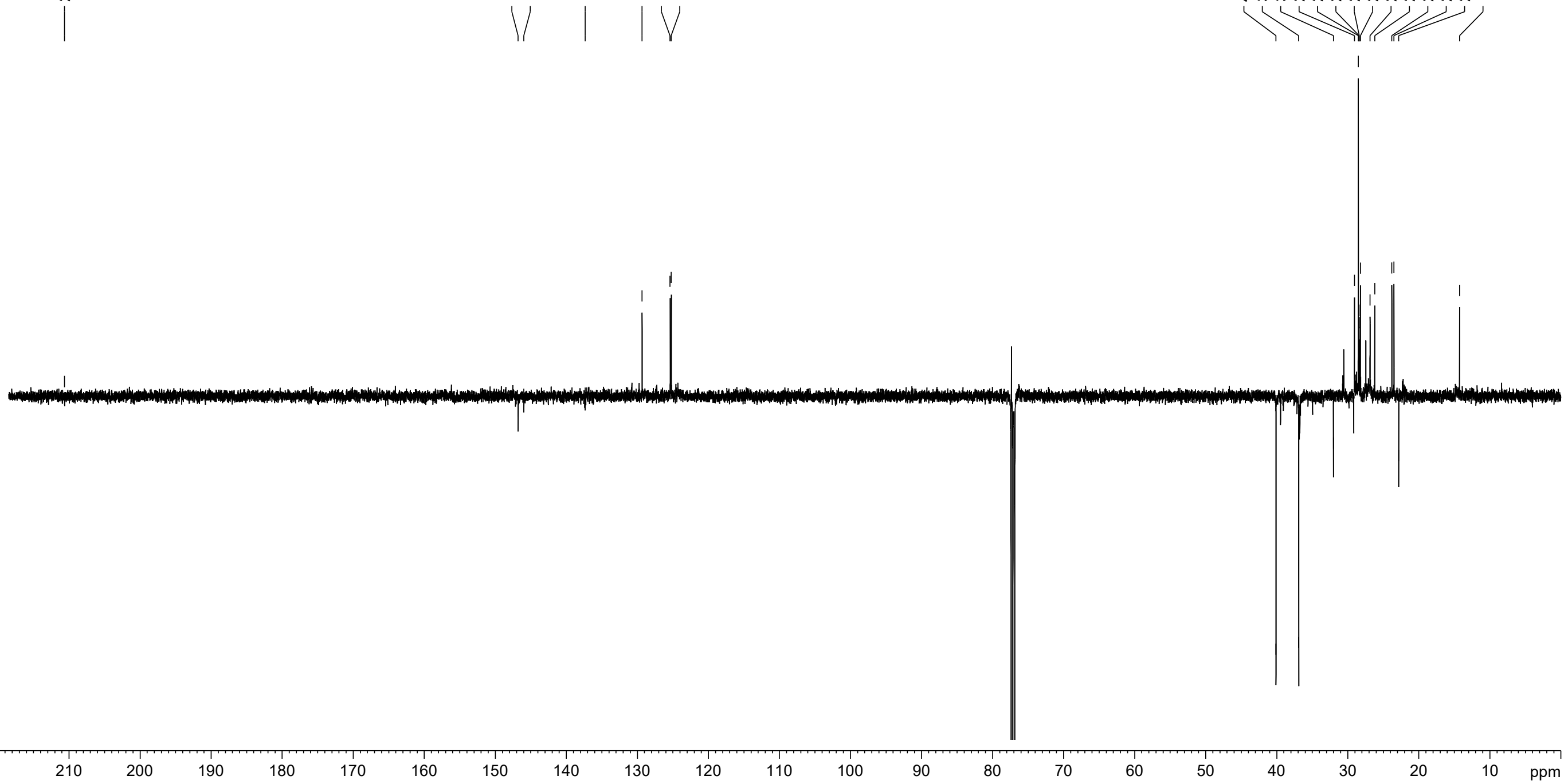

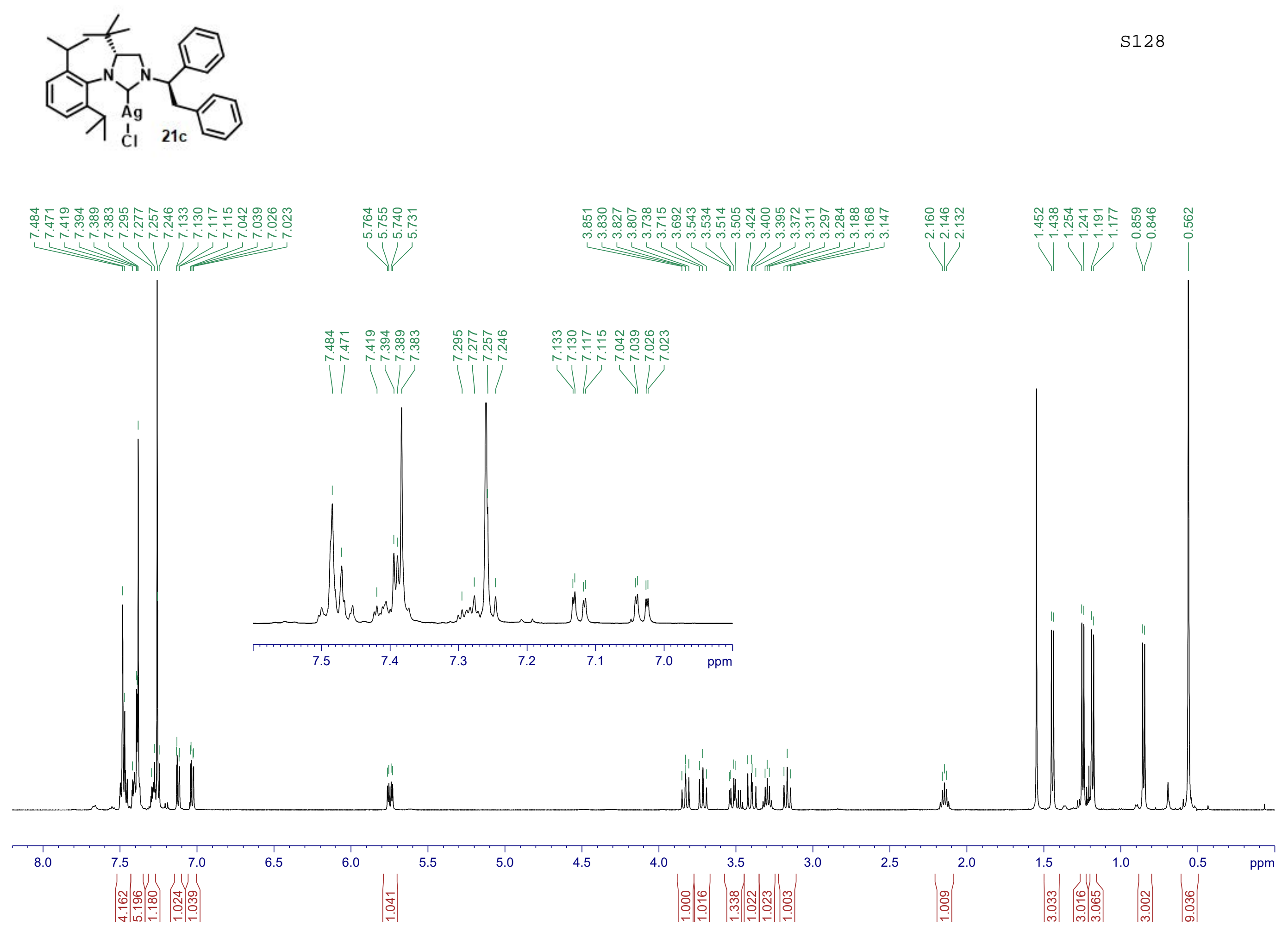

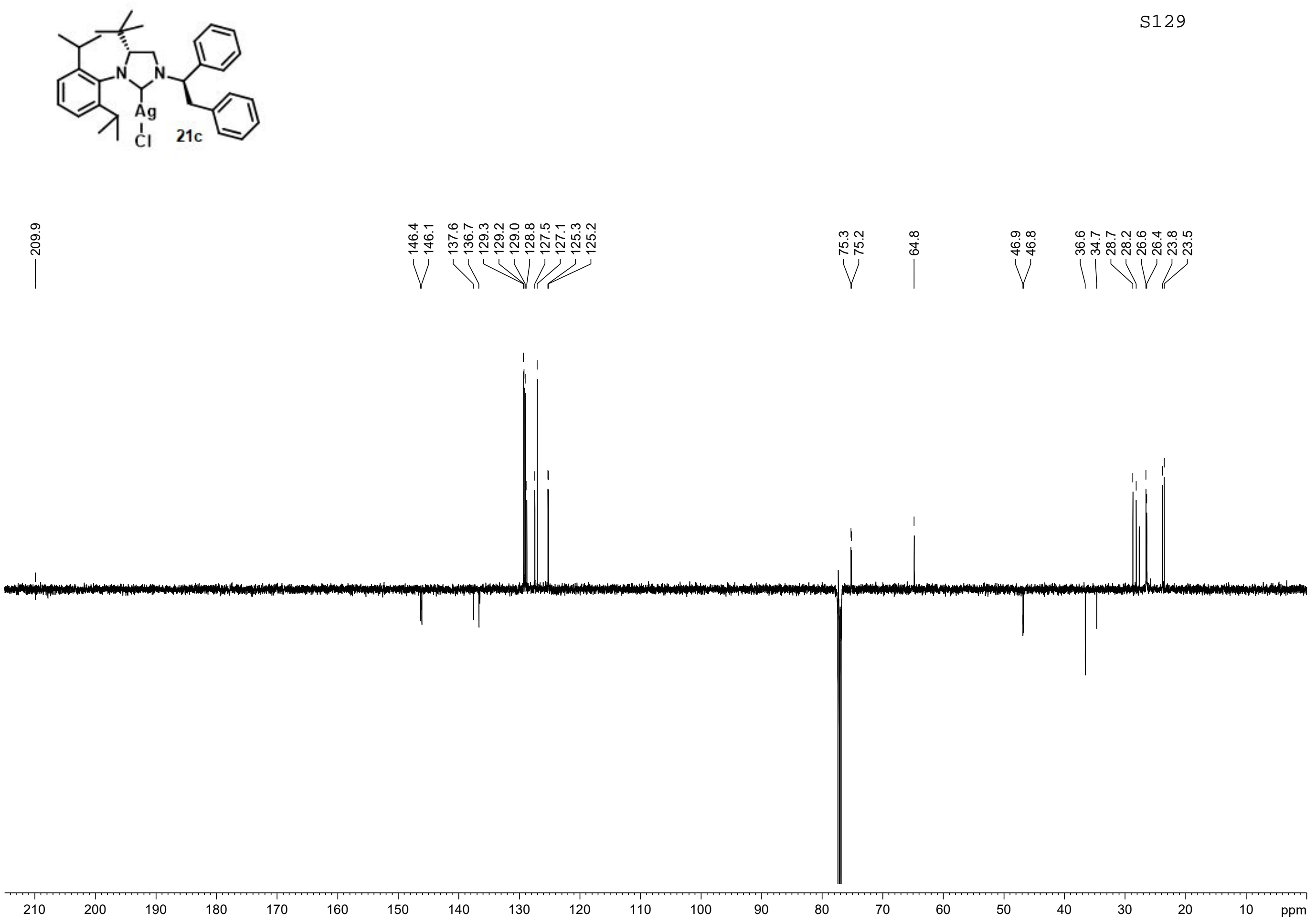

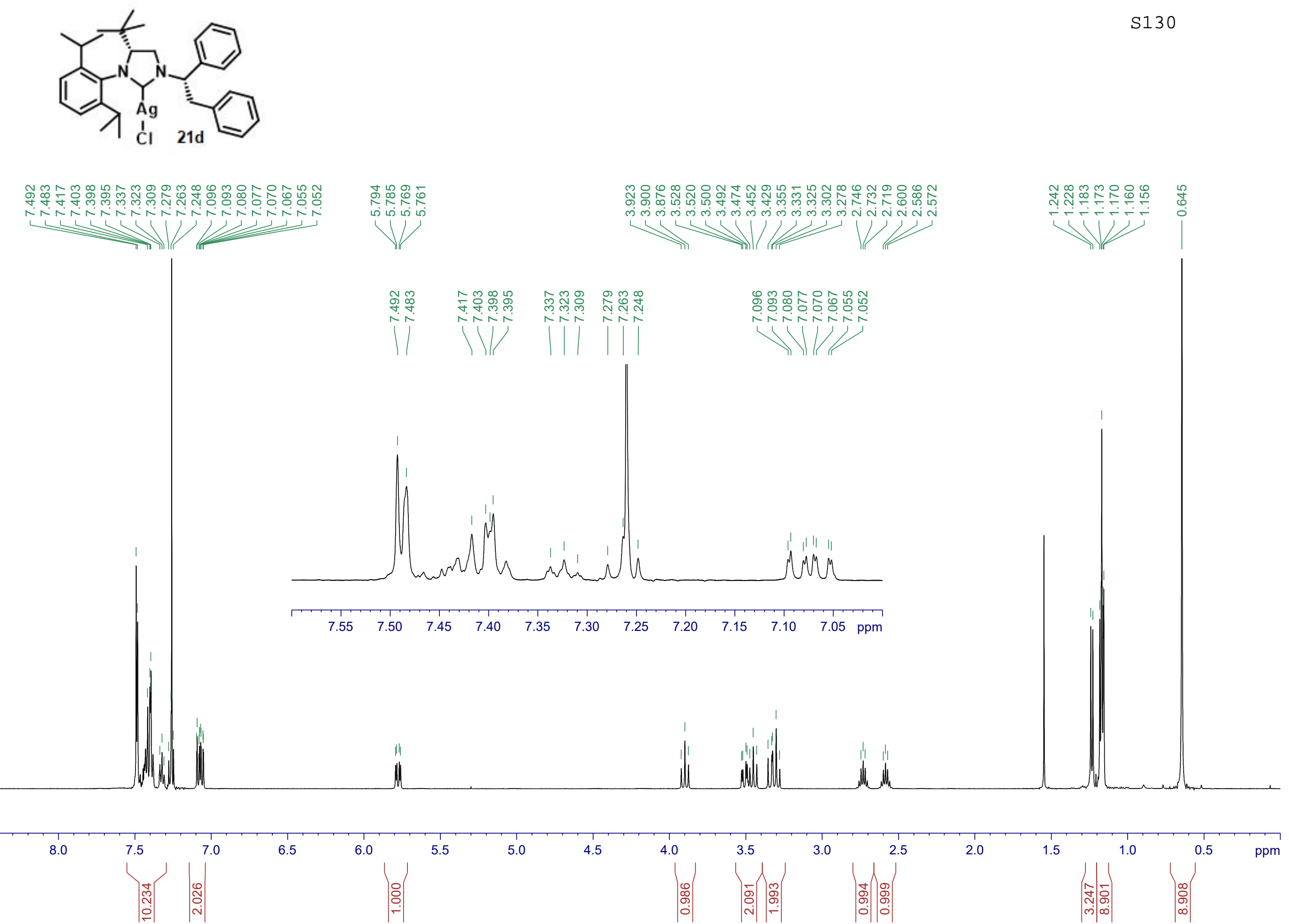

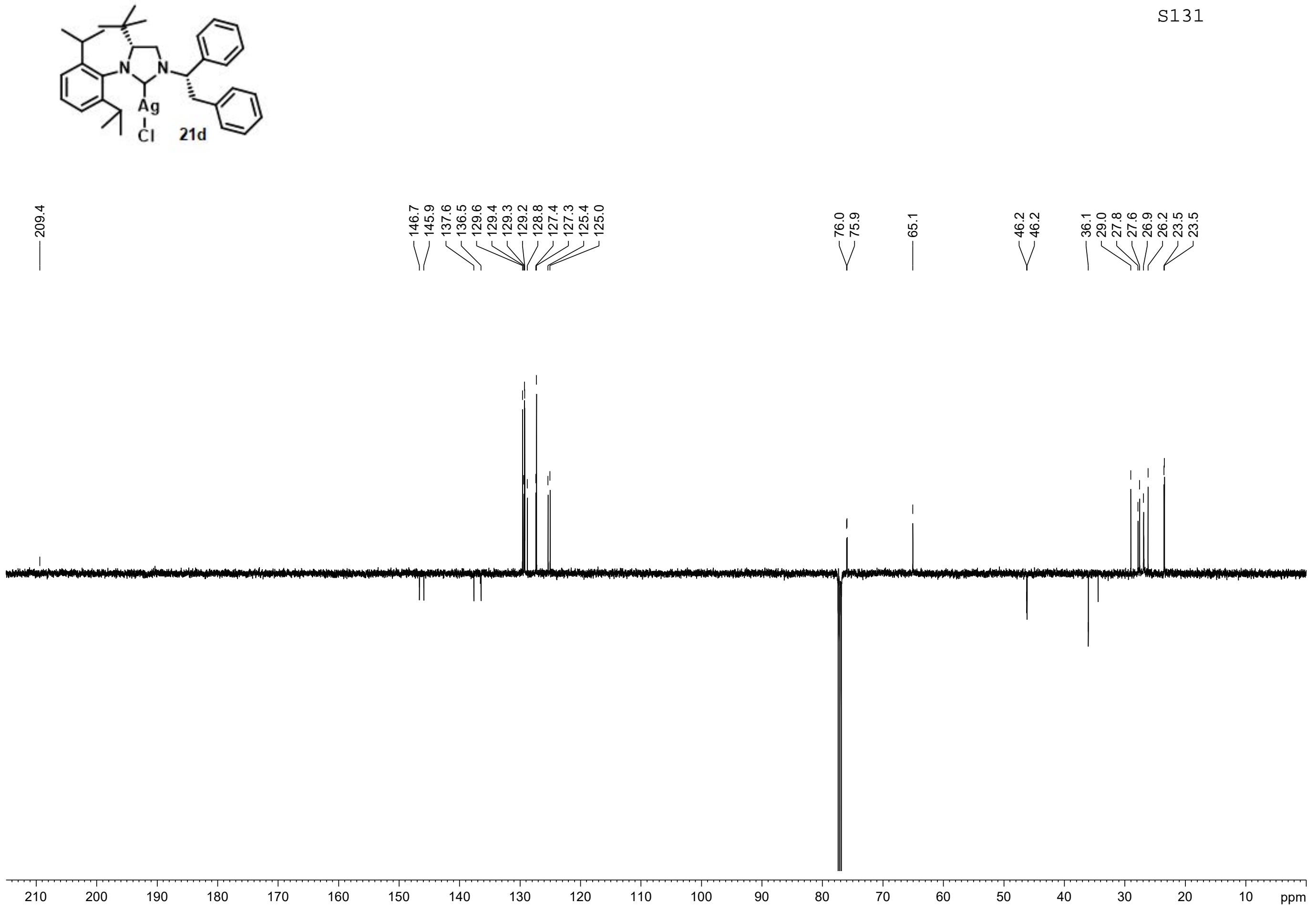

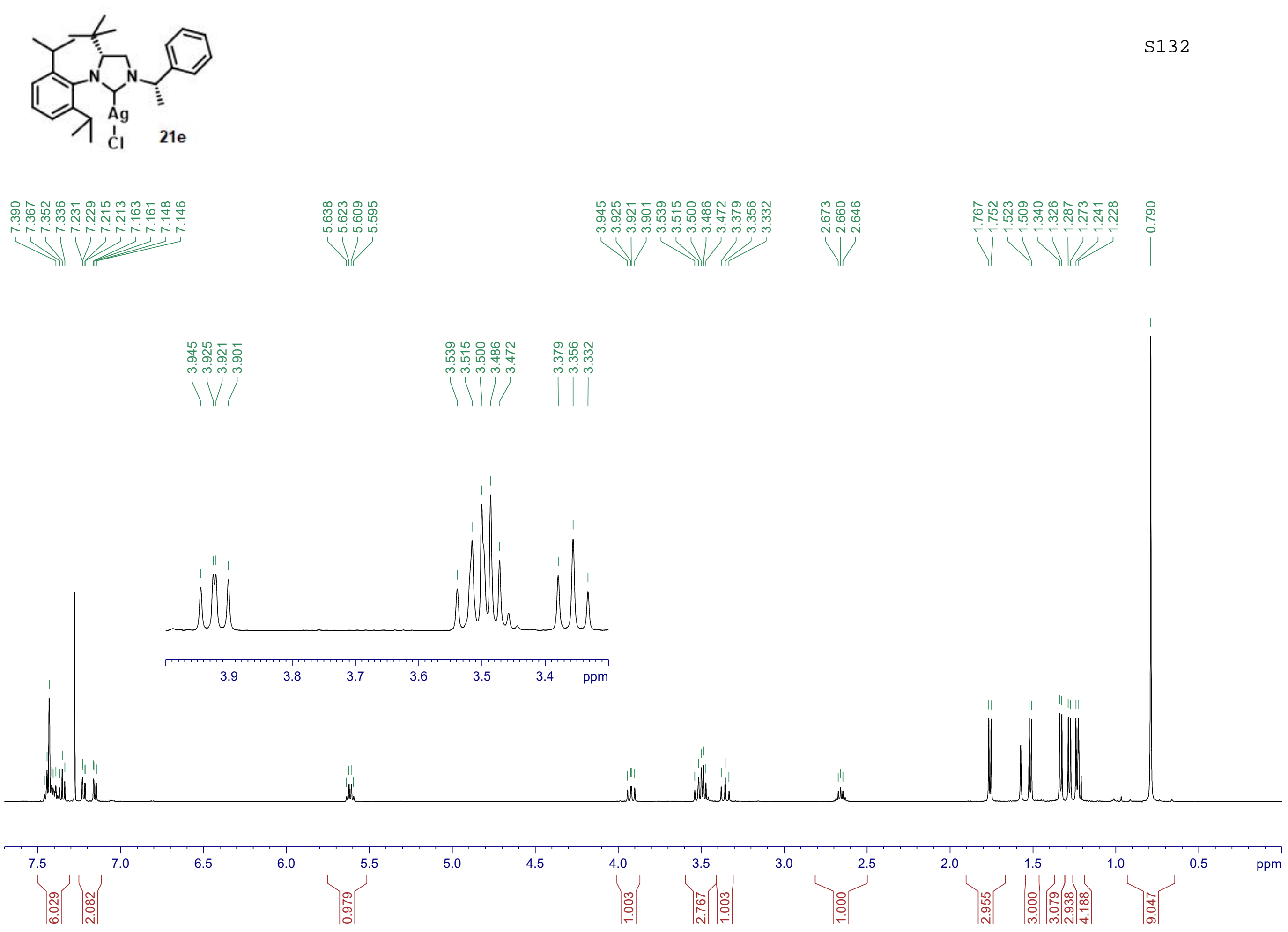

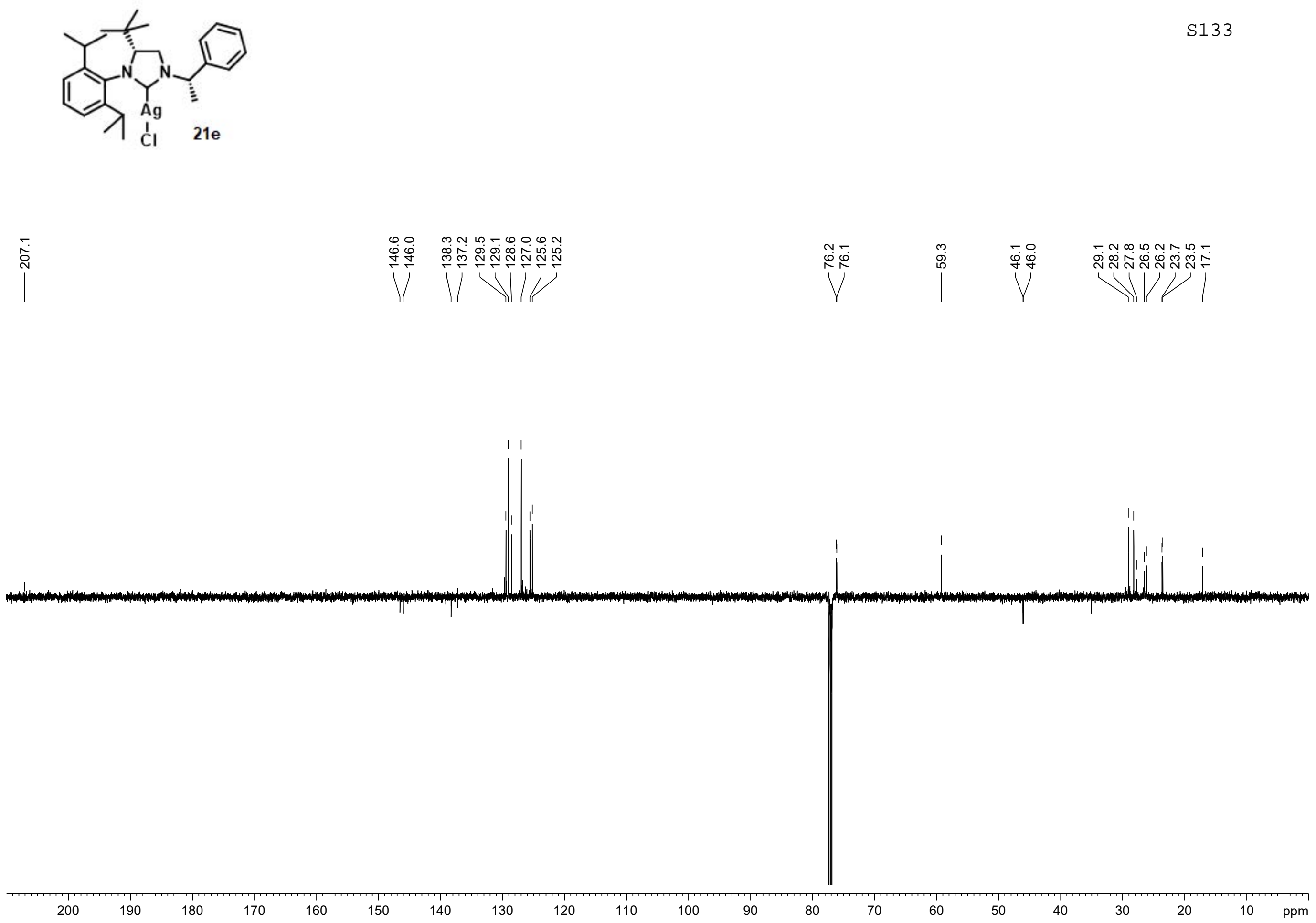

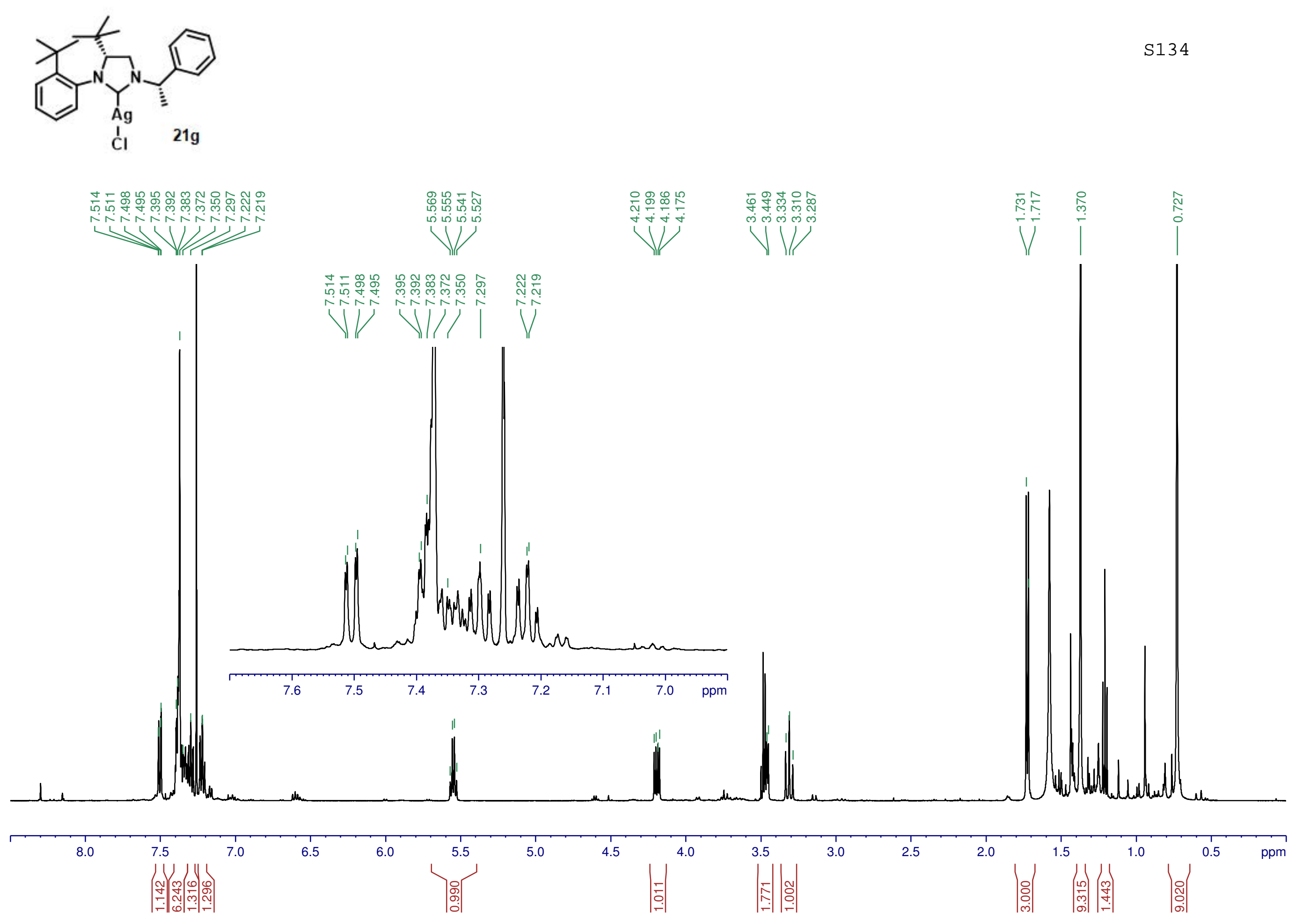

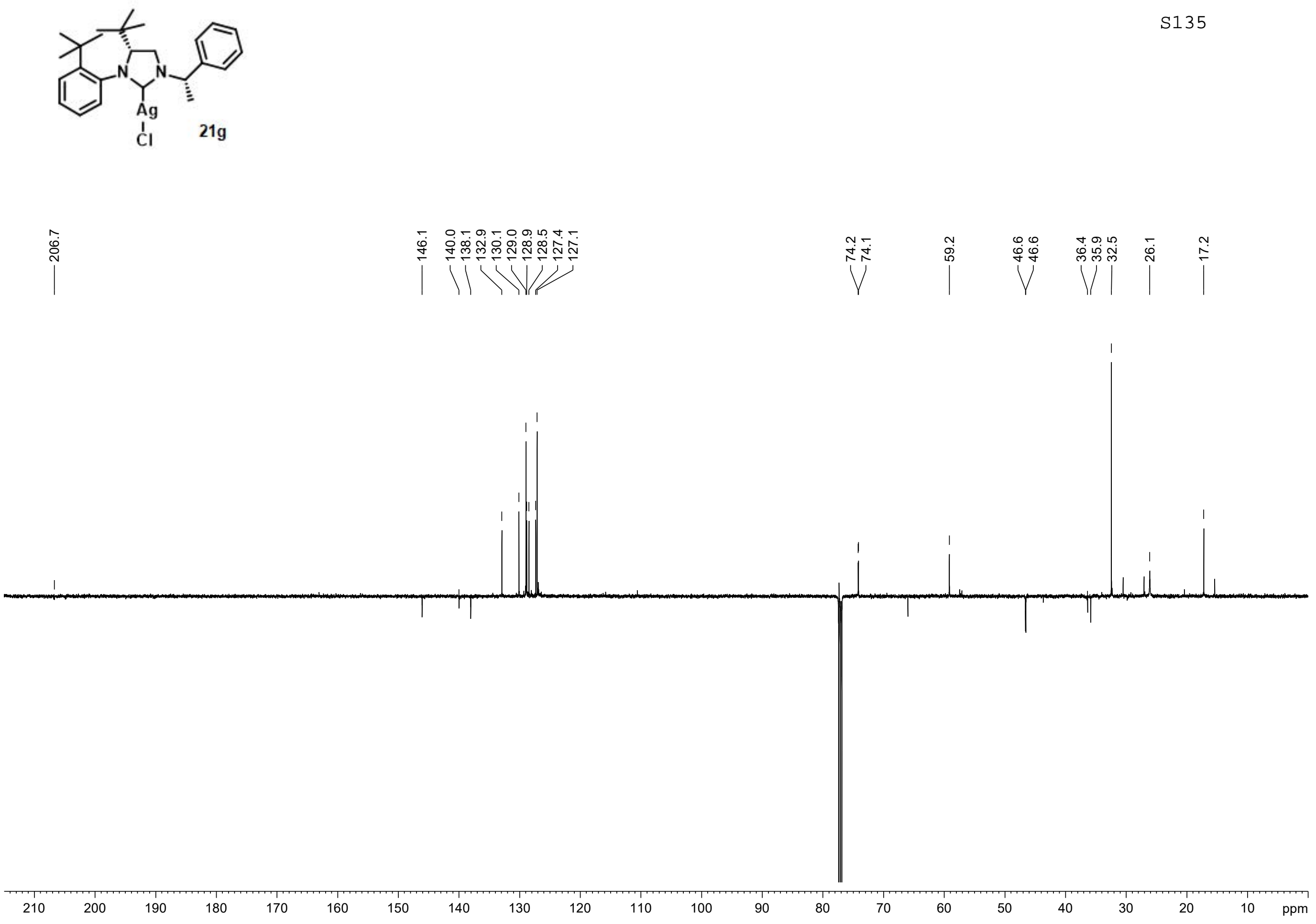


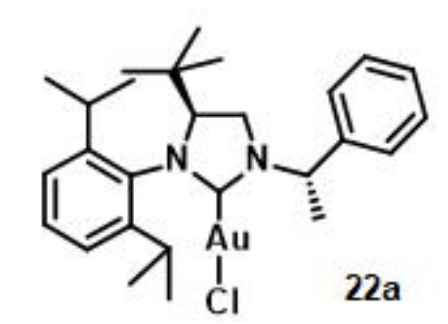

\section{$\underbrace{2}$}

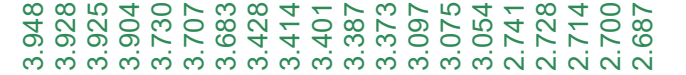

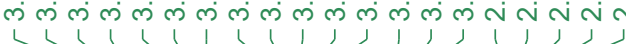




.

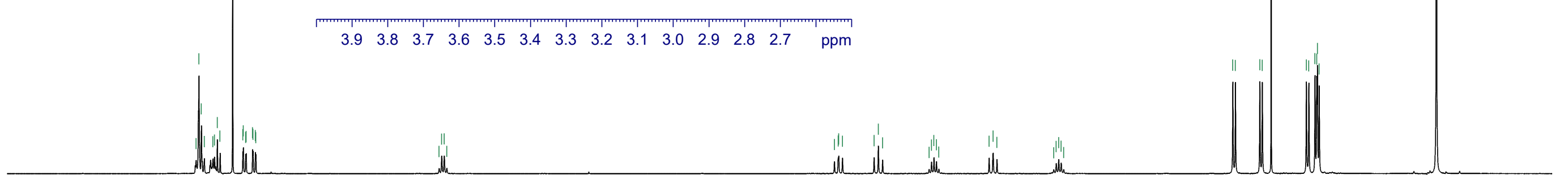

8.0

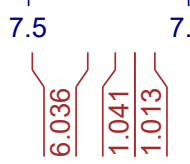

6.5

6.0

5.5

$5.0 \quad 4.5$

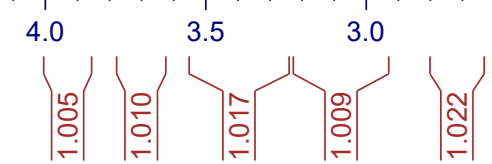

2.0

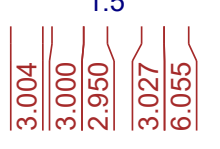

1.0

$0.5 \quad \mathrm{ppm}$ 

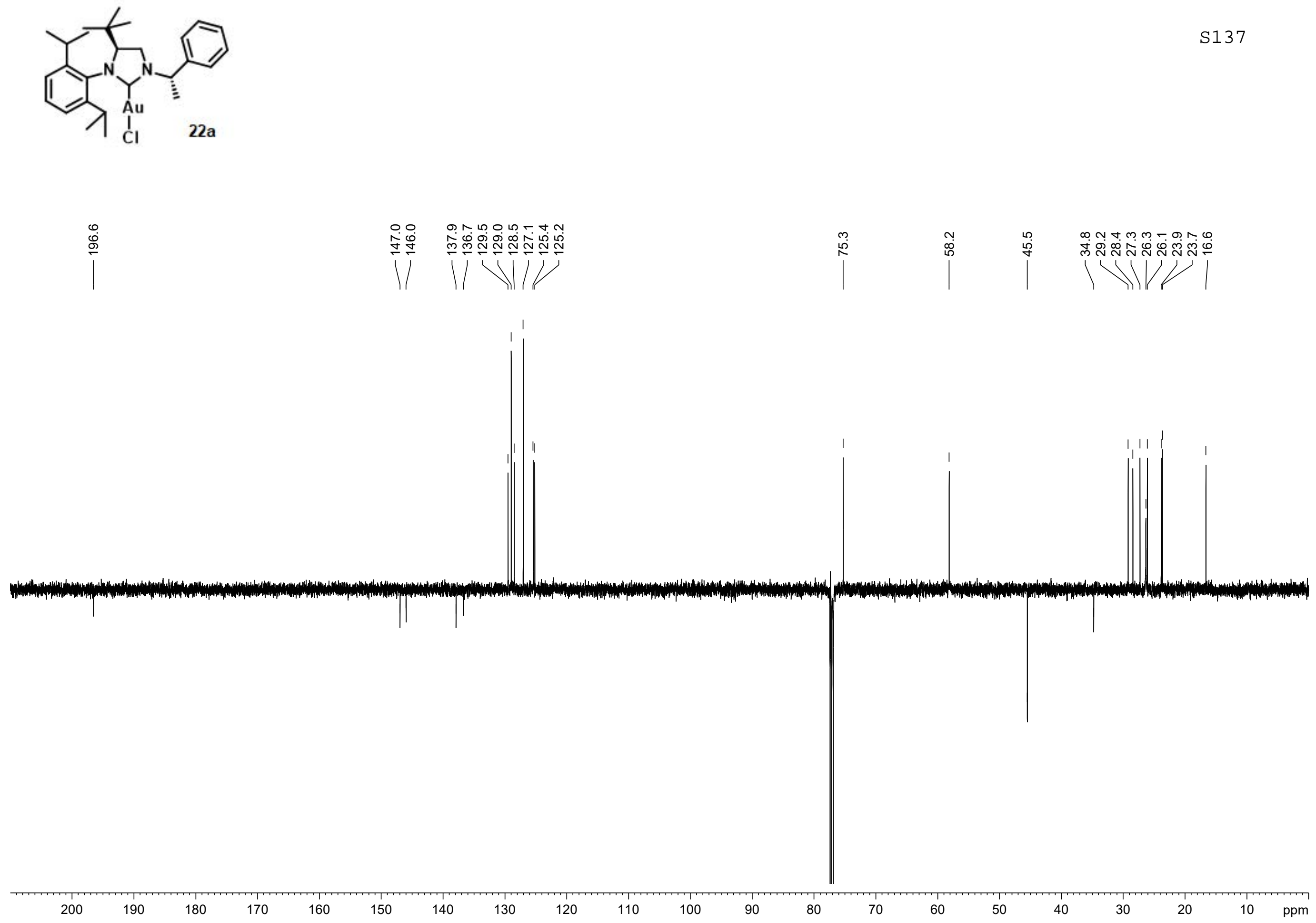


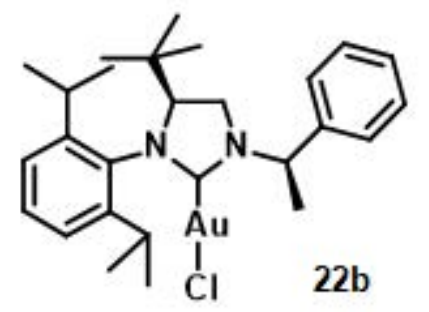

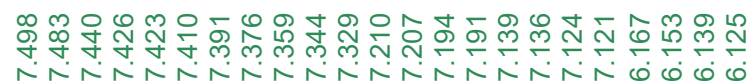

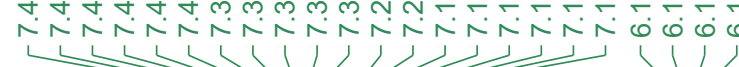

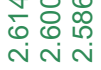

ले ले ले ले ले ले ले

VI
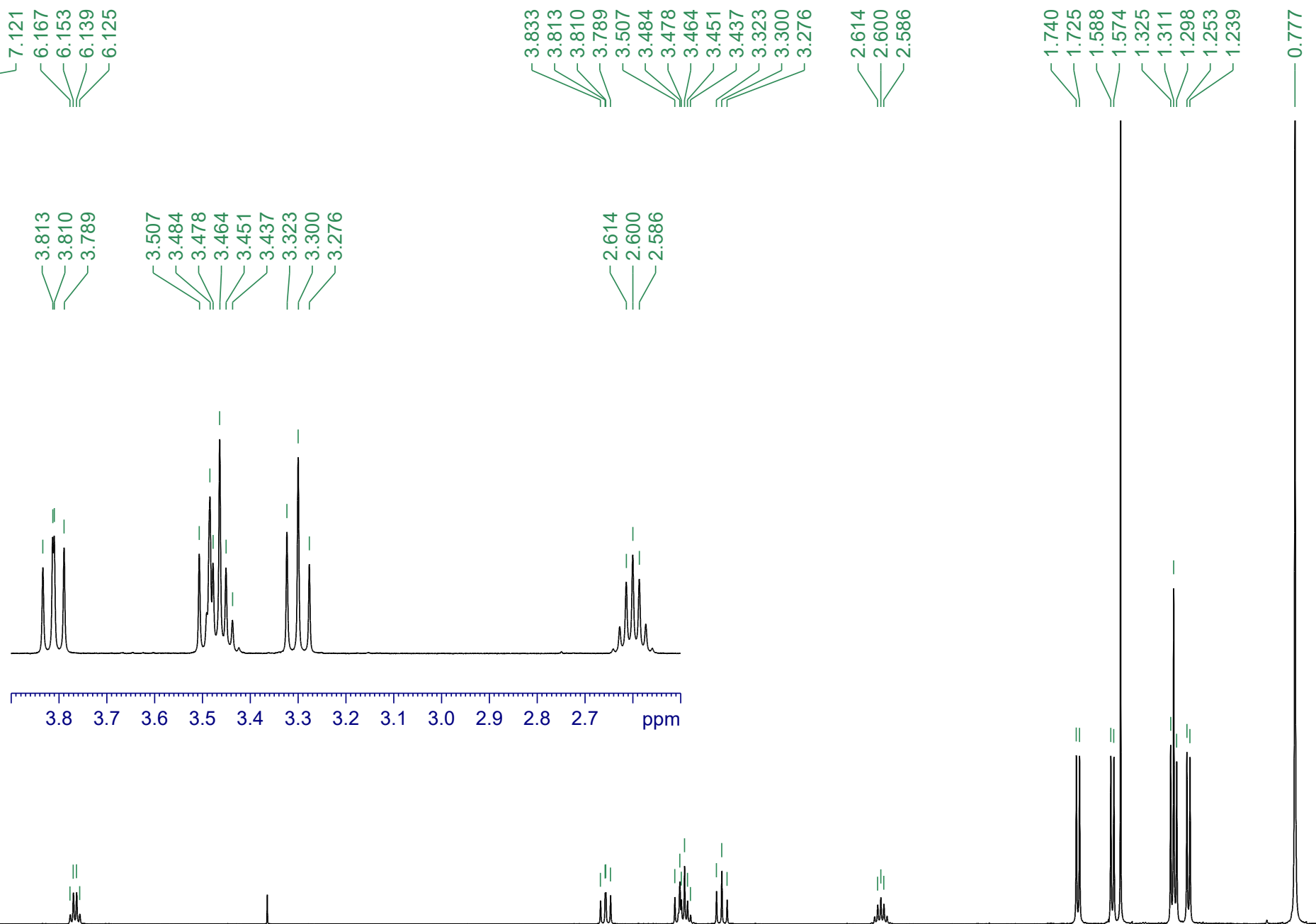

\section{응}

$\int$

Wiii

6.0

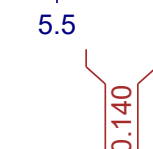

5.0

4.5

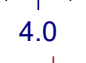

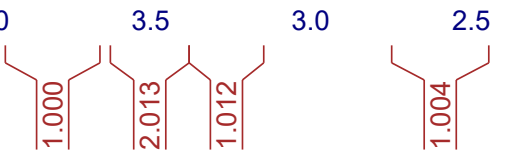

2.0

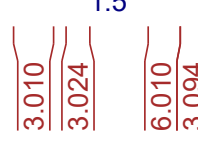

1.0

$0.5 \mathrm{ppm}$ 


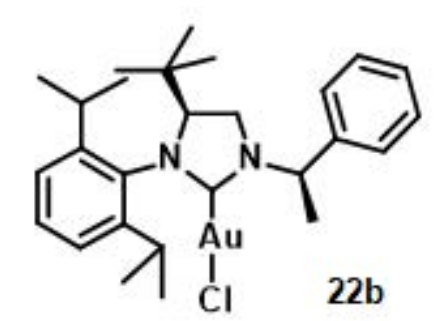

ণ্்
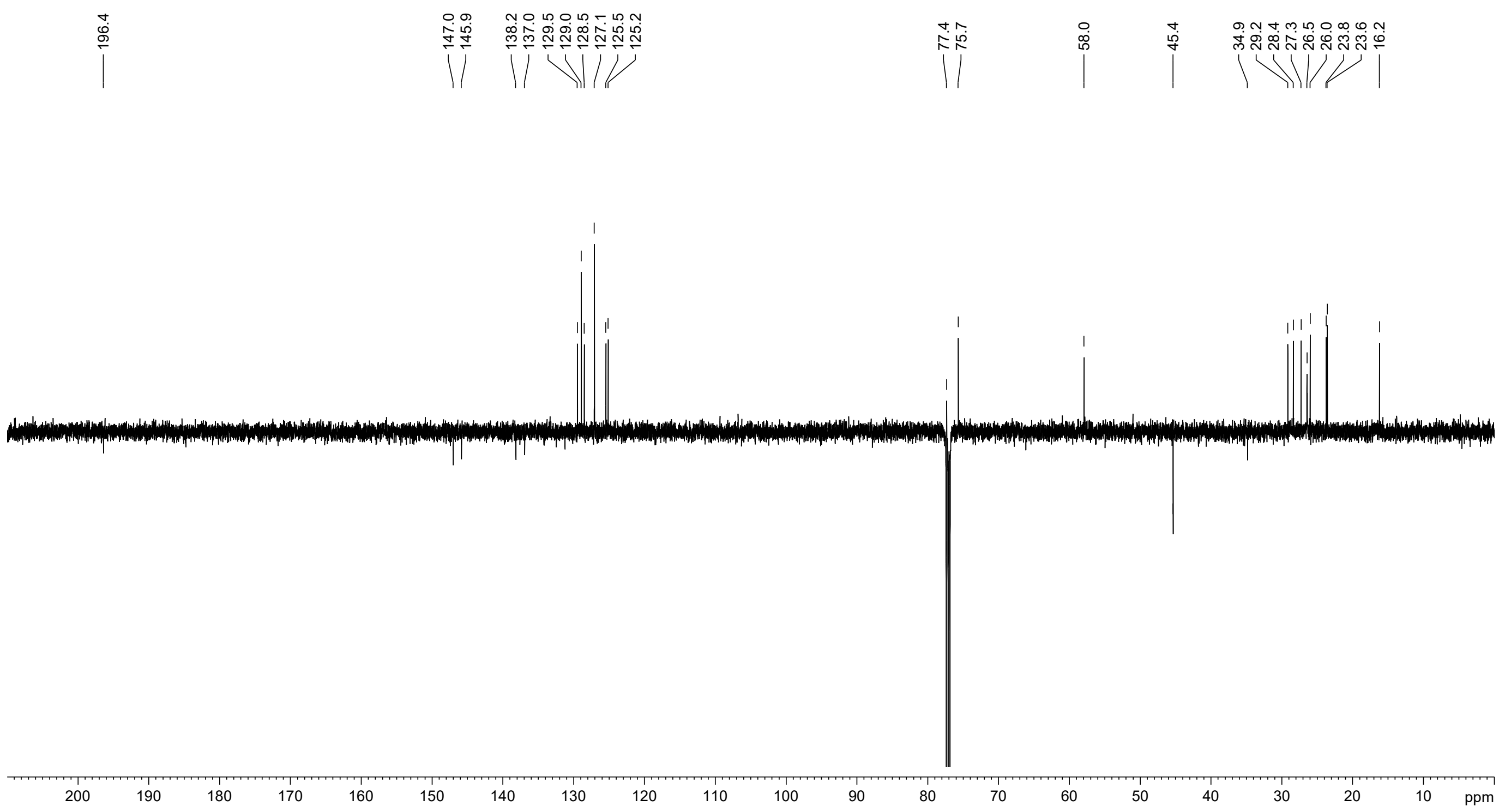




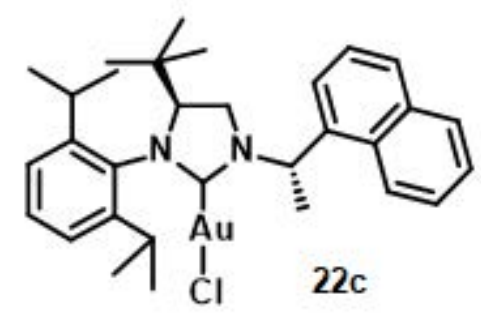

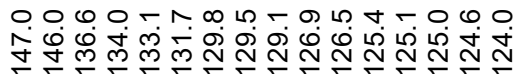

I WWV

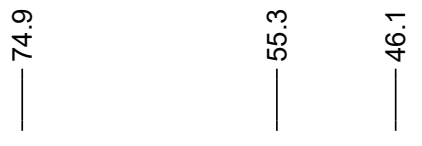

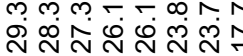

11

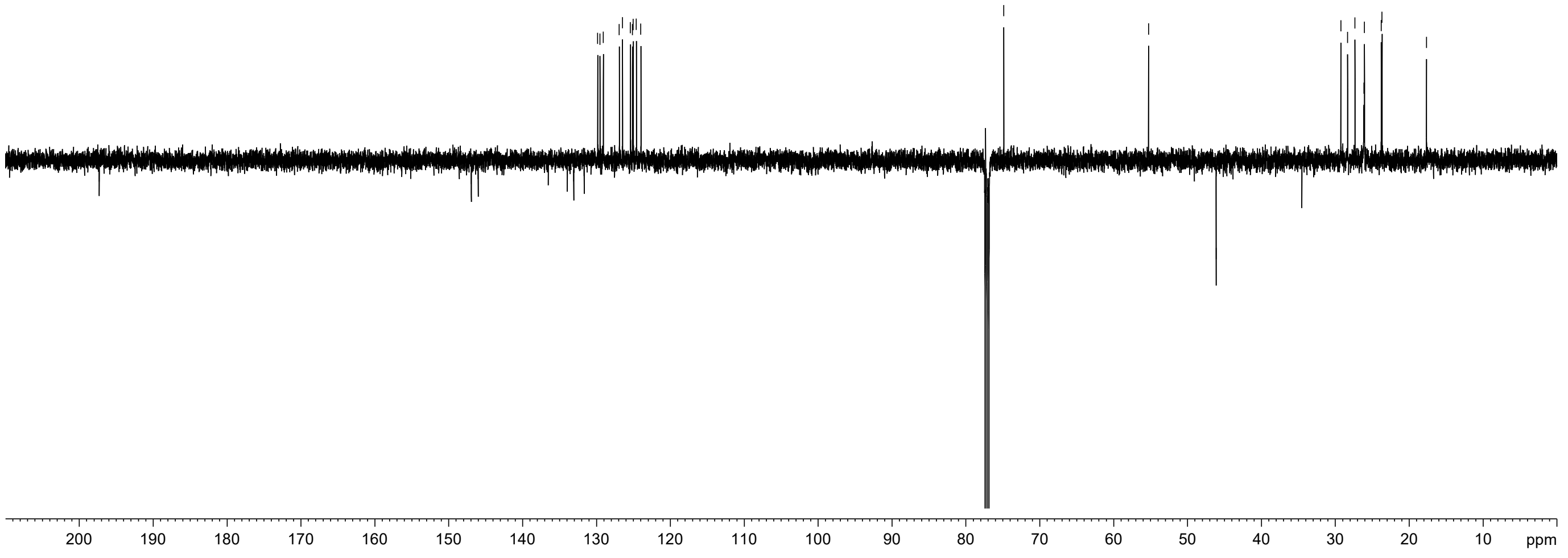




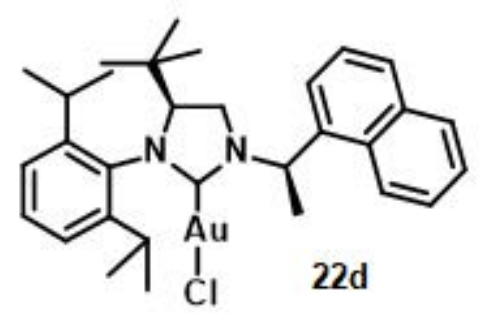



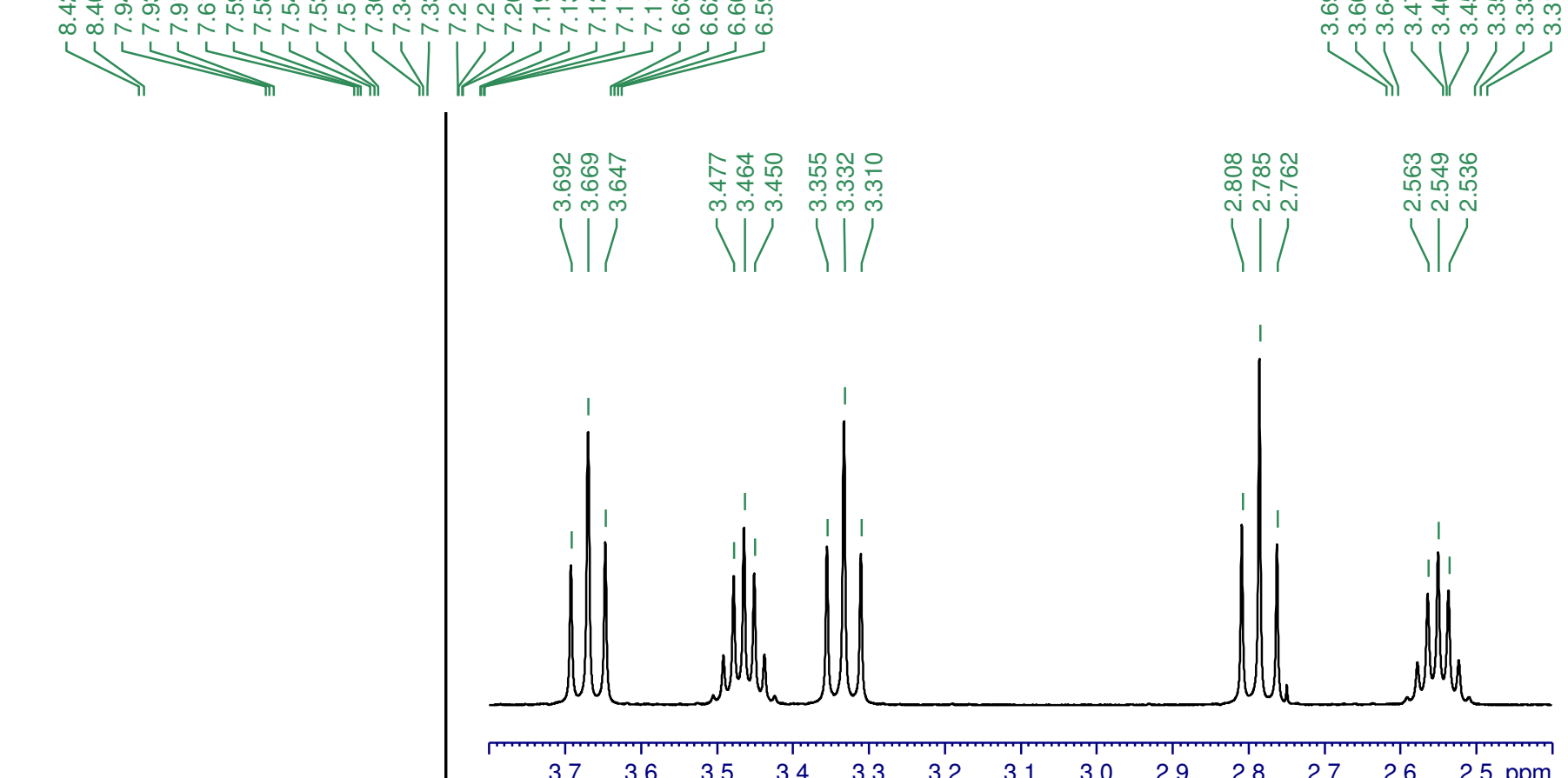

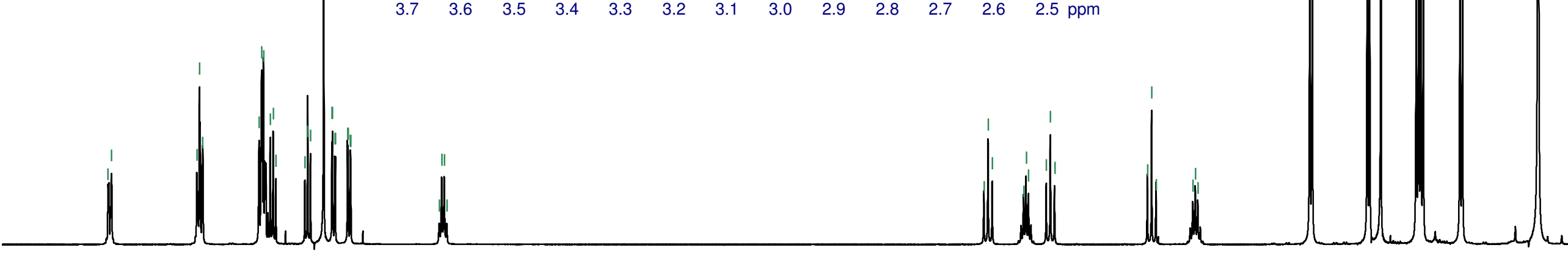

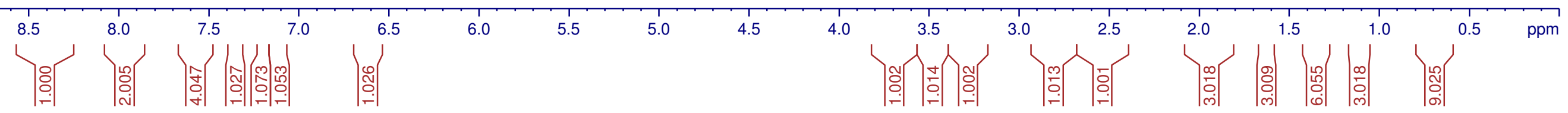



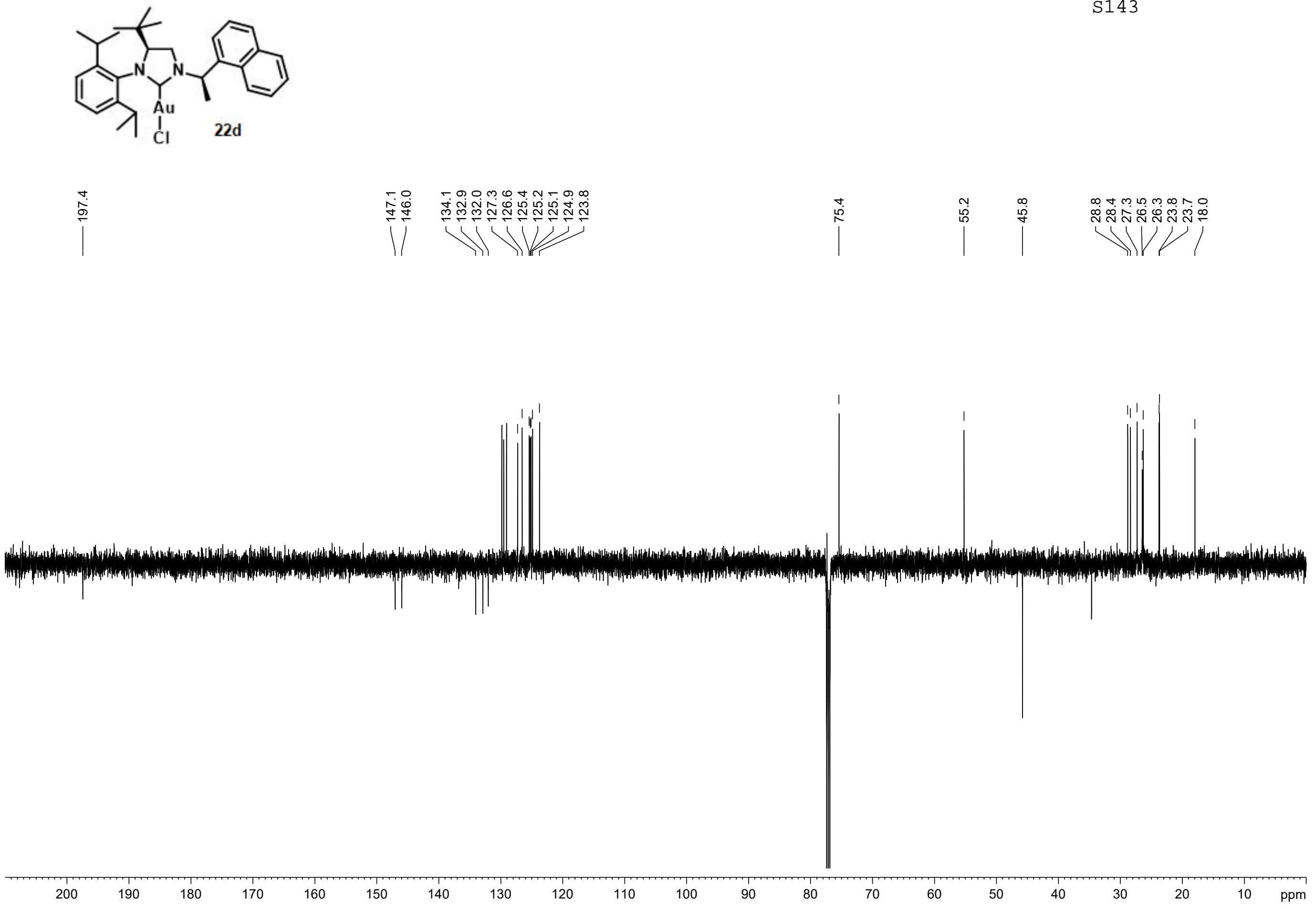

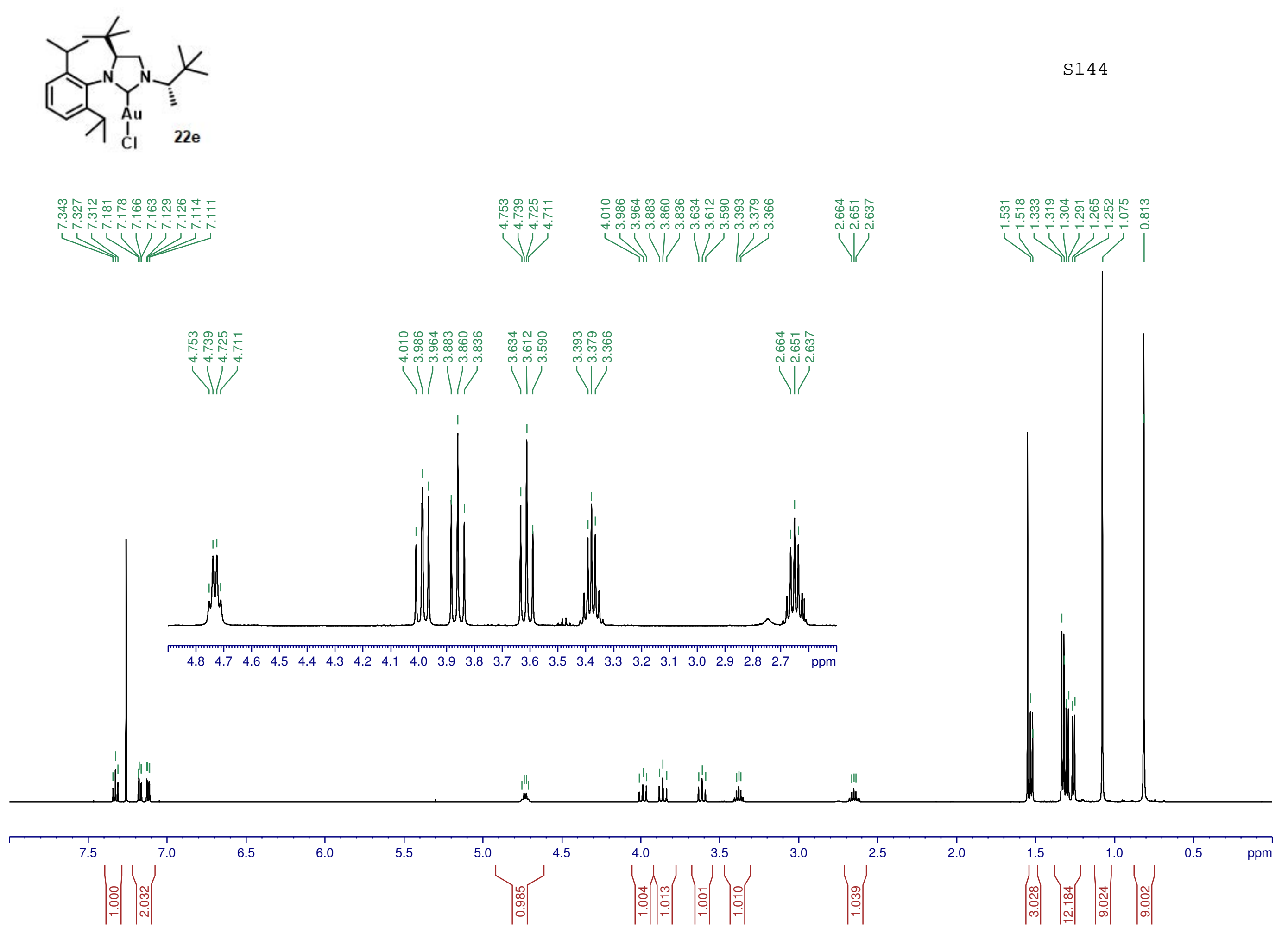


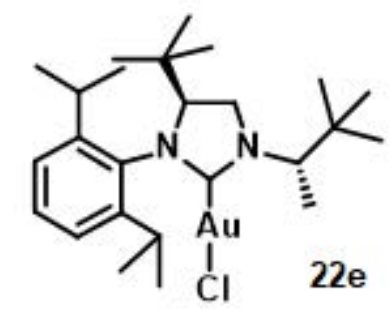

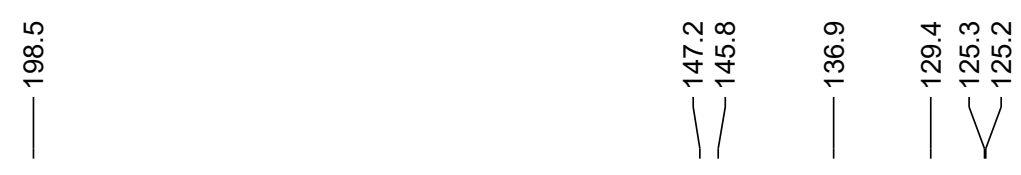

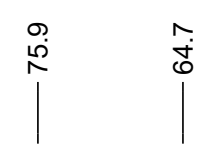

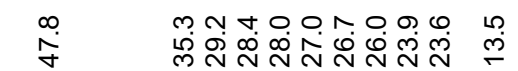

WW/

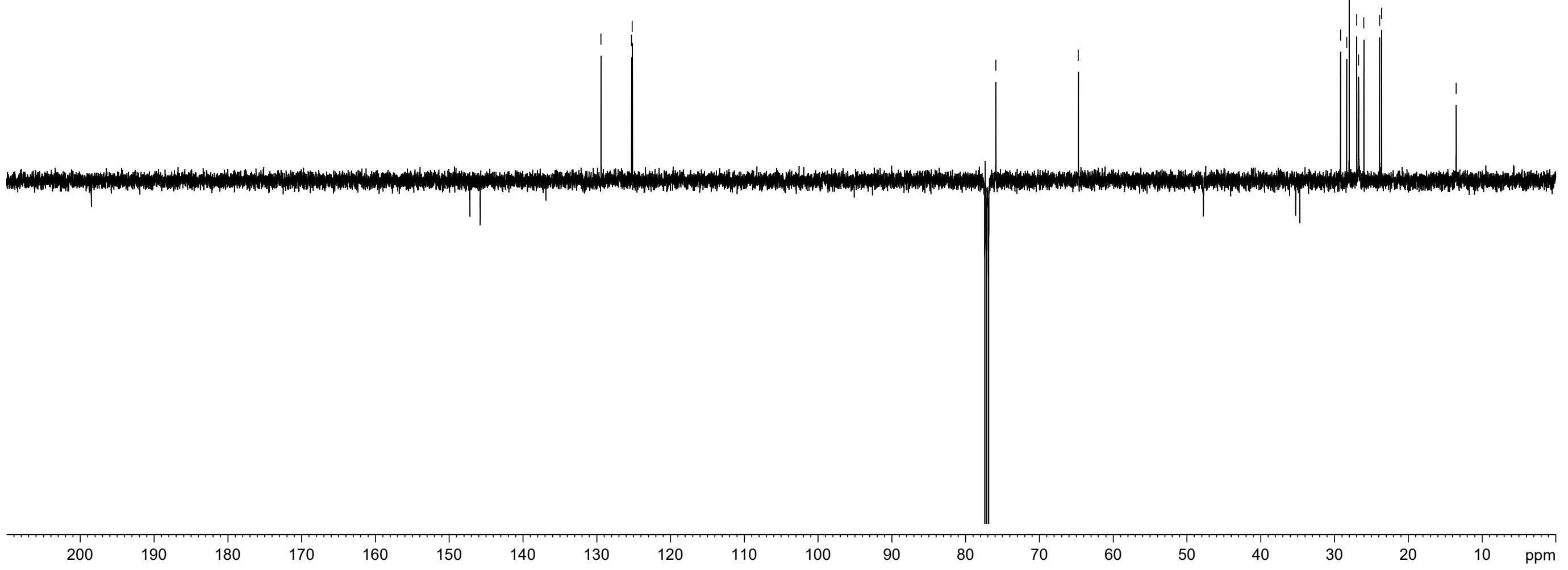



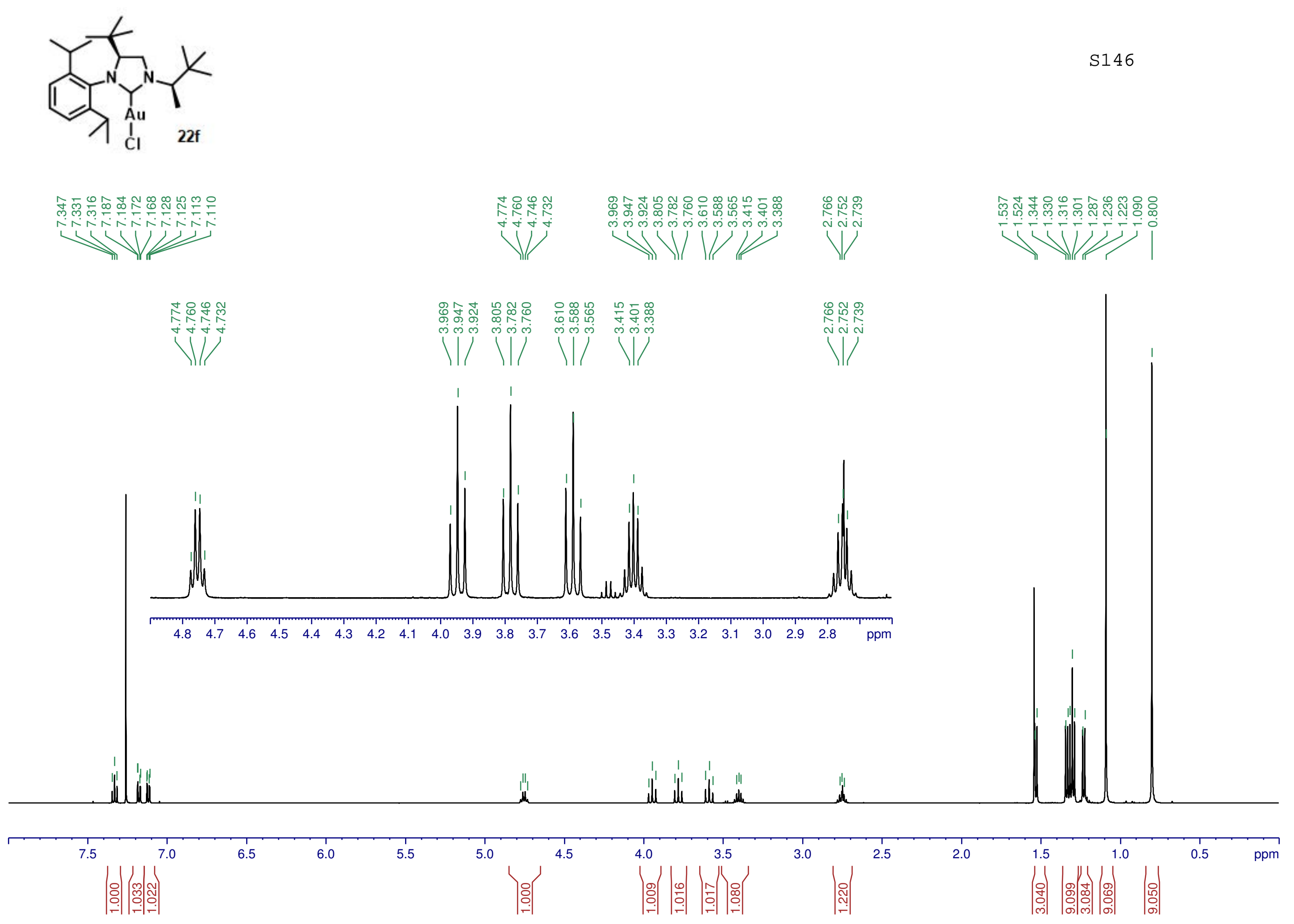


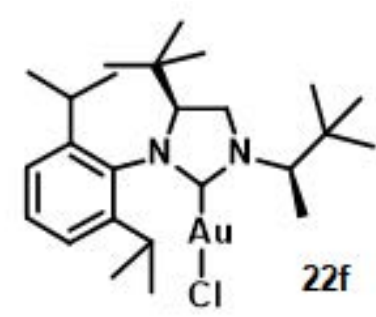

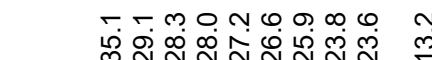
\lceil

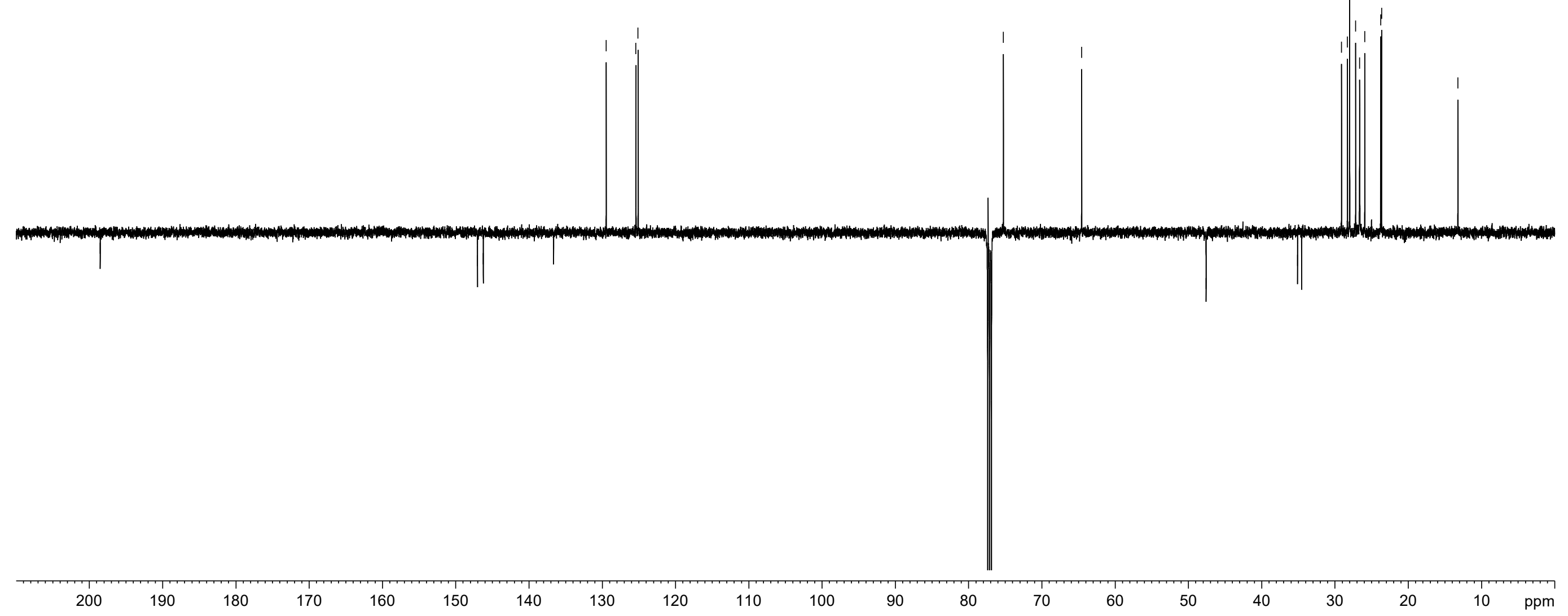




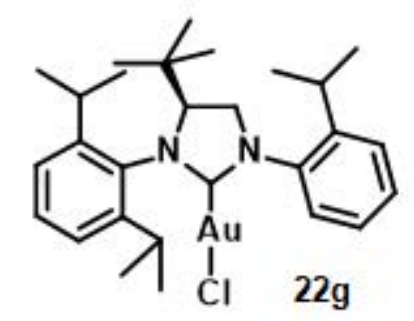

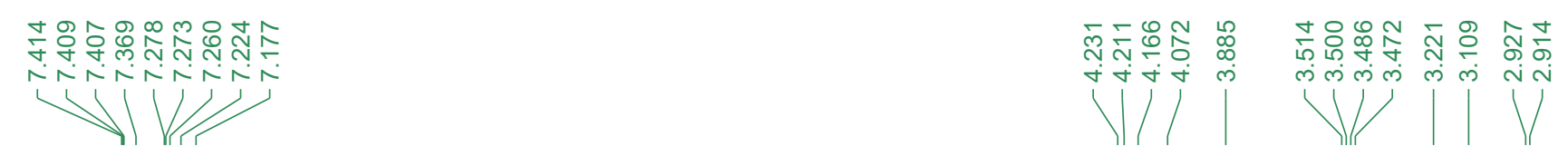

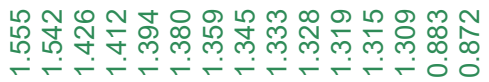

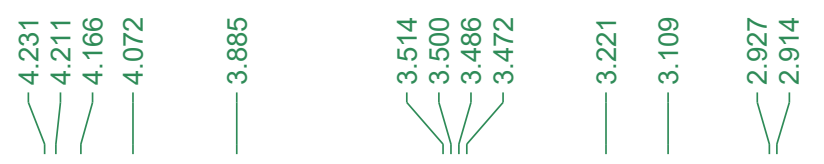
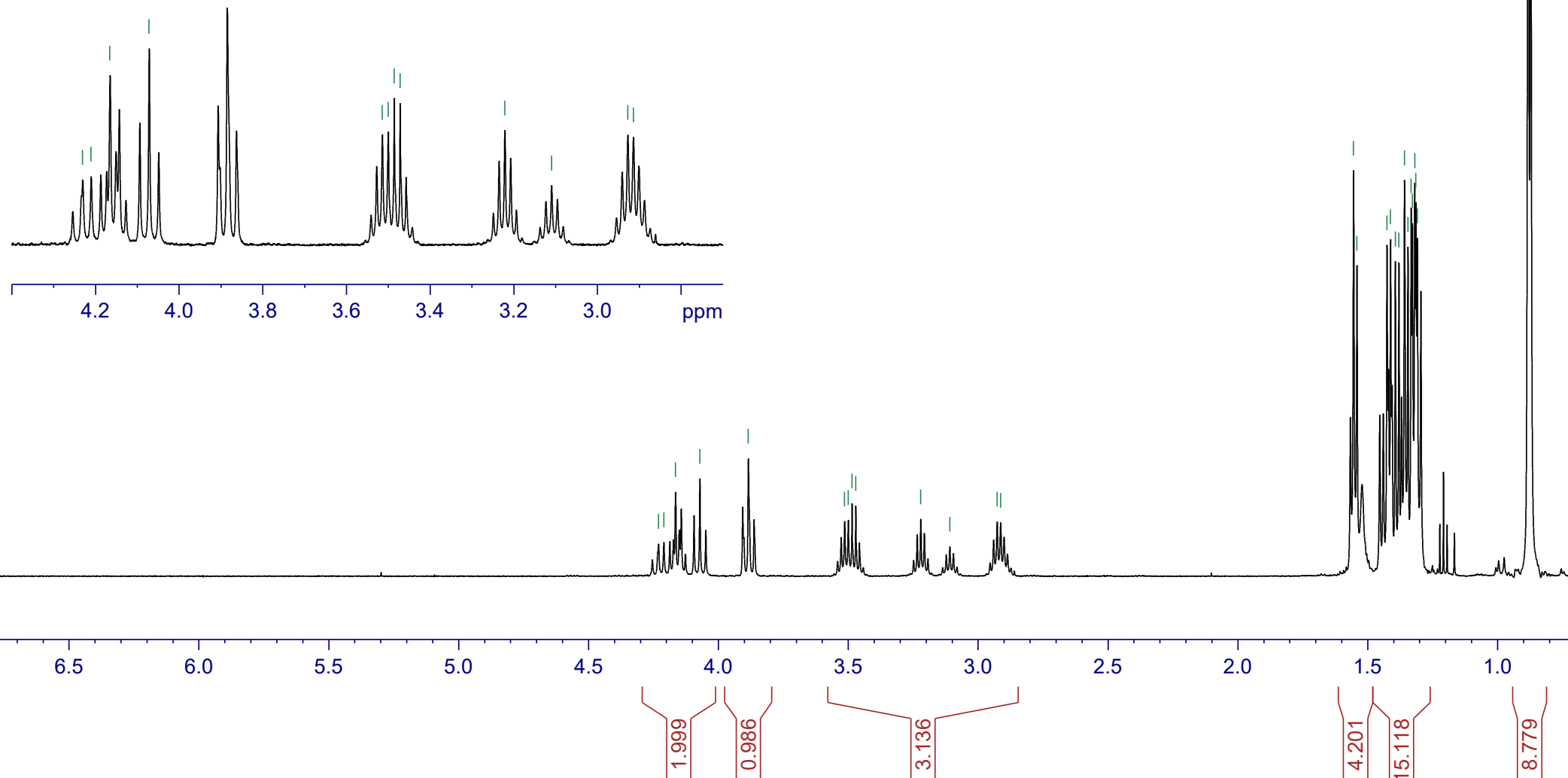

2.5

2.0

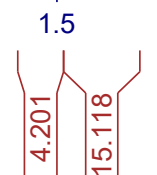




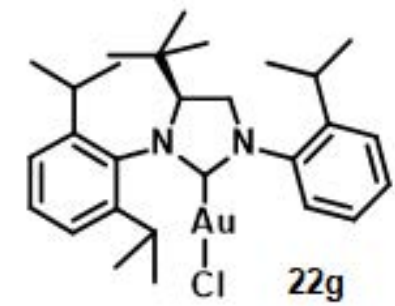




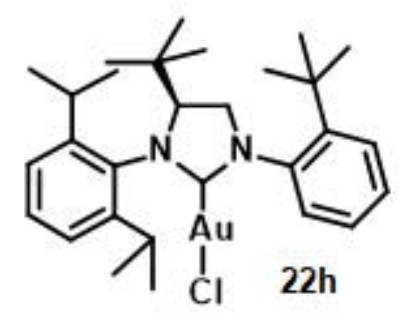

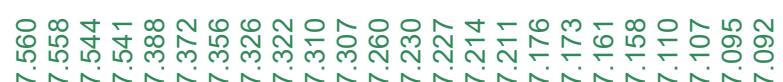



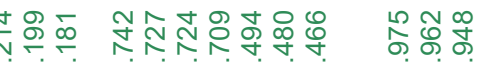

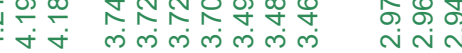

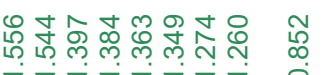

7.5

7.0

6.5

6.0

5.0

4.5

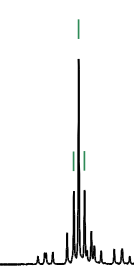




2.5
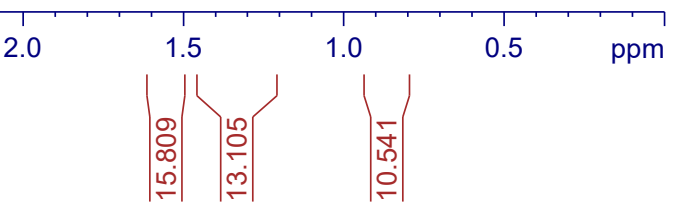


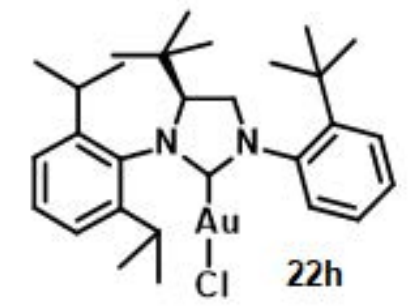

옴

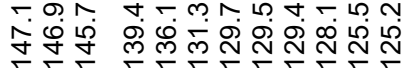
VT Tivit

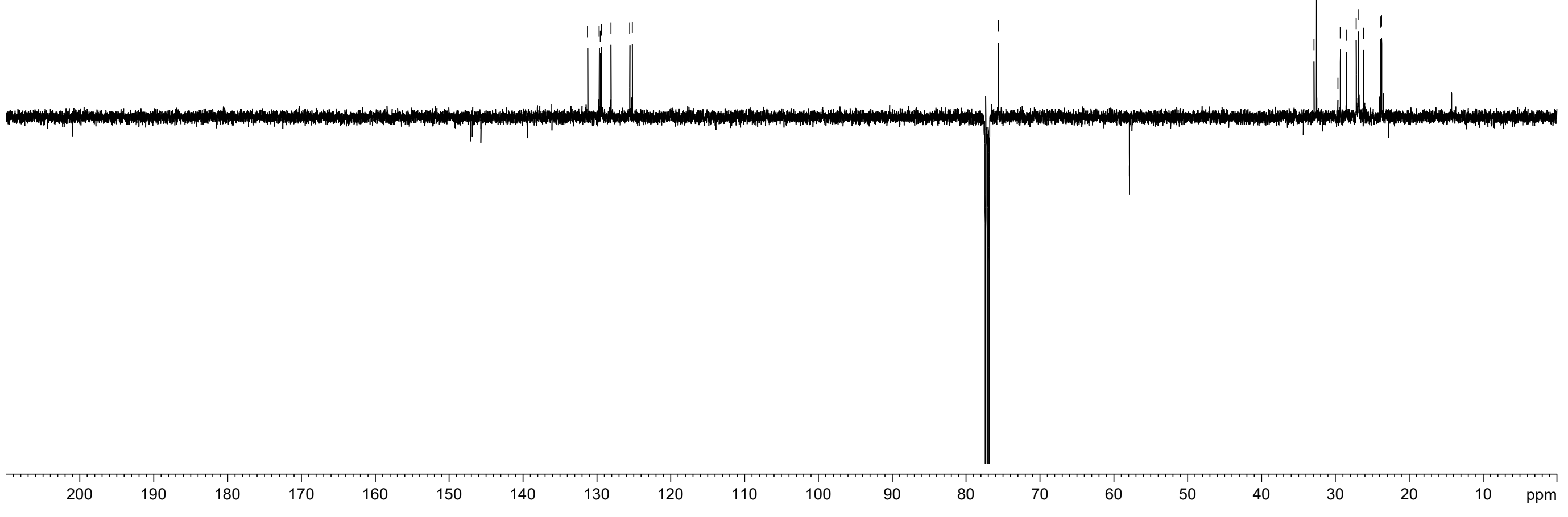




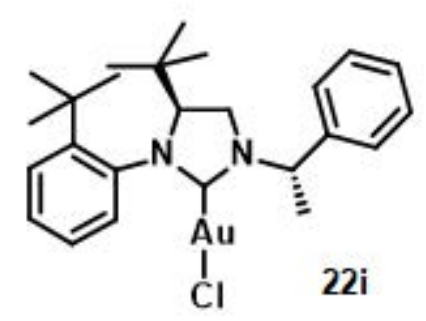

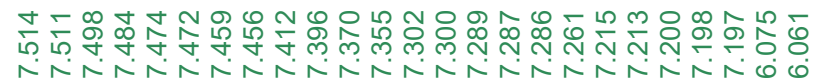

$\longrightarrow$

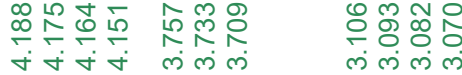

$\sqrt[V]{\text { if juj }}$

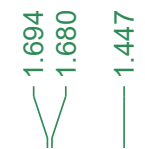

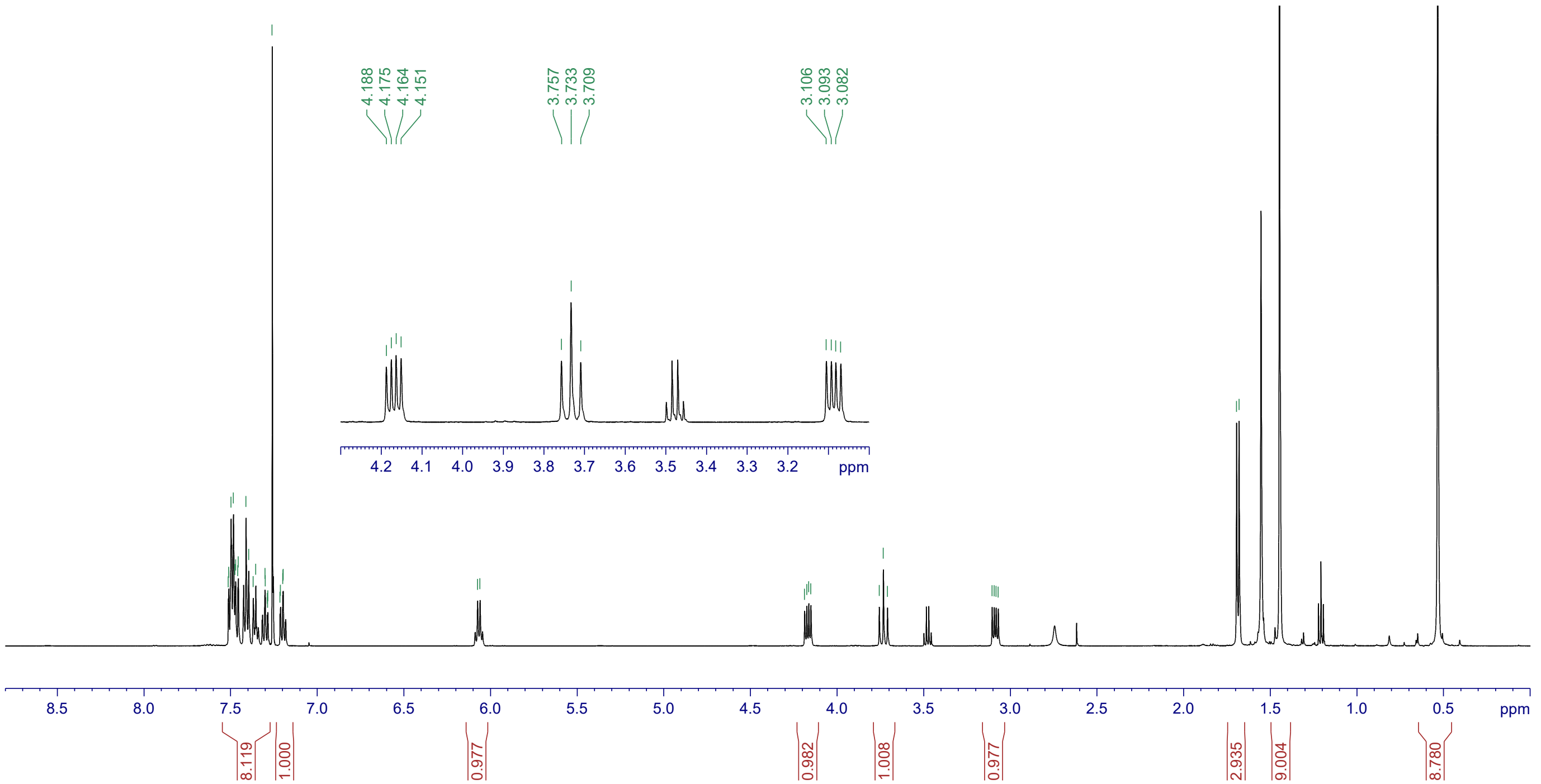



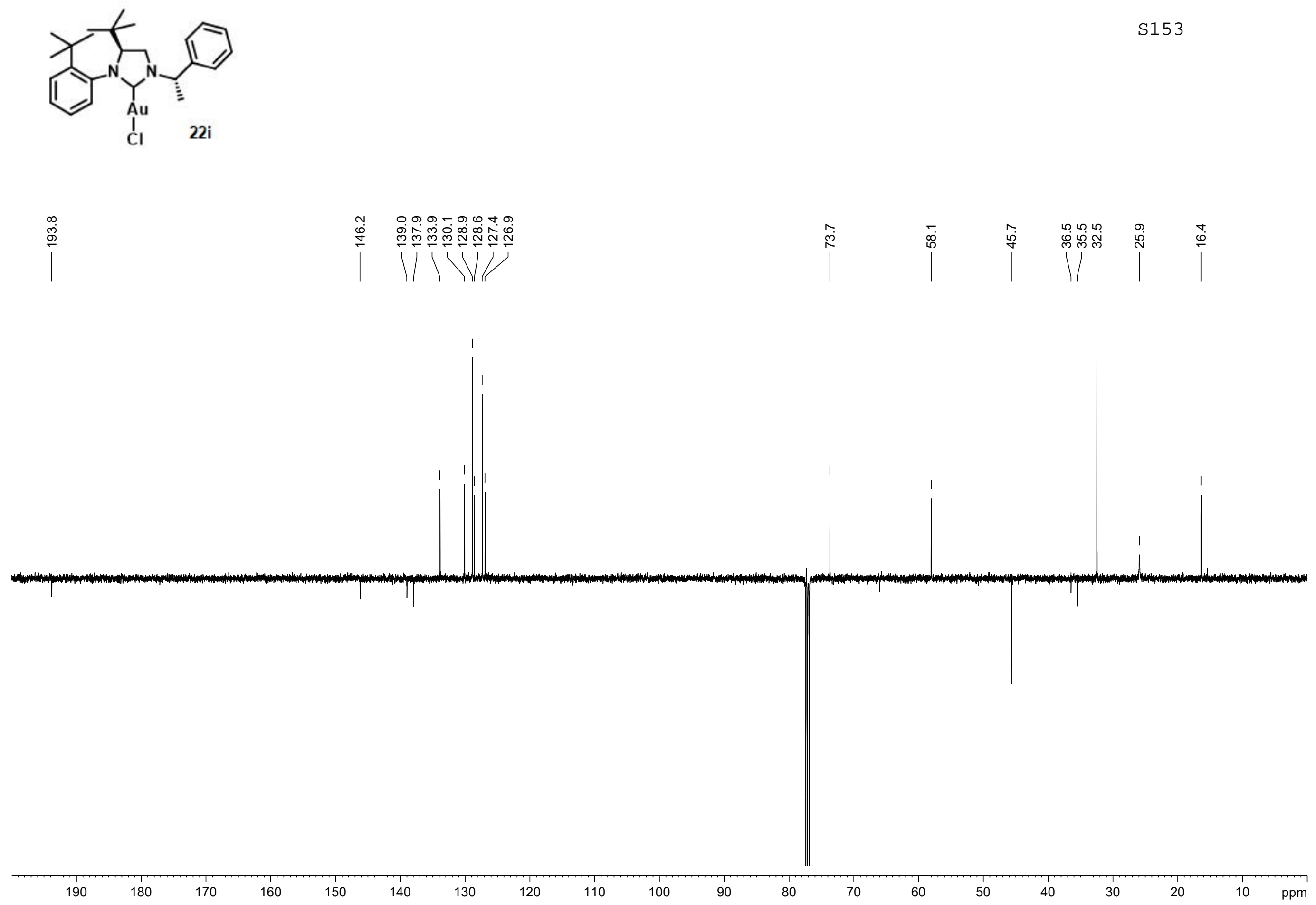


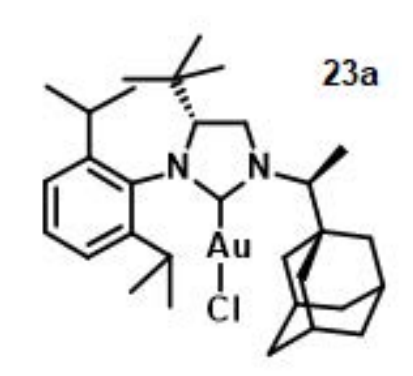

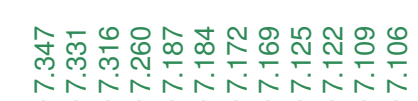

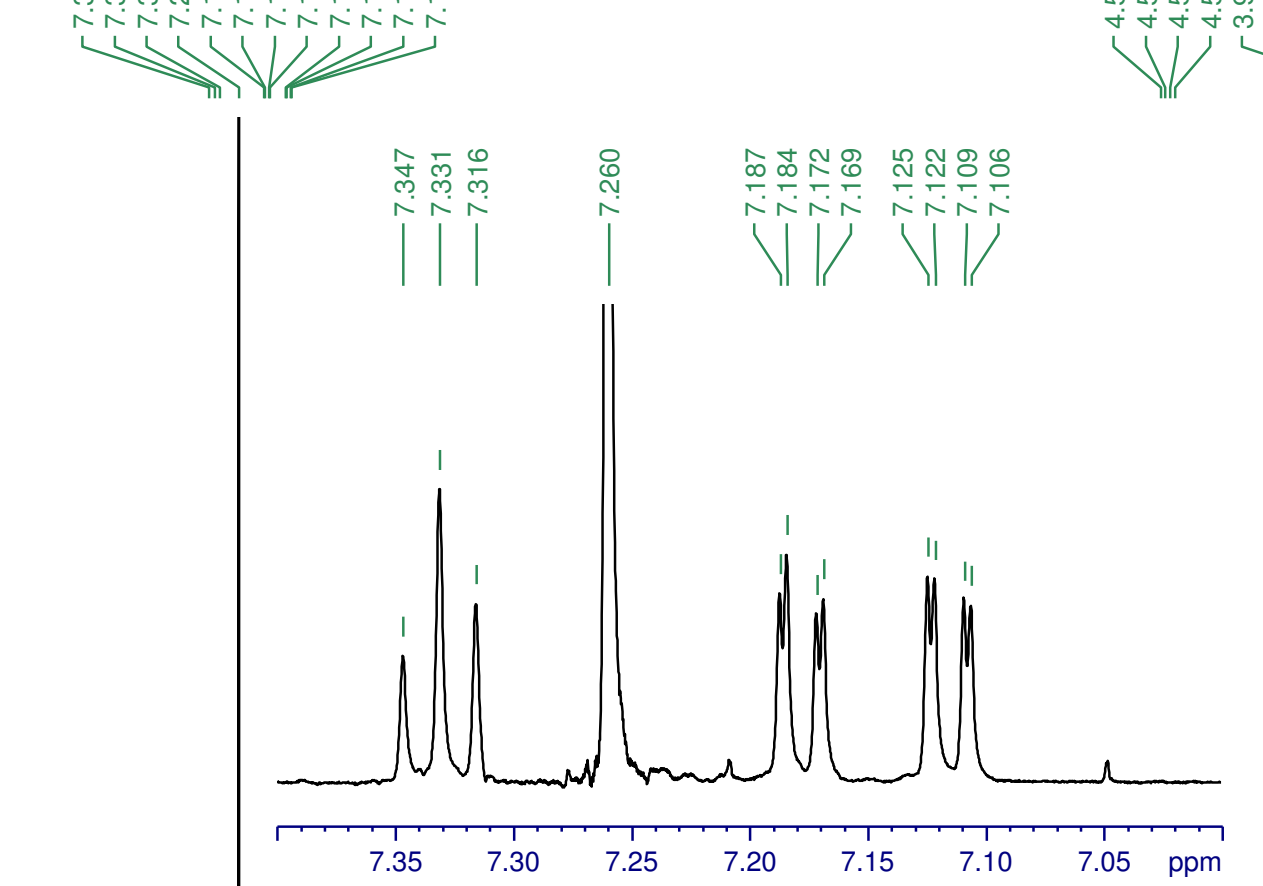

s154

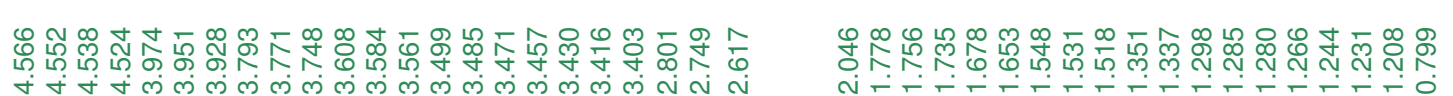

V.

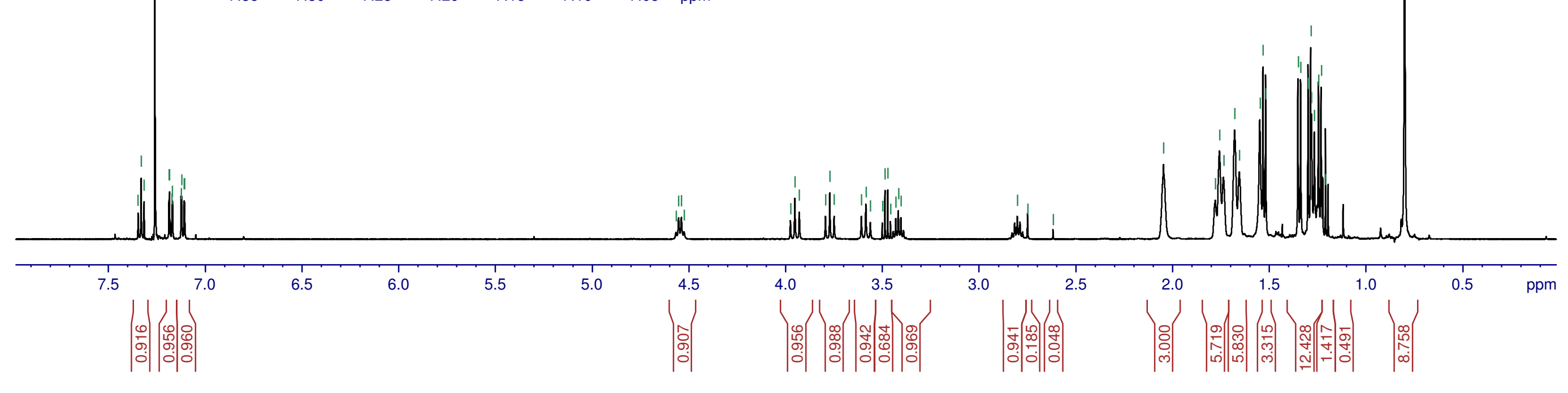



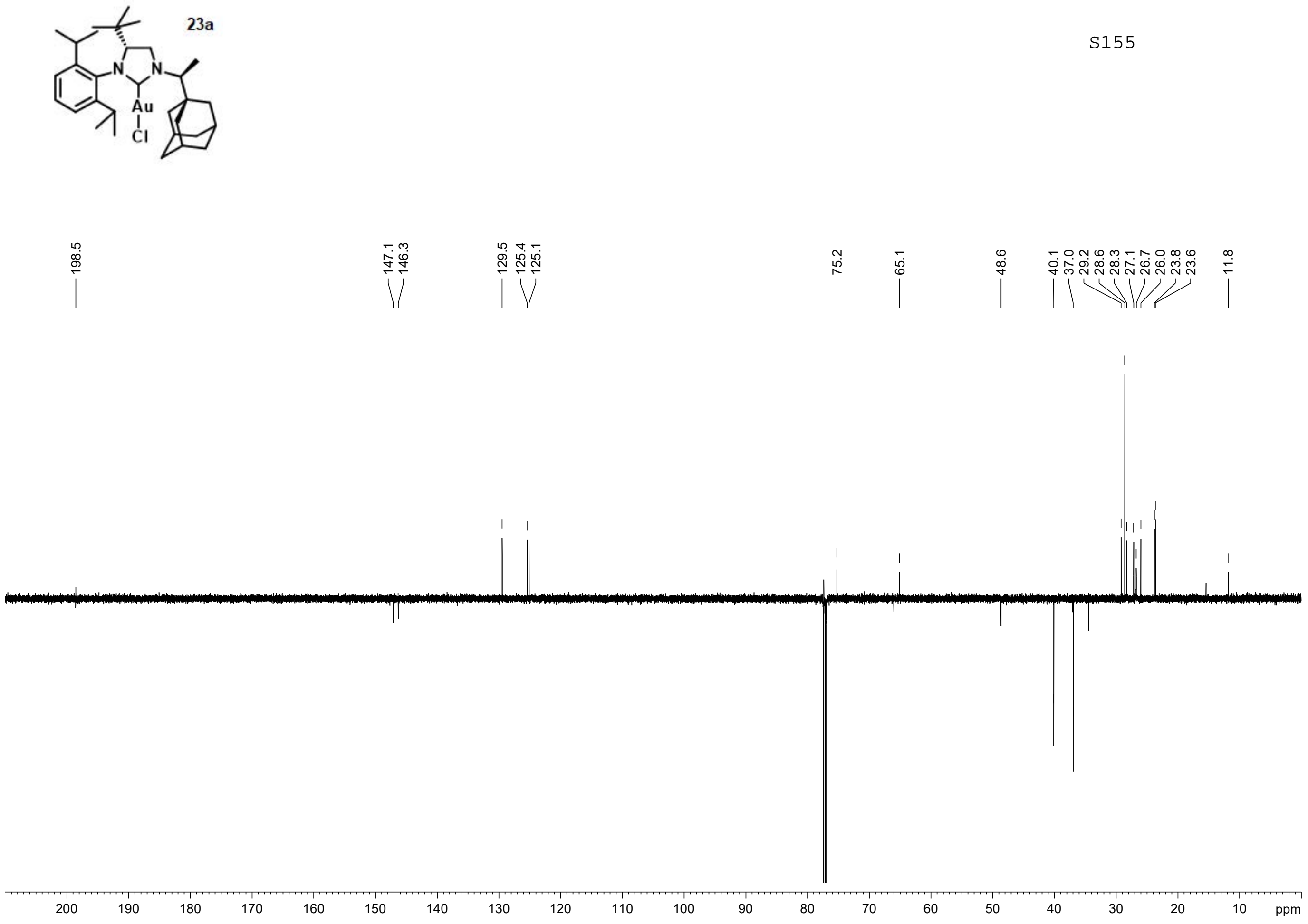

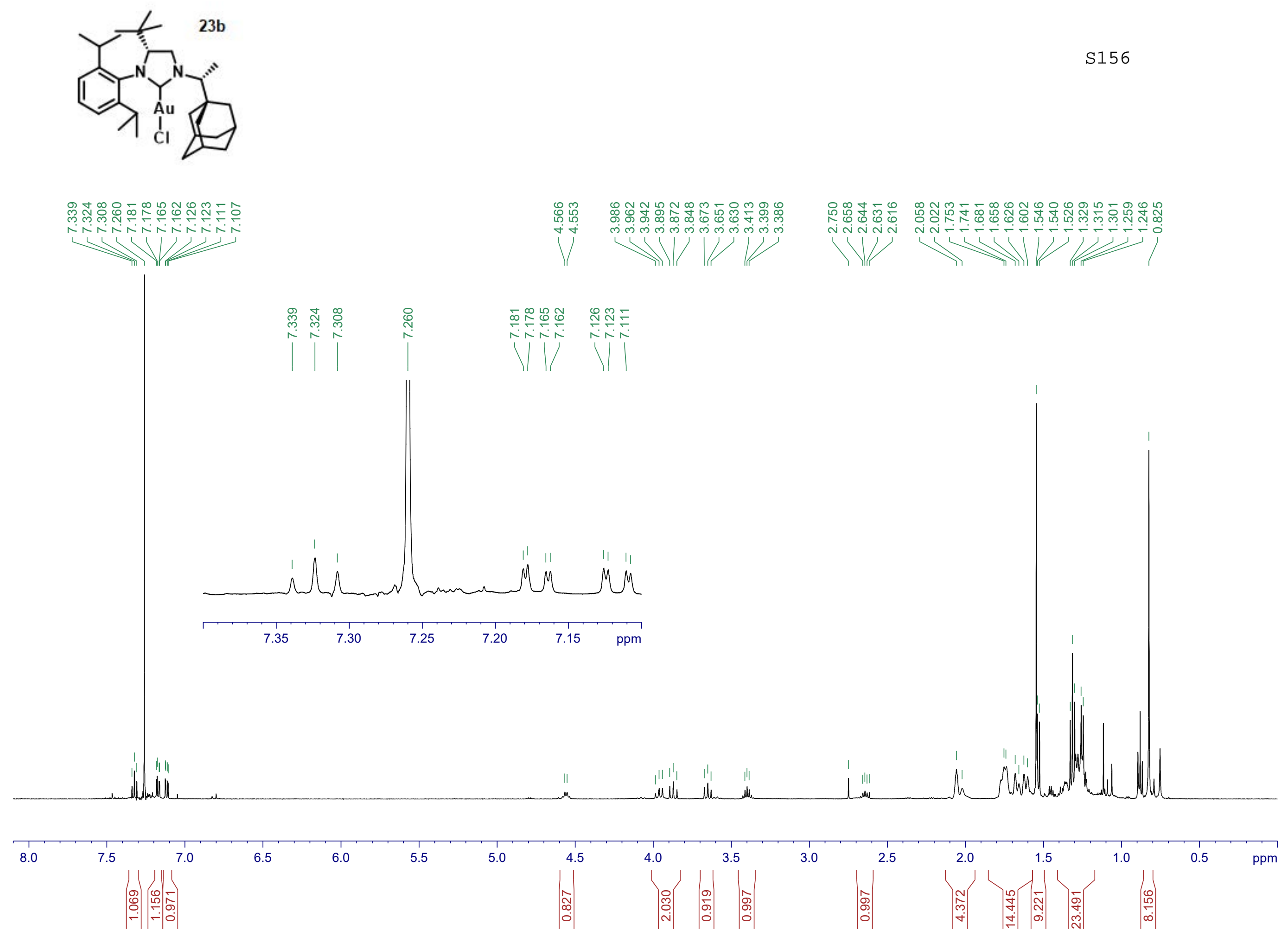

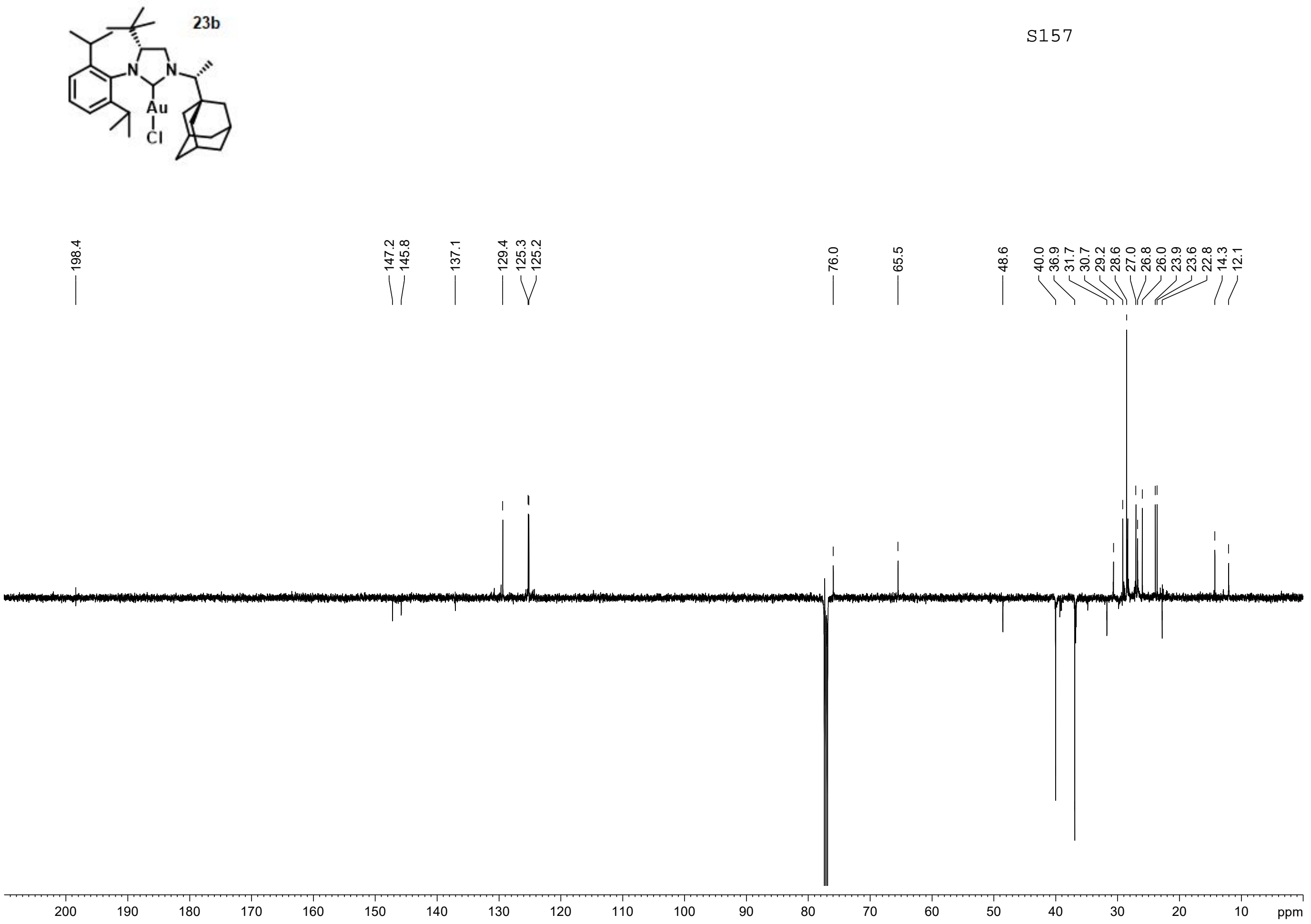


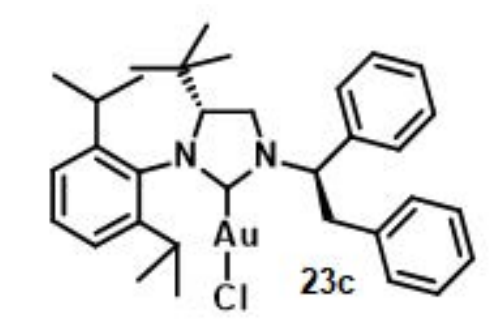

品

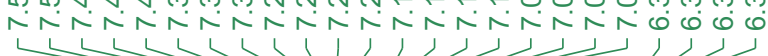

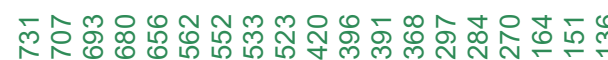

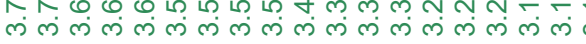
$1<1$ (ग)

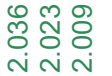
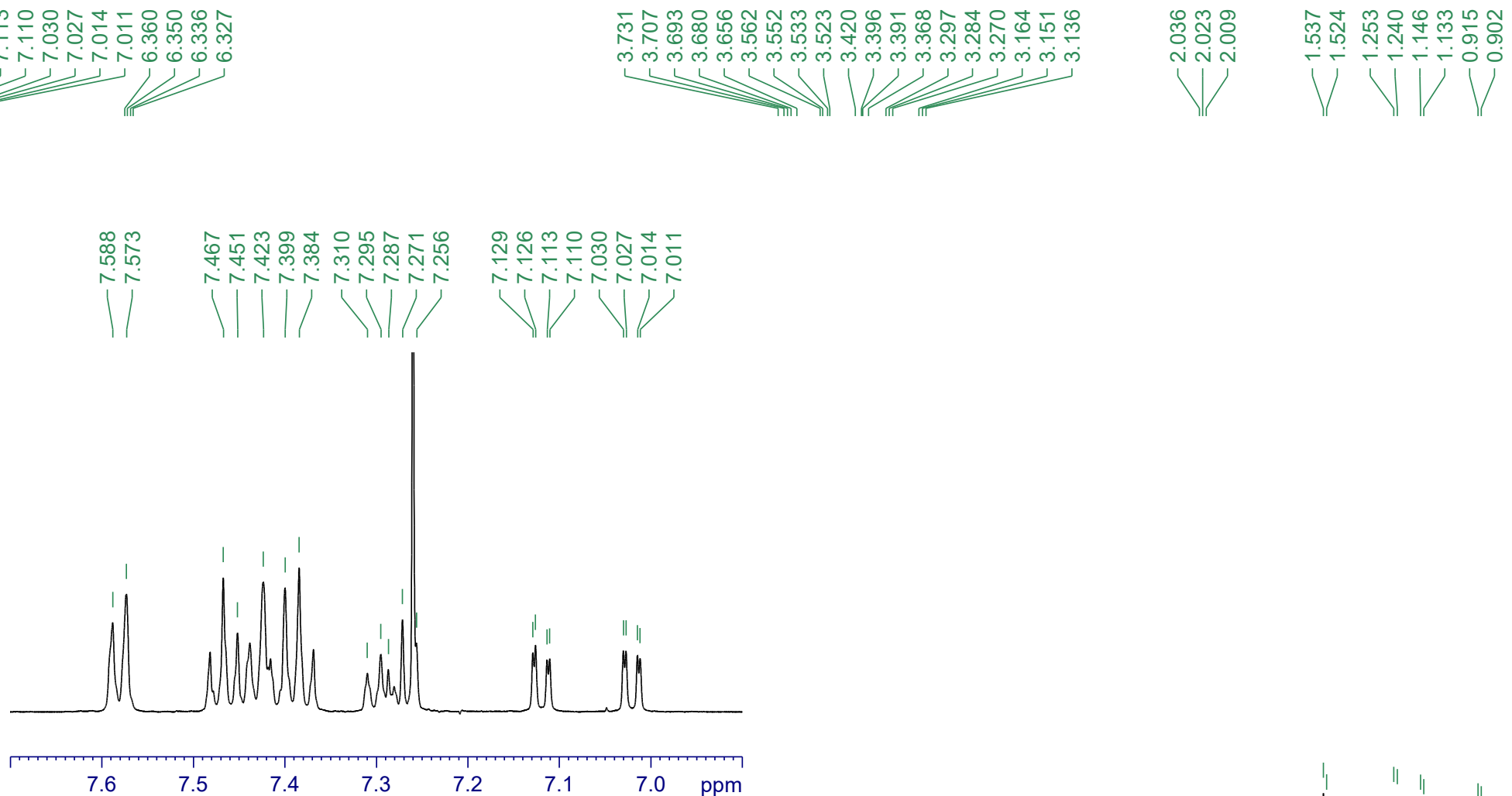

11, hinut 

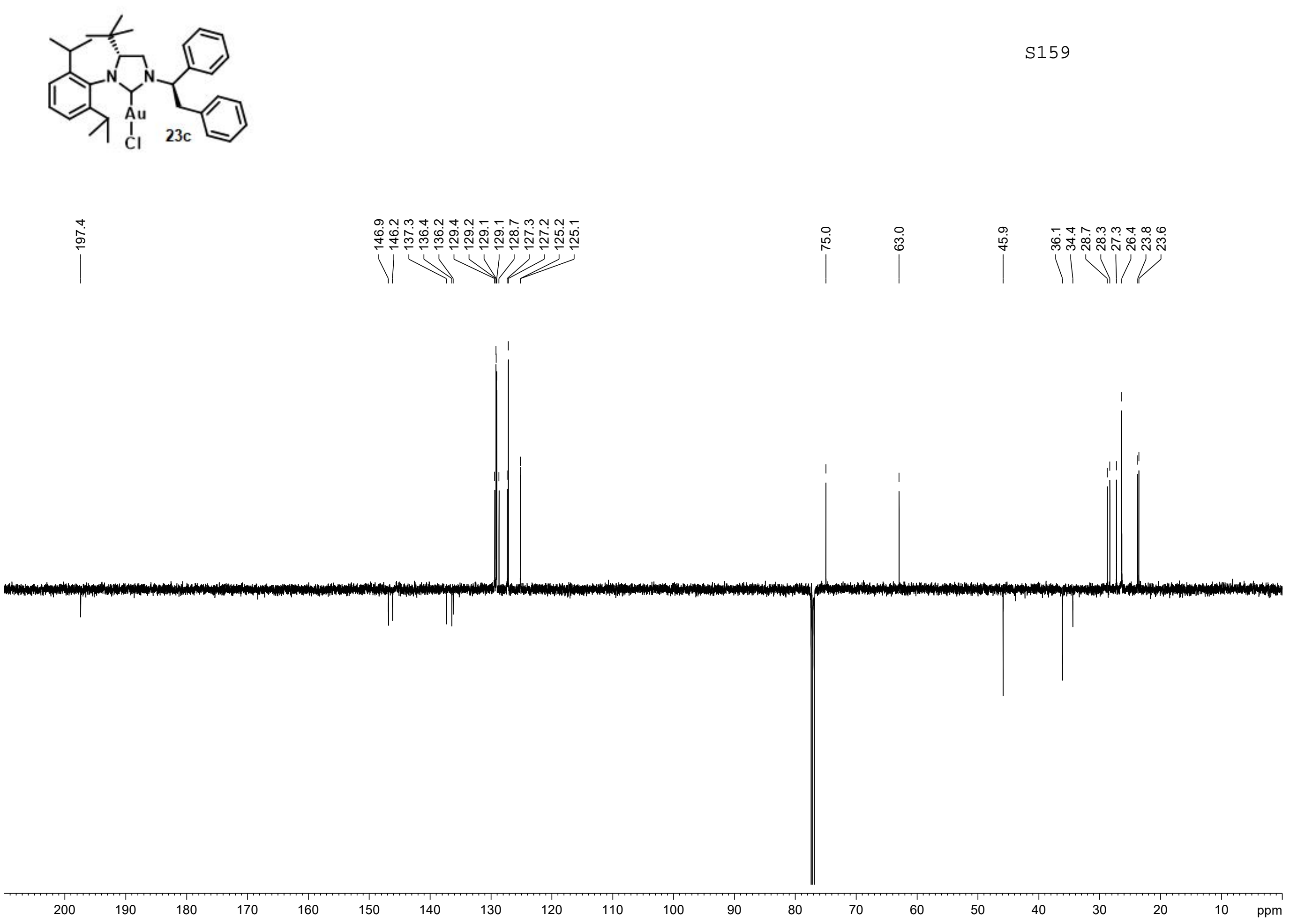


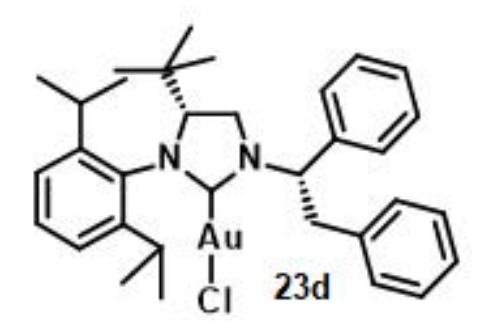

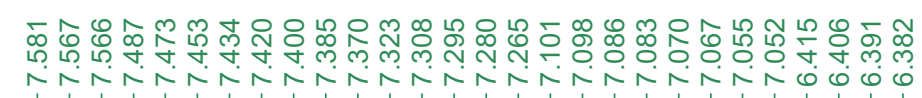

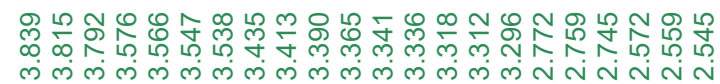

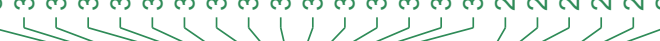
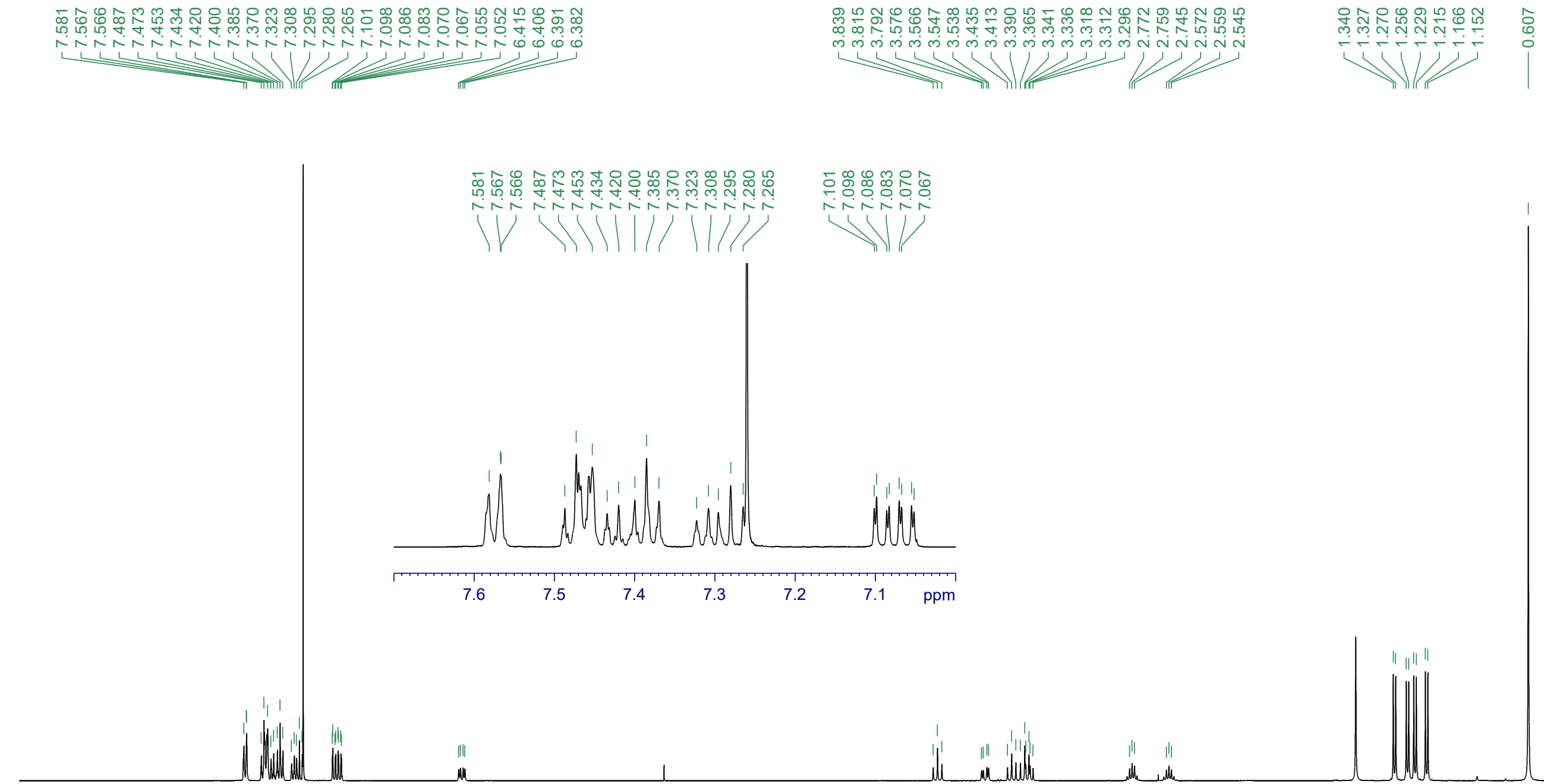

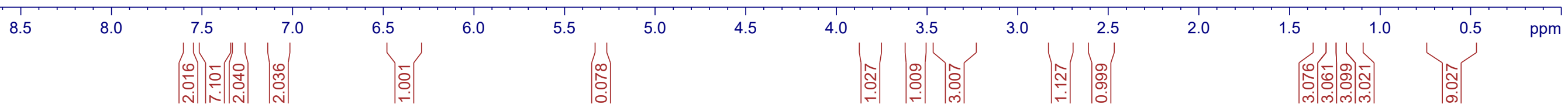



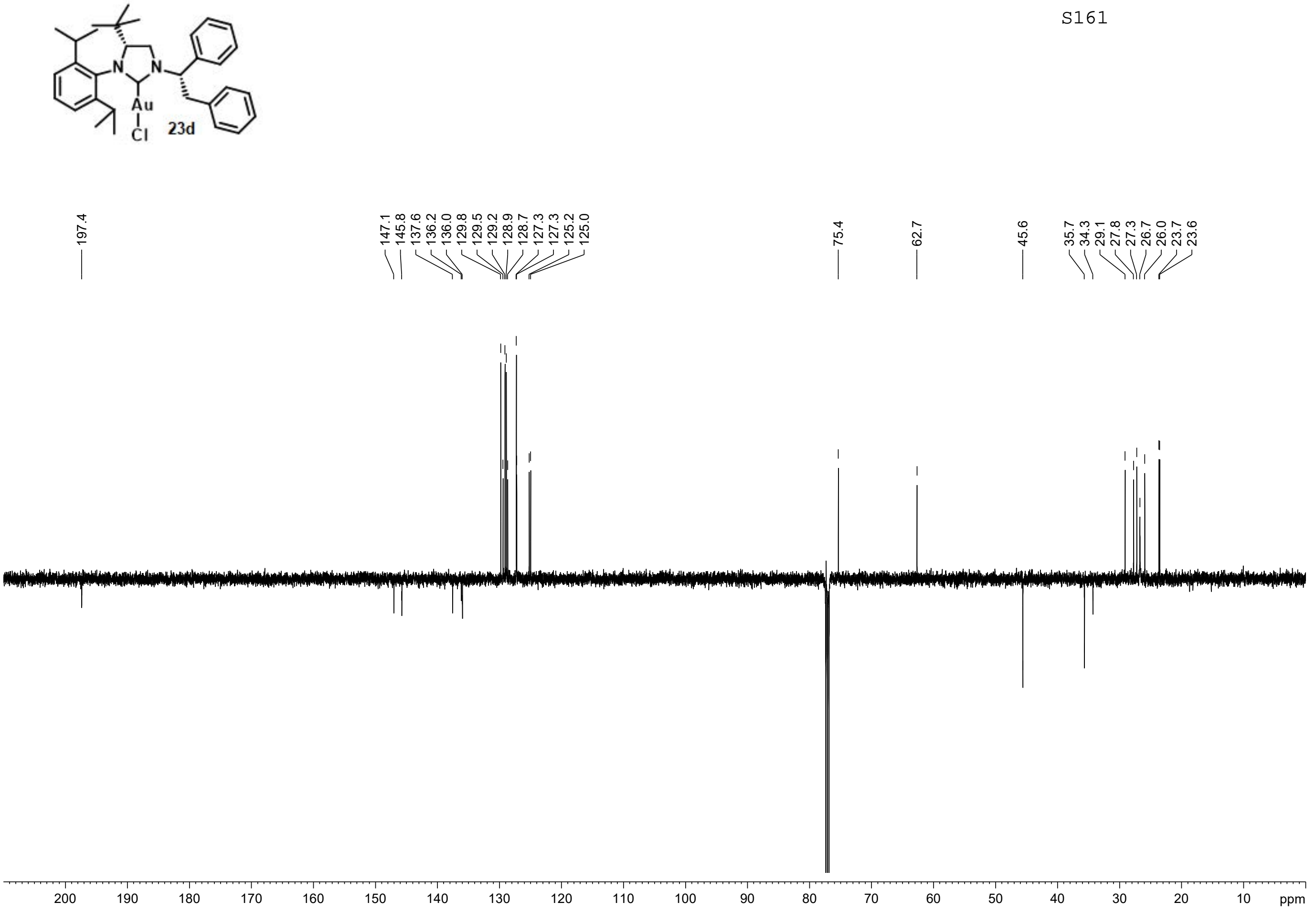

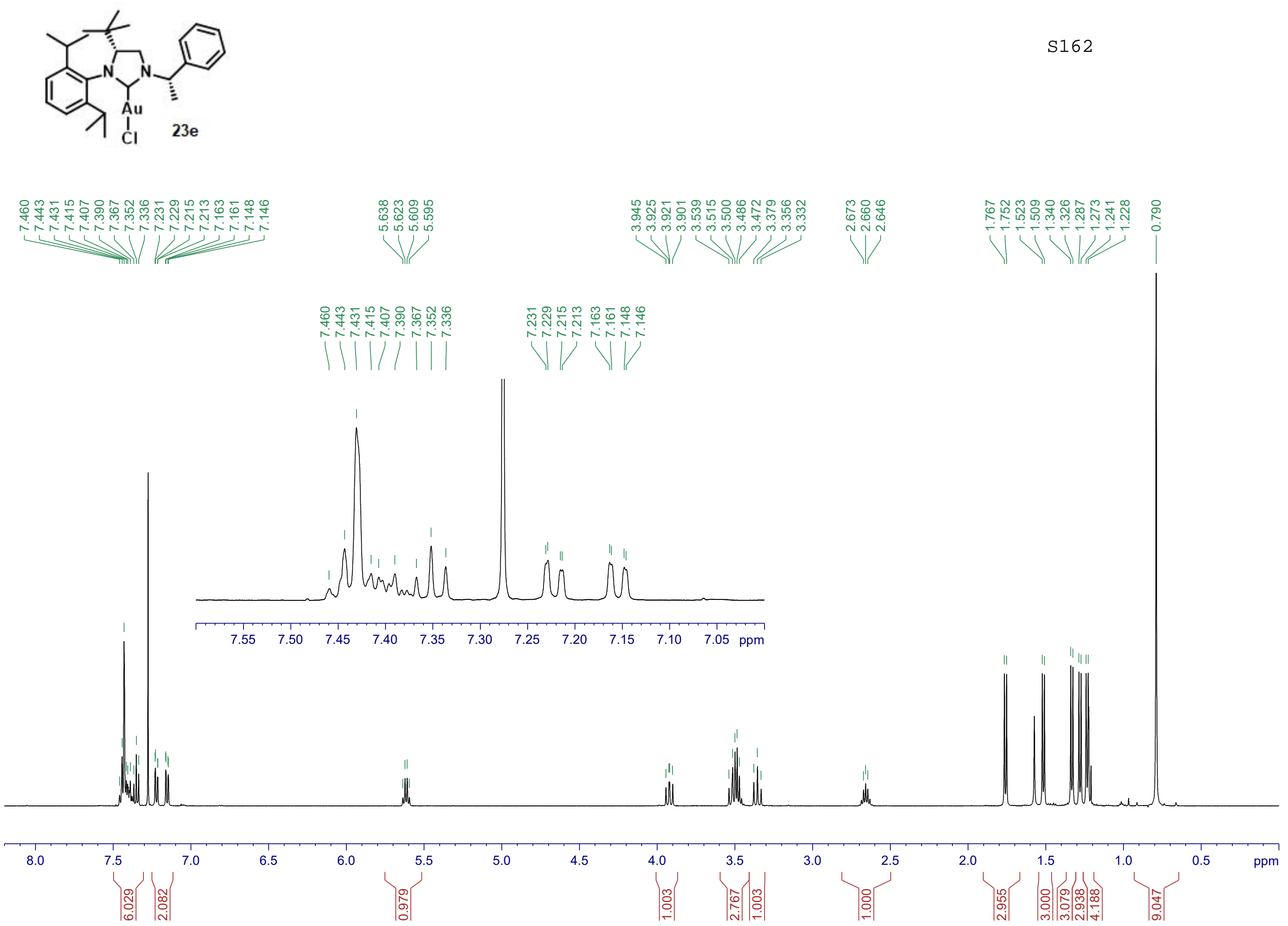

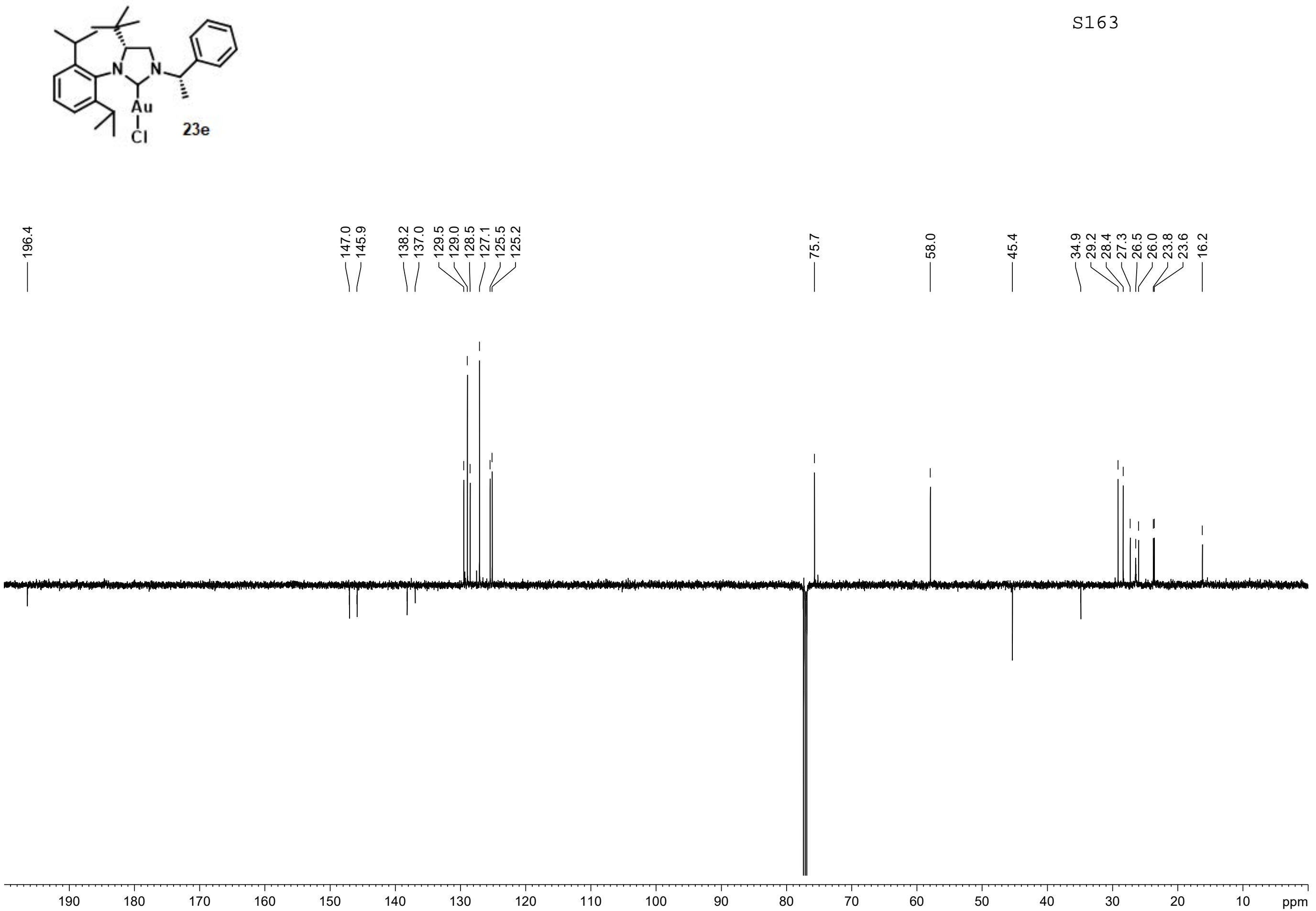

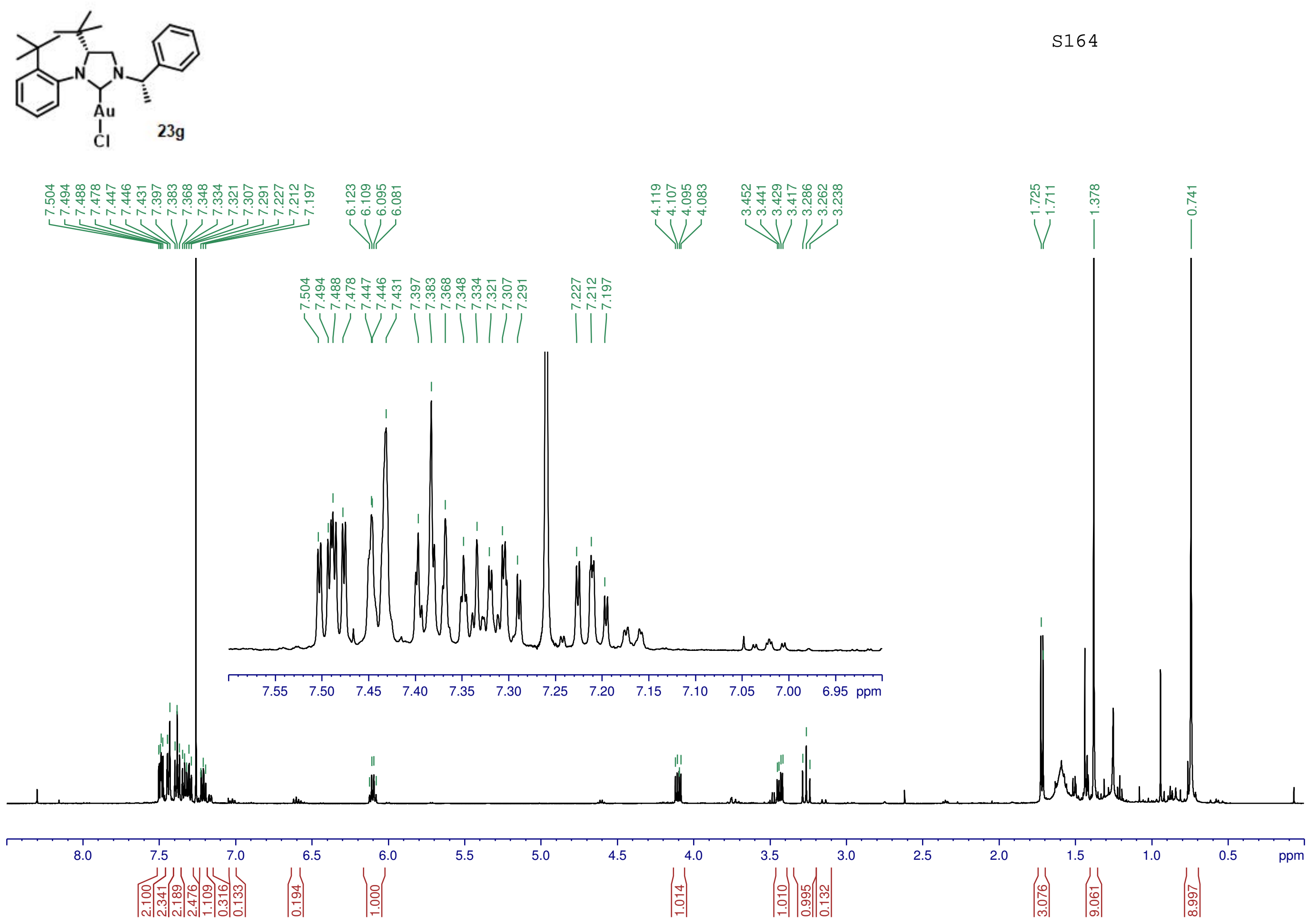

